Cochrane Database of Systematic Reviews

\title{
Compression for venous leg ulcers (Review)
}

O'Meara S, Cullum N, Nelson EA, Dumville JC

O'Meara S, Cullum N, Nelson EA, Dumville JC.

Compression for venous leg ulcers.

Cochrane Database of Systematic Reviews 2012, Issue 11. Art. No.: CD000265.

DOI: 10.1002/14651858.CD000265.pub3.

www.cochranelibrary.com 
TABLE OF CONTENTS

HEADER

ABSTRACT

PLAIN LANGUAGE SUMMARY

BACKGROUND

OBJECTIVES

METHODS

RESULTS

Figure 1.

Figure 2.

Figure 3.

Figure 4.

Figure 5.

Figure 6.

Figure 7.

Figure 8.

Figure 9.

Figure 10

DISCUSSION

AUTHORS' CONCLUSIONS

ACKNOWLEDGEMENTS

REFERENCES

CHARACTERISTICS OF STUDIES

DATA AND ANALYSES

Analysis 1.1. Comparison 1 Compression vs no compression (primary dressing only), Outcome 1 Ulcers completely healed at 6 months.

Analysis 2.1. Comparison 2 Compression vs no compression (non-compressive bandage), Outcome 1 Patients with complete healing at 1 year.

Analysis 3.1. Comparison 3 Compression vs no compression (usual treatment), Outcome 1 Patients with complete healing at 3 months.

Analysis 3.2. Comparison 3 Compression vs no compression (usual treatment), Outcome 2 Patients with complete healing at 1 year.

Analysis 3.3. Comparison 3 Compression vs no compression (usual treatment), Outcome 3 Patients with recurrence during 1 year follow-up.

Analysis 4.1. Comparison 4 Single-component compression (elastic bandage) vs single-component compression (paste bandage), Outcome 1 Patients with complete healing at 3 months.

Analysis 4.2. Comparison 4 Single-component compression (elastic bandage) vs single-component compression (paste bandage), Outcome 2 Percentage change during trial relative to baseline ulcer area.

Analysis 4.3. Comparison 4 Single-component compression (elastic bandage) vs single-component compression (paste bandage), Outcome 3 Healing rate (cm squared per week adjusted for baseline ulcer perimeter).

Analysis 5.1. Comparison 5 Single-component compression vs multi-component compression (excluding paste bandages), Outcome 1 Complete healing during the trial period.

Analysis 5.2. Comparison 5 Single-component compression vs multi-component compression (excluding paste bandages), Outcome 2 Percentage change during trial relative to baseline ulcer area.

Analysis 5.3. Comparison 5 Single-component compression vs multi-component compression (excluding paste bandages), Outcome 3 Complete healing during the trial period (participants with simple VLU only).

Analysis 6.1. Comparison 6 Single-component compression vs multi-component compression (paste bandage), Outcome 1 Complete healing during the trial period.

Analysis 7.1. Comparison 7 Two-component (outer elastic) vs two-component (outer inelastic), Outcome 1 Patients with complete healing at 3-6 months.

Analysis 7.2. Comparison 7 Two-component (outer elastic) vs two-component (outer inelastic), Outcome 2 Patients with complete healing at 1 month.

Analysis 7.3. Comparison 7 Two-component (outer elastic) vs two-component (outer inelastic), Outcome 3 Patients with complete healing at 1 year. 
Analysis 8.1. Comparison 8 Two-component system vs four-layer bandage (4LB), Outcome 1 Patients with complete healing at 1 month.

Analysis 8.2. Comparison 8 Two-component system vs four-layer bandage (4LB), Outcome 2 Patients with complete healing at 3 months.

Analysis 8.3. Comparison 8 Two-component system vs four-layer bandage (4LB), Outcome 3 Patients with complete healing at 6 months up to point of withdrawal from randomised treatment.

Analysis 8.4. Comparison 8 Two-component system vs four-layer bandage (4LB), Outcome 4 Patients with complete healing at 6 months including withdrawals from randomised treatment.

Analysis 9.1. Comparison 93 components including elastic bandage vs 3 components including inelastic bandage, Outcome 1 Patients/limbs with complete healing during trial.

Analysis 10.1. Comparison 103 components including paste bandage vs 3 components including inelastic (short-stretch) bandage, Outcome 1 Limbs with complete healing at 3 months.

Analysis 11.1. Comparison 11 Charing Cross 4LB vs other 4LB, Outcome 1 Patients/limbs with complete healing during trial. ... Analysis 12.1. Comparison 12 LLB vs multi-layer short-stretch bandage (SSB), Outcome 1 Patients with complete healing during trial period based on IPD.

Analysis 12.2. Comparison 12 LLB vs multi-layer short-stretch bandage (SSB), Outcome 2 Limbs completely healed at 3 months (Duby 1993, IPD unavailable).

Analysis 12.3. Comparison 12 4LB vs multi-layer short-stretch bandage (SSB), Outcome 3 Hazard ratio estimates for time to healing based on IPD (fixed-effect).

Analysis 12.4. Comparison 12 LLB vs multi-layer short-stretch bandage (SSB), Outcome 4 Hazard ratio estimates for time to healing based on IPD (random-effects).

Analysis 12.5. Comparison 12 LLB vs multi-layer short-stretch bandage (SSB), Outcome 5 Hazard ratio estimates for time to healing based on IPD (fixed-effect, Partsch 2001 removed).

Analysis 12.6. Comparison 12 LLB vs multi-layer short-stretch bandage (SSB), Outcome 6 Hazard ratio estimates for time to healing based on IPD (random-effects, Partsch 2001 removed).

Analysis 12.7. Comparison 12 LLB vs multi-layer short-stretch bandage (SSB), Outcome 7 Incidence of any type of adverse event based on IPD (fixed-effect).

Analysis 12.8. Comparison 12 LLB vs multi-layer short-stretch bandage (SSB), Outcome 8 Incidence of any type of adverse event based on IPD (random effects).

Analysis 12.9. Comparison 12 LLB vs multi-layer short-stretch bandage (SSB), Outcome 9 Incidence of bandage-related adverse events based on IPD.

Analysis 12.10. Comparison 12 4LB vs multi-layer short-stretch bandage (SSB), Outcome 10 Number of adverse events (any type) based on IPD (fixed-effect).

Analysis 12.11. Comparison 12 4LB vs multi-layer short-stretch bandage (SSB), Outcome 11 Number of adverse events (any type) based on IPD (random-effects).

Analysis 12.12. Comparison 12 LLB vs multi-layer short-stretch bandage (SSB), Outcome 12 Number of adverse events (bandage-related) based on IPD.

Analysis 13.1. Comparison 13 Four-layer bandage (4LB) vs compression system with paste bandage as the base, Outcome 1 Patients/limbs with complete healing during trial.

Analysis 13.2. Comparison 13 Four-layer bandage (4LB) vs compression system with paste bandage as the base, Outcome 2 Patients/limbs with complete healing at 3 months (random-effects).

Analysis 13.3. Comparison 13 Four-layer bandage (4LB) vs compression system with paste bandage as the base, Outcome 3 Percentage reduction of baseline ulcer area at 6 months.

Analysis 13.4. Comparison 13 Four-layer bandage (4LB) vs compression system with paste bandage as the base, Outcome 4 Healing rate (pooled).

Analysis 14.1. Comparison 14 Adjustable inelastic compression boot vs other compression system, Outcome 1 Healing rate. ... Analysis 15.1. Comparison 15 Single-layer compression stocking vs paste bandage system, Outcome 1 Complete healing in trial period (varying lengths).

Analysis 15.2. Comparison 15 Single-layer compression stocking vs paste bandage system, Outcome 2 Healing rate (cm2 per week).

Analysis 16.1. Comparison 16 Low-compression stocking vs short-stretch bandage (SSB), Outcome 1 Patients with complete healing at 3 months.

Analysis 16.2. Comparison 16 Low-compression stocking vs short-stretch bandage (SSB), Outcome 2 Patients with complete healing at 6 months.

Analysis 17.1. Comparison 17 High-compression stockings vs short-stretch bandage (SSB), Outcome 1 Patients with complete healing at 2-4 months (fixed-effect). 
Analysis 17.2. Comparison 17 High-compression stockings vs short-stretch bandage (SSB), Outcome 2 Patients with complete healing at 2-4 months (random-effects).

Analysis 17.3. Comparison 17 High-compression stockings vs short-stretch bandage (SSB), Outcome 3 Percentage reduction of baseline ulcer area at 3 months.

Analysis 18.1. Comparison 18 Compression stocking vs two-component bandage system, Outcome 1 Patients with complete healing at 3 months.

Analysis 19.1. Comparison 19 Compression stocking vs 4LB, Outcome 1 Patients with complete healing at 3 months. .............

Analysis 20.1. Comparison 20 Tubular compression vs short-stretch bandage (SSB), Outcome 1 Patients with complete healing at 3 months.

Analysis 21.1. Comparison 21 Tubular compression vs tubular plus 1 elastic bandage, Outcome 1 Patients with complete healing at six months.

Analysis 22.1. Comparison 22 Tubular compression vs tubular plus 2 elastic bandages, Outcome 1 Patients with complete healing at six months.

Analysis 23.1. Comparison 23 Tubular plus 1 elastic bandage vs tubular plus 2 elastic bandages, Outcome 1 Patients with complete healing at six months.

ADDITIONAL TABLES

APPENDICES

WHAT'S NEW

HISTORY

CONTRIBUTIONS OF AUTHORS 
[Intervention Review]

\section{Compression for venous leg ulcers}

Susan O'Meara ${ }^{1}$, Nicky Cullum² ${ }^{2}$ E Andrea Nelson ${ }^{3}$, Jo C Dumville ${ }^{1}$

1Department of Health Sciences, University of York, York, UK. 2School of Nursing, Midwifery and Social Work, University of Manchester, Manchester, UK. ${ }^{3}$ School of Healthcare, University of Leeds, Leeds, UK

Contact address: Susan O'Meara, Department of Health Sciences, University of York, Area 3 Seebohm Rowntree Building, Heslington, York, YO10 5DD, UK.smo4@york.ac.uk.

Editorial group: Cochrane Wounds Group

Publication status and date: Edited (no change to conclusions), published in Issue 3, 2013.

Citation: O'Meara S, Cullum N, Nelson EA, Dumville JC. Compression for venous leg ulcers. Cochrane Database of Systematic Reviews 2012, Issue 11. Art. No.: CD000265. DOI: 10.1002/14651858.CD000265.pub3.

Copyright @ 2013 The Cochrane Collaboration. Published by John Wiley \& Sons, Ltd.

\section{A B S T R A C T}

\section{Background}

Up to one percent of people in industrialised countries will suffer from a leg ulcer at some time. The majority of these leg ulcers are due to problems in the veins, resulting in an accumulation of blood in the legs. Leg ulcers arising from venous problems are called venous (or varicose or stasis) ulcers. The main treatment is the application of a firm compression garment (bandage or stocking) in order to aid venous return. There is a large number of compression garments available and it was unclear whether they are effective in treating venous ulcers and, if so, which method of compression is the most effective.

\section{Objectives}

To undertake a systematic review of all randomised controlled trials (RCTs) evaluating the effects on venous ulcer healing of compression bandages and stockings.

Specific questions addressed by the review are:

1. Does the application of compression bandages or stockings aid venous ulcer healing?

2. Which compression bandage or stocking system is the most effective?

\section{Search methods}

For this second update we searched: the Cochrane Wounds Group Specialised Register (31 May 2012); the Cochrane Central Register of Controlled Trials (CENTRAL) (The Cochrane Library Issue 5, 2012); Ovid MEDLINE (1950 to May Week 4 2012); Ovid MEDLINE (In-Process \& Other Non-Indexed Citations 30 May 2012); Ovid EMBASE (1980 to 2012 Week 21); and EBSCO CINAHL (1982 to 30 May 2012). No date or language restrictions were applied.

\section{Selection criteria}

RCTs recruiting people with venous leg ulceration that evaluated any type of compression bandage system or compression stockings were eligible for inclusion. Eligible comparators included no compression (e.g. primary dressing alone, non-compressive bandage) or an alternative type of compression. RCTs had to report an objective measure of ulcer healing in order to be included (primary outcome for the review). Secondary outcomes of the review included ulcer recurrence, costs, quality of life, pain, adverse events and withdrawals. There was no restriction on date, language or publication status of RCTs.

\section{Data collection and analysis}

Details of eligible studies were extracted and summarised using a data extraction table. Data extraction was performed by one review author and verified independently by a second review author. 


\section{Main results}

Forty-eight RCTs reporting 59 comparisons were included (4321 participants in total). Most RCTs were small, and most were at unclear or high risk of bias. Duration of follow-up varied across RCTs. Risk ratio (RR) and other estimates are shown below where RCTs were pooled; otherwise findings refer to a single RCT.

There was evidence from eight RCTs (unpooled) that healing outcomes (including time to healing) are better when patients receive compression compared with no compression.

Single-component compression bandage systems are less effective than multi-component compression for complete healing at six months (one large RCT).

A two-component system containing an elastic bandage healed more ulcers at one year than one without an elastic component (one small $\mathrm{RCT})$.

Three-component systems containing an elastic component healed more ulcers than those without elastic at three to four months (two RCTs pooled), RR 1.83 (95\% Cl 1.26 to 2.67 ), but another RCT showed no difference between groups at six months.

An individual patient data meta-analysis of five RCTs suggested significantly faster healing with the four-layer bandage (4LB) than the short stretch bandage (SSB): median days to healing estimated at 90 and 99 respectively; hazard ratio $1.31(95 \% \mathrm{Cl} 1.09$ to 1.58$)$.

High-compression stockings are associated with better healing outcomes than SSB at two to four months: RR $1.62(95 \% \mathrm{Cl} 1.26$ to 2.10$)$, estimate from four pooled RCTs.

One RCT suggested better healing outcomes at 16 months with the addition of a tubular device plus single elastic bandage to a base system of gauze and crepe bandages when compared with two added elastic bandages. Another RCT had three arms; when one or two elastic bandages were added to a base three-component system that included an outer tubular layer, healing outcomes were better at six months for the two groups receiving elastic bandages.

There is currently no evidence of a statistically significant difference for the following comparisons:

- alternative single-component compression bandages (two RCTs, unpooled);

- two-component bandages compared with the 4LB at three months (three RCTs pooled);

- alternat ive versions of the 4LB for complete healing at times up to and including six months (three RCTs, unpooled);

- $4 \mathrm{LB}$ compared with paste bandage for complete healing at three months (two RCTs, pooled), six months or one year (one RCT for each time point);

- adjustable compression boots compared with paste bandages for the outcome of change in ulcer area at three months (one small RCT);

- adjustable compression boots compared with the 4LB with respect to complete healing at three months (one small RCT);

- single-layer compression stocking compared with paste bandages for outcome of complete healing at four months (one small RCT) and 18 months (another small RCT);

- low compression stocking compared with SSB for complete healing at three and six months (one small RCT);

- compression stockings compared with a two-component bandage system and the 4LB for the outcome of complete healing at three months (one small, three-armed RCT); and,

- tubular compression compared with SSB (one small RCT) for complete healing at three months.

Secondary outcomes: 4LB was more cost-effective than SSB. It was not possible to draw firm conclusions regarding other secondary outcomes including recurrence, adverse events and health-related quality of life.

\section{Authors' conclusions}

Compression increases ulcer healing rates compared with no compression. Multi-component systems are more effective than singlecomponent systems. Multi-component systems containing an elastic bandage appear to be more effective than those composed mainly of inelastic constituents. Two-component bandage systems appear to perform as well as the 4LB. Patients receiving the 4LB heal faster than those allocated the SSB. More patients heal on high-compression stocking systems than with the SSB. Further data are required before the difference between high-compression stockings and the 4LB can be established.

\section{PLAIN LANGUAGE SUMMARY}

\section{Compression bandages and stockings to help the healing of venous leg ulcers}

Venous leg ulcers can occur when blood returning from veins in the legs to the heart is slow or obstructed. These ulcers can take a long time to heal (weeks or months) and can cause distress to patients, as well as being very costly to the health service. Compression bandages help blood to return to the heart from the legs, and there are a variety of types of bandage systems available; some are just a single bandage, while others require the application of several different types of bandages to the leg. Compression stockings are sometimes used 
as an alternative to compression bandages. This review examined the effectiveness of compression bandages versus no compression, and compared different types of compression bandages and stockings. We looked at how well these different treatments work in terms of ulcer healing. We found that applying compression was better than not using compression, and that multi-component bandages worked better than single-component systems. Multi-component systems (bandages or stockings) appear to perform better when one part is an elastic (stretchy) bandage. A very detailed analysis showed that a system called the 'four-layer bandage' or '4LB' (i.e. four different bandages applied to the leg, including an elastic one) heals ulcers faster than the 'short-stretch bandage' or 'SSB' (a type of bandage with very minimal stretch). 


\section{B A C K G R O U N D}

\section{Venous leg ulcers: the extent of the problem and management with compression}

Leg ulceration is typically a chronic recurring condition with duration of episodes of ulceration ranging from a matter of weeks to more than 10 years (Callam 1985; Moffatt 1995; Noonan 1998; Lorimer 2003; Moffatt 2004; Vowden 2009b).

A systematic review of the epidemiological literature from developed countries reported prevalence rates for any aetiology of open lower limb ulceration ranging from 1.2 to 11.0 per 1000 population (cases validated) (Graham 2003). Recent surveys undertaken in the UK collected data from populations in Wandsworth, London (Moffatt 2004), Hull and East Yorkshire (Srinivasaiah 2007), and Bradford and Airedale primary care trust (Vowden 2009a). The prevalence of venous leg ulceration was estimated as 0.23 per 1000 population in London (Moffatt 2004), 0.44 per 1000 in Hull and East Yorkshire (Srinivasaiah 2007), and 0.39 per 1000 in Bradford (Vowden 2009a; Vowden 2009b). The lower estimates in the recent UK surveys relative to the earlier worldwide literature (Graham 2003) - searches done during 2000 - might be explained by improvements in treatment as well as the broad versus narrow selection criteria for leg ulcers (Vowden 2009b). The epidemiological data have consistently suggested that prevalence increases with age and is higher among women (Callam 1992a; Graham 2003; Lorimer 2003; Moffatt 2004; Vowden 2009b).

Leg ulcers are associated with considerable cost to patients and to healthcare providers. Two systematic reviews summarised the literature on health-related quality of life in patients with leg ulcers; one included 24 studies (Herber 2007), and the other 37 (Persoon 2004). Both reviews included qualitative and quantitative evaluations, and reported that presence of leg ulceration was associated with pain, restriction of work and leisure activities, impaired mobility and social isolation.

The estimated annual cost of leg ulcer treatment to the National Health Service (NHS) in the UK was between GBP 230 million and GBP 400 million during 1990-1991 (Bosanquet 1992). A later study estimated the cost of leg ulcer care within individual UK district health authorities at GBP 212,700 to GBP 333,377 annually per district (price year 1999) (Ellison 2002). Ragnarson Tennvall 2005 estimated that the average cost of treating a venous leg ulcer in the UK was between EUR 814 and EUR 1994 (price year 2002), with higher costs associated with larger and more chronic wounds. Drew et al estimated that GBP 3.21 million was spent on dressings and other materials in Hull and East Yorkshire during 2005-2006; the cost of nursing time for wound care during the same period was GBP 6.08 million (Drew 2007). Vowden et al estimated that in Bradford, GBP 1.69 million was spent on dressings and compression bandages and GBP 3.08 million on nursing time (2006-2007 prices) (Vowden 2009c). The latter two reports relate to the care of all types of wounds, not just venous leg ulcers. Posnett 2007 estimated the costs of venous ulcers to the UK NHS at GBP 168 million to GBP 198 million annually (price year 2005-2006). The differences between the 1990-1991 estimates and those from 2005-2006 may be explained by limitations of the source data (Posnett 2007), or a true reduction in costs attributable to lower prevalence.

Most leg ulcers are associated with venous disease. A history of deep vein thrombosis is widely regarded as a predisposing factor to venous insufficiency and, hence, venous ulceration, however, the aetiology of leg ulceration remains poorly understood. Venous insufficiency has been shown to be associated with increased hydrostatic pressure in the veins of the leg, and it is in an attempt to reverse this and aid venous return that external compression, in various forms, is applied as a therapy for venous leg ulcers (Moffatt 2007).

Various forms of bandaging have been applied over the years. In the 17th Century, compression was applied as rigid lace-up stockings, and elasticated bandages were first produced in the middle of the 19th Century (Thomas 1995). At the beginning of the 21st century wide variation remains in the management of venous leg ulcers within local areas (Srinivasaiah 2007; Vowden 2009b), and across countries (Cullen 2009). In North America, Unna's boot is a popular choice of device (Meyer 2003). This is a type of paste bandage, often impregnated with preparations such as glycerin, zinc oxide and calamine lotion (Rubin 1990; BNF 2012). Such devices can be completely inelastic or may have a degree of pliability (Moffatt 2007). In the UK the four-layer bandage (4LB) - which includes elastic components - is widely used (Meyer 2003), whilst in mainland Europe and Australia the short-stretch bandage (SSB) is standard practice (Weller 2010). This review summarises the evidence for the effectiveness of the different forms of compression bandaging and compression stockings for venous leg ulcers. Devices that apply intermittent or pulsed compression to the limb were specifically excluded from this review and have been assessed in a separate Cochrane review (Nelson 2011).

\section{Classification of different types of compression}

There are many ways of applying compression, including single components (i.e. one type of bandage or stocking) and systems consisting of multiple components (different types of bandages and stockings used together or separately). The interpretation of comparisons between compression systems has been hindered by the lack of internationally agreed performance standards, for example the classification systems for compression stockings differ between the UK and Europe. In the UK, performance indicators for bandages and compression stockings have been developed (British Standards Institute1995). Bandages are categorised as retention, support or compression, depending on their performance in standardised laboratory tests. Compression bandages are further sub-divided according to the amount of force required to extend them, and, therefore, the level of compression that they can apply to a limb. Furthermore, the laboratory performance of a bandage may not reflect its performance in clinical use, as this might depend upon operator training and application technique (specifically whether applied as a spiral or figure-of-eight, the numbers of layers applied and the amount of extension used). Compression systems commonly used for venous leg ulcers are listed below (from Thomas 1995).

\section{Classification of bandages:}

- Class 1: retention bandages. Used to retain dressings.

- Class 2: support bandages. Used to support strains and sprains, e.g. crepe. Other bandages in this category can apply mild to moderate compression, e.g. Setocrepe (Mölnlycke), when particular application techniques are used and the bandages are reapplied frequently. 
- Class 3a: light compression. These bandages exert 14 to 17 $\mathrm{mmHg}$ at the ankle when applied in a simple spiral, e.g. Elset (Mölnlycke).

- Class 3b: moderate compression. These bandages apply 18 to $24 \mathrm{mmHg}$ at the ankle when applied as a simple spiral, e.g. Granuflex Adhesive Compression Bandage (ConvaTec).

- Class 3c: high compression. These bandages apply 25 to 35 $\mathrm{mmHg}$ at the ankle when applied as a simple spiral, e.g. Setopress (Mölnlycke), and Tensopress (Smith and Nephew).

- Class 3d: extra high compression. These bandages apply up to $60 \mathrm{mmHg}$ at the ankle when applied as a simple spiral.

Compression stockings (or hosiery) can be used to treat open ulceration and to reduce the risk of recurrence post-healing. They are classified in a similar way to bandages, according to the level of compression applied to the limb. Importantly, stockings are subject to less operator variability than bandages:

- Class 1: light support, provides 14 to $17 \mathrm{mmHg}$ at the ankle. Used to treat varicose veins.

- Class 2: medium support, provides 18 to $24 \mathrm{mmHg}$ at the ankle. Used to treat more severe varicosities, and to prevent venous leg ulcers.

- Class 3: strong support, provides 25 to $35 \mathrm{mmHg}$ at the ankle. Used to treat severe chronic hypertension and severe varicose veins, and to prevent venous leg ulcers.

\section{Recent developments in the classification of compression systems}

An international, expert consensus group debated the validity of the bandage classification described above, and recommended classification based on alternative criteria (Partsch 2008b). In particular, the group made a distinction between layers and components of compression bandage systems. Whereas previously, different compression systems had been described as 'single-layer', two-layer', 'four-layer' and so on, this report proposed that application of all bandages involves some degree of overlap and therefore it is misleading to categorise any bandage system as 'single-layer'. The group recommended that the components of compression should be described, such as orthopaedic wool, crepe bandage or cohesive elastic bandages. Other recommended classification criteria include sub-bandage pressure (measured in the medial gaiter area with the patient supine) and the elastic property of the overall compression system. In terms of sub-bandage pressure, the group proposed alternative categories to those described by the British Standards Institute (British Standards Institute1995), based on in vivo measurements. Overall, the ranges of pressure proposed by the consensus group are higher than those from the British Standards Institute. The recommendation to assess the elastic property of the compression system overall has arisen from the notion that, although individual parts of a compression bandage system may be elastic, the interaction between different components might result in a system that is inelastic. In order to assess this, a measurement called the 'static stiffness index' (SSI) has been proposed; this is defined as the difference in sub-bandage pressures measured in standing and supine positions. A pressure increase of more than $10 \mathrm{mmHg}$ when the patient moves from supine to standing has been suggested to indicate inelasticity (high stiffness), and an increase of less than $10 \mathrm{mmHg}$ corresponds to elasticity (low stiffness) (Partsch 2008b). Findings from a study of haemodynamics in 42 patients with chronic venous insufficiency treated with class II compression stockings suggested that the quotient of maximum working pressure to resting pressure (a measure of stiffness) is closely related to haemodynamic improvement, with increasing quotient representing reduced venous reflux (Häfner 2001). The relationship between any of these parameters and ability to aid healing remains unknown. Where compression bandages are used as a single component, they can still be defined as 'elastic' and 'inelastic' (Partsch 2008b). The following are examples of multicomponent bandage systems (listed for illustrative purposes only; not intended as practice recommendations):

- short stretch/inelastic systems - orthopaedic padding plus one or two rolls of SSB;

- inelastic paste systems - paste bandage plus support bandage, e.g. Setocrepe ${ }^{\circledR}$ (Mölnlycke);

- two-component bandage systems - orthopaedic padding plus elastic bandage, e.g. ProGuide ${ }^{\circledR}$ (Smith \& Nephew);

- four-component bandage systems - orthopaedic padding plus support bandage (crepe) plus class $3 a$ bandage, e.g. Elset ${ }^{\circledR}$ (Mölnlycke) plus cohesive bandage, e.g. $\operatorname{Coban}^{\circledR}(3 \mathrm{M})$.

The earliest version of this review defined different compression systems in terms of the number of layers, whereas, in line with the recommendations of the consensus group outlined above, subsequent versions refer to components. Nonetheless, where a trial treatment is the original Charing Cross four-layer bandage, or a close variant of it, we have continued to use the term 'four-layer bandage' (4LB), as this is an internationally recognised bandage system. It is more difficult to classify different compression systems in relation to sub-bandage pressures or the SSI since, in general, this information is not available from clinical trial reports. In order to gain further insights into the optimal way to classify different compression systems, we consulted experts in tissue viability at the outset of the previous update of this review, and invited them to complete a survey. The survey listed different types of compression against various classifications and respondents were asked to provide the best choice of classification in their opinion. In addition, free text comments were invited. As far as possible, the information gleaned from this exercise has been used in classifying and grouping different types of compression therapy in this review, and in aiding interpretation of findings.

\section{Risks associated with use of compression}

The use of compression to enhance venous return and aid the healing of venous ulcers is not without risk. The application of external compression has been reported to lead to pressure damage in some cases (Callam 1987; Callam 1992b; Barwell 2004). This may be due to application of excessive pressure or application of pressure in the presence of arterial insufficiency. National clinical guidelines in the UK and USA recommend that all patients presenting with a leg ulcer be screened for arterial disease using Doppler measurement of the ankle-brachial pressure index (ABPI) by suitably trained staff (Royal College of Nursing 2006; AAWC 2010; SIGN 2010). Clinically significant arterial disease is often defined using a cut-off of the ABPI of below 0.8. Patients with venous leg ulceration who have ABPI between 0.5 and 0.7 may be eligible to receive modified (reduced) compression (Moffatt 2007). As part of this review, data on baseline ABPI and adverse events related to treatment have been recorded, where available. 


\section{O B JECTIVES}

To undertake a systematic review of all randomised controlled trials (RCTs) investigating the effects of compression systems (bandages and stockings) on the healing of venous leg ulcers.

Specific questions addressed by the review, and the related treatment comparisons are outlined below.

\section{Question 1: Does the application of compression bandages or stockings aid venous ulcer healing?}

- 1.1 Compression compared with primary dressing alone.

- 1.2 Compression compared with non-compressive bandages.

- 1.3 Compression compared with usual care that did not routinely include compression.

\section{Question 2: Which compression bandage or stocking} system is the most effective in terms of ulcer healing?

\subsection{Single-component compression bandage systems}

- 2.1.1 Comparison between different single-component bandage systems.

- 2.1.2 Single-component bandage systems compared with multicomponent bandage systems.

\subsection{Two-component compression bandage systems}

- 2.2.1 Comparison between different two-component bandage systems.

- 2.2.2 Two-component bandage systems compared with the 4LB.

\subsection{Three-component compression bandage systems}

- 2.3.1 Comparison between different three-component bandage systems.

\subsection{Compression bandage systems comprising four} components including an elastic component (the '4LB')

- 2.4.1 Comparison between different versions of the 4LB.

- 2.4.2 4LB compared with multi-component systems including an inelastic bandage (the SSB).

- 2.4.3 4LB compared with bandage systems having a paste bandage as the base.

\subsection{Adjustable compression boots compared with compression bandages}

- 2.5.1 Adjustable compression boots compared with paste bandages.

- 2.5.2 Adjustable compression boots compared with the 4LB.

\subsection{Compression stockings or tubular devices compared with} compression bandage systems

- 2.6.1 Compression stockings compared with paste bandages.

- 2.6.2 Compression stockings compared with inelastic bandages (the SSB).

- 2.6.3 Compression stockings compared with multi-component bandage systems.

- 2.6.4 Tubular compression compared with inelastic bandages (the SSB).
- 2.6.5 Tubular compression compared with or added to elastic bandages.

For most comparisons, data analysis was undertaken on the basis of group level (aggregate) data extracted from each trial report. The exception to this was the comparison of 4LB and SSB (Section 2.4.2) for which patient level data were available, enabling a metaanalysis of individual patient data (IPD). Methods for this were based on those developed by the Cochrane Individual Participant Data Meta-analysis Methods Group (Stewart 1995), and were prespecified in a separate protocol from the main review (available on request). Advantages of IPD meta-analyses include the opportunity to: conduct powerful time-to-event analyses; adjust for prognostic patient-level variables; update outcome data; verify the accuracy of data; and reinstate missing data (Stewart 2002). The use of patientlevel data usually allows for a more sensitive analysis, enabling the best, unbiased, estimate of treatment effect to be obtained (Stewart 1993). The following sections refer to methods used for the main review of aggregate data. An outline of the methods used for the IPD meta-analysis is provided at the end of the main methods section.

\section{METHO DS}

\section{Criteria for considering studies for this review Types of studies}

Prospective RCTs evaluating compression bandaging or stockings in the treatment of venous ulceration were eligible for inclusion. Studies using quasi-randomisation methods to allocate treatment (e.g. alternation or odd/even case numbers) were excluded. Trials were included if: the compression therapies under investigation were the only systematic difference between study arms; and if they reported an objective measure of ulcer healing such as time to complete healing, frequency of complete healing, change in wound size or healing rate. Trials reporting only subjective assessments of improvement/deterioration of the wound were excluded. There were no restrictions on the basis of language or publication status of articles.

\section{Types of participants}

RCTs recruiting people of any age with venous leg ulceration (may also be described as stasis or varicose ulceration) in any care setting were eligible for inclusion. As the method of diagnosis of venous ulceration can vary between studies, no standardised definition was applied, but each study had to refer to the use of compression for venous rather than other types of leg ulcers e.g. arterial, mixed or vasculitic.

\section{Types of interventions}

Trials evaluating any form of compression bandage or compression stockings in patients with venous leg ulcers were eligible, including those assessing the following: single-component elastic or inelastic bandage systems; multi-component bandage systems; tubular compression devices; compression boots; and compression stockings. Comparators included no compression (e.g. primary dressing alone or non-compressive bandages), or an alternative type of compression. Since the focus of Review Question 2 was to assess the relative effectiveness of different types of compression therapy, trials comparing compression with other therapies (e.g. surgery, pharmacological treatment) were excluded. In addition, 
trials reporting the use of intermittent pneumatic compression were excluded, as this therapy is the focus of another Cochrane review (Nelson 2011).

\section{Types of outcome measures}

In order to be eligible for inclusion, trials had to report at least one primary outcome.

\section{Primary outcomes}

Objective measures of healing such as:

- Time to complete healing.

- Proportion of ulcers healed within trial period.

- Change in ulcer size (surface area or volume).

- Rate of change in ulcer size (surface area or volume).

\section{Secondary outcomes}

- Ulcer recurrence.

- Costs.

- Quality of life.

- Pain.

- Adverse events.

- Patient withdrawal.

\section{Search methods for identification of studies}

\section{Electronic searches}

Details of the search strategy for the original version of this review are available in Appendix 1.

The following electronic databases were searched, without date or language restrictions, to identify RCTs that investigated the use of bandages or stockings for the treatment of venous leg ulcers:

- Cochrane Wounds Group Specialised Register (searched 31 May 2012);

- The Cochrane Central Register of Controlled Trials (CENTRAL) (The Cochrane Library Issue 5, 2012);

- Ovid MEDLINE (1950 to May Week 4 2012);

- Ovid MEDLINE (In-Process \& Other Non-Indexed Citations 30 May 2012);

- Ovid EMBASE (1980 to 2012 Week 21);

- EBSCO CINAHL (1982 to 30 May 2012).

The following search strategy was used in the The Cochrane Central Register of Controlled Trials (CENTRAL):

\#1 MeSH descriptor Occlusive Dressings explode all trees \#2 MeSH descriptor Stockings, Compression explode all trees \#3 (compression or bandag* or stocking* or hosiery or wrapp $\left.{ }^{\star}\right): \mathrm{ti}, \mathrm{ab}, \mathrm{kw}$

\#4 (\#1 OR \#2 OR \#3)

\#5 MeSH descriptor Leg Ulcer explode all trees

\#6 (varicose NEXT ulcer ${ }^{\star}$ ) or (venous NEXT ulcer ${ }^{\star}$ ) or (leg NEXT ulcer $^{\star}$ ) or (foot NEXT ulcer ${ }^{\star}$ ) or (stasis NEXT ulcer $\left.{ }^{\star}\right)$ :ti,ab,kw \#7 (\#5 OR \#6)

\#8 (\#4 AND \#7)

The search strategies for Ovid MEDLINE, Ovid EMBASE and Ovid CINAHL can be found in Appendix 2, Appendix 3 and Appendix
4 respectively. The Ovid MEDLINE search was combined with the Cochrane Highly Sensitive Search Strategy for identifying randomised trials in MEDLINE: sensitivity- and precisionmaximizing version (2008 revision); Ovid format (Lefebvre 2011). The EMBASE and CINAHL searches were combined with the trial filters developed by the Scottish Intercollegiate Guidelines Network (SIGN) (SIGN 2012).

\section{Searching other resources}

We searched the reference lists of all new studies identified in this update to reveal any further studies that were not identified through the electronic searches. For the first version of this review, experts in wound care and pharmaceutical companies were contacted to enquire about unpublished, ongoing and recently published trials. An Advisory Panel was also established that assisted by checking our reference lists for any omissions, and informed us of any unpublished, ongoing or recently completed trials. For this update we have not contacted experts or industry representatives as part of the search.

\section{Data collection and analysis \\ Data extraction and management}

References identified from searches were entered into a bibliographic software package (ProCite). Two review authors, working independently, screened the references. If either review author considered a reference to be potentially relevant, the full report was retrieved for further scrutiny. Two review authors made independent decisions about inclusion and exclusion of studies by referring each retrieved report to the selection criteria described above. Disagreements were resolved by discussion.

Details of eligible studies were extracted and summarised using a data extraction sheet. The following data were extracted:

- country of study;

- source population;

- unit of investigation (e.g., wound, limb, patient);

- patient selection criteria;

- care setting;

- baseline variables by group, e.g. age, sex, baseline area of ulcers, duration of ulceration;

- description of the interventions and numbers of patients randomised to each intervention;

- descriptions of any co-interventions or standard care;

- follow-up period;

- primary and secondary outcomes measured;

- primary and secondary outcome data;

- withdrawals from treatment, with reasons.

Attempts were made to obtain data missing from reports by contacting the authors. Studies that had been published in duplicate were included only once and all relevant data extracted. Data extraction was performed by one review author and verified independently by a second review author. Disagreements were resolved by discussion.

\section{Assessment of risk of bias in included studies}

For this review update all included trials were individually assessed using the Cochrane Collaboration tool for assessing 
risk of bias (Higgins 2011). This tool addresses six specific domains: sequence generation; allocation concealment; blinding (outcome assessment of healing); incomplete outcome data; selective outcome reporting; and other issues (for this review, baseline comparability of groups for prognostic factors such as ulcer surface area and duration). We completed a risk of bias table for each eligible trial. RCTs were classified as being at an overall high risk of bias if they were rated as 'high risk' for any one of three key domains: allocation concealment; blinded outcome assessment of healing; and completeness of outcome data. The overall classification was rated as 'unclear' if any of the key domains was individually rated as being at 'unclear risk of bias.' RCTs were classified as being at an overall low risk of bias only if all key domains were rated as 'low risk of bias'. We have presented assessment of risk of bias using a risk of bias summary figure, which presents all of the judgements in a cross-tabulation of RCT by risk of bias domain.

\section{Data synthesis}

Included trials were grouped in the narrative synthesis according to the types of compression they compared. Within each comparison group, studies were pooled when they appeared similar in terms of methods, participant characteristics, interventions and outcomes. A test of statistical heterogeneity was generated for each pooled outcome. Significant statistical heterogeneity was defined as a chisquared $P$ value of 0.1 or less and the $I^{2}$ statistic was generated in order to estimate the percentage of the variability in estimates of effect due to heterogeneity rather than chance (Higgins 2003). It has been suggested that when the 12 estimation is greater than zero, both fixed-effect and random-effects analyses should be undertaken, and any difference in estimates noted (Sterne 2008). Where clinical, methodological and statistical heterogeneity were not apparent, similar studies were pooled using a fixed-effect model. A random-effects model was additionally applied where 12 was greater than zero in the absence of apparent clinical or methodological heterogeneity. Where pooling was not possible or appropriate, individual estimates from trials were reported in the narrative synthesis.

For dichotomous outcomes (e.g. frequency of complete healing during the trial period), risk ratio (RR) estimates with $95 \%$ confidence intervals $(\mathrm{Cl})$ were calculated for each trial individually and pooled if considered appropriate. The RR was presented in preference to the odds ratio (OR), as the latter gives an inflated impression of the size of effect when event rates are high, as is the case for most trials reporting healing of chronic wounds. In studies where trial authors excluded randomised patients from their own analyses we assumed that the ulcer did not heal in these cases (therefore they were included in the denominator but not the numerator for the RR estimate of healing). Where a trial did not specify the number of patients per group prior to withdrawal, we presented complete case data.

For continuous outcomes (e.g. percentage change in ulcer surface area, healing rate in $\mathrm{cm}^{2}$ per week), the difference in means with $95 \% \mathrm{Cl}$ was calculated for each trial individually. Where appropriate, trials were pooled using the weighted difference in means. When trials assessed the same outcome using different scales (e.g. change in ulcer area in $\mathrm{cm}^{2}$ and as a percentage) but otherwise did not appear to be methodologically, clinically or statistically heterogeneous, estimates were pooled using the standardised mean difference (SMD). Where sufficient information was available from the trial report, we presented data according to intention to treat; otherwise we presented a complete case analysis.

In terms of time-to-event outcomes, it was planned to plot (and, if appropriate, pool) estimates of hazard ratio (HR) and $95 \% \mathrm{Cl}$ as presented in the trial reports using the generic inverse variance method in RevMan version 5.1.

\section{Methods for the individual patient data meta-analysis}

The methods used were broadly similar to the above with the following additional considerations.

The primary outcome was time to healing, defined as the time from the date of randomisation to the date of healing, with healing defined as complete epithelialisation of the reference wound. Data for patients with ulcers not healing within the trial period were censored on the date of last follow-up. Secondary outcomes included time to ulcer recurrence (defined as the time interval between healing and recurrence) and adverse events.

Trialists who contributed original data were asked to provide details of any additional or unpublished trials that they knew of, that had not been identified by the main search strategy described above.

Data extraction was carried out for each RCT on the basis of information provided in published trial reports. In addition, trialists were asked to provide anonymised baseline and outcome data for each randomised patient, including those excluded from their own analyses. Baseline data included sex, age, primary or recurrent ulceration, ulcer duration, ulcer area, ulcer diameter, appearance of wound bed, ulcer infection, ankle brachial pressure index (ABPI), ankle circumference, ankle mobility, patient mobility, and history of co-morbidities such as deep vein thrombosis. Outcome variables included healing status (healed or not), date of healing, recurrence status, date of recurrence, ulcer area at follow-up points during the trial, and adverse events. In addition, the trialists were asked to provide date of randomisation, allocated treatment, date of last follow-up, and details of exclusion from analysis. The risk of bias assessment was informed by published reports plus additional information from each trialist. Data from each trial were also subject to additional systematic checks to determine completeness, duplication, consistency, feasibility, and integrity of randomisation (Stewart 1995). Queries were resolved with the relevant trialist.

The patient was the unit of analysis (Altman 1997). In cases where patients had multiple wounds included in the trial, we selected the largest for inclusion in the meta-analysis. Analyses were undertaken on an intention-to-treat basis (that is, according to randomised allocation group with inclusion of all patients as the aim). Imputation was not undertaken for missing data.

In order to provide an analysis that was congruent with others in this review, we pooled data from trials for the outcome of complete healing during the trial period. We then generated a preliminary (unadjusted) analysis of time to healing using nonstratified Kaplan-Meier survival curves for both treatment groups. The dependent variable was time to healing in days, the event was a healed ulcer, and the factor was bandage type. 
Next, we generated a Cox proportional hazards model with time to healing in days as the dependent variable, healing as the event, and bandage type as a covariate. This preliminary model did not include adjustment for baseline characteristics. The main, formal, preplanned analysis entailed a Cox proportional hazards model as described above with additional covariates of sex, age, primary or recurrent ulceration, ulcer duration, ulcer area, ulcer diameter, appearance of wound bed, ulcer infection, ankle brachial pressure index, ankle circumference, ankle mobility, patient's mobility, and history of deep vein thrombosis. Covariates found to be significant at the $5 \%$ level in univariate analyses were entered simultaneously into the model. We then used a backward elimination method to generate HR estimates of treatment effect. The model was extended to include tests of statistical interaction between type of bandage and baseline characteristics using a statistical significance threshold of $p<0.05$. To take account of any differences in healing rate between study centres, we entered centres into the model as strata. This automatically included trials as strata also as no centre was in more than one trial.

The proportional hazards assumption requires that the hazard ratio is constant over time (Altman 1991). We carried out checks to assess the proportional hazards assumption by generating survival curves for each category of a covariate found to be significant during univariate analyses using the log minus log of the hazard function on the vertical axis, and the log of time to healing in days on the horizontal axis. The event was ulcer healing. Any continuous covariates were dichotomised at the median in order to be able to generate the required curves which were examined visually to determine whether the proportional hazards assumption had been upheld. This was judged to be the case if the curves representing different categories of a covariate were approximately parallel (Elashoff 1983). In addition, each covariate was assessed analytically by fitting a time-dependent Cox model for each covariate of interest. The proportional hazards assumption was considered to be upheld if, when a given variable was entered as a time dependent covariate, it failed to make a statistically significant contribution to the model (Kalbfleisch 2002). For any covariate where the proportional hazards assumption was not met, it was planned to re-run the model entering it as a time dependent covariate.

We also carried out checks to assess the linearity of the relation between the dependent variable and continuous covariates; whether time to healing was similar during early and late accrual (Bland 1998); and adequacy of model fit with regard to the relation between the number of events and the number of covariates included in each model (Peduzzi 1996).

To generate a forest plot showing the relative contribution of each trial to the meta-analysis, we derived individual trial estimates from the IPD using Cox regression with covariate adjustment as per the final adjusted model. These HR estimates were converted into the $\log \mathrm{HR}$ and its standard error, and combined using the generic inverse variance method. This allowed assessment of statistical heterogeneity between trials, using the chi-squared test (threshold $P$ value 0.1 ) and the $I^{2}$ statistic (Higgins 2002; Higgins 2003). When 12 was greater than zero, the analysis was repeated using a randomeffects model and sources of heterogeneity were investigated using sensitivity analysis.
In terms of secondary outcomes, it was planned to repeat the analyses described above for time to healing with respect to time to ulcer recurrence. Adverse events were defined in two ways: as any adverse event or those considered by the trialists to be related to the bandage. For each of these outcomes, we assessed the effect of bandage type on the prevalence of adverse events using the OR with associated $95 \% \mathrm{Cl}$. We compared the number of adverse events per patient for the two different bandage systems using a weighted difference in means with associated $95 \% \mathrm{Cl}$. For all pooled analyses of adverse events, we defined statistical heterogeneity between individual trial estimates using the criteria described above.

Survival analyses were conducted with SPSS (version 15.0). The Kaplan-Meier plot was generated with Stata SE (version 10). Adverse event analyses and forest plots were generated using RevMan (version 5.1).

\section{RES U L T S}

\section{Description of studies}

Forty-eight RCTs reporting 59 comparisons were included in this review (4321 participants in total). Three evaluations were published as conference proceedings only (Colgan 1995; Kralj 1996; Knight 1996). Details of each included RCT have been tabulated (Characteristics of included studies). Of the 48 included RCTs, nine were identified during this review update (Zuccarelli 1997; Harley 2004; Taradaj 2007; Mariani 2008; Moffatt 2008; Taradaj 2009; Brizzio 2010; Milic 2010; Szewczyk 2010); four of these were listed as "Studies awaiting classification" in the previous version of this review (Zuccarelli 1997; Harley 2004; Taradaj 2007; Moffatt 2008).

Thirty-eight studies were excluded from this review. The reasons for exclusion were:

- participants in the study were not randomised (17 studies) (Sikes 1985; Horakova 1994; Nissinen-Paatsamala 1995; Cameron 1996; Baccaglini 1998; Marston 1999; Scriven 2000; Vowden 2001; Kucharzewski 2003; Torra i Bou 2003; Alvarez 2005; Brizzio 2006; Jull 2009; Luo 2009; Szewczyk 2009; Hjerppe 2010; Van Laere 2010);

- irrelevant comparison (9 studies) (Blair 1988; Sironi 1994; Sabolinski 1995; Robson 2004; Zamboni 2004; Smith Strom 2006; Kuznetsov 2009; Heinen 2010; Serra 2010;

- participants did not have venous leg ulcers (4 studies) (Jünger 2006; Partsch 2008a; Lee 2009; Hamel-Desnos 2010);

- treatment groups differed systematically other than in terms of compression devices used (3 studies) (Northeast 1990; Olofsson 1996; Falanga 1998);

- only available as abstract (2 studies) (Walker 1996; Russo 1999);

- commentary article (2 studies) (Fuessl 2009; Ivanovic 2011);

- and healing not reported (1 study) (Cherry 1990).

Of the 38 excluded studies, 17 were identified during this review update (Falanga 1998; Robson 2004; Alvarez 2005; Brizzio 2006; Partsch 2008a; Fuessl 2009; Jull 2009; Kuznetsov 2009; Lee 2009; Luo 2009; Szewczyk 2009; Hamel-Desnos 2010; Heinen 2010; Hjerppe 2010; Serra 2010; Van Laere 2010; Ivanovic 2011); two of these were awaiting classification previously (Alvarez 2005; and Jawien 2008, now secondary reference to Szewczyk 2009). See Characteristics of excluded studies for full details. 
One RCT previously listed as awaiting classification remains in this category (Moffatt 2003b); in addition, there are six new evaluations awaiting classification as a result of this review update (Bertaux 2010; Mosti 2010; Harrison 2011; Mosti 2011; Taradaj 2011; Wong 2012), Characteristics of studies awaiting classification.

Previously, no ongoing studies were recorded, but three were identified for this update (Dumville 2009; Weller 2010; Matos de Abreu 2011), Characteristics of ongoing studies.

Just over a third of the included RCTs (18/48 or $38 \%$ ) were conducted in the UK (Charles 1991; Callam 1992b; Travers 1992; Duby 1993; Wilkinson 1997; Gould 1998; Morrell 1998; Scriven 1998; Taylor 1998; Moffatt 1999; Moody 1999; Vowden 2000; Meyer 2002; Meyer 2003; Moffatt 2003a; Franks 2004; Iglesias 2004; Nelson 2007a). Two were performed in Ireland (Colgan 1995; O'Brien 2003), 17 in mainland Europe (Eriksson 1984; Eriksson 1986; Kralj 1996; Zuccarelli 1997; Danielsen 1998; Partsch 2001; Ukat 2003; Jünger 2004a; Jünger 2004b; Polignano 2004a; Polignano 2004b; Milic 2007; Taradaj 2007; Mariani 2008; Taradaj 2009; Milic 2010; Szewczyk 2010), one in Turkey (Koksal 2003), one in Tasmania, Australia (Harley 2004), one in Argentina (Brizzio 2010), and seven in the USA (Hendricks 1985; Kikta 1988; Rubin 1990; Cordts 1992; Knight 1996; DePalma 1999; Blecken 2005). One international trial was conducted in the UK, USA and Canada (Moffatt 2008). In terms of the type of setting, all RCTs (where described) were conducted in outpatient and community settings, with three trials recruiting some hospitalised patients as part of the sample (Kralj 1996; Ukat 2003; Polignano 2004a).

The number of patients in the included RCTs ranged from 10 to 387 . Forty percent of the included trials had sample sizes of 50 patients or fewer, and the majority of trials $(67 \%)$ recruited 100 patients or fewer. Nine trials reported an a priori sample size estimation in relation to a healing outcome (Morrell 1998; Partsch 2001; Meyer 2003; Moffatt 2003a; O'Brien 2003; Ukat 2003; Franks 2004; Iglesias 2004; Nelson 2007a). Three evaluations were designed as noninferiority trials and presented a proposed non-inferiority margin (Moffatt 1999; Jünger 2004a; Jünger 2004b). Non-inferiority trials are designed to establish whether the study (or new) treatment is not worse than the control treatment by more than a small, pre-specified amount; this amount is known as the non-inferiority margin (European Medicines Agency 2005). Two more studies included some information about the intended sample size but did not show the full details of the estimation (Milic 2007; Brizzio 2010); one failed to recruit the entire intended sample (Polignano 2004a); one included a post hoc assessment of statistical power with respect to healing (Meyer 2002); and one included an a priori estimation for a non-healing outcome (bandage slippage) (Moffatt 2008). The remaining 31 trials (65\%) did not report any information about statistical power or sample size estimation.

All patients in the included RCTs were deemed to have venous ulceration, and the majority of trials (39/48 or $81 \%)$ specified a cut-off value of ankle brachial pressure index (ABPI) to exclude clinically significant arterial disease at baseline. The cut-off point for application of compression was 0.8 in the majority of these studies (27/39 or $69 \%)$, other values being 0.7 and 0.75 in one trial each, 0.9 in nine trials and 1.0 in one trial. Most of the trial reports provided some information on patient selection criteria. Four trials presented minimal details, describing only the cut-off value for ABPI (Charles 1991; Duby 1993; Taylor 1998; Ukat 2003), and three early trials did not include any details at all relating to inclusion and exclusion of patients, apart from the stipulation of having a venous leg ulcer (Hendricks 1985; Eriksson 1986; Knight 1996).

The amount of pressure applied to a leg depends on bandage application or stocking-fitting technique. Overall, few details relating to the techniques used for applying compression or relevant staff experience and training were reported in the included RCTs. Some reports stated that compression devices were applied according to the manufacturers' instructions (Hendricks 1985; Kikta 1988; Moody 1999; Franks 2004; Jünger 2004a; Polignano 2004a; Moffatt 2008); in other RCTs this was established through contact with the trial authors (Scriven 1998; Partsch 2001; Ukat 2003; Iglesias 2004). In some evaluations, nurses with prior experience of at least one of the evaluated compression systems provided care (Callam 1992b; Scriven 1998; Taylor 1998; Vowden 2000; Meyer 2002; Meyer 2003; Jünger 2004a; Harley 2004; Nelson 2007a; Brizzio 2010); in others training was provided in the use of at least one device for the purposes of the trial (Wilkinson 1997; Morrell 1998; Moody 1999; O'Brien 2003; Iglesias 2004; Jünger 2004b; Harley 2004; Moffatt 2008).

Information on the techniques used for bandage application was seldom presented in the included RCTs, but when available included a spiral technique (Charles 1991; Callam 1992b; Zuccarelli 1997; Moody 1999; Mariani 2008; Milic 2010; Szewczyk 2010), figureof-eight application (Meyer 2002; Meyer 2003; Mariani 2008; Taradaj 2009), and Putter technique (two bandages applied in opposite directions) (Partsch 2001). In some trials, patients or their relatives were involved in the application of compression devices: in a trial of compression boots, patients adjusted the straps between clinic visits in order to help maintain the original degree of compression (DePalma 1999); in other RCTs, patients or relatives were instructed to reapply bandages between clinic visits (Eriksson 1986; Ukat 2003; Jünger 2004b), or were involved in the application of compression stockings (Hendricks 1985; Jünger 2004b; Polignano 2004b; Mariani 2008).

For Review Question 1 (does the application of compression bandages or stockings aid venous ulcer healing?), eight RCTs were identified. Specific comparisons were as follows:

1.1 Compression compared with primary dressing alone (Eriksson 1984; Kikta 1988; Taradaj 2007).

1.2 Compression compared with non-compressive bandages (Rubin 1990).

1.3 Compression compared with usual care that did not routinely include compression (Charles 1991; Taylor 1998; Morrell 1998; O'Brien 2003).

For Review Question 2 (which compression bandage or stocking system is the most clinically effective?), six comparison categories were considered, with RCTs assembled into groups and sub-groups as follows:

For category 2.1 (single-component compression bandage systems) seven RCTs were identified overall which were grouped into the following comparisons:

2.1.1 Comparison between different single-component bandage systems (Cordts 1992; Zuccarelli 1997).

2.1.2 Single-component bandage systems compared with multicomponent bandage systems (Eriksson 1986; Travers 1992; Colgan 1995; Kralj 1996; Nelson 2007a). 
For category 2.2 (two-component compression bandage systems) six RCTs were identified and grouped as follows:

2.2.1 Comparison between different two-component bandage systems (Danielsen 1998; Moody 1999).

2.2.2 Two-component bandage systems compared with the 4LB (Moffatt 2003a; Harley 2004; Moffatt 2008; Szewczyk 2010).

For category 2.3 (three-component compression bandage systems), four RCTs were identified.

2.3.1 Comparison between different three-component bandage systems (Callam 1992b; Duby 1993; Gould 1998; Meyer 2002).

For category 2.4 (four component compression bandage system including an elastic component - i.e. the 4LB) 13 RCTs were identified and were grouped as follows:

2.4.1 Comparison between different versions of the 4LB (Wilkinson 1997; Moffatt 1999; Vowden 2000).

2.4.2 4LB compared with multi-component bandage systems including an inelastic bandage (the SSB) (Duby 1993; Scriven 1998; Partsch 2001; Ukat 2003; Franks 2004; Iglesias 2004).

2.4.3 4LB compared with bandaging systems having a paste bandage as the base (Duby 1993; Colgan 1995; Knight 1996; Meyer 2003; Polignano 2004a).
For category 2.5. (adjustable compression boots compared with compression bandages), two trials were identified.

2.5.1 Adjustable compression boots compared with paste bandages (DePalma 1999).

2.5.2 Adjustable compression boots compared with the 4LB (Blecken 2005).

For category 2.6 (Compression stockings or tubular devices compared with compression bandage systems), 11 trials were identified overall and were grouped into the following comparisons:

2.6.1 Compression stockings compared with paste bandages : (Hendricks 1985; Koksal 2003).

2.6.2 Compression stockings compared with inelastic bandages (the SSB) (Jünger 2004b; Polignano 2004b; Mariani 2008; Taradaj 2009; Brizzio 2010).

2.6.3 Compression stockings compared with multi-component bandage systems (Szewczyk 2010).

2.6.4 Tubular compression compared with inelastic bandages (the SSB) (Jünger 2004a).

2.6.5 Tubular compression compared with or added to elastic bandages(Milic 2007)(Milic 2010).

Figure 1 shows all the comparisons included in the review, and the number of evaluations for each comparison. 
Figure 1. This figure shows all the comparisons included in the review, and the number of evaluations for each comparison. The green shaded areas represent comparisons between similar compression systems. The numbers shown refer to numbers of comparisons, not numbers of RCTs. Key: 4LB = four-layer bandage The abbreviations A-N at the head of each column refer to the corresponding types of compression shown at the start of each row.

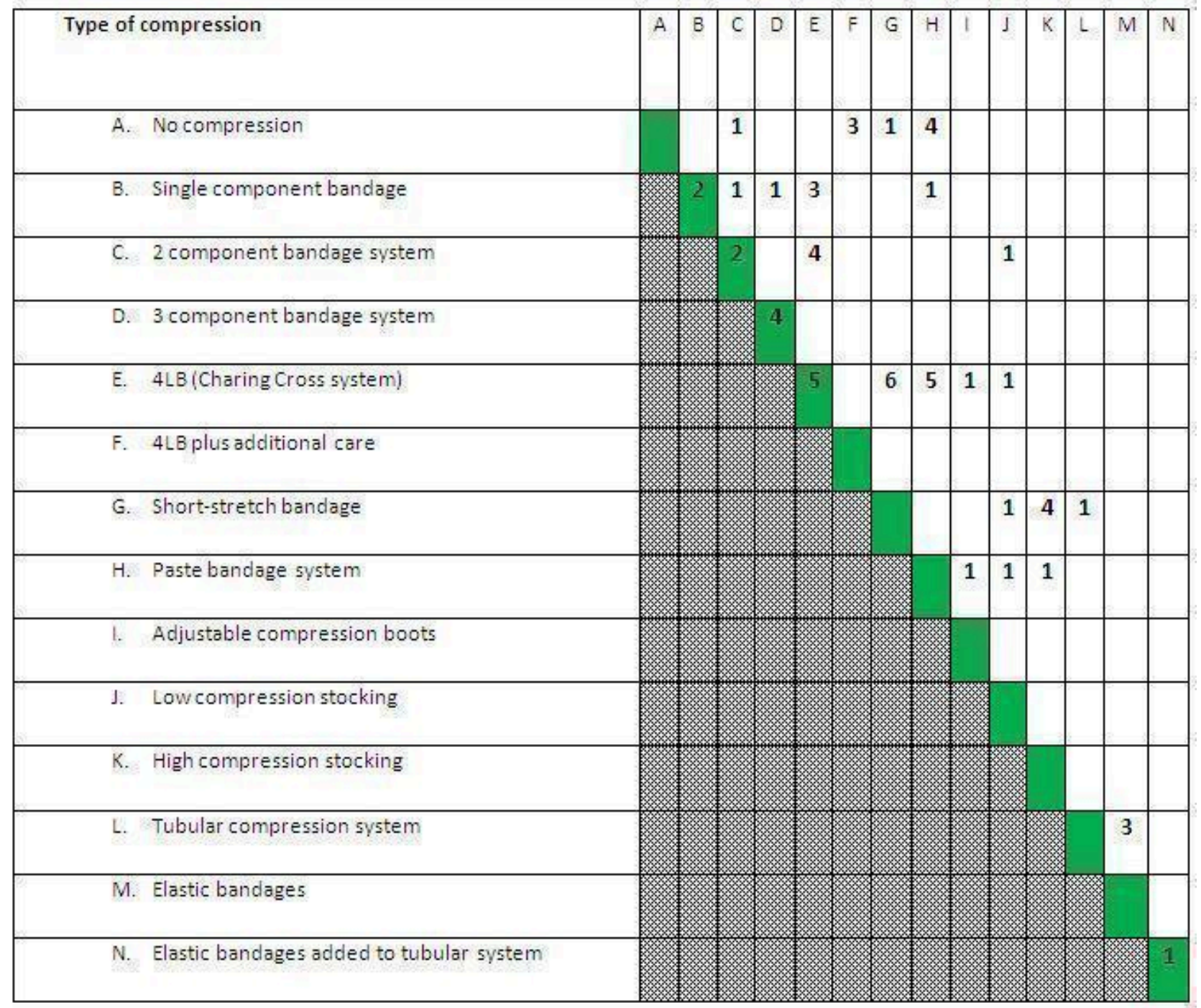

\section{Risk of bias in included studies}

The risk of bias for each included RCT has been tabulated (Characteristics of included studies). A graphical representation of this information can be found in Figure 2 and an overall summary in Figure 3. 
Figure 2. Methodological quality summary: review authors' judgements about each methodological quality item for each included study.

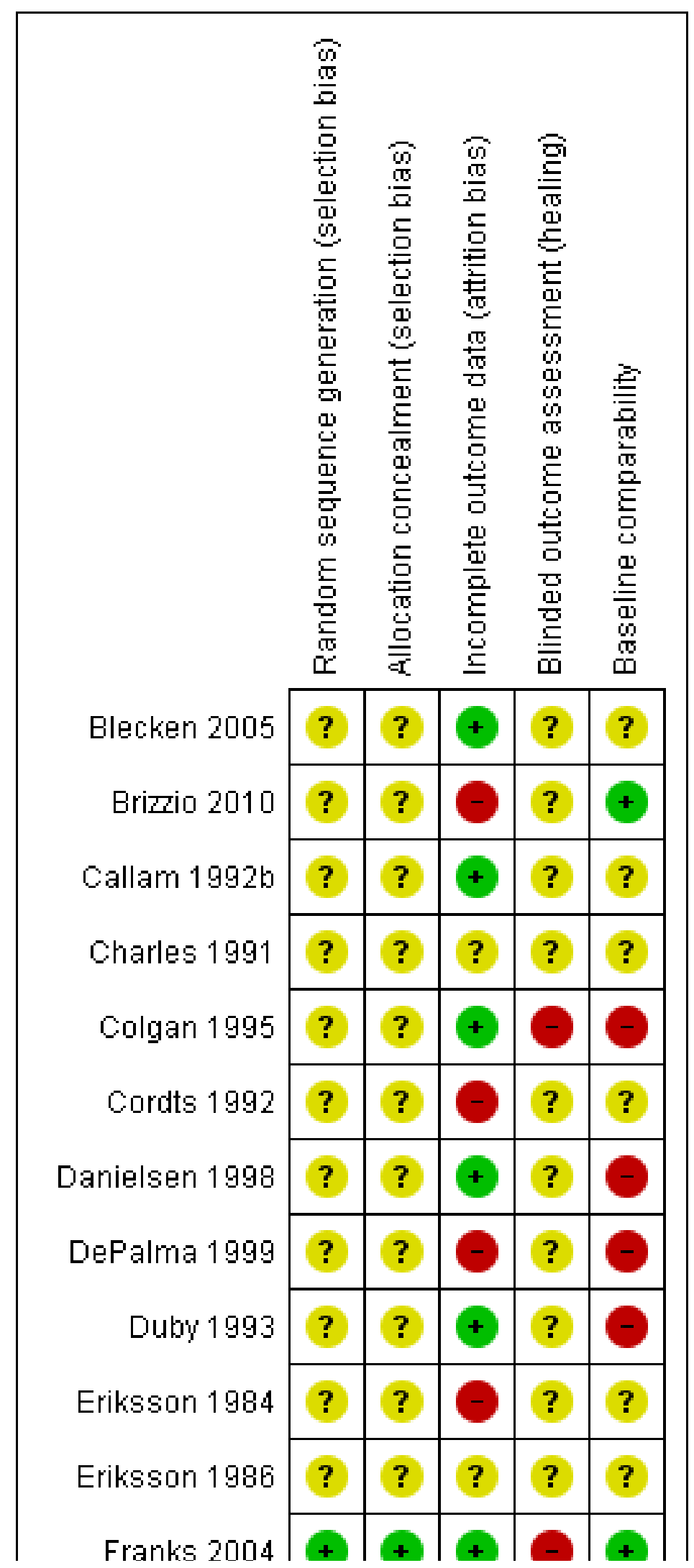


Figure 2. (Continued)

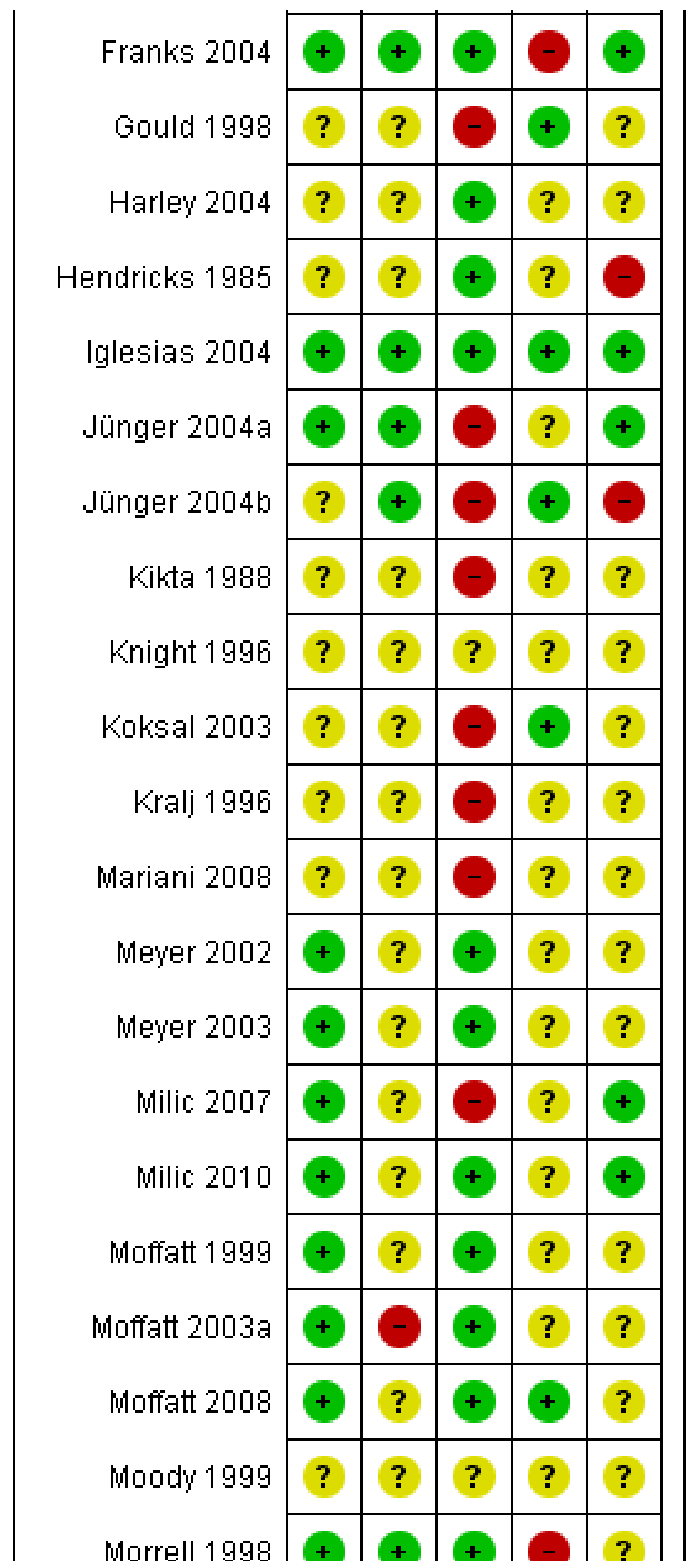


Figure 2. (Continued)

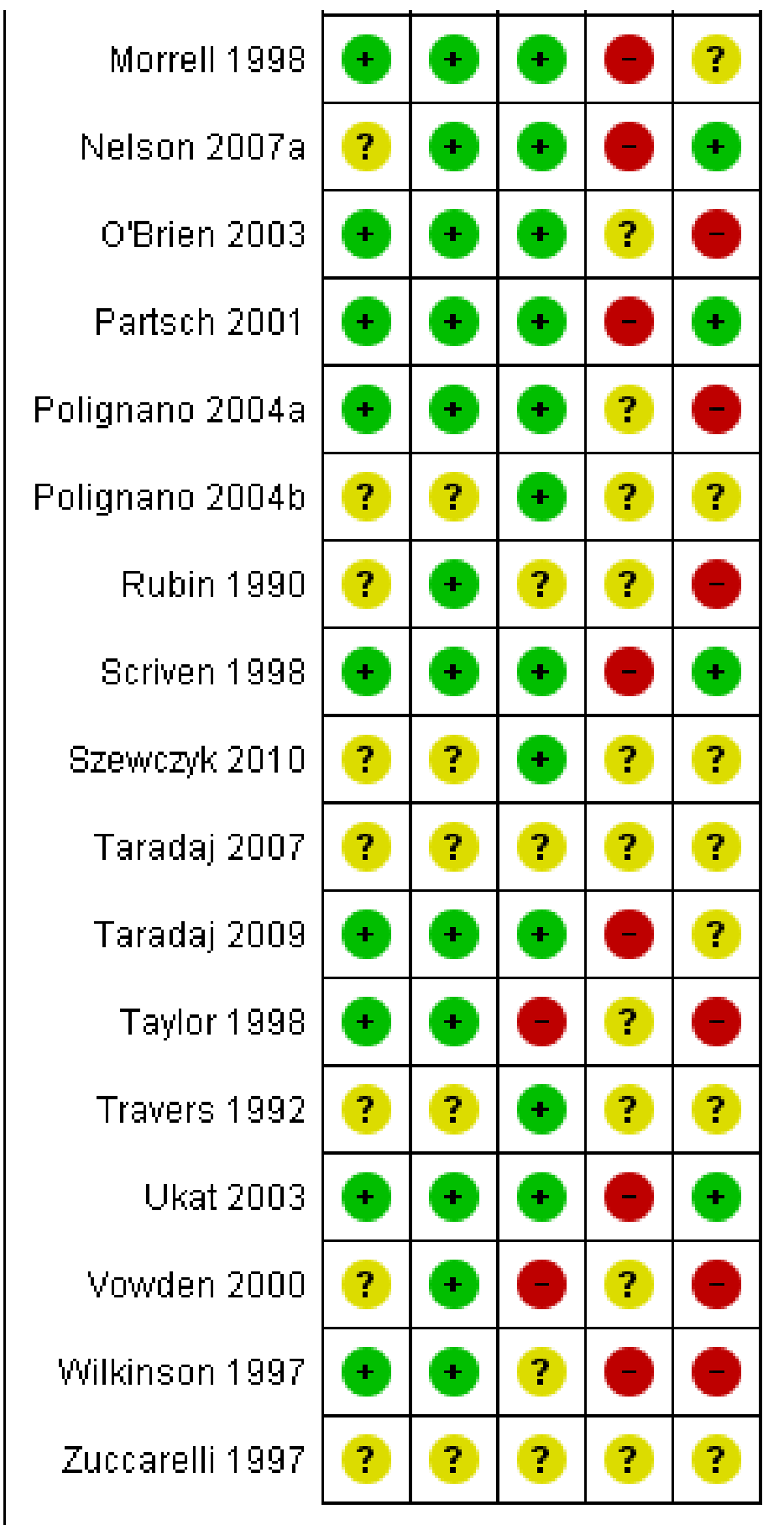


Figure 3. Methodological quality graph: review authors' judgements about each methodological quality item presented as percentages across all included studies.

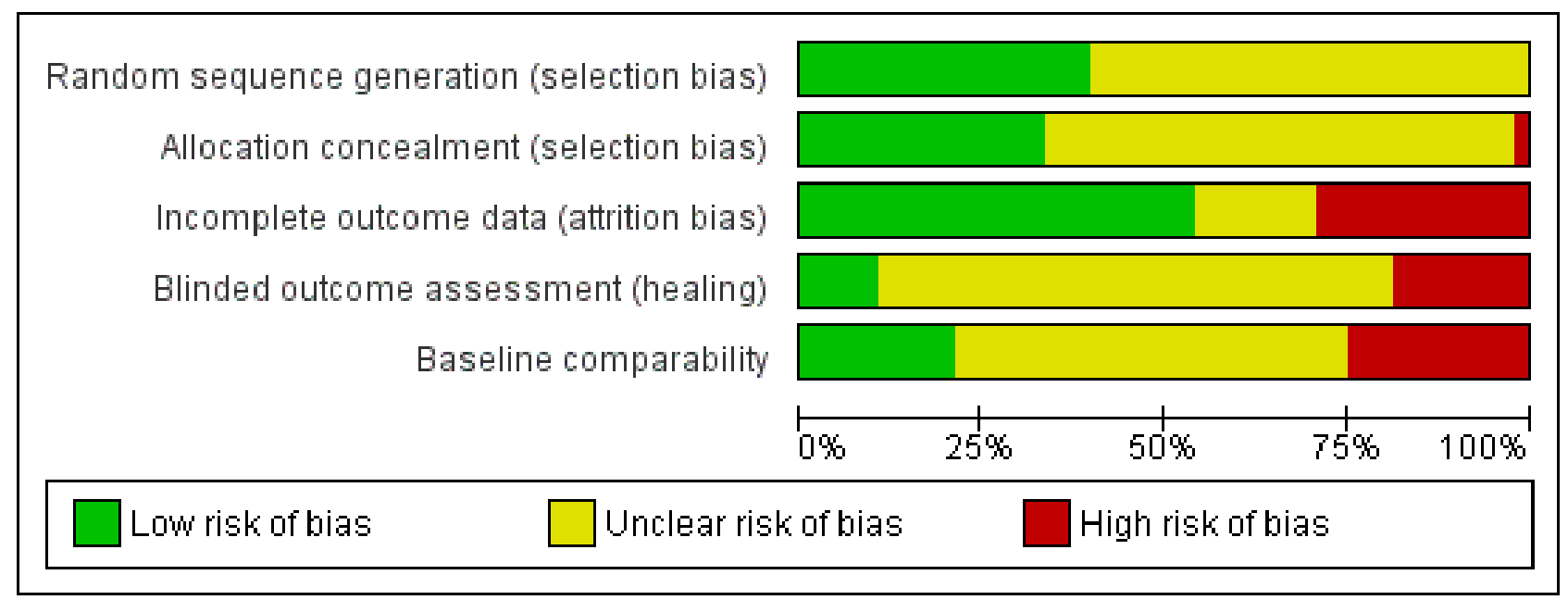

In some respects, the methodological quality of clinical trials of compression appears to be improving over time, with evaluations published within the last ten years being more likely to include an appropriate method of randomisation, with attempts to generate balanced groups at baseline, use of allocation concealment and analysis by intention to treat. Most trials do not report use of blinded outcome assessment. One RCT was classified as being at low risk of bias overall, with all risk of bias domains judged to be low risk (Iglesias 2004). Figure 4 represents a graphical presentation of risk of bias information with RCTs ordered chronologically. 
Figure 4. Methodological quality summary with trials presented in chronological order Methodological quality summary with trials presented in chronological order Risk of bias summary figure with RCTs presented in chronological order.
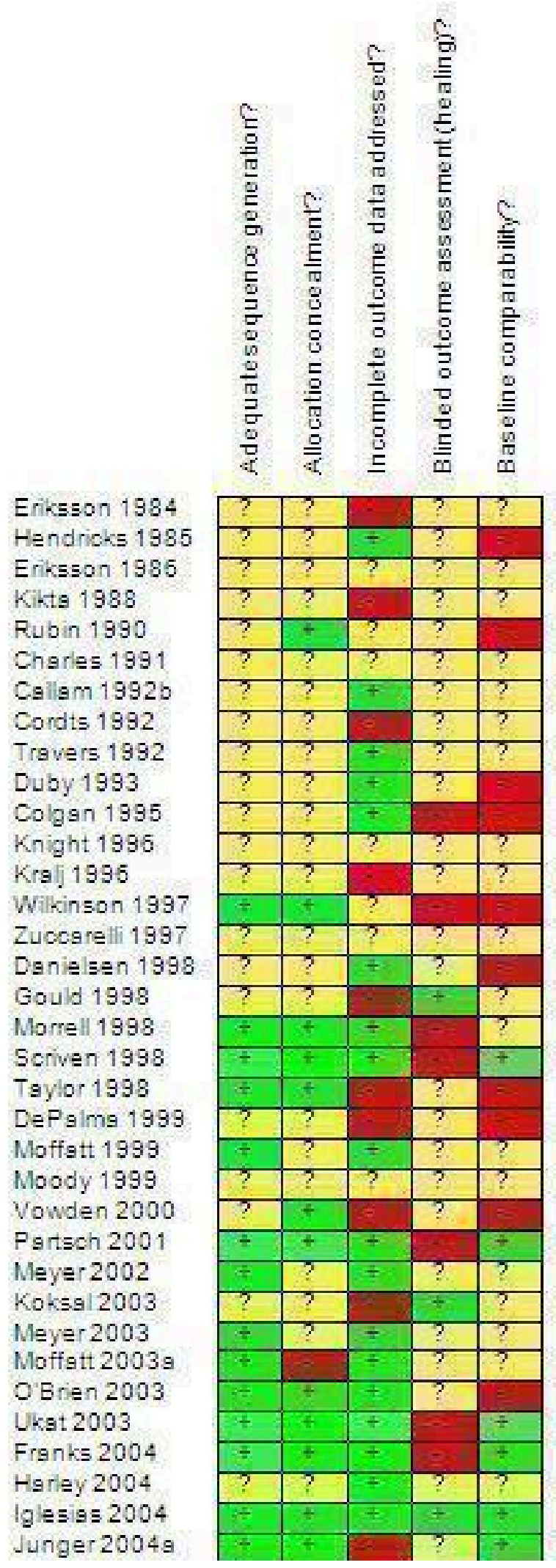
Figure 4. (Continued)

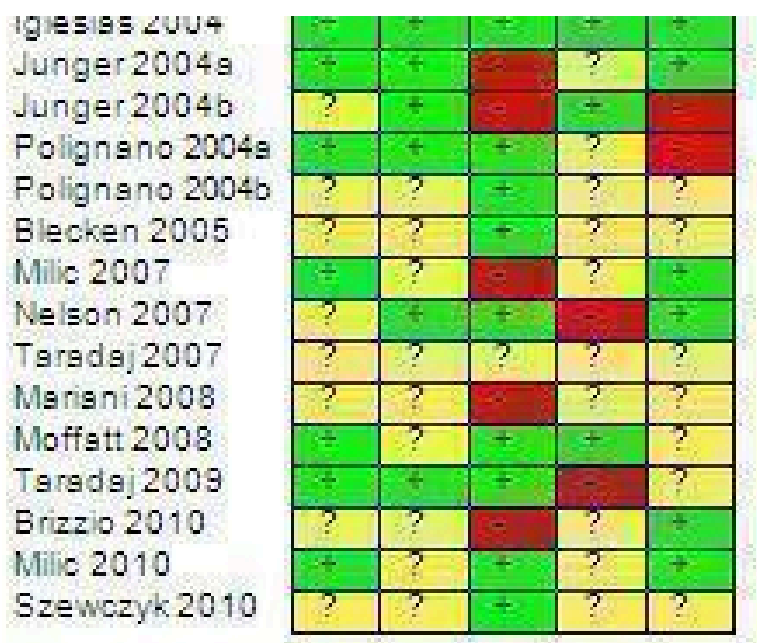

Twenty-three RCTs were classified as having an unclear risk of bias overall (Hendricks 1985; Eriksson 1986; Rubin 1990; Charles 1991; Callam 1992b; Travers 1992; Duby 1993; Knight 1996; Zuccarelli 1997; Danielsen 1998; Moffatt 1999; Moody 1999; Meyer 2002; Meyer 2003; O'Brien 2003; Harley 2004; Polignano 2004a; Polignano 2004b; Blecken 2005; Taradaj 2007; Moffatt 2008; Milic 2010; Szewczyk 2010). Six of these RCTs had all risk of bias domains rated as 'unclear', indicating poor reporting of methodological details (Eriksson 1986; Charles 1991; Knight 1996; Zuccarelli 1997; Moody 1999; Taradaj 2007). Twenty-four RCTs were classified as being at a high overall risk of bias (Eriksson 1984; Kikta 1988; Cordts 1992; Colgan 1995; Kralj 1996; Wilkinson 1997; Gould 1998; Morrell 1998; Scriven 1998; Taylor 1998; DePalma 1999; Vowden 2000; Partsch 2001; Koksal 2003; Moffatt 2003a; Ukat 2003; Franks 2004; Jünger 2004a; Jünger 2004b; Milic 2007; Nelson 2007a; Mariani 2008; Taradaj 2009; Brizzio 2010); in all cases, there was one key individual domain assessed as being at high risk of bias (usually blinded outcome assessment or incomplete outcome data). The following sections report details of risk of bias according to each domain.

\section{Allocation}

\section{Generation of the randomisation sequence}

Ten trials employed computer-generated randomisation lists (Meyer 2002; Meyer 2003; Moffatt 2003a; O'Brien 2003; Iglesias 2004; Polignano 2004a; Milic 2007; Moffatt 2008; Taradaj 2009; Milic 2010), and one used random number tables to generate the randomisation sequence (Wilkinson 1997). Other trials deemed likely to have used a satisfactory randomisation method were Morrell 1998; Scriven 1998; Taylor 1998; Moffatt 1999; Partsch 2001; Ukat 2003; Franks 2004 and Jünger 2004a. In the remaining 29 trials, treatment allocation was described as being random with no other information provided, and so they were classified as having an unclear risk of bias for this domain.

Three trials were factorial and included additional randomised comparisons of other interventions used concurrently with compression: i.e. knitted viscose dressing versus foam dressing (Callam 1992b); two different foam dressings (Franks 2004); and knitted viscose dressing versus hydrocolloid dressing and oral pentoxifylline versus placebo (Nelson 2007a). In the majority of trials, the patient was the unit of study, but in five RCTs limbs were randomised and analysed (Kikta 1988; Duby 1993; Wilkinson 1997; Scriven 1998; Blecken 2005). The methods of analysis used in these trials ignored the highly-correlated healing data from patients with both limbs included, with one exception that used withinindividual randomisation and employed an appropriate method for analysis of healing rate (Blecken 2005).

\section{Allocation concealment}

Sixteen out of 48 RCTs (33\%) were deemed to have incorporated adequate allocation concealment. These included three that used a remote telephone randomisation service (Wilkinson 1997; Iglesias 2004; Jünger 2004a), and one that used a minimisation programme which we assumed would be computerised and so include allocation concealment (Taylor 1998). In addition, 11 studies reported the use of sealed envelopes with some other detail about this method (i.e. opaque envelopes and/or opened in sequential order) and we assumed that this would amount to adequate allocation concealment (Morrell 1998; Scriven 1998; Vowden 2000; Partsch 2001; O'Brien 2003; Ukat 2003; Jünger 2004b; Polignano 2004a; Franks 2004; Nelson 2007a; Taradaj 2009). Another RCT provided sufficient information about concealment in the trial report for us to assume that procedures were satisfactory (Rubin 1990). In one evaluation, the trial authors confirmed that allocation was unconcealed (Moffatt 2003a). In the remaining 31 trials, allocation concealment was either was by sealed envelopes with no further description of the exact procedures followed (Kralj 1996), or described as "blind" randomisation with no further details (Danielsen 1998), or more commonly, not mentioned at all (Eriksson 1984; Hendricks 1985; Eriksson 1986; Kikta 1988; Charles 1991; Callam 1992b; Cordts 1992; Travers 1992; Duby 1993; Colgan 1995; Knight 1996; Zuccarelli 1997; Gould 1998; DePalma 1999; Moffatt 1999; Moody 1999; Meyer 2002; Meyer 2003; Koksal 2003; Harley 2004; Polignano 2004b; Blecken 2005; Milic 2007; Taradaj 
2007; Mariani 2008; Moffatt 2008; Brizzio 2010; Milic 2010; Szewczyk 2010). We have labelled these 31 trials as 'unclear' in terms of adequacy of allocation concealment.

\section{Blinding}

Four trials reported using blinded outcome assessment of healing (Gould 1998; Koksal 2003; Jünger 2004b; Moffatt 2008), and one incorporated blinded confirmation of healing (Iglesias 2004). For nine trials, outcome assessment was not blind (Colgan 1995; Wilkinson 1997; Morrell 1998; Scriven 1998; Partsch 2001; Ukat 2003; Franks 2004; Nelson 2007a; Taradaj 2009), and for all other studies the relevant information was either not clear or not mentioned at all.

\section{Incomplete outcome data}

Just over half of the trials (26/48 or $54 \%$ ) were classified as being at low risk of bias in terms of completeness of outcome data. Of these, 24 trials conducted analysis by intention to treat (Callam 1992b; Travers 1992; Duby 1993; Colgan 1995; Morrell 1998; Scriven 1998; Moffatt 1999; Partsch 2001; Meyer 2002; Meyer 2003; Moffatt 2003a; O'Brien 2003; Ukat 2003; Franks 2004; Harley 2004; Iglesias 2004; Polignano 2004a; Polignano 2004b; Blecken 2005; Nelson 2007a; Moffatt 2008; Taradaj 2009; Milic 2010; Szewczyk 2010). One trial presented raw data so that the review authors could analyse data according to intention to treat (Hendricks 1985). In another trial, a small number of patients were not included in the analysis because of ineligibility, the numbers being similar across treatment groups; we considered that the overall risk of bias was low (Danielsen 1998). For the remaining trials, it was either unclear whether the intentionto-treat principle had been employed, or else it was obvious that this was not the case.

\section{Other potential sources of bias}

Several prognostic studies have suggested that baseline ulcer area and duration are significant independent predictors of delayed healing of venous leg ulcers (Skene 1992; Franks 1995; Margolis 2000; Margolis 2004; Brown 2004). Therefore, each included trial was examined with reference to the balance of these variables across treatment groups. In ten trials, treatment groups appeared to be comparable at baseline (Scriven 1998; Partsch 2001; Ukat 2003; Franks 2004; Iglesias 2004; Jünger 2004a; Nelson 2007a; Milic 2007; Brizzio 2010; Milic 2010). Overall, 26 evaluations were rated as 'unclear' for this criterion for the following reasons: no data or very limited information provided (nine trials) (Eriksson 1984; Eriksson 1986; Kikta 1988; Knight 1996; Gould 1998; Moody 1999; Meyer 2002; Harley 2004; Polignano 2004b); mean rather than median values presented (medians are preferable since baseline ulcer area and duration data are usually positively skewed) (12 trials) (Charles 1991; Callam 1992b; Cordts 1992; Travers 1992; Kralj 1996; Zuccarelli 1997; Morrell 1998; Koksal 2003; Blecken 2005; Taradaj 2007; Moffatt 2008; Taradaj 2009); and insufficient information provided for at least one of the prognostic variables (e.g. data presented in categorical format which is less useful for group comparisons) (five trials) (Moffatt 1999; Meyer 2003; Moffatt 2003a; Mariani 2008; Szewczyk 2010). Scrutiny of baseline ulcer area and duration suggested imbalances which could confound the treatment effect in the 12 remaining studies (Hendricks 1985; Rubin 1990; Duby 1993; Colgan 1995; Wilkinson 1997; Danielsen 1998; Taylor 1998; DePalma 1999; Vowden 2000; O'Brien 2003; Jünger 2004b; Polignano 2004a).

\section{Effects of interventions}

\section{Review Question 1: Does the application of compression bandages or stockings aid venous ulcer healing?}

Overall, eight RCTs were identified that compared compression with no compression. These studies were grouped according to the type of comparator: primary dressing only (Eriksson 1984; Kikta 1988; Taradaj 2007); non-compressive bandages (Rubin 1990); and usual care that did not routinely include compression (Charles 1991; Taylor 1998; Morrell 1998; O'Brien 2003).

\subsection{Compression compared with primary dressing alone (three RCTs)}

Three trials compared the use of compression with primary dressings alone (Eriksson 1984; Kikta 1988; Taradaj 2007). All were small, with two classified as being at high risk of bias (Eriksson 1984; Kikta 1988), and the other at an unclear risk of bias (Taradaj 2007). Eriksson 1984 recruited 44 participants and reported mean percentage decrease in ulcer area and volume at eight weeks, which were both greater for compression. The values, as read from a figure with no available variability estimates, were: porcine skin dressing 65\% (area) and 75\% (volume); aluminium foil dressing $10 \%$ and $0 \%$; and compression $80 \%$ and $90 \%$ respectively. The estimates for the group receiving the porcine skin dressing are difficult to interpret as the randomised intervention ceased midstudy because of lack of availability of the dressing. At this point, patients in this group crossed over to the compression treatment. Six patients receiving the aluminium foil dressing discontinued treatment because of ulcer deterioration. None of the patients randomised to compression discontinued treatment.

The second study recruited 84 patients with 87 venous leg ulcers (Kikta 1988). More participants healed with Unna's boot (some compression) than hydrocolloid dressing alone though this difference was not statistically significant at six months when analysed for this review using the assumption that those lost to follow-up were unhealed: RR $1.50(95 \% \mathrm{Cl} 0.90$ to 2.50$)$, P value 0.12 (Analysis 1.1). None of the patients receiving compression discontinued treatment because of adverse events compared with 10 patients in the hydrocolloid group.

The third evaluation was a three-armed trial, but only one comparison was relevant to this review (49 participants) (Taradaj 2007). All patients were recruited post-operatively having undergone ligation and stripping of the saphenous or sagittal veins. Patients in both groups received moist normal saline dressings and pharmacotherapy in the form of a combined tablet preparation of diosmin $450 \mathrm{mg}$ and hesperidin $50 \mathrm{mg}$ ( 2 tablets daily). This preparation has been marketed as an agent to improve venous circulation (Stragen Chemical 2011; Belmont Pharmacy 2012). The group allocated compression received a two-component system comprising an elastic bandage and an outer stocking. After seven weeks of treatment, no statistically significant differences were observed between treatment groups in terms of mean percentage change in ulcer area relative to baseline: $-62.6 \%$ for the noncompression group and $-69.4 \%$ for compression, $\mathrm{P}$ value $>0.05$ (reported by trial authors). The finding was similar for mean percentage change in ulcer area per week: $-8.9 \%$ and $-9.9 \%$ respectively, $P$ value $>0.05$ (reported by trial authors). No variability estimates were provided. Withdrawals were not reported. 


\subsection{Compression compared with non-compressive bandages (one RCT)}

A multicentred RCT classified as unclear in terms of risk of bias compared Unna's boot with polyurethane foam dressing (36 participants) (Rubin 1990). All patients received elastic bandages as a retaining layer that did not provide compression. Significantly more patients completely had healed at 12 months with compression: RR 2.30 ( $95 \% \mathrm{Cl} 1.29$ to 4.10 ), P value 0.005 (Analysis 2.1). None of the patients randomised to compression withdrew from treatment, whereas nine of those allocated to the non-compressive regimen withdrew because of malodorous wound exudate. Six of these nine patients experienced an increase in ulcer size during the trial.

\subsection{Compression compared with usual care that did not routinely include compression (four RCTs)}

One UK trial compared a SSB applied by a specialist nurse with usual district nurse care (not involving compression) (Charles 1991). The other three studies compared packages of specialised leg ulcer care that included provision of the $4 \mathrm{LB}$, with usual primary care management that generally did not involve compression; two were conducted in the UK (Taylor 1998; Morrell 1998) and one in Ireland (O'Brien 2003). Two trials were classified as being at high risk of bias (Taylor 1998; Morrell 1998), whilst the other two were classified as unclear (Charles 1991; O'Brien 2003).

In the evaluation of SSB versus usual care (53 participants), more patients achieved complete healing at three months in the SSB group (71\% versus $25 \%$ ) (Charles 1991). The authors stated that this difference was statistically significant, but did not report the $P$ value (data not plotted, as raw numbers unavailable). Twenty-one per cent of the usual care group experienced an increase in ulcer area during the trial versus none in the SSB group. Three patients withdrew from each group.

Three trials compared the 4LB provided in the context of a specialist leg ulcer community service with usual management by the general practitioner (GP) and district nurse (Taylor 1998; Morrell 1998; O'Brien 2003). In one trial (36 participants), significantly more patients experienced complete healing at three months in the compression group: RR 4.0 ( $95 \% \mathrm{Cl} 1.35$ to 11.82 ), $\mathrm{P}$ value 0.01 (Analysis 3.1) (Taylor 1998). Further analyses reported in the paper suggested that healing occurred more rapidly with the 4LB. Two patients withdrew from this treatment, compared with four in the usual care group. Cost analyses based on consumables, district nurse time and mileage estimated significantly lower values for the 4 LB both per week and for the whole trial duration.

The second trial (233 participants) found no statistically significant difference in complete healing at one year (Morrell 1998): RR 1.18 (95\% $\mathrm{Cl} 0.96$ to 1.47 ), $\mathrm{P}$ value 0.12 (Analysis 3.2). However, survival analyses conducted by the trial authors suggested significantly faster time to healing for the compression group (median weeks to healing 20 versus 43, P value 0.03 , log rank test). An adjusted HR estimate was also in favour of compression: HR $1.65(95 \% \mathrm{Cl} 1.15$ to 2.35 ) ( $P$ value $<0.05$, exact value not reported). Ulcer recurrence during the one-year trial period was not significantly different between groups: RR 1.53 ( $95 \% \mathrm{Cl} 0.88$ to 2.66 ), $\mathrm{P}$ value 0.13 (Analysis 3.3) and the log rank test of difference in time to recurrence was also not significantly different between groups ( $P$ value 0.38 ). No significant differences were detected between groups either for change in health status during the trial, or for mean NHS cost per patient per year. Seventeen patients withdrew from the 4LB group and 23 from usual care.

Another RCT (200 participants) estimated shorter healing time with compression ( $P$ value $0.006, \log$ rank test and $P$ value 0.015 from adjusted Cox model) (O'Brien 2003). Costs per leg healed were significantly lower for the compression group: median (interquartile range) cost (presume price year 1999-2000) EUR 209.7 (137.5 to 269.4 ) versus EUR 234.6 (168.2 to 345.1 ), P value 0.04 . In addition, the compression group experienced statistically significant increases in some domains of health-related quality of life at six weeks relative to the usual care group, detected in both disease-specific (including global score) and generic instruments.

Findings from the three evaluations of the $4 \mathrm{LB}$ are difficult to interpret because some patients in the usual care group could have received compression, but full details (e.g. number of patients, type of compression) are not always documented. In addition, the bandage application is not the only systematic difference between the two groups; other aspects, such as the provision of specialist care to the compression groups could have influenced the outcomes.

\section{Summary of evidence for Review Question 1: Does the application of compression bandages or stockings aid venous ulcer healing?}

Overall, there is some evidence that venous ulcers heal more rapidly with compression than without. The overall risk of bias for all eight RCTs was either high or unclear. For the comparisons of compression with primary dressing alone and non-compressive bandages all the RCTs were small. The evidence for compression versus usual care (not routinely including compression) included two large trials (Morrell 1998; O'Brien 2003). Some of the observed benefits for patients receiving a specialised package of care that included application of the 4LB when compared with usual care could be explained by aspects other than compression, for example, a higher level of staff expertise resulting in better clinical management of leg ulceration overall. The evidence on relative costs of compression versus usual care was conflicting, with two trials suggesting that management with compression generated lower costs (Taylor 1998; O'Brien 2003), and one indicating no significant difference between treatment groups (Morrell 1998).

\section{Review Question 2: Which compression bandage or stocking system is the most effective?}

\subsection{Single-component compression bandage systems (seven RCTs)}

For the purposes of this review, it has been assumed that a single-component compression system consists of one type of compression bandage that may be used with, or without, a primary dressing. When used, primary dressings have been recorded, but are not considered as part of the compression system. Seven trials were identified overall. Two compared different single-component compression systems (Cordts 1992; Zuccarelli 1997), and five compared single-component systems with multicomponent compression (Eriksson 1986; Travers 1992; Colgan 1995; Kralj 1996; Nelson 2007a). 
2.1.1 Comparison between different single-component bandage systems (two RCTs)

One RCT compared a cohesive elastic bandage used in conjunction with a hydrocolloid primary dressing versus a zinc oxide and calamine paste-impregnated bandage (Unna's boot) (Cordts 1992). This trial was small (30 participants analysed) and had an overall high risk of bias. No statistically significant between-group differences were found for complete healing at 12 weeks, RR 1.17 (95\% Cl 0.54 to 2.54) (Analysis 4.1), and pain score (not plotted). Adverse events were reported in two patients receiving the elastic bandage and in three patients allocated the paste bandage, but none of these necessitated withdrawal from treatment. There were seven withdrawals in the elastic bandage group and six for the paste bandage.

In an RCT with unclear risk of bias, 48 participants were randomised to receive either an elastic bandage or a SSB for two months (Zuccarelli 1997). Bandages were removed at night in both groups and primary dressings were not described. At two months, the mean reduction in ulcer area was $3.1 \mathrm{~cm} 2$ for the elastic bandage and $1.6 \mathrm{~cm}^{2}$ for SSB (values calculated by the review authors from baseline and follow-up ulcer area data provided in the paper). The number of patients remaining in the trial at two months was not clear. No secondary outcomes were reported.

\subsubsection{Single-component bandage systems compared with multi-} component bandage systems (five RCTs)

Five trials compared a single-component compression bandage system with multi-component compression bandages. In one trial, the single-component system was an inelastic bandage (Kralj 1996), whilst all the others evaluated elastic bandages (Eriksson 1986; Travers 1992; Colgan 1995; Nelson 2007a). The multicomponent systems included two components (Eriksson 1986), three components (Travers 1992), and the 4LB (Colgan 1995; Nelson 2007a; Kralj 1996). One RCT included a third study arm where patients received four-component compression based on a pastebandage system (Colgan 1995). Three RCTs were at high risk of bias (Colgan 1995; Kralj 1996; Nelson 2007a), whilst the other two were unclear (Eriksson 1986; Travers 1992).

One trial (34 participants) compared single-component compression (elastic bandage plus hydrocolloid dressing) with two components (zinc oxide paste-impregnated stocking plus an outer elastic bandage) (Eriksson 1986). In the single-component group, the elastic bandage was removed at night and reapplied in the morning by the patient. There was no significant difference between groups at 12 weeks for complete healing: RR 1.29 (95\% $\mathrm{Cl} 0.62$ to 2.65 ) (Analysis 5.1). Two patients receiving the singlecomponent system withdrew, compared with three from the other group.

When a single-component system (elastic cohesive bandage) was compared with a three-component bandage (paste bandage, non-cohesive elastic bandage and elastic tubular overlay; 27 participants) there was no statistically significant difference detected at seven weeks for percentage change relative to baseline ulcer area (difference in means $-7.0 \%, 95 \% \mathrm{Cl}-18.38$ to 4.38 , based on values read from graph) (Analysis 5.2) (Travers 1992). There were no withdrawals.

Another trial (30 participants) evaluated three types of compression: a single-component compression system consisting of polyurethane foam primary dressing plus elastic bandage; the 4LB; and a modified Unna's boot consisting of four components (paste bandage, cotton crepe bandage, elastic adhesive bandage and class II compression sock) (Colgan 1995). In terms of complete healing at 12 weeks, no significant difference was found between groups for the comparison between single-component compression and the $4 \mathrm{LB}, \mathrm{RR} 0.33(95 \% \mathrm{Cl} 0.09$ to 1.27 ) (Analysis 5.1), or for single-component versus modified Unna's boot, RR 0.29 $(95 \% \mathrm{Cl} 0.08$ to 1.05$)$ (Analysis 6.1). There were no cases of ulcer recurrence during a six month follow-up period. Three participants withdrew from the single-component group, one from the Unna's boot group and none from the 4LB group. The average cost of the bandages per participant over 12 weeks in IEP (Irish pounds) (price year not stated) was: single-component IEP 58.33, Unna's boot IEP 66.24, and 4LB IEP 82.54

A large trial (245 participants) with $2 \times 2 \times 2$ factorial design evaluated pentoxifylline versus placebo, knitted viscose versus hydrocolloid dressings as well as single-component compression (hydrocolloid-lined elastic adhesive bandage) versus the 4LB (Nelson 2007a). Initially analyses were conducted on all participants, i.e. those with both simple and non-simple venous ulceration (non-simple defined as serologically confirmed rheumatoid arthritis or venous pathology not confirmed with handheld Doppler). The estimate for complete healing at 24 weeks suggested a statistically significant difference in favour of the 4LB over the single-component bandage: RR $0.74(95 \% \mathrm{Cl} 0.59$ to 0.92) (Analysis 5.1). A Kaplan-Meier estimate of median days to healing showed faster wound closure for the 4LB group (78 versus 168 days, log rank test not reported) and a HR estimate from an adjusted Cox proportional hazards model was 2.0 (95\% $\mathrm{Cl} 1.4$ to 2.9 ), $\mathrm{P}$ value $<0.0005$, indicating a greater probability of healing with the $4 \mathrm{LB}$. The proportion of participants who changed bandage during the trial because of an adverse event was $28 \%$ for the single-component compression and $15 \%$ for the 4 LB. Further analyses (complete healing, withdrawal rate and quality of life) were conducted on a subset of participants with simple venous ulceration (200 participants). The estimate for complete healing at 24 weeks was similar to that for the total study population: RR $0.70(95 \% \mathrm{Cl} 0.55$ to 0.89$)$ (Analysis 5.3). The proportion of participants who withdrew from the bandage system with or without simultaneous withdrawal from the randomised drug and dressing treatment was $20 \%$ for the single-component group and $5 \%$ for the 4LB. Health-related quality of life was assessed using the Nottingham Health Profile and showed significantly greater improvements in some domains for the 4LB group at 24 weeks.

A small trial (40 participants) found similar rates of complete healing at six months with an inelastic bandage (used with a hydrocolloid primary dressing) and the 4LB (Kralj 1996): RR 1.14 (95\% Cl 0.51 to 2.55 ), $\mathrm{P}$ value 0.74 (Analysis 5.1). Mean days to healing were 57.6 for the $4 \mathrm{LB}$ and 84.9 for the inelastic bandage (statistical methods of deriving these values not stated). Four participants withdrew from the 4LB group and two from the singlecomponent group.

Analysis 5.1 includes outcomes for four trials reporting complete healing. Estimates have not been pooled because of differences in interventions and length of follow-up. 


\section{Summary of evidence for Section 2.1: Single-component compression systems}

No differences were found between different types of singlecomponent compression evaluated in two small RCTs. Findings from the largest trial suggested better healing outcomes for the 4LB compared with single-component compression in terms of frequency of complete healing and time to healing. In addition, adverse event rates were lower and quality of life scores higher for the 4LB. The other four RCTs (all small) involving a comparison with multi-component compression did not detect significant differences between groups for healing outcomes. All RCTs had high or unclear risk of bias.

\subsection{Two-component compression bandage systems (six RCTs)}

Of six trials identified, two compared alternative two-component systems (Danielsen 1998; Moody 1999), and four compared twocomponent systems with the 4LB (Moffatt 2003a; Harley 2004; Moffatt 2008; Szewczyk 2010).

\subsubsection{Comparison between different two-component bandage systems (2 RCTs)}

Two trials compared elastic and inelastic (SSB) outer bandages placed over padding of the lower limb (Danielsen 1998; Moody 1999). Both had unclear risk of bias. When data were pooled for complete healing at three to six months (95 participants), there was no statistically significant difference between groups: RR 1.23 (95\% $\mathrm{Cl} 0.67$ to 2.25 ), $\mathrm{P}$ value 0.51 (test for heterogeneity $\mathrm{P}$ value $0.47, \mathrm{I}$ $=0 \%$ ) (Analysis 7.1).

In the first trial (43 participants), complete healing was also reported at one month and one year (Danielsen 1998). At one month, there was no statistically significant difference between the alternative two component systems, RR 3.48 ( $95 \% \mathrm{Cl} 0.42$ to 28.63 , Analysis 7.2), however, at one year more people had healed in the system with the elastic outer bandage: RR $3.48(95 \% \mathrm{Cl} 1.14$ to 10.60), $P$ value 0.03 (Analysis 7.3). Also, a Kaplan-Meier estimate of proportions healed at one year were $81 \%$ for the elastic bandage and $31 \%$ for SSB (P value 0.03).

The second trial (52 participants) reported mean times to healing of 9.3 weeks for the group receiving an elastic bandage and 9.9 weeks for the SSB (Moody 1999). The percentages of participants with increased ulcer size and clinical infection during the study period were $23 \%$ and $15 \%$ respectively for those receiving an elastic bandage and $15 \%$ and $12 \%$ for SSB. One participant receiving SSB withdrew, but there were no withdrawals reported for those receiving the elastic bandage.

\subsubsection{Two-component bandage systems compared with the 4LB (four RCTs)}

Four RCTs compared two-component compression (consisting of a padding or cushioning layer followed by an elastic bandage) with the 4LB (Moffatt 2003a; Harley 2004; Moffatt 2008; Szewczyk 2010). One RCT was at high risk of bias (Moffatt 2003a), and the other three were unclear (Harley 2004; Moffatt 2008; Szewczyk 2010).

One cross-over RCT (81 participants) of eight weeks' duration stipulated the primary outcome for the trial as degree of bandage slippage (Moffatt 2008). Complete healing was assessed at four weeks, just prior to the cross-over, and there were no statistically significant difference between groups: RR $2.15(95 \% \mathrm{Cl} 0.58$ to
8.03) (Analysis 8.1). In terms of adverse events assessed over the whole 8-week trial period, 68 occurred whilst using the twocomponent system and 67 with the 4LB. Prior to cross-over, two participants withdrew from the group receiving two-component compression (both due to adverse events) and one withdrew from the group allocated the 4LB (participant request). Healthrelated quality of life was assessed using the Cardiff Wound Impact Schedule. During the first four weeks, no significant betweengroups differences were detected for overall health-related quality of life. However, significant differences were observed in favour of the two-component system for one domain, i.e. physical symptoms and daily living, ( $P$ value $<0.05$, based on per protocol analysis by trial authors).

The second and largest of these trials (112 participants) reported a statistically significant difference in favour of the 4 LB for complete healing at six months when participants were analysed up to end of the randomised treatment: RR $0.56(95 \% \mathrm{Cl} 0.41$ to 0.77 ), P value 0.0003 (Analysis 8.3) (Moffatt 2003a). This analysis was repeated, this time including participants who had healed following withdrawal from the randomised treatment, some of whom switched bandage systems; the between group difference was not statistically significant: RR $0.88(95 \% \mathrm{Cl} 0.73$ to 1.05$)$ (Analysis 8.4). The adjusted HR was 1.18 (95\% Cl 0.69 to 2.02), $\mathrm{P}$ value 0.55 . In the group receiving the two-component system, 19 participants reported 21 bandage-related adverse events compared with seven participants with eight events in the 4LB group. Withdrawal rates were $54 \%$ for the two-component system and $12 \%$ for $4 \mathrm{LB}$. The mean weekly cost of treatment per participant (clinic costs and home care costs) was lower in the 4LB group (GBP 79.91 versus GBP 83.56) and the same trend was observed for mean cost per participant over the six-month trial (GBP 876 versus GBP 916) (price year 2000). Assessment of health-related quality of life using SF-36 data at 24 weeks and at healing/withdrawal adjusted for baseline score suggested no significant differences between groups.

The third trial recruited 30 participants and reported a shorter average time on treatment for the group receiving the twocomponent compression (63 versus 87 days, difference described as not statistically significant but no $P$ value or confidence interval presented) (Harley 2004). The methods used for estimating the time on treatment were not explained, and it was not clear whether mean or median values were reported. A chi-squared analysis of quintiles of healing times suggested no statistically significant association between treatment group and healing duration ( $P$ value 0.7$)$, but this is not surprising given the very small numbers of participants in each time interval category per treatment group (maximum of five participants). In relation to adverse events, outcomes were better for participants treated with the 4LB for: the number of participants reporting at least one adverse event (63\% versus $100 \%)$; average number of adverse events per participant (1.64 versus 0.75 ); and average number of incidents of inappropriate pressure (2.31 versus 5.43) (all differences were described as significant but $P$ values and confidence intervals were not provided). Withdrawal rates favoured the 4 LB ( $6 \%$ versus $43 \%$, $P$ value 0.018$)$; reasons for withdrawal were not provided. The estimated costs of six week's treatment excluding dressings was AUD 35.00 for the two-component system and AUD 114.00 for the 4LB (price year appeared to be 1999 to 2000). The trial follow-up period was unclear (possibly three months). 
The fourth trial (31 participants) compared a compression stocking with a two-component bandage system and the 4LB (Szewczyk 2010); comparisons between the two types of bandage and the stocking are considered later (Section 2.6.3). No statistically significant differences were detected between groups at three months for mean percentage reduction in ulcer area $(98 \%$ for the group receiving the two-component bandage system versus $94 \%$ for the 4LB) or mean healing rate $\left(0.55\right.$ and $0.63 \mathrm{~cm}^{2}$ per week respectively). No variance data were provided for either outcome. No secondary outcomes were reported.
Data from three of the four trials were pooled for the outcome of complete healing at three months (170 participants) (Moffatt 2003a; Harley 2004; Szewczyk 2010), the estimate suggested no statistically significant difference in healing between twocomponent systems and the 4LB: RR 0.83 (95\% $\mathrm{Cl} 0.66$ to 1.05), $\mathrm{P}$ value 0.12 (test for heterogeneity $P$ value $0.59,12=0 \%$ ) (Analysis 8.2 , Figure 5). Data from Moffatt 2008 were not included in the pooled analysis because of the shorter follow-up period (4 weeks).

Figure 5. Forest plot of comparison: 8 Two-component system vs four-layer bandage (4LB), outcome: 8.2 Patients with complete healing at 3 months.

\begin{tabular}{|c|c|c|c|c|c|c|c|c|c|}
\hline \multirow[b]{2}{*}{ Study or Subgroup } & \multicolumn{2}{|c|}{ Two-component system } & \multicolumn{2}{|c|}{ Four-layer bandage (4LB) } & \multirow[b]{2}{*}{ Weight } & \multirow{2}{*}{$\begin{array}{c}\text { Risk Ratio } \\
\text { M-H, Fixed, 95\% Cl }\end{array}$} & \multirow{2}{*}{\multicolumn{2}{|c|}{$\begin{array}{c}\text { Risk Ratio } \\
\text { M-H, Fixed, } 95 \% \mathrm{Cl}\end{array}$}} & \\
\hline & Events & Total & Events & Total & & & & & \\
\hline Harley 2004 & 8 & 14 & 13 & 16 & $20.4 \%$ & $0.70[0.42,1.17]$ & & & \\
\hline Moffatt 2003a & 30 & 52 & 40 & 57 & $64.0 \%$ & $0.82[0.62,1.10]$ & & & \\
\hline Szewczyk 2010 & 10 & 16 & 9 & 15 & $15.6 \%$ & $1.04[0.59,1.83]$ & & & \\
\hline Total $(95 \% \mathrm{Cl})$ & & 82 & & 88 & $100.0 \%$ & $0.83[0.66,1.05]$ & & & \\
\hline Total events & 48 & & 62 & & & & & & \\
\hline $\begin{array}{l}\text { Heterogeneity: } \mathrm{Chi}^{2}= \\
\text { Test for overall effect }\end{array}$ & $\begin{array}{l}1.04, d f=2(P= \\
Z=1.57(P=0\end{array}$ & $\left.\right|^{2}=0 \%$ & & & & & $\begin{array}{rr}0.1 & 0.2 \\
& F\end{array}$ & $\begin{array}{ccc}0.5 & 1 & 2 \\
\text { vours } 4 \text { LB } & \text { Favours }\end{array}$ & $\begin{array}{c}5 \\
50 \text {-compon }\end{array}$ \\
\hline
\end{tabular}

\section{Summary of evidence for Section 2.2: Compression systems comprising two components}

When alternative two-component compression systems were compared (the difference being elastic and inelastic outer bandages), there was no evidence of a between-group difference for complete healing at three to six months (Danielsen 1998; Moody 1999). Further findings from one RCT suggested a better performance for the system including an elastic bandage in terms of complete healing at one year (Danielsen 1998). Both trials were small with unclear risk of bias.

Pooled data from three trials for the outcome of complete healing at three months suggested no significant difference between twocomponent compression and the 4LB (Moffatt 2003a; Harley 2004; Szewczyk 2010). There was some evidence to suggest fewer adverse events and fewer withdrawals for the 4LB (Moffatt 2003a; Harley 2004) although groups were similar with respect to both these outcomes in another RCT (Moffatt 2008). Moffatt 2008 reported findings in favour of the two-component compression for quality of life changes i.e. physical symptoms and daily living. Harley 2004 reported that costs were greater for the $4 \mathrm{LB}$, however, a detailed economic evaluation was not provided. Risk of bias was high for one RCT (Moffatt 2003a), and unclear for the other three (Harley 2004; Moffatt 2008; Szewczyk 2010).

\subsection{Three-component compression bandage systems (four RCTs)}

\subsubsection{Comparison between different three-component bandage} systems

Four trials compared alternative three-component compression systems (Duby 1993; Callam 1992b; Gould 1998; Meyer 2002). One
RCT was at high risk of bias (Gould 1998), and the other three were unclear. In three RCTs, treatment groups were mainly distinguished by the middle component being an elastic or inelastic bandage (Callam 1992b; Gould 1998; Meyer 2002). The fourth trial compared a SSB system with a paste-bandage system (Duby 1993).

Pooling of two RCTs suggested significantly more participants (Callam 1992b), and ulcers (Gould 1998), were completely healed by three to four months when the compression system incorporated an elastic - rather than inelastic - bandage (171 participants/ulcers): RR 1.83 ( $95 \% \mathrm{Cl} 1.26$ to 2.67), P value 0.002 (test for heterogeneity $P$ value $0.75,12=0 \%$ ) (Analysis 9.1; Figure 6 ). The possibility of highly correlated healing data influencing the estimate of effect should be noted in the trial that used ulcers as the unit of randomisation/ analysis (Gould 1998). The third RCT (112 participants) did not detect a statistically significant difference in healing at six months (Meyer 2002): RR 0.94 (95\% Cl 0.69 to 1.27), P value 0.67 (Analysis 9.1; Figure 6), and reported similar median time to healing for both groups: 9.0 versus 9.5 weeks for groups receiving elastic and inelastic middle components, respectively. One RCT reported that two participants in each group had a minor degree of damage related to the bandage (Callam 1992b), and also reported that a greater proportion of those receiving the inelastic component complained of ulcer pain at all clinic visits ( $48 \%$ versus $29 \%$, P value 0.03). Callam 1992b reported that more participants discontinued treatment in the group receiving the inelastic middle component (30\% versus $12 \%$ ), whilst Meyer 2002 presented similar withdrawal rates for both groups (around 14\%), and Gould 1998 did not provide data per group. 
Figure 6. Forest plot of comparison: 93 components including elastic bandage vs 3 components including inelastic bandage, outcome: 9.1 Patients/limbs with complete healing during trial.

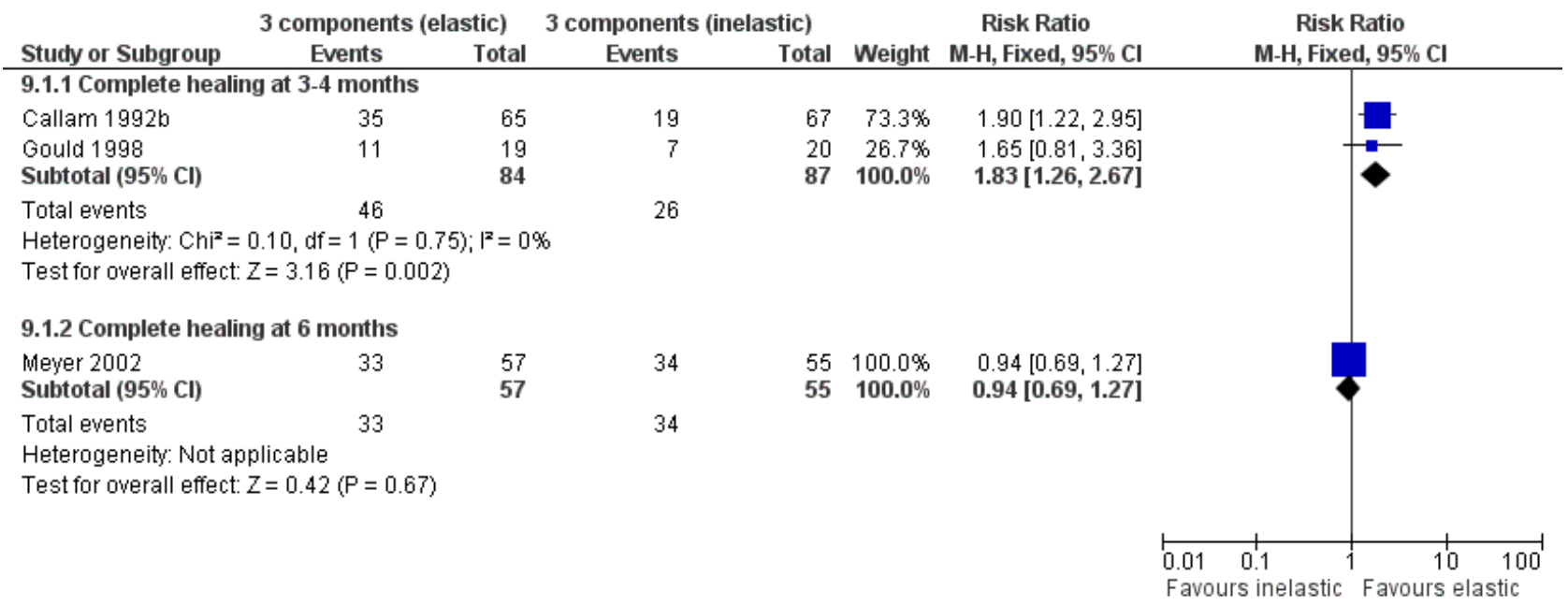

The fourth RCT (51 limbs) suggested no statistically significant difference between the groups in complete healing at three months: RR 1.73 (95\% Cl 0.74 to 4.06), P value 0.20 (Analysis 10.1 ) (Duby 1993). Since limbs rather than participants were allocated, and this was not adjusted for in any analyses, the possibility of biased estimates of treatment effect should be considered.

\section{Summary of evidence for Section 2.3: Compression systems comprising three components}

Four RCTs compared alternative three-component compression bandage systems (Duby 1993; Callam 1992b; Gould 1998; Meyer 2002). In three, the main difference between study arms was whether the middle component was elastic or inelastic (Callam 1992b; Gould 1998; Meyer 2002). A pooled estimate from two trials for complete healing at three to four months suggested a better outcome for the system including an elastic bandage (Callam 1992b; Gould 1998). The third trial found no difference between groups for complete healing at six months and median times to healing (Meyer 2002). A fourth trial that compared short-stretch and paste-bandage systems did not report any significant differences between groups (Duby 1993). One trial was considered to be at high risk of bias (Gould 1998), while risk of bias was unclear for the other three (Duby 1993; Callam 1992b; Meyer 2002).

\subsection{Compression systems comprising four components including an elastic component (the ' $4 L B$ ')}

\subsubsection{Comparison between different versions of the 4LB (three RCTs)}

Three trials compared variants of the 4LB (Wilkinson 1997; Moffatt 1999; Vowden 2000). Two were at high risk of bias (Wilkinson 1997; Vowden 2000), and the other was at unclear risk of bias (Moffatt 1999). Data from two trials were plotted for the comparison of the original Charing Cross 4LB with an alternative system, but were not pooled as the comparators differed. Individual trial estimates showed no statistically significant differences at three months for complete healing (participants or limbs) (Wilkinson 1997; Moffatt 1999), or at six months in one evaluation (232 participants): RR 0.96 (95\% Cl 0.83 to 1.12), P value 0.6 (Moffatt 1999) (Analysis 11.1). Moffatt 1999 also reported an adjusted HR estimate of $1.18(95 \% \mathrm{Cl}$ 0.87 to 1.59$)$, $P$ value 0.28
The third RCT (149 participants) compared three different fourcomponent systems: the original Charing Cross 4LB; a modified 4LB (alternative devices were substituted for the two middle components, but appeared to have similar characteristics to the originals); and a proprietary kit (Robinson Ultra-Four) (Vowden 2000). No statistically significant differences between groups were reported for complete healing, respective rates being $60 \%, 76 \%$ and $60 \%$ at three months, and $87 \%, 84 \%$ and $83 \%$ at five months (data not plotted as raw numbers unclear).

Moffatt 1999 reported similar health-related quality of life scores (Nottingham Health Profile) between groups for all domains at six months. A small number of withdrawals because of bandage discomfort were noted for all three trials and there were no apparent differences between treatment groups. Two trials reported pressure damage arising from the bandage: this affected one participant in each of the two arms receiving alternatives to the original Charing Cross system (Vowden 2000), and one participant receiving the Charing Cross system (Wilkinson 1997).

\section{Summary of evidence from Section 2.4.1: Comparison between different versions of the $4 L B$}

Overall, there is no evidence of a difference in outcomes between different versions of the 4LB system from three RCTs with high or unclear risk of bias.

\subsubsection{LB compared with multi-component systems including an inelastic bandage (the SSB) (six RCTs)}

Individual patient data meta-analysi

Six RCTs were identified from database searches that compared the 4LB with a multi-component system that included a SSB (Duby 1993; Scriven 1998; Partsch 2001; Ukat 2003; Franks 2004; Iglesias 2004). In these RCTs, the 4LB systems all comprised orthopaedic wool, crepe bandage, an elastic bandage and an elastic cohesive bandage as the outer layer. The comparator systems usually consisted of orthopaedic wool, one or two SSBs and sometimes a retaining layer (e.g. a cohesive bandage or tubular device). An additional eligible unpublished trial was identified at a wound management conference. These seven trials recruited 887 participants in total. Patient-level data were unavailable for Duby 
1993 and the unpublished trial; these two RCTs recruited 83/887 $(9.4 \%)$ of the eligible participants. Table 1 shows details of all seven RCTs.

In terms of the five RCTs with available IPD, ten participants were excluded from the original investigators' own analyses $(1.1 \%$ of known randomised participants), of whom three were reinstated by the review authors for the meta-analysis. Data for the seven other participants were not available (Table 1). In total, data from 797 participants were included in the meta-analysis. Table 2 shows participants' characteristics for the five trials with available IPD (89.8\% of known randomised participants); most participants were ambulant. The reported follow-up periods for RCTs ranged from three to 12 months. The overall median follow-up of participants who did not heal during the trial period was around 13 weeks (estimate derived from IPD, Table 1).

Four of the five RCTs with available IPD were classified as being at overall high risk of bias because of non-blinded outcome assessment (Scriven 1998; Partsch 2001; Ukat 2003; Franks 2004). The remaining RCT was deemed to be at low risk of bias, confirming healing from photographs at the trial office by an investigator blinded to treatment allocation (Iglesias 2004). Checks on the IPD for each RCT suggested that baseline comparability was generally satisfactory and that data were mostly complete, free of duplication, consistent and feasible. The small number of queries raised were resolved through discussion with the relevant trialist.
The results of model-checking procedures indicated that the proportional hazards assumption was upheld for all potentially predictive covariates. The only continuous covariate included in the final models (baseline ulcer surface area) was entered as a natural log transformation. Outcomes were similar for early and late accruals when we took into account differences between study centres in four trials assessed (Iglesias 2004; Partsch 2001; Ukat 2003; Franks 2004). To assess the adequacy of model fit, we assessed the number of events (an event being a healed wound) against the number of covariates entered at the start of each model. At least 10 events per variable are required in logistic regression to reduce bias in regression coefficients (Peduzzi 1996). All models generated from the meta-analysis data set met this recommendation.

\section{Primary outcomes: findings from preliminary analyses of IPD}

When data were pooled for the outcome of complete healing (unadjusted) during the trial period (797 participants from five RCTs), no significant difference between treatment groups was observed: fixed-effect RR $0.96(95 \% \mathrm{Cl} 0.88$ to 1.05$)$, P value 0.34 (chi-squared test for heterogeneity $\mathrm{P}$ value $0.54, \mathrm{I} 2=0 \%$ ) (Analysis 12.1, Figure 7). For the trial without available IPD, complete healing at three months was similar for the 4LB and the SSB (NB unit of analysis was limbs, not participants): RR 1.10 (95\% Cl 0.57 to 2.11), P value 0.77 (Analysis 12.2; Duby 1993).

Figure 7. Forest plot of comparison: 12 4LB vs multi-layer short-stretch bandage (SSB), outcome: 12.1 Patients with complete healing during trial period based on IPD.

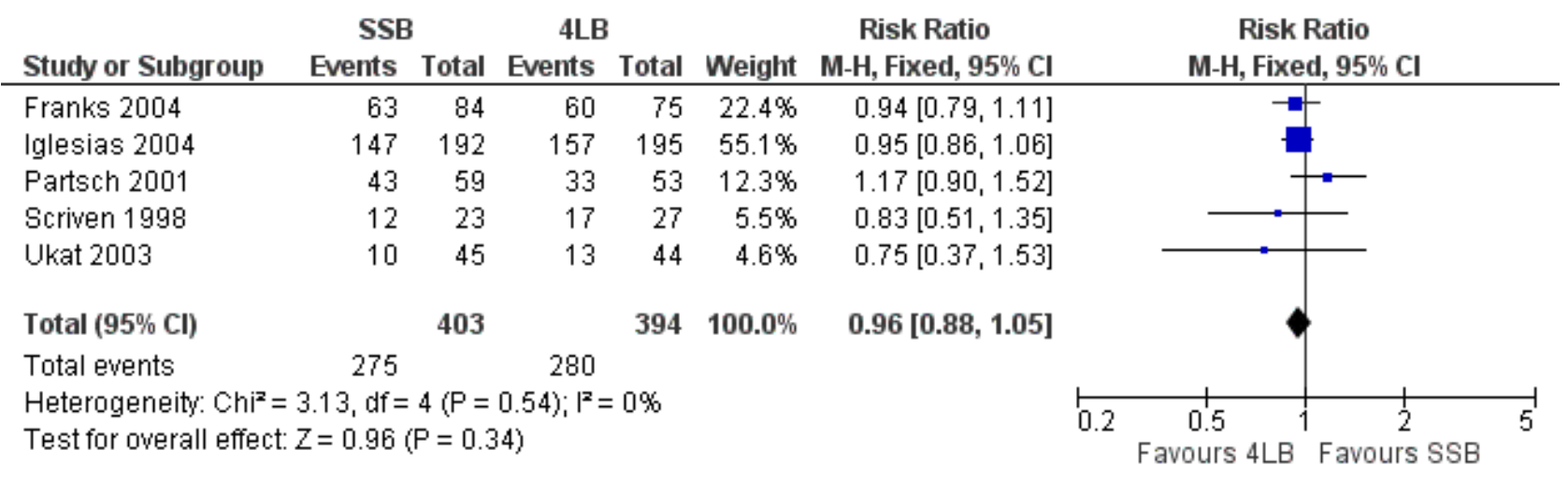

The median time to healing (based on IPD) estimated from unstratified Kaplan-Meier survival analysis (797 participants) was 90 days $(95 \% \mathrm{Cl} 79$ to 101$)$ for the $4 \mathrm{LB}$ and 99 days ( $95 \% \mathrm{Cl} 82$ to 116$)$ for the SSB, $\mathrm{P}$ value 0.133 for logrank test for difference between survival curves (Figure 8). 
Figure 8. 4LB versus SSB: Kaplan-Meier survival curves (unstratified analysis) based on IPD

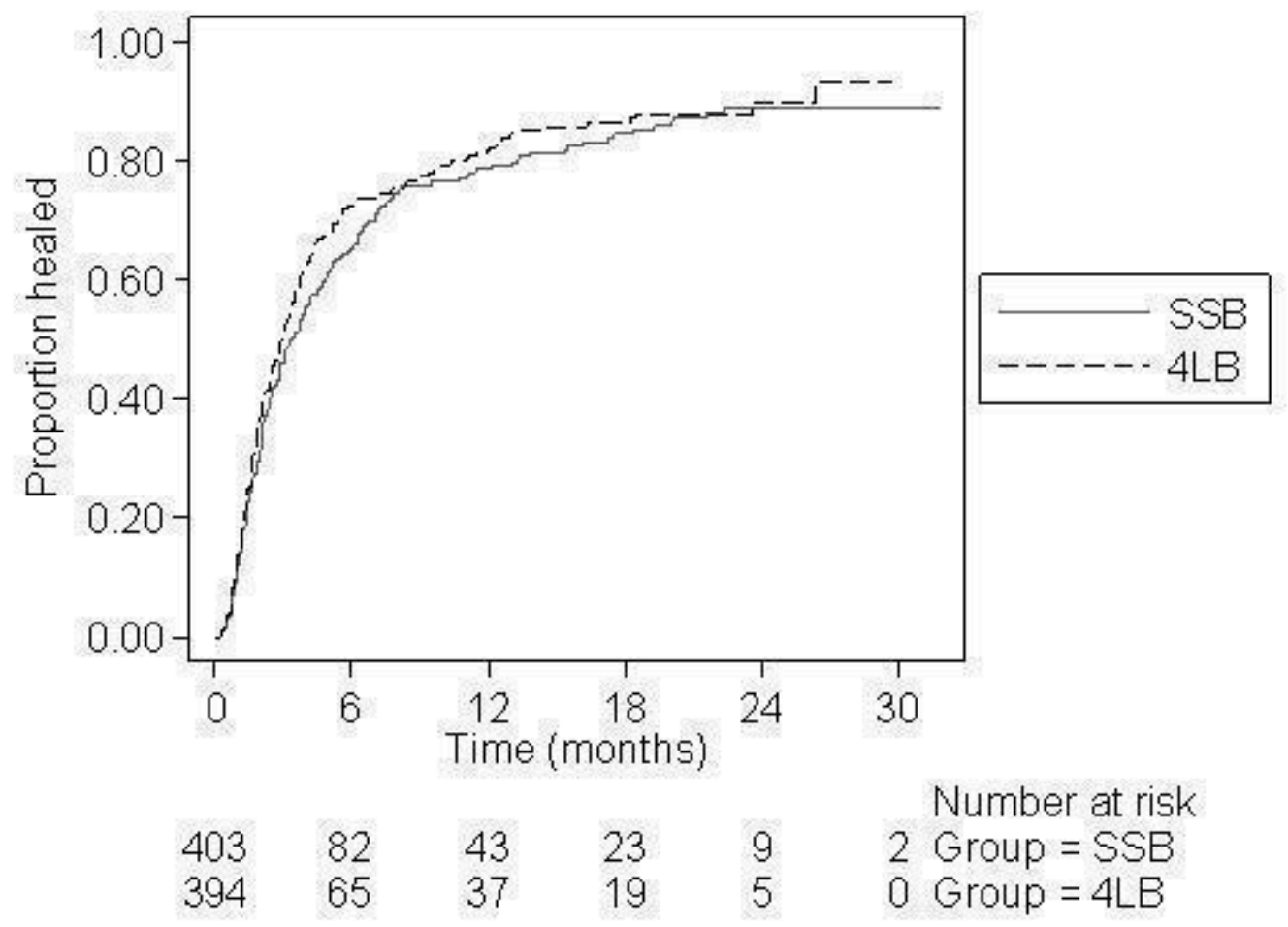

An initial Cox proportional hazards model was generated from the IPD with time to healing (days) as the dependent variable, healing as the event, study centres as strata, and bandage type as the only covariate. The result of this unadjusted analysis indicated no significant difference between bandage types: HR 1.15 (95\% Cl 0.97 to 1.37$)$, $P$ value 0.11 .

\section{Primary outcomes: findings from main analyses of IPD}

The final Cox model contained type of bandage, ulcer duration, and ulcer area (Table 3). The HR for bandage type was 1.31 (95\% Cl 1.09 to 1.58 ), $\mathrm{P}$ value 0.005 , indicating that the estimated probability of healing with the 4LB was around 1.3 times that of healing with the SSB, assuming similar values for other covariates. There was significant evidence that larger ulcers $(P$ value $<0.001)$ and ulcers of longer duration ( $P$ value $<0.001)$ predicted longer time to healing independently of one another and of treatment. The chance of healing was reduced by a factor of 0.44 for each 10 -fold increase in area. Baseline ulcer duration was divided into the following four categories: a month or less; more than one month up to and including six months; more than six months up to and including
12 months; and over 12 months. The data suggested that the hazard of healing was reduced for each step up to a longer duration interval. We found no significant interactions between bandage and baseline ulcer area, and bandage and baseline ulcer duration.

Analysis 12.3 and Figure 9 illustrate the relative contribution of each RCT to the meta-analysis, showing HR estimates for each trial individually and for all trials when pooled. The estimates were derived from the IPD and were adjusted for baseline ulcer area and baseline ulcer duration. The pooled HR estimate was very close to that derived from the Cox regression: fixed-effect HR 1.32 (95\% $\mathrm{Cl} 1.09$ to 1.60$) \mathrm{P}$ value 0.004 . Some heterogeneity between trials was detected: chi-squared $P$ value $0.11,12=48 \%$. When the analysis was repeated using a random-effects model no statistically significant difference was detected between bandages: HR 1.30 ( $95 \% \mathrm{Cl} 0.94$ to 1.80 ) P value 0.11 (Analysis 12.4). Visual inspection of the forest plot suggested that one trial was likely to be the source of heterogeneity (Partsch 2001). When this trial was removed, a sensitivity analysis showed the heterogeneity was eliminated and the observed treated effect was in favour of the 4LB in both fixedeffect (Analysis 12.5) and random-effects models (Analysis 12.6). 
Figure 9. Forest plot of comparison: 12 4LB vs multi-layer short-stretch bandage (SSB), outcome: 12.3 Hazard ratio estimates for time to healing based on IPD (fixed-effect).

\begin{tabular}{|c|c|c|c|c|c|c|c|c|c|c|}
\hline Study or Subgroup & log[Hazard Ratio] & SE & $\begin{array}{l}\text { 4LB } \\
\text { Total }\end{array}$ & $\begin{array}{l}\text { SSB } \\
\text { Total }\end{array}$ & Weight & $\begin{array}{l}\text { Hazard Ratio } \\
\text { IN, Fixed, } 95 \% \mathrm{CI}\end{array}$ & & $\begin{array}{r}\text { Hazard } \\
\text { IV, Fixed }\end{array}$ & $\begin{array}{l}\text { d Ratio } \\
\text { d, } 95 \% \mathrm{Cl}\end{array}$ & \\
\hline Franks 2004 & 0.439 & 0.272 & 75 & 84 & $12.8 \%$ & $1.55[0.91,2.64]$ & & & & \\
\hline Iglesias 2004 & 0.349 & 0.123 & 195 & 192 & $62.7 \%$ & $1.42[1.11,1.80]$ & & & & \\
\hline Partsch 2001 & -0.347 & 0.256 & 53 & 59 & $14.5 \%$ & $0.71[0.43,1.17]$ & & & & \\
\hline Scriven 1998 & 0.438 & 0.417 & 27 & 23 & $5.5 \%$ & $1.55[0.68,3.51]$ & & & & \\
\hline Ukat 2003 & 0.7 & 0.454 & 44 & 45 & $4.6 \%$ & $2.01[0.83,4.90]$ & & & & \\
\hline Total $(95 \% \mathrm{Cl})$ & & & 394 & 403 & $100.0 \%$ & $1.32[1.09,1.60]$ & & & & \\
\hline \multicolumn{7}{|c|}{$\begin{array}{l}\text { Heterogeneity: } \mathrm{Chi}^{2}=7.65, \mathrm{df}=4(\mathrm{P}=0.11) ;\left.\right|^{2}=48 \% \\
\text { Test for overall effect: } Z=2.88(P=0.004)\end{array}$} & \begin{tabular}{|l|l|}
0.10 .2 \\
$\quad$ Favo
\end{tabular} & $\begin{array}{c}0.5 \\
\text { burs SSB }\end{array}$ & $\begin{array}{l}1 \\
\text { Favours } 4 \mathrm{~L}\end{array}$ & 10 \\
\hline
\end{tabular}

The Cox proportional hazards model was re-run on a subset of four trials (747 participants) for which additional covariates were available: primary or recurrent ulceration and participants' mobility (Iglesias 2004; Partsch 2001; Ukat 2003; Franks 2004). The final model contained bandage type, ulcer area, ulcer duration and primary or recurrent ulceration. The estimated HR for type of bandage was similar to the model based on five trials: $1.29(95 \% \mathrm{Cl}$ 1.06 to 1.57 ), $P$ value 0.011 . The model suggested that larger ulcers ( $P$ value $<0.001)$, ulcers of longer duration $(P$ value $<0.001)$ and previous ulceration ( $P$ value $<0.005$ ) were independent predictors of longer time to healing (Table 4). No significant interaction was found between: bandage and baseline ulcer area; bandage and baseline ulcer duration; and bandage and primary or recurrent ulceration.

Secondary outcomes: findings from IPD - adverse events and recurrence

IPD for adverse events were retrieved for two trials (Iglesias 2004; Franks 2004). For incidence of any type of adverse event, the pooled OR (fixed-effect) was 1.15 ( $95 \% \mathrm{Cl}: 0.81$ to 1.62$), \mathrm{P}$ value 0.43 , providing no evidence of a difference between bandage types (Analysis 12.7). Some heterogeneity was detected (chi-squared test $P$ value $0.24,1^{2}=28 \%$ ) and so the analysis was repeated using a random-effects model which showed a similar estimate: OR 1.11 (95\% $\mathrm{Cl} 0.72$ to 1.72$)$, P value 0.64 (Analysis 12.8).

The two trials differed in their definitions of bandage-related adverse events. In one, maceration, allergic reaction, eczema of peri-ulcer skin and infection were coded as bandage-related (Iglesias 2004). The second trial, comparing primary dressings as well as bandages, attributed these events to dressings (Franks 2004). In view of this difference, data were not pooled. ORs were estimated for each trial individually and neither demonstrated a statistically significant between-group difference: 1.41 (95\% Cl: 0.94 to 2.11) (Iglesias 2004), and 0.78 (95\% Cl: 0.30 to 2.04) (Franks 2004) (Analysis 12.9).

Analysis of the number of all types of adverse events per participant did not show a difference between the two bandage systems: pooled difference in means (fixed-effect) $-0.10(95 \% \mathrm{Cl}-0.32$ to 0.12 ), $P$ value 0.38 (Analysis 12.10). Since some heterogeneity was detected (chi-squared test $P$ value $0.12,12=58 \%$ ) the meta-analysis was repeated using a random-effects model which generated a similar estimate: difference in means $-0.21(95 \% \mathrm{Cl}-0.68$ to 0.27$), \mathrm{P}$ value 0.39 (Analysis 12.11).
Data were not pooled for the number of adverse events related to bandaging for the reasons mentioned above. No significant differences were detected between groups for the trials individually: difference in means $-0.45(95 \% \mathrm{Cl}-1.01$ to 0.11$)$ (Iglesias 2004), and 0.04 (-0.09 to 0.17) (Franks 2004) (Analysis 12.12).

Data on time to recurrence were provided for one RCT (Iglesias 2004); the trial authors had previously published a KaplanMeier survival analysis for recurrence. No statistically significant difference was detected between the survival curves for time to recurrence for 4 LB and SSB ( 27 versus 29 recurrences respectively, log rank test $P$ value 0.22 ). No further analyses were performed by the original investigators due to the low event rate precluding exploration of the impact of covariates. Since the meta-analysis data set did not contain any additional information on time to recurrence relative to this trial's findings, no further analyses were undertaken.

Secondary outcomes: findings from aggregate data - health-related quality of life, costs and withdrawals

Two trials included an assessment of quality of life (Franks 2004; Iglesias 2004). One used the Nottingham Health Profile and observed no statistically significant differences between treatment groups for scores for any domain at six months (Franks 2004). The other trial had a large amount of missing data for this outcome and so reported a descriptive analysis of findings obtained using the SF-12 and the Hyland Leg and Foot Ulcer Questionnaire (Iglesias 2004). Overall, there did not appear to be marked differences between treatment groups.

Three trials included an analysis of costs (Scriven 1998; Ukat 2003; Iglesias 2004), but only one reported a rigorously conducted costeffectiveness analysis (Iglesias 2004). Cost estimates were based on NHS and Personal Social Services costs and health benefits were measured as differences in ulcer-free days and quality-adjusted life years (QALYs). The following estimates were reported, all in favour of the 4LB: mean between-group difference in healing time 10.9 days $(95 \% \mathrm{Cl}-6.8$ to 29.1$)$; difference in mean in QALYs -0.02 (95\% Cl -0.08 to 0.04 ); and difference in mean in total cost (price year 2001): GBP 227.32 ( $95 \% \mathrm{Cl} 16.53$ to 448.30 ) per patient per year. Sensitivity analyses showed the cost-effectiveness estimate to be robust to variation in the number of bandages used and unit costs of compression systems. The 4LB emerged as the dominant treatment strategy. The second trial calculated the cost per patient and cost per ulcer healed, based on costs of bandages and other 
disposables (e.g. primary dressings, wadding) and 30 minutes of nursing time per bandage change (Ukat 2003). Costs per patient were EUR 587 for the 4LB and EUR 1345 for the SSB; and per ulcer healed EUR 1845 and EUR 5502 respectively. Statistical tests for between group differences and price year were not reported. The third trial estimated the cost of treatment over six months as GBP 392.60 for the 4LB and GBP 184.56 for the SSB (estimates based on cost of bandage systems only, price year not stated) (Scriven 1998).

Five trials reported on withdrawals (Scriven 1998; Partsch 2001; Ukat 2003; Franks 2004; Iglesias 2004). Two found similar withdrawal rates for both treatment groups: $18 \%$ for Ukat 2003, and around 22\% for Franks 2004. Two others found higher withdrawal rates in participants allocated to the SSB: $3 \%$ versus $6 \%$ (represents proportion of limbs withdrawn) (Scriven 1998); and 24\% versus $34 \%$ (Iglesias 2004). The fifth trial reported more withdrawals in the group receiving the 4 LB, $12 \%$ versus $23 \%$ (Partsch 2001).

\section{Summary of evidence from Section 2.4.2: Comparison between the 4 LB and multi-component systems that include an inelastic bandage (the SSB)}

Analysis of IPD from five trials indicated that the estimated probability of healing with the 4LB was around 1.3 times that of healing with the SSB (statistically significant difference). One trial included a rigorous cost-effectiveness analysis that indicated that the 4LB was the dominant (more cost-effective) treatment strategy (Iglesias 2004). The adverse event profile, recurrence rates and changes in health-related quality of life scores were similar for the two bandage types. Withdrawal rates were approximately similar between groups. Risk of bias was high in four RCTs because of nonblinded outcome assessment (Scriven 1998; Partsch 2001; Ukat 2003; Franks 2004); the other RCT had low risk of bias (Iglesias 2004). One RCT recruited groups which may not have been comparable at baseline (Scriven 1998); the Cox regression performed on the IPD took account of between-group differences in prognosis.

\subsubsection{LB compared with compression systems having a paste bandage as the base (five RCTs)}

Five trials were identified for this comparison (Duby 1993; Colgan 1995; Knight 1996; Meyer 2003; Polignano 2004a). In all studies, the 4LB consisted of orthopaedic wool, a crepe bandage, an elastic bandage and an elastic cohesive bandage as the final retaining component. Three trials used a proprietary 4LB kit (Profore) (Colgan 1995; Knight 1996; Polignano 2004a). The paste-bandage system (sometimes referred to as Unna's Boot) varied between trials, consisting of: paste bandage applied over a foam primary dressing with no other compression components (Knight 1996); a two-component system with an elastic cohesive bandage applied after the paste bandage (Polignano 2004a); three-component systems comprising either a paste, a crepe, and an elastic tubular bandage (Duby 1993), or a paste, an elastic, and an elastic tubular bandage (Meyer 2003); and finally, a four-component system consisting of a paste, a crepe and an elastic-cohesive bandage and a class II compression sock (Colgan 1995).

Four RCTs had unclear risk of bias (Duby 1993; Knight 1996; Meyer 2003; Polignano 2004a), and one was at high risk of bias (Colgan 1995).

Pooled data from two trials (71 participants/limbs) that reported complete healing at three months (fixed-effect model) suggested no significant difference between groups: RR $1.34(95 \% \mathrm{Cl} 0.78$ to
2.28), $P$ value 0.29 (test for heterogeneity $P$ value $0.11, I^{2}=60 \%$ ) (Analysis 13.1) (Duby 1993; Colgan 1995). The estimate generated from a random-effects model was similar: RR $1.23(95 \% \mathrm{Cl} 0.54$ to 2.82) (Analysis 13.2). Likewise, the observed between-group difference for complete healing at other time points did not suggest a statistically significant difference: RR 1.13 (0.82 to 1.57$)$ at six months, 68 participants (Polignano 2004a) (Analysis 13.1); and RR 0.82 (95\% Cl 0.66 to 1.01 ) at one year (133 participants) (Meyer 2003) (Analysis 13.1).

Survival analysis was undertaken for two trials (Meyer 2003; Polignano 2004a). One suggested a shorter time to healing with paste bandage (median values 12 versus 16 weeks, $P$ value 0.04 ), with the difference in probability of healing becoming significant after 20 weeks post-randomisation (P value 0.036) (Meyer 2003). The second trial estimated similar values for median days to healing for each study arm (53 for the 4LB and 56 for the paste bandage) (Polignano 2004a). This trial also presented a HR estimate that suggested no significant difference between groups: 1.62 (95\% Cl 0.87 to 3.02$)$, $P$ value 0.13

Two trials reported rate of healing as follows: percentage daily healing rate (Polignano 2004a); and the absolute rate in $\mathrm{cm}^{2}$ per week (Knight 1996). These data were pooled using standardised mean difference (SMD), and suggested a significant treatment effect in favour of the 4LB: SMD 0.52 ( $95 \% \mathrm{Cl} 0.06$ to 0.97 ), P value 0.03 (test for heterogeneity $\mathrm{P}$ value $0.47, \mathrm{I}^{2}=0 \%$ ) (Analysis 13.4).

One RCT reported recurrence, stating that there were no cases of ulcer recurrence during a six month follow-up period (starting from the end of the 12 week treatment period) (Colgan 1995). The same RCT presented costs of bandages per patient over the 12-week trial period (nursing time not included) (Colgan 1995). The estimates for average values (price year not stated) were IEP 82.54 for the 4LB and IEP 66.24 for the paste bandage (statistical tests not reported). Another RCT found no significant difference between groups in change in patient-reported pain score assessed by visual analogue scale from baseline to final assessment ( $P$ value 0.32 ) (Polignano 2004a). Three trials reported withdrawal rates and observed similar rates for both study groups, with a small number due to adverse events (Colgan 1995; Meyer 2003; Polignano 2004a).

\section{Summary of evidence for Section 2.4.3 Comparison between the} 4LB and compression systems with a paste bandage as the base

No significant differences were found between the 4LB and paste bandages for complete healing at three months (Duby 1993; Colgan 1995), six months (Polignano 2004a), and one year (Meyer 2003). Estimates of time to healing showed no difference between groups in one trial (Polignano 2004a), and a significant difference in favour of the paste-bandage system in another (Meyer 2003). This difference in outcome could be explained by variation in the components of the paste-bandage systems, two components being used in one trial (Polignano 2004a), and three components in the other (Meyer 2003); different systems could exert different amounts of compression. Different RCTs individually assessed different secondary outcomes, finding no between-group differences for recurrence, pain, withdrawals and adverse effects. Findings from one RCT suggested lower costs for the paste-bandage system (Colgan 1995). All RCTs had unclear risk of bias apart from Colgan 1995 which had high risk of bias. 
2.5 Adjustable compression boots compared with compression bandages (two RCTs)

Two small trials were identified for this comparison (DePalma 1999; Blecken 2005). Both studies described the adjustable boot as an inelastic compression garment, and both evaluated different versions of the CircAid proprietary device. One had a high risk of bias (DePalma 1999), while risk of bias for the other was unclear (Blecken 2005).

\subsubsection{Adjustable compression boots compared with paste bandages}

The first trial (38 participants) evaluated an adjustable compression boot consisting of a series of interlocking, non-elastic bands that encircled the leg, held in place by hook and loop fasteners, together with a foot-piece made of very low stretch bands (DePalma 1999). Participants were instructed to adjust the straps in order to maintain compression. This was compared with a paste-bandage system. Three different estimates of healing rate were reported: mean area healed $\left(\mathrm{cm}^{2}\right)$ per day; mean area healed (\%) per day; and the linear healing rate of the wound edge towards the wound centre (mean $\mathrm{cm}$ per day). No statistically significant differences were observed for any of these outcomes (Analysis 14.1). The mean total cost per patient completing the trial based on costs of clinician time and materials suggested a lower cost for patients receiving the adjustable compression boot: USD 559.41 versus USD 901.73, $P$ value 0.05 (price year not stated). Two participants withdrew from the group receiving the adjustable boot, and five withdrew from the paste bandage group.

\subsubsection{Adjustable compression boots compared with the 4LB}

The second study entailed within-individual randomisation whereby 12 participants with bilateral venous leg ulcers were recruited (Blecken 2005). One limb per participant was randomised to receive the adjustable compression boot, similar to the device used in the above study, except that the adjustable bands were made of Velcro. Application of the boot was preceded by a paraffinimpregnated gauze primary dressing, sterile absorbent gauze, and a felt pad cushion, all retained with a cotton stockinette. An elastic anklet was applied over the boot. The second limb was allocated a four-component compression system; this differed from the traditional $4 \mathrm{LB}$, consisting of paraffin-impregnated gauze primary dressing, sterile absorbent gauze, felt pad, gauze bandage and elastic bandage. At 12 weeks, four limbs out of 12 healed in each group, these limbs belonging to the same participants in each group. There were no withdrawals.

\section{Summary of evidence from Section 2.5: Adjustable compression boots compared with compression bandages}

Two RCTs found no evidence of a difference in healing between adjustable compression boots and compression bandage systems. Both trials were small with high (DePalma 1999) and unclear (Blecken 2005) risk of bias. The compression boot may be a less costly option (DePalma 1999).

\subsection{Compression stockings or tubular devices compared with compression bandage systems (11 RCTs)}

Eight trials compared compression stockings with compression bandages (Hendricks 1985; Koksal 2003; Jünger 2004b; Polignano 2004b; Mariani 2008; Taradaj 2009; Brizzio 2010; Szewczyk
2010), and three compared tubular compression devices with compression bandages (Jünger 2004a; Milic 2007; Milic 2010).

Compression stockings are usually garments which are fashioned to the shape of the lower leg, with open or closed, tailored toes and heels. Different sizes are available to correspond to varying limb sizes. They are designed to provide graduated compression, with higher pressures exerted at the ankle, diminishing up to the top of the calf. Tubular compression devices are usually available as lengths of close weave stretch cotton, open at the toe and sometimes also at the heel; they can provide either uniform or graduated compression, depending on device specifications. Again, different sizes are available to fit different size limbs.

Compression stockings were compared with paste bandages (Hendricks 1985; Koksal 2003), the SSB (Jünger 2004b; Polignano 2004b; Mariani 2008; Taradaj 2009; Brizzio 2010), a two-component bandage system (Szewczyk 2010), and the 4LB (Szewczyk 2010). In the evaluations of tubular compression, the comparators were the SSB (Jünger 2004a), elastic bandages (Milic 2007), and elastic bandages and tubular compression combined (Milic 2010).

\subsubsection{Compression stockings compared with paste bandages (two RCTs)}

Two trials compared a single-layer compression stocking with a paste bandage: Hendricks 1985 which had unclear risk of bias; and Koksal 2003, which was classified as being at high risk of bias. In the Hendricks 1985 trial (21 participants), the device was designed to provide graduated compression from $24 \mathrm{~mm} \mathrm{Hg}$ at the ankle to $16 \mathrm{~mm} \mathrm{Hg}$ at the calf. In the Koksal 2003 trial (60 participants), the stocking was designed to provide 30 to $40 \mathrm{~mm} \mathrm{Hg}$ compression.

No significant differences were detected between groups in terms of complete healing at four months, RR $1.05(95 \% \mathrm{Cl} 0.74$ to 1.48) (Koksal 2003), or 18 months, RR $0.91(95 \% \mathrm{Cl} 0.64$ to 1.29) (Hendricks 1985) (Analysis 15.1). One trial report included presentation of raw data, allowing the review authors to calculate estimates from Kaplan-Meier survival analysis (Hendricks 1985). The estimated cumulative proportions healed at 18 months were $73 \%$ for participants randomised to the stocking and $90 \%$ for those allocated to the paste-bandage system. The estimates for median time to healing were 18 weeks versus seven weeks respectively ( $P$ value 0.39 , log rank test). The other trial reported no significant difference between groups for mean weeks to healing: 6.65 for stocking and 6.85 for the paste bandage ( $P$ value $>0.05$ ) (Koksal 2003). In terms of secondary outcomes, one trial demonstrated significantly lower mean pain scores for participants allocated the stocking (P value $<0.0001$ ) (Koksal 2003). One treatment-related adverse event was reported in the group receiving the stocking, but the nature of this was not described. Four participants allocated the stocking withdrew compared with three from the paste bandage group, one of these having a severe reaction to the dressing. In the Hendricks 1985 trial, there were no withdrawals from the paste bandage group and one from the stocking group; the reason was not described.

\subsubsection{Compression stockings compared with inelastic bandages} (the SSB) (five RCTs)

2.6.2.1 Compression stockings (low compression) compared with inelastic bandages (the SSB) 
One trial (60 participants) compared a single-layer compression stocking designed to exert 15 to $20 \mathrm{mmHg}$ at the ankle with an inelastic bandage system (three short-stretch slings), both devices being left in place day and night (Brizzio 2010). This trial was classified as being at high risk of bias.

No significant difference was detected between treatment groups in terms of complete healing at three months RR $0.67(95 \% \mathrm{Cl}$ 0.35 to 1.29$)$ (Analysis 16.1), or six months RR $0.68(95 \% \mathrm{Cl} 0.42$ to 1.10 ) (Analysis 16.2). Median days to healing were similar in both groups ( 56 days for the group receiving stockings and 60 days for SSB). Analyses of quality of life scores (using the Chronic Venous Insufficiency Quality of Life questionnaire) did not detect any statistically significant between group differences at 35 days, or at final assessment in participants with healed ulcers. Four participants withdrew from the group allocated the stocking (one death, one did not attend clinic and two had systemic infections), whilst one withdrew from the group allocated SSB because his/her condition deteriorated rapidly.
2.6.2.2 Compression stockings (high compression) compared with inelastic bandages (the SSB)

Four trials compared high compression stockings with the SSB (Jünger 2004b; Polignano 2004b; Mariani 2008; Taradaj 2009). Three were at high risk of bias (Jünger 2004b; Mariani 2008; Taradaj 2009), and one was unclear (Polignano 2004b). One trial reported the mean (standard deviation) ankle pressure for the stocking system as 42.7 (13.0) $\mathrm{mmHg}$ (Jünger 2004b). In the other three trials, the devices were designed to provide 25 to $32 \mathrm{mmHg}$ (Taradaj 2009), 35 $\mathrm{mmHg}$ (Polignano 2004b) and $39 \mathrm{mmHg}$ (Mariani 2008).

Data from all four trials (317 participants) were pooled using a fixed-effect model and showed that significantly more participants achieved complete healing with stockings at two to four months than with the SSB: RR $1.62(95 \% \mathrm{Cl} 1.26$ to 2.10$)$, P value 0.0002 (test for heterogeneity $\mathrm{P}$ value $0.06, \mathrm{I}^{2}=60 \%$ ) (Analysis 17.1, Figure 10) (Jünger 2004b; Polignano 2004b; Mariani 2008; Taradaj 2009). The between-group difference remained statistically significant when the analysis was repeated using a random-effects model: RR 1.66 (95\% Cl 1.07 to 2.58), P value 0.02 (Analysis 17.2).

Figure 10. Forest plot of comparison: 17 Higher compression stockings versus SSB, outcome: 17.1 Patients with complete healing at 2-4 months (fixed-effect).

\begin{tabular}{|c|c|c|c|c|c|c|c|c|c|}
\hline \multirow[b]{2}{*}{ Study or Subgroup } & \multicolumn{2}{|c|}{ Hlgh-compression stocking } & \multicolumn{2}{|c|}{ Short-stretch bandage } & \multirow[b]{2}{*}{ Weight } & \multirow{2}{*}{$\begin{array}{c}\text { Risk Ratio } \\
\text { M-H, Fixed, 95\% Cl }\end{array}$} & \multirow{2}{*}{\multicolumn{2}{|c|}{$\begin{array}{c}\text { Risk Ratio } \\
\text { M-H, Fixed, 95\% Cl }\end{array}$}} & \\
\hline & Events & Total & Events & Total & & & & & \\
\hline Jünger 2004b & 29 & 61 & 19 & 60 & $38.3 \%$ & $1.50[0.95,2.37]$ & & & \\
\hline Mariani 2008 & 25 & 30 & 21 & 30 & $42.0 \%$ & $1.19[0.90,1.58]$ & & & \\
\hline Polignano 2004b & 12 & 27 & 5 & 29 & $9.6 \%$ & $2.58[1.05,6.35]$ & & & \\
\hline Taradaj 2009 & 15 & 40 & 5 & 40 & $10.0 \%$ & $3.00[1.20,7.47]$ & & & \\
\hline Total $(95 \% \mathrm{Cl})$ & & 158 & & 159 & $100.0 \%$ & $1.62[1.26,2.10]$ & & & \\
\hline Total events & 81 & & 50 & & & & & & \\
\hline $\begin{array}{l}\text { Heterogeneity: Chi } \\
\text { Test for overall effec }\end{array}$ & $\begin{array}{l}7.47, d f=3(P= \\
Z=3.70(P=0.0\end{array}$ & $60 \%$ & & & & & $\begin{array}{|ccc|}0.1 & 0.2 & 0.5 \\
& \text { Favours SSB }\end{array}$ & 12 & $\begin{array}{l}510 \\
\text { tockings }\end{array}$ \\
\hline
\end{tabular}

Three of the above trials reported outcomes relating to time to healing (Jünger 2004b; Polignano 2004b; Mariani 2008). In Jünger $2004 \mathrm{~b}$ the cumulative proportions healed at three months (as read from a plot of survival curves) were $51 \%$ for the group receiving stockings and $30 \%$ for the SSB (P value 0.057 , log rank test). The same trial reported no significant difference between groups for median (range) days to healing: 47 (10 to 83 ) for stockings versus 52 (6 to 80) for SSB (P value 0.82, Mann-Whitney U-test). The other two trials reported the less useful mean time to healing: 72 days for stockings versus 101 days for SSB ( $P$ value 0.027 , log rank test) (Polignano 2004b), and 56 versus 61 days respectively (P value 0.52) (Mariani 2008).

Three of the four trials reported a variety of secondary outcomes (Jünger 2004b; Polignano 2004b; Mariani 2008). In Polignano 2004b, both decrease in ulcer pain during the trial and comfort while wearing compression were significantly better for the group receiving stockings ( $P$ value 0.017 and $P$ value 0.038 , respectively). A higher withdrawal rate was noted in the SSB group in this trial (38\% versus $15 \%)$. Of five participants withdrawing from the SSB group, one was considered to be related to compression (bullous dermatitis); there were no reported withdrawals due to adverse events in the group receiving stockings. The Jünger $2004 \mathrm{~b}$ trial reported 29 adverse events (two serious) in 20 of the $65(31 \%)$ participants receiving stockings and 42 adverse events (four serious) in 26 of the 67 (39\%) participants receiving the
SSB. Withdrawal rates were similar between groups. This trial also estimated cost of procedures including labour and associated resources. The estimated mean cost per percentage reduction in wound area (price year 2003) was EUR 2.57 for the group receiving stockings and EUR 4.58 for the SSB group. The Mariani 2008 trial reported significantly worse performance for the SSB for inhibition of activities ( $P$ value 0.025 ), pain at donning and removal of device ( $P$ value 0.001 ), and number of problems reported in terms of pain, discomfort and hindrance of activities ( $P$ value $<0.0001)$. No significant differences, however, were detected between groups for daytime pain and discomfort. There were four withdrawals from the group receiving stockings (one withdrew consent and three had difficulty putting on the stockings) and none among those allocated the SSB.

\subsubsection{Compression stockings compared with multi-component bandage systems (one RCT)}

A three-armed trial (46 participants) at unclear risk of bias compared a compression stocking with a two-component compression bandage system and the 4LB (Szewczyk 2010). The comparison between the two types of bandage was considered earlier (Section 2.2.2). The stocking was described as Class II, presumably designed to deliver pressure of 18 to $24 \mathrm{mmHg}$, although this was not specified in the trial report, which also did not describe characteristics such as the number of layers. No details 
were provided about whether the devices were worn both day and night.

No statistically significant between-group difference was detected for complete healing at three months for either comparison: RR 0.85 $(95 \% \mathrm{Cl} 0.47$ to 1.57$)$ for the stocking versus the two-component bandage system (Analysis 18.1); and RR 0.89 (95\% Cl 0.47 to 1.67 ) for stockings compared with 4LB (Analysis 19.1). No secondary outcomes were reported.

\subsubsection{Tubular compression compared with inelastic bandages (the SSB) (one RCT)}

A heel-less, open-toed, graduated, tubular compression device providing 30 to $40 \mathrm{mmHg}$ at the ankle was compared with an inelastic bandage (the SSB) in a non-inferiority trial (178 participants) (Jünger 2004a). This RCT was classified as being at high risk of bias.

There was no statistically significant difference between groups for complete healing at three months: RR $0.98(95 \% \mathrm{Cl} 0.76$ to 1.26), $P$ value 0.86 (Analysis 20.1). Kaplan-Meier analysis suggested a median estimate of 42 days to healing in both groups and found no significant difference in probability of healing derived from survival curves ( $P$ value 0.41 ). In terms of adverse events, $14 \%$ of participants receiving tubular compression complained of pain or tightness during treatment which was resolved in all cases by using a larger-sized device; the participants receiving the SSB did not experience such problems, and no other adverse events were reported. Assessment of health-related quality of life using the Nottingham Health Profile suggested no difference between treatment groups.

\subsubsection{Tubular compression compared with, or added to, elastic bandages (two RCTs)}

Two trials from the same research team evaluated tubular compression systems: Milic 2007 was classified as being at high risk of bias; and Milic 2010 at being at unclear risk of bias. The first trial compared two four-component systems consisting of gauze bandage, crepe bandage, a graduated tubular device and an elastic bandage versus gauze bandage, crepe bandage and two elastic bandages (Milic 2007). The second trial included three arms and compared: gauze bandage, crepe bandage and a tubular device; gauze bandage, crepe bandage, a tubular device and an elastic bandage; gauze bandage, crepe bandage, a tubular device and two elastic bandages (Milic 2010). In both trials, the tubular devices provided an ankle pressure of 30 to $40 \mathrm{mmHg}$; all participants wore compression day and night and received mechanical debridement using sterile gauze, a dry dressing for non-exuding wounds and application of boric acid in cases of exudation. At first scrutiny the participant populations from these two RCTs appear very similar; the trial authors confirmed that the two RCTs are entirely separate (personal communication).

In the first trial (150 participants) the cumulative proportions of participants healed at 16 months were 93\% for tubular compression and $51 \%$ for the system including two elastic bandages ( $P$ value $<0.01$ ) (Milic 2007). This trial also reported shorter median days to healing for the group receiving tubular compression: 133 (range 28 to 464) versus 211 (range 61 to 438), $P$ value not reported. There were significantly lower recurrence rates at one year for the group receiving tubular compression $(24 \%$ versus $53 \%$, P value $<0.05)$. Amongst the participants who received tubular compression, $17 \%$ experienced skin excoriation of the ankle or leg and $47 \%$ experienced pressure or pain caused by slippage of the device; details of adverse events were not provided for the group that received compression bandages. There were fewer withdrawals in the tubular system group (4\% versus $12 \%$ ), with the majority of withdrawals (8/9 participants) from the bandage group being due to participants requesting change to the alternative treatment (Milic 2007).

In the second trial (131 participants) that evaluated the effects of adding elastic bandages to a base system of gauze bandage, crepe bandage and tubular compression (Milic 2010), the number of participants experiencing complete healing at six months was significantly greater for the addition of both one and two elastic bandages. The respective RR estimates were $0.46(95 \% \mathrm{Cl} 0.28$ to $0.75)$, $P$ value 0.002 (88 participants) (Analysis 21.1$)$, and 0.42 ( $95 \%$ $\mathrm{Cl} 0.26$ to 0.68 ), $\mathrm{P}$ value 0.0004 (85 participants) (Analysis 22.1). No significant difference, however, was detected between the addition of one versus two elastic bandages: RR 0.91 ( $95 \% \mathrm{Cl} 0.69$ to 1.18), $\mathrm{P}$ value 0.47 (89 participants) (Analysis 23.1). Median weeks to healing were similar in all three groups: 12 weeks (range 5 to 24) in the group receiving the base system; 11 weeks (range 3 to 25) with one elastic bandage added; and 14 weeks ( 5 to 24 ) for two additional elastic bandages. The trial authors reported that estimates from adjusted Cox regression indicated significantly higher probability of healing among participants receiving two added elastic bandages when compared with the other two groups ( $P$ value $<0.001$ versus the base system, and $P$ value 0.017 versus one elastic bandage). Eleven participants withdrew from the trial: one from the group receiving the base system, one from the group receiving one additional elastic bandage ( $2 \%$ of participants in both cases), and nine (21\%) from the group allocated two elastic bandages.

\section{Summary of evidence from Section 2.6: Compression stockings or tubular devices compared with compression bandage systems}

All RCTs were at high or unclear risk of bias. When compared with the SSB, use of a high compression stocking was associated with: better healing at up to four months (four RCTs pooled) (Jünger 2004b; Polignano 2004b; Mariani 2008; Taradaj 2009); better outcomes for some aspects of pain/discomfort (Jünger 2004b; Mariani 2008); and lower costs (Jünger 2004b).

Healing outcomes were better at 16 months for participants receiving a four-component system that included a tubular device plus elastic bandage when compared with four components that included two elastic bandages; there were fewer withdrawals and a lower rate of ulcer recurrence at one year for the tubular device, however, more adverse events were reported in this group (Milic 2007). When one or two elastic bandages were added to a base three-component system that included an outer tubular layer, healing outcomes were better for the two groups receiving elastic bandages. There was no significant difference in healing between the two elastic bandage groups. There were more withdrawals in the group receiving two elastic bandages relative to the other two groups (Milic 2010). When a tubular compression system was compared with the SSB no differences in healing or quality of life outcomes were detected at three months; however, adverse events were more frequent in the tubular compression group (Jünger 2004a).

No significant between-group differences were found for the following comparisons (all small RCTs): single-layer stockings and 
paste bandages for complete healing at four months (Koksal 2003), and at 18 months (Hendricks 1985); low compression stocking and SSB for healing at three or six months, nor in quality of life outcomes (Brizzio 2010); stockings and two-component bandages for healing at three months (Szewczyk 2010); stockings and the 4LB for healing at three months (Szewczyk 2010).

\section{DISCUSSION}

\section{Summary of main results}

The evidence suggests that venous ulcers heal more rapidly with compression than without, and that multi-component bandage systems achieve better healing outcomes than single-component bandages. When competing systems comprising two-component bandages were compared, there was some evidence to suggest that those that included an elastic component might be more effective; a similar finding was noted for alternative three-component bandage systems. No differences were observed in terms of healing between a two-component bandage system and the 4LB, nor between different variations of the original 4LB. Estimates from survival analyses of IPD indicated faster healing for the 4LB compared with the SSB. No differences were observed between the $4 \mathrm{LB}$ and paste-bandage systems, but interpretation could have been hampered by differential performance of variants of the paste bandages. There was no difference in healing outcomes between the adjustable compression boot and compression bandages, nor between a single-layer stocking and a paste-bandage system, however, overall the evidence was not of high quality and had low statistical power for detection of clinically important differences. When high-compression stocking systems were compared with the SSB, healing outcomes were in favour of the stockings, but there was no difference seen between low compression stockings and the SSB. One small trial compared stockings with two-component bandages and the 4LB and found no between-group differences between stockings and bandages. A large ongoing trial comparing compression stockings with the 4LB will inform this comparison further (Dumville 2009). Less pain was observed for all types of stockings when compared with bandages. There was no difference between tubular compression and the SSB for healing. Better healing outcomes were achieved when elastic bandages were added to tubular compression. In terms of cost-effectiveness, most evaluations were of costs only. One trial conducted a rigorous costeffectiveness analysis in which the 4LB emerged as the dominant treatment strategy when compared with the SSB.

There was limited evidence on ulcer recurrence, with three trials reporting this outcome (Colgan 1995; Morrell 1998; Milic 2007). One reported no cases of recurrent ulceration in participants receiving single-component elastic compression, the $4 \mathrm{LB}$ or fourcomponent compression comprising a paste bandage when rates were assessed during a six-month follow-up period following 12 weeks of treatment (Colgan 1995). Another trial that compared application of the $4 \mathrm{LB}$ in the context of a specialist clinic with usual care by the district nurse did not detect a statistically significant difference between groups for recurrence rates, or time to recurrence, during the one-year trial period (Morrell 1998). In an evaluation of tubular compression versus compression bandages, significantly lower recurrence rates were detected in the group receiving tubular compression at one year (Milic 2007). It is likely that the majority of included trials lacked the statistical power and duration of follow-up required to detect meaningful recurrence rates following treatment with compression therapy.
This review has attempted to take account of recent recommendations concerning the classification and description of different systems of compression (Partsch 2008b). This update refers to the numbers of components in compression systems rather than the number of layers, as it has been argued that the number of components is more meaningful.

\section{Overall completeness and applicability of evidence}

\section{Co-interventions, confounding factors and external validity}

The study selection criteria stipulated that the bandages being studied should be the only systematic difference between treatment groups. In practice, this criterion has been difficult to apply without excluding many trials of important types of compression therapy from the review. One example of this is where a specialised package of care incorporating multi-component compression is compared with usual care that does not routinely include compression (Charles 1991; Taylor 1998; Morrell 1998; O'Brien 2003). In these trials, application of the bandage is not the only difference between treatment arms since the characteristics of care providers vary between groups with compression in one arm being provided by staff with specialist training and experience who could advise patients more generally about the management of their venous leg ulcer, for example, regarding limb elevation and mobility. The evaluations of paste bandages and Unna's boot also introduce an additional, non-bandage difference between groups (for example Duby 1993; Colgan 1995; Knight 1996; Meyer 2003; Polignano 2004a). These devices normally provide a primary wound contact layer as well as compression bandaging. The alternative study arm is likely to receive a different type of primary dressing (e.g. foam dressing or hydrocolloid) prior to application of bandages or stockings. Factors such as additional aspects of care used together with compression, or different primary dressings between treatment arms may obscure the treatment effect due to the compression, and so hinder the interpretation of findings.

In many of the included trials, the observed treatment effect may have been influenced further by imbalance of treatment groups at baseline with respect to independent prognostic factors. The literature on healing prognosis has shown consistently that baseline ulcer area and ulcer duration are significant independent predictors of delayed healing (Skene 1992; Franks 1995; Margolis 2000; Margolis 2004; Brown 2004). Some of the more recent trials addressed this by using stratified randomisation and undertaking analyses that adjusted for covariates, such as Cox proportional hazards models (for example Franks 2004; Iglesias 2004; Brizzio 2010; Milic 2010), or logistic regression (Brizzio 2010). When such methods are not used, and particularly when trials are small in size, the estimated treatment effect may be prone to bias because of chance differences in the baseline prognostic profiles of treatment groups.

Several trial reports stated that venous leg ulcers above certain dimensions would not be eligible for inclusion, for example, maximum eligible baseline ulcer surface area $15 \mathrm{~cm}^{2}$ (Szewczyk 2010), and $50 \mathrm{~cm}^{2}$ (Cordts 1992; Brizzio 2010). Six RCTs with this type of restriction reported comparisons involving compression stockings or tubular devices (Jünger 2004a; Jünger 2004b; Polignano 2004b; Mariani 2008; Brizzio 2010; Szewczyk 2010); other comparisons were alternative single-component bandages (Cordts 1992), and three-component bandage systems (Meyer 2002), also paste bandages compared with an adjustable compression boot 
(DePalma 1999) and the four-layer bandage (Meyer 2003). Patients with much larger wounds may present in clinical practice and so some findings may have limited external validity.

\section{Quality of bandage application}

It has been suggested that the clinical effect of compression is partly dependent on the skill of the bandager in achieving the correct amount of sub-bandage pressure and a pressure graduated from toe to knee (Feben 2003). Findings from an online survey of 304 clinicians in the UK, USA and Germany suggested that challenges in the use of compression included difficulty in obtaining precise pressures; also, the need for training in application of compression bandages was highlighted. The survey authors also observed variation in the use of different types of compression systems across the countries studied (Cullen 2009). It is possible that the performance of certain compression systems may be enhanced because of greater staff familiarity and experience in that setting. The differential effects of 4 LB and SSB seen in the IPD meta-analysis could be partly explained by skill and experience of bandagers; three out of the five included trials (representing $75 \%$ of included participants) were based in the UK, where the 4LB is standard (Scriven 1998; Franks 2004; Iglesias 2004), whilst the others were based in continental Europe, where the SSB is standard treatment (Partsch 2001; Ukat 2003). Information regarding bandager skill was not collected during these trials, and so the effect of this variable could not be investigated further. As far as we could ascertain, the methods used for application of both types of bandage were in line with manufacturers' recommendations, relevant clinical guidelines, and expert guidance. It would be useful if future trials could collect data on staff skills at baseline, and this information could be included as a covariate in the modelling of treatment effect.

It is also difficult to estimate what influence staff skills may have had on the observed estimates of treatment effect for other comparisons in this review. Some trials indicate a possible move towards compression systems that are less dependent on practitioner skill, where patients and their relatives may contribute more to application of devices, namely compression stockings (Hendricks 1985; Jünger 2004b; Polignano 2004b; Mariani 2008).

A related aspect which may influence the effectiveness of treatment is patient concordance with compression regimens. A mixed methods study combining semi-structured interviews with clinicians with an online survey found that patient concordance with compression is a major concern amongst care providers (Cullen 2009). A literature review of studies evaluating patient concordance with compression therapy for ulcer healing reported rates of non-concordance from observational studies in the range of $10 \%$ to $80 \%$ and also suggested that healing was delayed in patients defined as having lower levels of concordance. It should be noted that these findings were based on a small number of studies of uncertain methodological quality (Moffatt 2009).

\section{Quality of the evidence}

The methodological quality of evidence in this field is variable. All RCTs except one were classified as being at high or unclear overall risk of bias. A general observation is that quality appears to be improving over time, with trials published within the last ten years more likely to have taken important steps to reduce bias in estimates of treatment effect by using proper methods of randomisation (i.e. unpredictable allocation to treatment groups), allocation concealment, blinded outcome assessment and performing analysis by intention to treat. More recent trials are more likely to be larger and to have been based on prior estimation of the required sample size to detect a defined difference in outcome between groups. Interpretation of older trials is often difficult because of small sample sizes and problems with methodological quality. Furthermore small trials are more likely to result in chance estimates of treatment effect because of imbalances between treatment groups for prognostic factors such as ulcer surface area or duration. The possible impact of such baseline imbalances is usually difficult to interpret post hoc, and ideally would be adjusted for in the primary analysis (an approach more commonly taken in more recent, high-quality studies). Shortcomings in the statistical analysis of trial data were frequently encountered. Some studies report the mean (rather than median) time to healing which could result in biased estimates as such analysis is based on all participants having healed and/or the survival curve having an assumed shape (the shape is not assumed in non-parametric survival analysis). For continuous outcomes such as healing rate and change in ulcer area, data are likely to be skewed but transformation is usually not mentioned. Again, this could have influenced the derived estimates of effect (Bland 2000). More recent trials include survival analyses for time to healing (for example Scriven 1998; Franks 2004; Iglesias 2004; Jünger 2004a; Jünger 2004b; Nelson 2007a; Brizzio 2010; Milic 2010), and this provides a more meaningful estimate of treatment effect, particularly if HR estimates are provided, adjusted for prognostic factors. All future trials should incorporate such analyses.

Frequently interventions are not described comprehensively in trial reports, so it can be difficult to judge the degree of compression being applied, and impossible for readers to apply the evidence directly. A typical example of this is the term "Unna's boot" which is used to characterise the compression system, but is often not described further. It is clear from studying the trials included in this review that the definition of Unna's boot varies, and there does not appear to be an agreed definition in the literature. The basis for this type of compression is a paste-impregnated bandage (usually zinc oxide and calamine) (Kikta 1988), and in some cases this is the sole component. However, the paste bandage can also be applied as part of a multi-component system comprising two, three or four components, all of which could perform differently. Trial reports should include details of the number and type of components, the materials used, the dimensions of bandages and the technique of application (e.g. spiral, figure-of-eight), as recommended by an expert consensus group (Rabe 2008).

\section{Potential biases in the review process}

Although the search strategy was comprehensive, it is possible that eligible unpublished trials could remain unidentified. One unpublished trial of compression came to light during a wound management conference (Nelson 2007b). Communication with the trial authors confirmed that: the trial was small (40 participants), compared the $4 \mathrm{LB}$ with SSB, and had terminated prematurely (personal communication, Professor Charles McCollum). Since no baseline or outcome data were available, it is not possible to judge the potential effect of including this trial in the review. Other eligible unpublished evaluations may exist that have not been identified by the review process. Therefore, the effect of publication bias on this review should not be discounted. 


\section{Agreements and disagreements with other studies or reviews}

This updated review includes new evidence concerning competing multi-component bandage systems (two-component bandage versus the 4LB) as well as comparisons between compression bandages and stockings. In addition, a rigorously conducted and informative IPD meta-analysis of the comparison between four-layer and SSBs has been incorporated into the review. The findings of the previous version of this review are largely upheld, and this update provides some additional evidence to indicate that multi-component systems that include an elastic bandage are more effective in terms of wound healing compared with multi-component systems with inelastic constituents. This finding conflicts with some aspects of consensus-based recommendations of compression classification, where it was suggested that multicomponent systems that include elastic constituents will perform similarly to inelastic systems overall because of friction between different elements (Partsch 2008b). The findings of this review suggest that compression stockings may perform better than the SSB in terms of wound healing; however, data are still lacking on the important comparison of compression stockings versus the 4LB. A large RCT addressing this comparison is ongoing (Dumville 2009).

Updates of this review, in contrast with the parent review, have included only those trials where treatment allocation was described as random. Consequently two studies that were included in the original version of the review are now excluded. The comparisons involved were: compression (Unna's Boot) versus no compression (dressing alone) (Sikes 1985); and compression stockings versus SSB (Horakova 1994). A third trial that evaluated two different three-component systems was excluded because we became aware that participants in one treatment arm also received steroids (Northeast 1990).

We identified two other systematic reviews of compression therapy for venous leg ulceration (Palfreyman 1998; Amsler 2009). The study selection criteria of the Palfreyman review differed from this review in that quasi-randomised studies, evaluations of intermittent pneumatic compression and those with recurrence as the primary outcome were included, whereas studies where venous disease was not confirmed by vascular assessment were excluded. In addition, the literature search was restricted to English language articles. This resulted in eight trials being identified as eligible for inclusion and these were sub-grouped for metaanalysis purposes according to the type of compression evaluated. Some findings reflected those of the current review in terms of healing: multi-component compression was more effective than single-component systems (based on Nelson 1995, a secondary reference to Nelson 2007a in this Cochrane review); and multicomponent compression comprising an elastic bandage performed better than that consisting of non-elastic devices (based on Callam 1992b, included as a primary reference in this review). However, the Palfreyman review included only one trial in the comparison of compression versus no compression (Kikta 1988), estimating no statistically significant difference between groups. Two further studies were described as comparing Unna's Boot with 'other therapies', whereas in the current review these were included within the following comparisons: compression versus no compression (non-compressive bandages) (Rubin 1990); and competing single-component compression systems (Cordts 1992). Of the remaining studies included in the Palfreyman review, one focused on ulcer recurrence and the other two evaluated the effectiveness of intermittent pneumatic compression. We took the view that it was more helpful to include studies not reporting vascular assessment of venous disease since methods of diagnosis vary between studies and are also likely to vary in clinical practice, meaning that a standardised definition may not be realistic.

The second review included comparisons of compression bandages with compression stockings in patients with venous leg ulcers (Amsler 2009). This review had some systematic elements but did not include a structured assessment of risk of bias and did not mention checking of study selection decisions and data extraction by a second, independent reviewer. Eight trials were included, seven of which were also included in our review. We excluded the eighth trial because it used quasi-randomisation (Horakova 1994); Amsler and colleagues also expressed concern about the quality of randomisation for this trial. The reviewers confidently concluded that stockings were significantly better than bandages in terms of complete healing at 12 to 16 weeks, time to healing and pain scores. However, there were problems with the analyses underpinning these conclusions. The meta-analysis of complete healing pooled all eight included studies, despite substantial clinical heterogeneity in terms of the types of stockings and bandages used across the group of trials. Time to healing was analysed as a continuous outcome and survival analyses were not considered. The metaanalysis of pain scores was based on three trials, and showed significant statistical heterogeneity. We feel that slightly more cautious conclusions are warranted, given the methodological quality and clinical heterogeneity of this group of trials.

\section{AUTHORS' CONCLUSIONS}

\section{Implications for practice}

Compression increases the healing rates of venous leg ulcers compared with no compression. Multi-component compression systems are more effective than single-component systems. Multicomponent systems containing an elastic bandage appear to be more effective than those composed mainly of inelastic constituents. Two-component bandage systems appear equivalent to the four-layer bandage (4LB) in terms of healing. Variations of the 4LB achieve similar outcomes. The 4LB heals ulcers faster and is more cost-effective than multi-component systems comprising a short stretch bandage (SSB). There does not appear to be any difference between the $4 \mathrm{LB}$ and paste-bandage systems, but interpretation of data is impaired by differences in the paste-bandage systems. There is currently no evidence of a difference in the effectiveness of adjustable compression boots and compression bandage systems, or between single-layer stockings and paste-bandage systems. Two-layer stockings appear to be more effective than the SSB. The relative effectiveness of compression stockings and the $4 \mathrm{LB}$ is currently unclear. The relative effectiveness of tubular compression and compression bandages is currently unclear. The limited evidence on the effects of different compression systems on venous ulcer recurrence precludes definitive conclusions at the current time. The performance of any type of compression bandage might be influenced by operator skill; this is likely to be less of an issue for compression stockings.

\section{Implications for research}

Some of the research concerning management of venous leg ulceration is of poor quality, but methodological improvements are 
seen in more recent trials, possibly as a result of the CONSORT Statement, a document that provides guidance regarding the reporting of randomised controlled trials (Schulz 2010). The following are recommended for future studies:

- Recommendations outlined in the CONSORT Statement should be adopted as far as possible.

- If possible, future trials should be conducted in collaboration with a clinical trials unit in order to provide the optimal infrastructure for trial design, conduct, data management and analysis.

- Recruitment numbers should be based on an a priori sample size calculation. In many trials the sample size is too small to detect clinically important differences between treatments as statistically significant. In order to recruit sufficient patient numbers, multicentred trials should be considered more frequently. When these trials are commissioned, a strong infrastructure will be required to provide support and promote collaboration.

- A proper method of randomisation should be used and reported (e.g. computer-generated list), and allocation to treatment should be concealed (e.g. using remote telephone randomisation service).

- The primary endpoint of treatment trials should be complete ulcer healing, and, preferably, the primary outcome should be time to healing. Assistance should be sought from a suitably qualified statistician regarding the design and analysis of the trial in relation to survival analysis. In addition, the length of follow-up needs to be of sufficient duration to capture a meaningful proportion of events. If time-to-event analysis is not feasible, other outcomes could include frequency of complete healing during the trial period, or (less preferably) healing rate and change in ulcer surface area.

- A single reference ulcer should be selected for each patient. Multiple ulcers on a patient should not be studied unless the trial has been specifically designed to accommodate this, and appropriate statistical analysis prespecified to account for clustering.

- Treatment groups should be comparable at baseline for prognostic factors such as ulcer area and duration. In small RCTs randomisation alone may not achieve balance for prognostic factors. Statistical analysis should adjust for baseline imbalance.

- A complete and thorough description of concurrent treatments, including primary dressings, should be given in trial reports.

- Assessment of outcomes should be undertaken either by assessors masked to trial treatment, or independently confirmed by assessors masked to treatment.

- Analysis should be according to intention to treat.

- Evaluations should provide sufficiently full details of the interventions used, including descriptions of all components of compression, such that readers would be able to apply the treatments described (with training where necessary).
- Evaluations should report the skill level of staff providing care.

\section{ACK N OWLEDGEMENTS}

The following greatly assisted in the production of the first version of this review: Alison Fletcher and Trevor Sheldon who were coauthors of the original review; Julie Glanville, formerly of the Centre for Reviews and Dissemination, University of York; Mary Bliss, Andrew Boulton, Nick Bosanquet, Richard Bull, Michael Callam, Carol Dealey, Peter Friedman, Brian Gilchrist, Keith Harding, Deborah Hoffman, Vanessa Jones, Christina Lindholm, Raj Mani, Steve Thomas and Ewan Wilkinson who were members of the original review advisory group; Gillian Leng, formerly of the Cochrane Peripheral Vascular Disease Group; Stephen Blair and Charles McCollum who provided useful feedback on the first version; Georg Waernhjelm who kindly translated a Swedish paper for us; and Andrew Herxheimer who provided useful comments on this review through the Comments and Criticism process.

For the previous review update we acknowledged the help and support of Martin Bland who advised regarding statistical interpretation of some of the included studies; and the respondents to our survey: Michael Clark, Michael Jünger, Mariani Fabrizio, Giovani Mosti, Steven E Zimmet, Elizabeth McGinnis, Hugo Partsch, Jeannie Donnelly and JJ Guex together with 12 anonymous respondents. We are very grateful to the following peer referees who provided valuable feedback on the draft of that update: Michael Clark, Michael Jünger and Gill Worthy. Thanks are also due to the following for assistance with translation: Gunilla Borglin, Mario Cruciani, Merja Jauhiainen, Paweł Kanturski, Angela Lai, Jesús López Alcalde and Joanna Zakrzewska.

For this review update we wish to thank the following for assistance with translation: Reynald Convert, Paweł Kanturski, Arturo MartíCarvajal, Elena Shek, Uwe Wollina and Li Xun.

We acknowledge the following for their contribution to the individual patient data meta-analysis: Peter Franks, Trevor Mole and Mark Scriven provided data and commented on the metaanalysis report for the purposes of its original publication (O'Meara 2009); Martin Bland contributed to data interpretation, checked the quality of the statistical analysis and made an intellectual contribution to the original meta-analysis report; Jayne Tierney gave advice on the meta-analysis protocol and data collection, checking and analysis and commented on the original metaanalysis report.

We would like to express appreciation for the support we have received from the staff of the Cochrane Wounds Group: to Ruth Foxlee for advising on the search strategy; to Sally Bell-Syer and Nicola Thomis for helpful advice when preparing the review; to Elizabeth Royle who copy-edited this review; and to Elizabeth McInnes and Gill Worthy who provided useful editorial feedback. 


\section{R E F E R E N C E S}

\section{References to studies included in this review}

Blecken 2005 \{published data only\}

Blecken SR, Villavicencio JL, Kao TC. Comparison of elastic versus nonelastic compression in bilateral venous ulcers: a randomized trial. Journal of Vascular Surgery 2005;42(6):1150-5.

\section{Brizzio 2010 \{published data only\}}

* Brizzio E, Amsler F, Lun B, Blättler W. Comparison of lowstrength compression stockings with bandages for the treatment of recalcitrant venous ulcers. Journal of Vascular Surgery 2010;51(2):410-6.

Brizzio EO. Comparison of low-strength compression stockings with bandages for the treatment of recalcitrant venous ulcers a randomized trial. W65, Abstract No. P42. The Symposium on Advanced Wound Care and the Wound Healing Society; 2010, April 17-20; Orlando, Florida. 2010.

Callam 1992b \{published data only\}

Callam MJ, Harper DR, Dale JJ, Brown D, Gibson B, Prescott RJ, et al. Lothian and Forth Valley leg ulcer healing trial, Part 1: elastic versus nonelastic bandaging in the treatment of chronic leg ulceration. Phlebology 1992;7(4):136-41.

Charles 1991 \{published data only\}

* Charles H. Compression healing of ulcers. Journal of District Nursing 1991;10(3):4-8.

Charles H. Short report: Compression healing of venous ulcers. Nursing Times 1992;88(3):52.

\section{Colgan 1995 \{published and unpublished data\}}

Colgan MP, Teevan M, McBride C, O'Sullivan L, Moore D, Shanik G. Cost comparisons in the management of venous ulceration. Proceedings of the 5th European Conference on Advances in Wound Management; 1995, 21-24 November; Harrogate, UK. London: Macmillan Magazines, 1995.

\section{Cordts 1992 \{published data only\}}

Cordts PR, Hanrahan LM, Rodriguez AA, Woodson J, LaMorte, WW, Menzoian JO. A prospective, randomized trial of Unna's boot versus Duoderm CGF hydroactive dressing plus compression in the management of venous leg ulcers. Journal of Vascular Surgery 1992;15(3):480-6.

\section{Danielsen 1998 \{published data only\}}

Danielsen L, Madsen SM, Henriksen L. Healing of venous leg ulcers: a randomized prospective study of a long-stretch versus short-stretch compression bandage [Heling af venøse bensår. En randomiseret prospektiv undersøgelse af en langstrœk - versus en kortstrœk-kompressionsbandage]. Ugeskrift for Laeger 1999;161(44):6042-5.

* Danielsen L, Madsen SM, Henriksen L. Venous leg ulcer healing: a randomized prospective study of long-stretch versus short-stretch compression bandages. Phlebology 1998;13:59-63.

Danielsen L, Madsen SM, Henriksen L, Sindrup J, Petersen LJ. Subbandage pressure measurements comparing a long-stretch with a short-stretch compression bandage. Acta DermatoVenereologica 1998;78(3):201-4.

DePalma 1999 \{published data only\}

DePalma RG, Kowallek D, Spence RK, Caprini JA, Nehler MR, Jensen $\mathrm{J}$, et al. Comparison of costs and healing rates of two forms of compression in treating venous ulcers. Vascular Surgery 1999;33(6):683-90.

\section{Duby 1993 \{published data only\}}

Duby T, Cherry G, Hoffman D, Cameron J, Doblhoff-Brown D, Ryan $\mathrm{T}$. A randomized trial in the treatment of venous leg ulcers comparing SSBs, 4LB system, and a long stretch-paste bandage system. 6th Annual Symposium on Advanced Wound Care. King of Prussia, PA: Health Management Publications, Inc, 1993.

* Duby T, Hoffman D, Cameron J, Doblhoff-Brown D, Cherry G, Ryan T. A randomized trial in the treatment of venous leg ulcers comparing SSBs, 4LB system, and a long stretch-paste bandage system. Wounds: A Compendium of Clinical Research and Practice 1993;5(6):276-9.

Duby T, Hoffman D, Cameron J, Doblhoff-Brown D, Ryan T, Cherry G. A randomised trial in the treatment of venous leg ulcers comparing SSBs, 4LB system, and a long stretch paste bandage system. 2nd European Conference on Advances in Wound Management; 1992, 20-23 October; Harrogate, UK. 1993.

\section{Eriksson 1984 \{published data only\}}

Eriksson G, Eklund A-E, Kallings LO. The clinical significance of bacterial growth in venous leg ulcers. Scandinavian Journal of Infectious Diseases 1984;16(2):175-80.

* Eriksson G, Eklund AE, Liden S, Zetterquist S. Comparison of different treatments of venous leg ulcers: a controlled study using stereophotogrammetry. Current Therapeutic Research 1984;35(4):678-84.

\section{Eriksson 1986 \{published data only\}}

Eriksson G. Comparative study of hydrocolloid dressings and double layer bandage in treatment of venous stasis ulceration. In: Ryan TJ editor(s). An environment for healing: The role of occlusion. London: Royal Society of Medicine International Congress and Symposium Series, 1984:111-3.

* Eriksson G. Comparison of two occlusive bandages in the treatment of venous leg ulcers. British Journal of Dermatology $1986 ; 114(2): 227-30$.

\section{Franks 2004 \{published and unpublished data\}}

Franks P, Moody M, Moffatt C. Randomised trial of four-layer and cohesive short-stretch compression in venous ulceration. Journal of Tissue Viability 2003;13(4):170.

* Franks PJ, Moody M, Moffatt CJ, Martin R, Blewett R, Seymour E, et al. Randomized trial of cohesive short-stretch versus four-layer bandaging in the management of venous ulceration. Wound Repair and Regeneration 2004;12(2):157-62.

Franks PJ, Moody M, Moffatt CJ, Patton J, Bradley L, Chaloner D, et al. Quality of life in a trial of short stretch versus four-layer 
bandaging in the management of chronic venous ulceration. Phlebology 2004;19(2):87-91.

Moffatt CJ, Moody M, Franks PJ. Randomised trial comparing four-layer with cohesive short stretch compression bandaging in the management of chronic venous ulceration. 13th Conference of the European Wound Management Association; 2003, 22-24 May; Pisa, Italy. 2003.

\section{Gould 1998 \{published data only\}}

Gould DJ, Campbell S, Harding E. A clinical evaluation of Setopress high compression bandage with Elastocrepe in the management of chronic venous ulceration (poster presentation). Proceedings of the 2nd European Conference on Advances in Wound Management. London: Macmillan Magazines, 1993.

Gould DJ, Campbell S, Harding EF. Short stretch vs. long stretch bandages in the management of chronic venous leg ulcers (conference abstract). Phlebology 1993;8(1):43.

* Gould DJ, Campbell S, Newton H, Duffelen P, Griffin M, Harding EF. Setopress vs Elastocrepe in chronic venous ulceration. British Journal of Nursing 1998;7(2):66-73.

\section{Harley 2004 \{published data only\}}

Harley J, Harcourt D, Hutchinson B, Liew I. A comparative trial of two layer compression bandaging versus four layer compression bandaging in the treatment of chronic venous ulcers. First World Wound Healing Congress; 10-13 September 2000; Melbourne, Australia. 2000.

* Harley J, Harcourt D, Hutchinson B, McLean M, Long M. A comparative trial of long stretch compression bandaging versus multi-layer compression bandaging in the treatment of chronic venous ulcers. Primary Intention 2004;12(1):6-13.

\section{Hendricks 1985 \{published data only\}}

Hendricks WM, Swallow RT. Management of stasis leg ulcers with Unna's boots versus elastic support stockings. Journal of the American Academy of Dermatology 1985;12(1):90-8.

\section{Iglesias 2004 \{published and unpublished data\}}

* Iglesias C, Nelson EA, Cullum NA, Torgerson DJ, on behalf of the VenUS Team. VenUS I: a randomised controlled trial of two types of bandage for treating venous leg ulcers. Health Technology Assessment 2004;8(29):1-120.

Iglesias CP, Nelson EA, Cullum N, Torgerson DJ, on behalf of the VenUS I collaborators. Economic analysis of VenUS I, a randomized trial of two bandages for treating venous leg ulcers. British Journal of Surgery 2004;91(10):1300-6.

Iglesias CP, Nelson EA, Torgerson DJ, Cullulm N. Economic analysis of VenUS I: a randomised controlled trial of two bandages for treating venous leg ulcers. 2nd World Union of Wound Healing Societies, Paris, France. 2004.

Nelson EA, Iglesias CP, Cullum N, Torgerson DJ, on behalf of the VenUS I collaborators. Randomized clinical trial of four-layer and short-stretch compression bandages for venous leg ulcers (VenUS I). British Journal of Surgery 2004;91(10):1292-9.

\section{Jünger 2004a \{published data only\}}

Gardon-Mollard C, Jünger M, Partsch H, Zuccarelli F, Taupin V. Efficacy of a short-stretch tubular compression orthosis compared to a non-elastic bandage in the treatment of venous ulcers. 13th Conference of the European Wound Management Association; 22-24 May 2003; Pisa, Italy. 2003.

Gardon-Mollard C, Jünger M, Partsch H, Zuccarelli F, Taupin V. Efficacy of a tubular compression orthosis compared to a SSB in the treatment of venous ulcers. Phlebology 2003;18(3):152.

Jünger M, Partsch H, Ramelet A-A, Zuccarelli F. Efficacy of a new compression device versus bandages in the treatment of venous leg ulcers. 2nd World Union of Wound Healing Societies' Meeting; July 2004; Paris, France. 2004.

* Jünger M, Partsch H, Ramelet A-A, Zuccarelli F. Efficacy of a ready-made tubular compression device versus short-stretch compression bandages in the treatment of venous leg ulcers. Wounds: A Compendium of Clinical Research and Practice 2004;16(10):313-20.

\section{Jünger 2004b \{published and unpublished data\}}

Jünger M, Kohnen R, Stahl H-W. Efficacy and cost effectiveness of a compression stocking system for treatment of venous leg ulcers. 17th Conference of the European Wound Management Association; May 2007; Glasgow, UK. 2007.

Jünger M, Wollina U. Compression therapy of venous leg ulcers with the compression stocking Venotrain ${ }^{\circledR}$ ulcertec: initial data from a prospective randomised study [Kompressionstherapie bei venösem ulcus cruris mit dem ulkus-kompressionsstrumpf Venotrain ${ }^{\circledast}$ ulcertec: erste daten aus einer prospektiven randomisierten studie]. Vasomed 2002;14(4):158-9.

* Jünger M, Wollina U, Kohnen R, Rabe E. Efficacy and tolerability of an ulcer compression stocking for therapy of chronic venous ulcer compared with a below-knee compression bandage: results from a prospective, randomized, multicentre trial. Current Medical Research and Opinions 2004;20(10):1613-23.

Jünger M, Wollina U, Kohnen R, Rabe E. Efficacy of a compression stocking (Venotrain ${ }^{\circledR}$ Ulcertec) for therapy of venous leg ulcers. Proceedings of the 15th World Congress International Union of Phlebology; October 2005; Rio de Janiero, Brazil. 2005.

\section{Kikta 1988 \{published data only\}}

Kikta MJ, Schuler JJ, Meyer JP, Durham JR, Eldrup-Jorgensen J, Schwarcz TH, et al. A prospective, randomized trial of Unna's boots versus hydroactive dressing in the treatment of venous stasis ulcers. Journal of Vascular Surgery 1988;7(3):478-83.

\section{Knight 1996 \{published and unpublished data\}}

${ }^{\star}$ Knight CA, McCulloch J. A comparative study between two compression systems in the treatment of venous insufficiency in leg ulcers. 9th Annual Symposium on Advanced Wound Care and 6th Annual Medical Research Forum on Wound Repair; 1996, April 20-24; Atlanta. Pennsylvania: Wayne, 1996:117. 
Knight CA, McCulloch JM. A comparative study between two compression systems in the treatment of venous insufficiency leg ulcers. Unpublished report 1996.

\section{Koksal 2003 \{published data only\}}

Koksal C, Bozkurt AK. Combination of hydrocolloid dressing and medical compression stocking versus Unna's boot for the treatment of venous leg ulcers. Swiss Medical Weekly 2003;133(22-26):364-8.

\section{Kralj 1996 \{published and unpublished data\}}

Kralj B, Kosicek M. Randomised comparative trial of single-layer and multi-layer bandages in the treatment of venous leg ulcer. Proceedings of the 6th European Conference on Advances in Wound Management; 1995, 21-24 November; Harrogate, UK. London: Macmillan Magazines, 1996:158-60.

\section{Mariani 2008 \{published data only\}}

Mancini S, Bucalossi M, Mariani F. Elastic stocking versus bandage. A multicentric randomised trial. EWMA Conference, 20-22 May 2009, Helsinki, Finland. EWMA Journal 2009;9(2):Abstract P193.

* Mariani E, Mattaliano V, Mosti G, Gasbarro V, Bucalossi M, Blättler W, et al. The treatment of venous leg ulcers with a specifically designed compression stocking kit: comparison with bandaging. Phlebologie 2008;37:191-7.

\section{Meyer 2002 \{published data only\}}

* Meyer FJ, Burnand KG, Lagattolla NRF, Eastham D. Randomized clinical trial comparing the efficacy of two bandaging regimens in the treatment of venous leg ulcers. British Journal of Surgery 2002;89(Pt 1):40-4.

Ruckley CV, Callam MJ, Harper DR, Dale JJ, Gibson B, Prescott RJ. Randomized clinical trial comparing the efficacy of two bandaging regimens in the treatment of venous leg ulcers (Br J Surg 2002; 89: 40-4). Letter 1. British Journal of Surgery 2002;89(6):810.

\section{Meyer 2003 \{published data only\}}

Meyer FJ, Burnand KG, Lagatolla NRF, Eastham D. More venous leg ulcers are healed by three-layer paste than by 4LBs: a randomised, controlled, prospective study. Proceedings of the First World Wound Healing Congress; 10-13 September, 2000. Melbourne, Australia, 2000:74-5.

* Meyer FJ, McGuinness CL, Lagattolla NRF, Eastham D, Burnand KG. Randomized clinical trial of three-layer paste and 4LBs for venous leg ulcers. British Journal of Surgery 2003;90(8):934-40.

Nelson EA. 3-layer paste bandages were more effective than 4 layer bandages for healing venous leg ulcers. Evidence Based Nursing 2004;7:21.

\section{Milic 2007 \{published data only\}}

Milic DJ, Zivic SS, Bogdanovic DC, Perisic ZD, Milosevic ZD, Jankovic RJ, et al. A randomized trial of the Tubulcus multilayer bandaging system in the treatment of extensive venous ulcers. Journal of Vascular Surgery 2007;46(4):750-5.
Milic 2010 \{published data only\}

Milic DJ, Zivic SS, Bogdanovic DC, Jovanovic MM, Jankovic RJ, Milosevic ZD, et al. The influence of different sub-bandage pressure values on venous leg ulcers healing when treated with compression therapy. Journal of Vascular Surgery 2010;51(3):655-61.

\section{Moffatt 1999 \{published data only\}}

Franks PJ, Moffatt CJ, Ellison DA, Connolly M, Fielden S, Groarke L, et al. Quality of life in venous ulceration: a randomized trial of two bandage systems. Phlebology 1999;14:95-9.

McCollum CN, Ellison DA, Groarke L, Fielden S, Connolly M, Franks PJ, et al. Randomised trial comparing Profore and the original 4LB in the treatment of venous leg ulceration. European Wound Management Association Conference; 1997, 27-29 April; Milan, Italy. London: Macmillan Magazines, 1997:30.

* Moffatt CJ, Simon DA, Franks PJ, Connolly M, Fielden S, Groarke L, at al. Randomised trial comparing two 4LB systems in the management of chronic leg ulceration. Phlebology 1999;14:139-42.

\section{Moffatt 2003a \{published and unpublished data\}}

Franks PJ, Stevens J, Hourican C, Doherty D, O'Connor T, McCullagh L, et al. Quality of life in venous ulceration: use of the SF-36 in a randomised trial of two bandage systems. 11th Conference of the European Wound Management Association; 17-19 May 2001; Dublin, Ireland. 2001.

Franks PJ, Stevens J, Hourican C, Doherty DC, O'Connor T, McCullagh L, et al. Quality of life in venous ulceration: use of the SF-36 in a randomized trial of two bandage systems (abstract only). Ostomy/Wound Management 2002;48(4):73.

Moffatt CJ, McCullagh L, O'Connor T, Doherty DC, Hourican C, Lewis $C$, et al. Randomised trial comparing four layer with two layer high compression bandaging in the management of chronic leg ulceration. 11th Conference of the European Wound Management Association; 2001, 17-19 May; Dublin, Ireland. 2002.

* Moffatt CJ, McCullagh L, O'Connor T, Doherty DC, Hourican C, Stevens J, et al. Randomized trial of four-layer and two-layer bandage systems in the management of chronic venous ulceration. Wound Repair and Regeneration 2003;11(3):166-71.

\section{Moffatt 2008 \{published data only\}}

Moffatt C, Edwards L, Harding K, Collier M, Treadwell T, Miller M, et al. A randomized, crossover clinical trial of a two-layer and four-layer compression bandage system in the treatment of venous leg ulcers. 3rd Congress of the World Union of Wound Healing Societies Meeting; 2008, 4-8 June; Toronto, Canada. 2008.

* Moffatt CJ, Edwards L, Collier M, Treadwell T, Miller M, Shafer L, et al. A randomised controlled 8-week crossover clinical evaluation of the $3 \mathrm{M}^{\mathrm{TM}}$ Coban ${ }^{\mathrm{TM}} 2$ layer compression system versus Profore ${ }^{\mathrm{TM}}$ to evaluate the product performance in patients with venous leg ulcers. International Wound Journal 2008;5(2):267-79. 
Price P, Harding K, Moffatt C. Randomized, cross-over, clinical trial to compare the impact of two-layer versus a four-layer compression bandage system in the treatment of venous leg ulcer. 18th Conference of the European Wound Management Association; 14-16 May 2008; Lisbon, Portugal. 2008.

Tucker JA. A prospective, multi-site, randomized, cross-over, clinical trial of a two-layer and a four-layer compression bandage system in the treatment of venous leg ulcers. Scientific and clinical abstracts from the 40th Annual Wound, Ostomy and Continence Nurses Annual Conference. Journal of Wound, Ostomy and Continence Nursing. 2008; Vol. 35:S71.

\section{Moody 1999 \{published data only\}}

Moody M. Comparison of Rosidal K and Surepress in the treatment of venous leg ulcers. British Journal of Nursing 1999;8(6):345-55.

\section{Morrell 1998 \{published and unpublished data\}}

Morrell CJ. Randomised controlled trial of four-layer compression bandaging. Unpublished report 1996.

Morrell CJ, King B, Brereton L. Community-based leg ulcer clinics: organisation and cost-effectiveness. Nursing Times 1998;94(9):51-4.

* Morrell CJ, Walters SJ, Dixon S, Collins KA, Brereton LML, Peters J, et al. Cost effectiveness of community leg ulcer clinics: randomised controlled trial. BMJ 1998;316(7143):1487-91.

\section{Nelson 2007a \{published data only\}}

Dale JJ, Ruckley CV, Harper DR, Gibson B, Nelson EA, Prescott RJ. A factorial trial of drugs, dressings and bandages in the treatment of leg ulcers. Proceedings of the 5th European Conference on Advances in Wound Management; 1995, 21-24 November; Harrogate, UK. 1996:193-4.

Franks PJ, Bosanquet N, Brown D, Straub J, Harper DR, Ruckley CV. Perceived health in a randomised trial of treatment for chronic venous ulceration. European Journal of Vascular and Endovascular Surgery 1999;17(2):155-9.

Nelson EA, Harper DR, Ruckley CV, Prescott RJ, Gibson B, Dale JJ. A randomised trial of single layer and multi-layer bandages in the treatment of chronic venous ulceration. Phlebology 1995;Suppl 1:915-6.

* Nelson EA, Prescott RJ, Harper DR, Gibson B, Brown D, Ruckley CV. A factorial, randomized trial of pentoxifylline or placebo, four-layer or single-layer compression, and knitted viscose or hydrocolloid dressings for venous ulcers. Journal of Vascular Surgery 2007;45(1):134-41.

\section{O'Brien 2003 \{published data only\}}

Clarke-Moloney M, O'Brien JF, Grace PA, Burke PE. Healthrelated quality of life during four-layer compression bandaging for venous leg ulcer disease: a randomised controlled trial. Irish Journal of Medical Science 2005;174(2):21-5.

O'Brien JF, Grace PA, Perry IJ, Burke PE. Randomised controlled trial: cost-effectiveness of four-layer compression bandaging in venous leg ulcer care. 11th Conference of the European Wound
Management Association; 2001 17-19 May; Dublin, Ireland. 2001.

* O'Brien JF, Grace PA, Perry IJ, Hannigan A, Clarke-Moloney M, Burke PE. Randomized clinical trial and economic analysis of four-layer compression bandaging for venous ulcers. British Journal of Surgery 2003;90(7):794-8.

Partsch 2001 \{published and unpublished data\}

Partsch H, Damstra RJ, Tazelaar DJ, Schuller-Petrovic S, Velders AJ, de Rooij MJM, et al. Multicentre, randomised controlled trial of four-layer bandaging versus short-stretch bandaging in the treatment of venous leg ulcers. VASA 2001;30(2):108-113.

\section{Polignano 2004a \{published and unpublished data\}}

Allegra C, Cariotti R, Bonadeo P, Gasbarro S, Cataldi R, Polignano R, et al. Four-layer compared with Unna's Boot in venous leg ulcer management. 11th Conference of the European Wound Management Association. Dublin, Ireland. 2001.

Allegra C, Cariotti R, Bonadeo P, Gasbarro S, Cataldi R, Polignano R, et al. Four-layer compared with Unna's Boot in venous leg ulcer management. 11th Annual Meeting of the European Tissue Repair Society. Cardiff, UK. Wound Repair and Regeneration. 2001; Vol. 9, issue 5:393.

* Polignano R, Bonadeo P, Gasbarro S, Allegra C. A randomised controlled study of four-layer compression versus Unna's Boot for venous ulcers. Journal of Wound Care 2004;13(1):21-4.

\section{Polignano 2004b \{published data only\}}

Polignano R, Guarnera G, Bonadeo P. Evaluation of SurePress Comfort: a new compression system for the management of venous leg ulcers. Journal of Wound Care 2004;13(9):387-91.

\section{Rubin 1990 \{published data only\}}

Rubin JR, Alexander J, Plecha EJ, Marman C. Unna's boot vs polyurethane foam dressings for the treatment of venous ulceration. A randomized prospective study. Archives of Surgery 1990;125(4):489-90.

\section{Scriven 1998 \{published and unpublished data\}}

Scriven JM, Taylor LE, Wood AJ, Bell PRF, Naylor AR, London NJM. A prospective randomised trial of four-layer versus short stretch compression bandages for the treatment of venous leg ulcers. Annals of the Royal College of Surgeons 1998;80(3):215-20.

\section{Szewczyk 2010 \{published data only\}}

Szewczyk MT, Jawień A, Cierzniakowska K, Cwajda-Białasik J, Mościcka P. Comparison of the effectiveness of compression stockings and layer compression systems in venous ulceration treatment. Archives of Medical Science 2010;6(5):793-9.

Taradaj 2007 \{published data only\}

Taradaj J, Franek A, Dolibog P, Cierpka L, Blaszczak E. The impact of the sonography and compression therapy on enhancement of healing venous leg ulcers after surgical treatment [Wplyw sono-i kompresoterapil na wspomaganie 
gojenia owrzodzen zylnych goleni po leczeniu chirurgicznym]. Polski Merkuriusz Lekarski 2007;23(138):426-9.

\section{Taradaj 2009 \{published data only\}}

Taradaj J, Franek A, Brzezinska-Wcislo L, Blaszczak E, Polak A. Randomized trial of medical compression stockings versus twolayer short-stretch bandaging in the management of venous leg ulcers. Phlebologie 2009;38(4):157-63.

\section{Taylor 1998 \{published data only\}}

Taylor A. A prospective study to compare healing rates and associated treatment costs for current management of venous leg ulcers in the community and a four-layer compression bandage regime. Proceedings of the 4th European Conference on Advances in Wound Management. London: Macmillan Magazines, 1995:199-200.

Taylor A, Taylor R, Marcuson R. Comparative healing rates and cost of conventional and four-layer treatment of venous ulcers.. Phlebology 1995;10:85.

* Taylor AD, Taylor RJ, Marcuson RW. Prospective comparison of healing rates and therapy costs for conventional and four layer high compression bandaging treatments of venous leg ulcers. Phlebology 1998;13:20-4.

Taylor AD, Taylor RJ, Marcusson RW. Prospective comparison of healing rates and therapy costs for conventional and four layer compression bandaging treatments of venous leg ulcers. Surgical Directorate, Central Manchester Healthcare NHS Trust.

\section{Travers 1992 \{published data only\}}

Travers JP, Dalziel KL, Makin GS. Assessment of a new one-layer adhesive bandaging method in maintaining prolonged limb compression and effects on venous ulcer healing. Phlebology 1992; 7:59-63.

\section{Ukat 2003 \{published and unpublished data\}}

Ukat A, Konig M, Vanscheidt W, Münter K-C. Short-stretch versus multilayer compression for venous leg ulcers: a comparison of healing rates. Journal of Wound Care 2003;12(4):139-43.

\section{Vowden 2000 \{published and unpublished data\}}

Vowden $\mathrm{K}$. A randomized study comparing compliance, healing and complications from three alternative $4 \mathrm{LB}$ regimens in the treatment of venous leg ulcers. 9th European Conference on Advances in Wound Management; 9-11 November, 1999; Harrogate, UK. 1999.

* Vowden KR, Mason A, Wilkinson D, Vowden P. Comparison of the healing rates and complications of three $4 \mathrm{LB}$ regimens. Journal of Wound Care 2000;9(6):269-72.

\section{Wilkinson 1997 \{published and unpublished data\}}

Wilkinson E, Buttfield S, Cooper S, Young E. Randomised controlled trial of two systems of compression bandaging for chronic venous leg ulcers. Unpublished report 1996.

* Wilkinson E, Buttfield S, Cooper S, Young E. Trial of two bandaging systems for chronic venous leg ulcers. Journal of Wound Care 1997;6(7):339-40.

\section{Zuccarelli 1997 \{published and unpublished data\}}

Zuccarelli F, Allaert FA. Effectivity of support in the treatment of leg ulcers. A comparative multicentre study of elastic bandage (BIFLEX ${ }^{\circledR}$ ) versus extensive bandage [Efficacité de la contention dans le traitement des ulcères de jambe. Etude multicentrique comparant une bande élastique (BIFLEX ${ }^{\circledR}$ ) et une bande extensible]. Angéiologie 1997;49(5):15-8.

\section{References to studies excluded from this review}

Alvarez 2005 \{published data only\}

Alvarez O, Markowitz L, Booker J, Rogers R, Waltrous L, Patel M. A randomized clinical trial to evaluate healing of chronic venous ulcers in ambulatory patients treated with modified unna's boot and four layer compression bandage. European Wound Management Association Conference; 2005, 15-17 September; Stuttgart, Germany. 2005.

Baccaglini 1998 \{published data only\}

Baccaglini U, Giraldi E, Spreafico G, Sorrentino P, Castoro C, Penzo S, et al. Evaluation of the Jobst UlcerCare system for the treatment of venous ulcers. Phlebology 1998;13(4):163-5.

Blair 1988 \{published data only\}

Blair SD, Wright DDI, Backhouse CM, Riddle E, McCollum CN. Sustained compression and healing of chronic venous ulcers. BMJ 1988;297:1159-61.

\section{Brizzio 2006 \{published data only\}}

Brizzio EO, Blttler W, Rossi G, Chirinos A, Cantero I, Idiazabal G, et al. Healing venous leg ulcers with different modalities of leg compression. Phlebologie 2006;35:349-55.

Cameron 1996 \{published data only\}

Cameron J, Hofman D, Poore S, Duby T, Cherry G, Ryan T. A comparative study of two bandage systems. Proceedings of the 3rd European Conference on Advances in Wound Management. London: Macmillan Magazines, 1994:168.

* Cameron J, Hofman D, Poore S, Duby T, Cherry G, Ryan T. A retrospective trial in the treatment of venous leg ulcers. Wounds 1996;8(3):95-100.

\section{Cherry 1990 \{published data only\}}

Cherry G. Clinical comparison of a new compression bandage. Nursing Standard 1990;8 (Suppl):8-11.

\section{Falanga 1998 \{published data only\}}

* Falanga V, Margolis D, Alvarez O, Auletta M, Maggiacomo F, Altman M, et al. Rapid healing of venous ulcers and lack of clinical rejection with an allogenic cultured human skin equivalent. Archives of Dermatology 1998;134:293-300.

Falanga V, Saap LJ, Ozonoff A. Wound bed score and its correlation with healing of chronic wounds. Dermatologic Therapy 2006;19:383-90.

\section{Fuessl 2009 \{published data only\}}

Fuessl HS. Which bandage for venous leg ulcer? [Welcher verband beim ulcus cruris?]. MMW-Fortschritte Der Medizin 2009;151(34-3):28 
Hamel-Desnos 2010 \{published data only\}

Hamel-Desnos CM, Guias BJ, Desnos PR, Mesgard A. Foam sclerotherapy of the saphenous veins: randomised controlled trial with or without compression. European Journal of Vascular and Endovascular Surgery 2010;39:500-7.

Heinen 2010 \{published data only\}

Heinen M, Borm G, van Achterberg T. 'Lively Legs': increased physical activity and fewer wound days in venous leg ulcer patients (abstract). Fourth European Nursing Congress. Journal of Clinical Nursing 2010;19(Supplement):42.

\section{Hjerppe 2010 \{published data only\}}

Hjerppe A, Saarinen JP, Venermo MA, Huhtala HS, Vaalasti A. Prolonged healing of venous leg ulcers: the role of venous reflux, ulcer characteristics and mobility. Journal of Wound Care 2010;19(11):474-84.

\section{Horakova 1994 \{published data only\}}

Horakova M, Partsch H. Venous leg ulcers: are compression bandages indicated?. Phlébologie 1994;47:53-7.

Partsch $\mathrm{H}$, Horakova MA. Compression stockings for the treatment of venous leg ulcers [Kompressionstrumpfe zur Behandlung venoser Unterschenkelgeschwure]. Wiener Medizine Wochenschrift 1994;144:242-9.

\section{Ivanovic 2011 \{published data only\}}

Ivanovic N, Panfil E-M. Compression in varicose ulcer. What is the effect of this measure? [Kompression bei Ulcus cruris venosum. Welchen effekt hat die Maßnahme?]. Pflegewissenschaft 2011;64(7):412.

\section{Jull 2009 \{published data only\}}

Jull A, Walker N, Parag V, Molan P, Rodgers A, on behalf of the Honey as Adjuvant Leg Ulcer Therapy (HALT) trial collaborators. Venous ulcer management in New Zealand: usual care versus guideline recommendations. Journal of the New Zealand Medical Association 2009;122(1295):9-18.

\section{Jünger 2006 \{published data only\}}

Jünger M, Riebe H. A world's novelty: Venotrain micro balance combining compression therapy with effective skin care a randomised, controlled, prospective, explorative study. European Venous Forum. London, UK. 29 June-1 July 2006.

\section{Kucharzewski 2003 \{published data only\}}

Kucharzewski M, Ślezak A, Franek A. Topical treatment of nonhealing venous leg ulcers by cellulose membrane. Phlebologie 2003;32:147-51.

\section{Kuznetsov 2009 \{published data only\}}

Kuznetsov NA, Rodoman GV, Nikitin VG, Karev MA, Shalayeva TI. The use of current bandages in the treatment of patients with venous trophic ulcers of the shin: clinical and economic aspects. Khirugiia Moskva 2009;11:63-9.

\section{Lee 2009 \{published data only\}}

Lee G, Rajendran S, Anand S. New single-layer compression bandage system for chronic venous leg ulcers. British Journal of Nursing (Tissue Viability Supplement) 2009;18(15):S4-S18.
Luo 2009 \{published data only\}

Luo Y-W, Yang Y, Xin S-J. Graduated compression stockings in the therapy of chronic venous insufficiency (482 reports). Journal of Dalian Medical University 2009;31(6):692-4.

\section{Marston 1999 \{published data only\}}

Marston WA, Carlin RE, Passman MA, Farber MA, Keagy BA. Healing rates and cost efficacy of outpatient compression treatment for leg ulcers associated with venous insufficiency. Journal of Vascular Surgery 1999;30:491-8.

\section{Nissinen-Paatsamala 1995 \{published data only\}}

Nissinen-Paatsamala K, Jansén C. Low elastic long-time bandaging - intensive care of varicose ulcer with diminished personnel. Suomen Lääkärilehti 1995;50:717-9.

Northeast 1990 \{published and unpublished data\}

Northeast ADR, Layer GT, Wilson NM, Browse NL, Burnand KG. Increased compression expedites venous ulcer healing. Royal Society of Medicine Venous Forum, 1990 October Meeting. 1990.

\section{Olofsson 1996 \{published data only\}}

Olofsson B, Ljunghall K, Nordin-Bjorklund K, Sorensen S, Leppert J. Two therapeutic models in venous leg ulcers are compared: better results with optimized compression [Optimerad kompression ger battre resultat]. Lakartidningen 1996;93(51-52):4752-4.

\section{Partsch 2008a \{published data only\}}

Partsch H, Vanscheidt W, Ukat A. Dose-response relationship in randomized clinical studies of compression therapy for venous insufficiency oedema. Phlebology 2008;23:233-4.

\section{Robson 2004 \{published data only\}}

Brennan M, Serena TE, Hanft J, Snyder R. Patients with venous leg ulcerations have diminished quality of life: analysis of a multi-centre randomized clinical trial. The Symposium on Advanced Wound Care and the Wound Healing Society. 2010, April 17-20.

* Robson MC, Hanft J, Garner W, Jenson J, Serena T, Payne WG, et al. Healing of chronic venous ulcers is not enhanced by the addition of topical repifermin (KGF-2) to standardized care. The Journal of Applied Research 2004;4(2):302-11.

\section{Russo 1999 \{published data only\}}

Russo A, Roberta C. Multi-layer versus two-layer bandage system in the treatment of venous leg ulcers. A comparative study. 9th European Conference on Advances in Wound Management; 1999, 9-11 November; Harrogate UK. 1999.

Sabolinski 1995 \{published data only\}

* Sabolinski M, Borchard G, Mulder G. Cost benefit analysis of living skin equivalent versus graduated, multi-layered compression therapy for the treatment of venous skin ulcers. Diabetes 1995;44(Suppl 1):107.

Sabolinski M, Borchard G, Mulder G. Cost-benefit analysis of Living Skin Equivalent versus graduated, multi-layered compression therapy for the treatment of venous skin ulcers. Proceedings of the 5th European Conference on Advances in 
Wound Management; 1995, 21-24 November; Harrogate, UK. London: Macmillan Magazines, 1996:107-9.

\section{Scriven 2000 \{published data only\}}

Scriven JM, Bello M, Taylor LE, Wood AJ, London NJM. Studies of a new multi-layer compression bandage for the treatment of venous ulceration. Journal of Wound Care 2000;9(3):143-7.

\section{Serra 2010 \{published data only\}}

Serra N, Palomar F, Fornes B, Capillas R, Berenguer M, Aranda J, et al. Effectiveness of the association of multilayer compression therapy and periwound protection with Cavilon $^{\circledR}$ (no sting barrier film) in the treatment of venous leg ulcers [Efectividad del tratamiento de las úlceras venosas con vendaje compresivo multicapa asociado a protección de la piel perilesional con Cavilon ${ }^{\circledR}$ (película barrera no irritante)]. Gerokomos 2010;21(3):124-30.

\section{Sikes 1985 \{published data only\}}

Sikes E. Evaluation of a transparent dressing in the treatment of stasis ulcers of the lower limb. Journal of Enterostomal Therapy 1985;12:116-20.

\section{Sironi 1994 \{published data only\}}

* Sironi G, Losa S, Di Luca G, Pezzoni F. Patients with venous leg ulcers in vascular surgery treatment with Intrasite gel, Opsite Flexigrid, Allevyn and Flexobande Legere/Forte: a randomised comparative clinical evaluation - an interim report (poster presentation). 13th Conference of the European Wound Management Association; 2003, 22-24 May; Pisa, Italy. 2003.

Sironi G, Losa S, DiLuca G, Pezzoni F. Treatment of venous leg ulcers with Intrasite gel, Opsite Flexigrid, Allevyn hydrocellular dressing and Flexobande (elastic compression bandage) in vascular surgery. A protocol for clinical evaluation. 3rd European Conference on Advances in Wound Management; 1993, 19-22 October; Harrogate, UK. London: Macmillan Magazines, 1994:164.

\section{Smith Strom 2006 \{published data only\}}

Smith Strom H. Comparison of healing and costs with two different treatments. A randomized controlled study of patients with leg ulcers, treated in primary health care [Norwegian]. Vard I Norden, Nursing Science and Research in the Nordic Countries 2006;26(2):4-9.

\section{Szewczyk 2009 \{published data only\}}

Jawien A, Szewczky MT, Banaszkiewicz Z, Moscicka P, Hancke E. Pain associated with venous ulcers and its relation to dynamics of wound healing. EWMA Journal 2010;10(2):42.

Jawien A, Szewczyk MT, Mościcka P, Cierzniakowska K, Cwajda J. Evaluation of clinical effectiveness of two- and fourlayer compression in venous leg ulcer treatment: randomized study. European Venous Forum abstracts. Phlebology 2008;23:234-5

* Szewczyk MT, Jawień A, Migdalski A, Piotrowicz R, Grzela T, Brazis P. Predicting time to healing by anatomical assessment of venous pathology. Medical Science Monitor 2009;15(2):CR74CR81.
Torra i Bou 2003 \{published data only\}

Torra i Bou JE, Rueda Lopez J, Blanco Blanco J, Torres Ballester J, Toda Lloret L. Venous ulcers. Multilayer compression system or crepe bandage? Comparative study on effectiveness, cost and impact on quality of life [Spanish]. Revista de Enfermeria 2003;26(9):59-66.

\section{Van Laere 2010 \{published data only\}}

Van Laere M. Comparative study between 2 short stretch compression systems. EWMA Conference, 26-28 May 2010, Geneva, Switzerland. EWMA Journal 2010;10(2):149, Abstract 173.

\section{Vowden 2001 \{published data only\}}

Vowden KR, Wilkinson D, Vowden P. The K-Four bandage system: evaluating its effectiveness on recalcitrant venous leg ulcers. Journal of Wound Care 2001;10(5):182-4.

\section{Walker 1996 \{published data only\}}

Walker P, Faria DT. Evaluation of a three layer bandage system versus a 4LB system in venous leg ulcers. Symposium on Advanced Wound Care and Medical Research Forum on Wound Repair. Wayne: Health Management Publications, 1996:121.

\section{Zamboni 2004 \{published data only\}}

* Zamboni P, Cisno C, Marchetti F, Mazza P, Fogato L, Carandina S, et al. Haemodynamic CHIVA correction surgery versus compression for primary venous ulcers: first year results. Phlebology 2004;19(1):28-34.

Zamboni P, Cisno C, Marchetti F, Mazza P, Fogato L, Carandina S, et al. Minimally invasive surgical management of primary venous ulcers vs. compression treatment: a randomized clinical trial. European Journal of Vascular and Endovascular Surgery 2003;25(4):313-8.

\section{References to studies awaiting assessment}

Bertaux 2010 \{published data only\}

Bertaux E, Ettner N. A new approach to treat venous leg ulcers by compression therapy. EWMA Conference, 26-28 May 2010, Geneva, Switzerland. EWMA Journal. 2010; Vol. 10, issue 2:40, Abstract 39

\section{Harrison 2011 \{published data only\}}

Harrison MB. The Canadian bandaging trial: A multi-site RCT of bandaging technologies for venous leg ulcers. The 2011 International Nursing Research Conference; 16-18 May, 2011; Harrogate, UK. 2011.

* Harrison MB, VanDenKerkhof EG, Hopman WM, Graham ID, Carley ME, EA Nelson for the Canadian Bandaging Group. The Canadian Bandaging Trial: evidence-informed leg ulcer care and the effectiveness of two compression technologies. BMC Nursing 2011;10(20):available from http:// www.biomedcentral.com/1472-6955/10/20 (accessed June 2012).

Smith LJ, Harrison MB, Graham ID, Lamb M. Community leg ulcer bandaging study: lessons learned in a pilot, randomized controlled trial. Ostomy Wound Management 2010;56(9):32-42. 


\section{Moffatt 2003b \{published data only\}}

Moffatt CJ. A multi-centre randomised study comparing a new innovative vari-stretch compression bandaging system with a traditional multi-layer compression bandage system for the management of venous leg ulcers [Abstract]. 13th Conference of the European Wound Management Association; 2003, 22-24 May; Pisa, Italy. 2003:111.

\section{Mosti 2010 \{published data only\}}

Mosti G, Mattaliano V, Conte R, Abel M. Promotion of healing, pain relief, tolerability and quality of life: results of a prospective, controlled, randomized comparison study with two wound dressings in out-patients with non-infected leg ulcers. EWMA Conference, 26-28 May 2010, Geneva, Switzerland. EWMA Journal. 2010; Vol. 10, issue 2:59, Abstract 77.

\section{Mosti 2011 \{published data only\}}

Mosti G, Crespi A, Mattaliano V. Comparison between a new, two-component compression system with zinc paste bandages for leg ulcer healing: a prospective, multicenter, randomized, controlled trial monitoring sub-bandage pressures. Wounds: $A$ Compendium of Clinical Research \& Practice 2011;23(5):126-34.

\section{Taradaj 2011 \{published data only\}}

Taradaj J, Franek A, Cierpka L, Brzezinska-Wcislo L, Blaszczak E, Polak A, et al. Early and long-term results of physical methods in the treatment of venous leg ulcers: randomized controlled trial. Phlebology 2011;26(6):237-45.

\section{Wong 2012 \{published data only\}}

Andriessen A, Wong IKY, Lee DTF, Wong LY, Chao DVK, Heung NF, et al. RCT comparing treatment outcome and quality of life of two compression bandaging systems and standard care without compression in venous leg ulcer patients (abstract). EWMA Journal 2011;11(2 Suppl):61.

Wong IKY, Andriessen A, Charles HE, Thompson D, Lee DTF, So WKW, et al. Randomized controlled trial comparing treatment outcome of two compression bandaging systems and standard care without compression in patients with venous leg ulcers. Journal of the European Academy of Dermatology and Venereology 2012;26:102-10.

* Wong IKY, Andriessen A, Lee DTF, Thompson D, Wong LY, Chao DVK, et al. Randomized controlled trial comparing treatment outcome of two compression bandaging systems and standard care without compression in patients with venous leg ulcers. Journal of Vascular Surgery 2012;55(5):1376-85.

\section{References to ongoing studies}

\section{Dumville 2009 \{published data only\}}

Dumville J. VenUS IV: Compression hosiery versus compression bandaging in the treatment of venous leg ulcers. Details available from http://www.controlled-trials.com/ ISRCTN49373072/dumville and http://www.hta.ac.uk/1766 (both accessed 10th November 2011).

\section{Matos de Abreu 2011 \{published data only\}}

Matos de Abreu A, de Oliveira BGRB. Study of Unna's boot compared to use of elastic bandages in patients with venous ulcers - clinical trial [Bota de Unna comparada à bandagem elástica em portadores de úlceras venosas: ensaio clínico]. Online Brazilian Journal of Nursing 2011;10(3):Available from http://www.objnursing.uff.br/index.php/nursing/article/ view/3311 (accessed 2nd July 2012).

Weller 2010 \{published data only\}

Weller C, Evans S, Jolley D, McNeil J. A randomised controlled trial to evaluate elastic versus inelastic compression for the treatment of venous ulcers (abstract). EWMA Journal 2011;11(2 Suppl):84

* Weller CD, Evans S, Reid CM, Wolfe R, McNeil J. Protocol for a pilot randomised controlled clinical trial to compare the effectiveness of a graduated three layer straight tubular bandaging system when compared to a standard short stretch compression bandaging system in the management of people with venous ulceration: 3VSS2008. Trials 2010a;11(26):Available from http://www.trialsjournal.com/content/11/1/26 (accessed 25/08/2011).

Weller CD, Jolley D, McNeil J. Sub-bandage pressure difference of tubular form and short-stretch compression bandages; invivo randomised controlled trial. Wound Practice and Research 2010b;18(2):100-5.

\section{Additional references}

\section{AAWC 2010}

Association for the Advancement of Wound Care (AAWC). Association for the Advancement of Wound Care (AAWC) venous ulcer guideline. Available from http://aawconline.org/wpcontent/uploads/2011/04/AAWC-VU_Guideline.pdf (accessed December 2011) 2010.

\section{Altman 1991}

Altman DG. Practical statistics for medical research. London, UK: Chapman \& Hall, 1991.

\section{Altman 1997}

Altman DG, Bland JM. Statistics notes: units of analysis. BMJ 1997;314(7098):1874.

\section{Amsler 2009}

Amsler F, Willenberg T, Blättler W. In search of optimal compression therapy for venous leg ulcers: a meta-analysis of studies comparing diverse bandages with specifically designed stockings. Journal of Vascular Surgery 2009;50(3):668-74.

\section{Barwell 2004}

Barwell JR, Davies CE, Deacon J, Harvey K, Minor J, Sassano A, et al. Comparison of surgery and compression with compression alone in chronic venous ulceration (ESCHAR study): randomised controlled trial. Lancet 2004;363:1854-9.

\section{Belmont Pharmacy 2012}

Belmont Pharmacy. Product description: Bio Detralex Plus Capsules 60s. Available from http:// www.belmontpharmacy.com/deca90.html (accessed September 2012). 


\section{Bland 1998}

Bland JM, Altman DG. Survival probabilities (the Kaplan-Meier method). BMJ 1998;317:1572.

\section{Bland 2000}

Bland M. An introduction to medical statistics. 3rd Edition. Oxford, UK: Oxford University Press, 2000.

\section{BNF 2012}

British Medical Association and Royal Pharmaceutical Society of Great Britain. British National Formulary. British National Formulary available from http://www.medicinescomplete.com/ mc/bnf/current/ (accessed September 2012).. London, UK: BMJ Group and RPS Publishing, September 2012.

\section{Bosanquet 1992}

Bosanquet N. Costs of venous ulcers - from maintenance therapy to investment programs. Phlebology 1992;7:44-6.

\section{British Standards Institute1995}

British Standards Institute. Specification for the elastic properties of flat, non-adhesive, extensible fabric bandages. BS 7505:1995 1995

\section{Brown 2004}

Brown S, Cranny G. Prognostic factors of healing in venous leg ulcers (poster presentation). 2nd World Union of Wound Healing Societies' Meeting; 8-13 July, 2004; Paris, France. 2004.

\section{Callam 1985}

Callam MJ, Ruckley CV, Harper DR, Dale JJ. Chronic ulceration of the leg: Extent of the problem and provision of care. British Medical Journal 1985;290(6485):1855-6.

\section{Callam 1987}

Callam MJ, Ruckley CV, Dale JJ, Harper DR. Hazards of compression treatment of the leg: an estimate from Scottish surgeons. BMJ 1987;295:1382.

\section{Callam 1992a}

Callam M. Prevalence of chronic leg ulceration and severe chronic venous disease in western countries. Phlebology 1992; 7(Suppl 1):6-12.

\section{Cullen 2009}

Cullen GH, Phillips TJ. Clinician's perspectives on the treatment of venous leg ulceration. International Wound Journal 2009;6:367-78.

\section{Drew 2007}

Drew P, Posnett J, Rusling L on behalf of the Wound Care Audit Team. The cost of wound care for a local population in England. International Wound Journal 2007;4:149-55.

\section{Elashoff 1983}

Elashoff JD. Surviving proportional hazards. Hepatology 1983;3:1031-5.

\section{Ellison 2002}

Ellison DA, Hayes L, Lane C, Tracey A, McCollum CN. Evaluating the cost and efficacy of leg ulcer care provided in two large UK health authorities. Journal of Wound Care 2002;11(2):47-51.

\section{European Medicines Agency 2005}

Committee for Medicinal Products for Human Use (CHMP). Guideline on the choice of the non-inferiority margin. European Medicines Agency 2005; Vol. Available from http:// www.ema.europa.eu/docs/en_GB/document_library/ Scientific_guideline/2009/09/WC500003636.pdf (accessed August 2012):1-11.

\section{Feben 2003}

Feben $\mathrm{K}$. How effective is training in compression bandaging technique?. British Journal of Community Nursing 2003;8(2):80-4.

\section{Franks 1995}

Franks PJ, Moffatt CJ, Connolly M, Bosanquet N, Oldroyd MI, Greenhalgh RM, et al. Factors associated with healing leg ulceration with high compression. Age and Ageing 1995;24(5):407-10.

\section{Gilman 1990}

Gilman TH. Parameter for measurement of wound closure. Wounds: A Compendium of Clinical Research and Practice 1990;2(3):95-101.

\section{Graham 2003}

Graham ID, Harrison MB, Nelson EA, Lorimer K, Fisher A. Prevalence of lower-limb ulceration: a systematic review of prevalence studies. Advances in Skin and Wound Care 2003;16:305-16.

\section{Herber 2007}

Herber OR, Schnepp W, Rieger MA. A systematic review on the impact of leg ulceration on patients' quality of life. BioMed Central Health and Quality of Life Outcomes 2007;5:44. [DOI: doi:10.1186/1477-7525-5-44]

\section{Higgins 2002}

Higgins JPT, Thompson SG. Quantifying heterogeneity in a meta-analysis. Statistics in Medicine 2002;21:1539-58.

\section{Higgins 2003}

Higgins JPT, Thompson SG, Deeks JJ, Altman DG. Measuring inconsistency in meta-analyses. BMJ 2003;327(7414):557-60.

\section{Higgins 2011}

Higgins JPT, Altman DG, on behalf of the Cochrane Statistical Methods Group and the Cochrane Bias Methods Group (Editors). Chapter 8: Assessing risk of bias in included studies. In: Higgins JPT, Green S (editors). Cochrane Handbook for Systematic Reviews of Interventions Version 5.1.0 [updated March 2011]. The Cochrane Collaboration, 2011. Available from www.cochrane-handbook.org.

\section{Häfner 2001}

Häfner HM, Piche E, Jünger M. The ratio of working pressure to resting pressure under compression stockings: its significance 
for the improvement of venous perfusion in the legs. Phlebologie 2001;30(4):88-93.

\section{Kalbfleisch 2002}

Kalbfleisch JD, Prentice RL. The statistical analysis of failure time data. 2nd Edition. Hoboken, New Jersey, USA: Wiley, 2002.

\section{Lefebvre 2011}

Lefebvre C, Manheimer E, Glanville J, on behalf of the Cochrane Information Retrieval Methods Group. Chapter 6: Searching for studies. In: Higgins JPT, Green S (editors). Cochrane Handbook for Systematic Reviews of Interventions Version 5.1.0 [updated March 2011]. The Cochrane Collaboration, 2011. Available from www.cochrane-handbook.org.

\section{Lorimer 2003}

Lorimer KR, Harrison MB, Graham ID, Friedberg E, Davies B. Assessing venous ulcer population characteristics and practices in a home care community. Ostomy/Wound Management 2003;49(5):32-43.

\section{Margolis 2000}

Margolis DJ, Berlin JA, Strom BL. Which venous leg ulcers will heal with limb compression bandages?. American Journal of Medicine 2000;109(1):15-9.

\section{Margolis 2004}

Margolis DJ, Allen-Taylor L, Hoffstad O, Berlin JA. The accuracy of venous leg ulcer prognostic models in a wound care system. Wound Repair and Regeneration 2004;12(2):163-8.

\section{Moffatt 1995}

Moffatt C, Dorman M. Recurrence of leg ulcers within a community leg ulcer service. Journal of Wound Care 1995;4(2):56-62.

\section{Moffatt 2004}

Moffatt CJ, Franks PJ, Doherty DC, Martin R, Blewett R, Ross F. Prevalence of leg ulceration in a London population. Quarterly Journal of Medicine 2004;97:431-7.

\section{Moffatt 2007}

Moffatt C. Compression therapy in practice. Aberdeen, UK: Wounds UK Publishing, 2007.

\section{Moffatt 2009}

Moffatt C, Kommala D, Dourdin N, Choe Y. Venous leg ulcers: patient concordance with compression therapy and its impact on healing and prevention of recurrence. International Wound Journal 2009;6(5):386-93.

\section{Nelson 1995}

Nelson EA, Harper DR, Ruckley CV, Prescott RJ, Gibson B, Dale JJ. A randomised trial of single layer and multi-layer bandages in the treatment of chronic venous ulceration. Phlebology 1995;Suppl 1:915-6.

\section{Nelson 2007b}

Nelson EA. Evidence - recognising quality. 17th Conference of the European Wound Management Association; 2-4 May 2007; Glasgow, UK. 2007.

\section{Nelson 2011}

Nelson EA, Mani R, Thomas K, Vowden K. Intermittent pneumatic compression for treating venous leg ulcers. Cochrane Database of Systematic Reviews 2011, Issue 2. [DOI: 10.1002/14651858.CD001899.pub3]

\section{Noonan 1998}

Noonan L, Burge SM. Venous leg ulcers: is pain a problem?. Phlebology 1998;13:14-9.

\section{Palfreyman 1998}

Palfreyman SJ, Lochiel R, Michaels JA. A systematic review of compression therapy for venous leg ulcers. Vascular Medicine 1998;3(4):301-13.

\section{Partsch 2008b}

Partsch H, Clark M, Mosti G, Steinlechner E, Schuren J, Abel M, et al. Classification of compression bandages: practical aspects. Dermatologic Surgery 2008;34(5):600-9.

\section{Peduzzi 1996}

Peduzzi P, Concato J, Kemper E, Holford TR, Feinstein AR. A simulation study of the number of events per variable in logistic regression analysis. Journal of Clinical Epidemiology 1996;49:1373-9.

\section{Persoon 2004}

Persoon A, Heinen MM, van der Vleuten CJM, de Rooij MJ, van de Kerkhof PCM, van Achterberg T. Leg ulcers: a review of their impact on daily life. Journal of Clinical Nursing 2004;13:341-54.

\section{Posnett 2007}

Posnett J, Franks PJ. The costs of skin breakdown and ulceration in the UK. In: Pownall M editor(s). Skin Breakdown: The Silent Epidemic. Hull: Smith \& Nephew Foundation, 2007.

\section{Rabe 2008}

Rabe E, Partsch H, Junger M, Abel M, Achhammer I, Becker F, et al. Guidelines for clinical studies with compression devices in patients with venous disorders of the lower limb. European Journal of Vascular and Endovascular Surgery 2008;35(4):494-500.

\section{Ragnarson Tennvall 2005}

Ragnarson Tennvall G, Hjelmgren J. Annual costs of treatment for venous leg ulcers in Sweden and the United Kingdom. Wound Repair and Regeneration 2005;13:13-18.

\section{Royal College of Nursing 2006}

Royal College of Nursing. The nursing management of patients with venous leg ulcers: Recommendations. http://www.rcn.org.uk/_data/assets/ pdf_file/0003/107940/003020.pdf 2006.

\section{Schulz 2010}

Schulz KF, Altman DG, Moher D for the CONSORT Group. CONSORT 2010 Statement: updated guidelines for reporting parallel group randomised trials. Public Library of Science medicine 2010;7(3):e1000251. doi:10.1371/ journal.pmed.1000251. 


\section{SIGN 2010}

Scottish Intercollegiate Guidelines Network (SIGN). Management of chronic venous leg ulcers: a national clinical guideline. Available from http://www.sign.ac.uk/guidelines/ fulltext/120/index.html (accessed December 2011) 2010.

\section{SIGN 2012}

Scottish Intercollegiate Guidelines Network (SIGN). Search filters. Available from http://www.sign.ac.uk/methodology/ filters.html\#random (accessed 6 September 2012).

\section{Skene 1992}

Skene AI, Smith JM, Dore CJ, Charlett A, Lewis JD. Venous leg ulcers: a prognostic index to predict time to healing. $B M J$ 1992;305(6862):1119-21.

\section{Srinivasaiah 2007}

Srinivasaiah N, Dugdall H, Barrett S, Drew PJ. A point prevalence survey of wounds in north-east England. Journal of Wound Care 2007;16(10):413-9.

\section{Sterne 2008}

Sterne J, Jüni P, Moher D. Investigating and dealing with bias in systematic reviews. 16th Cochrane Colloquium. Evidence in the era of globalisation; 3-7 October 2008; Freiburg, Germany. 2008.

\section{Stewart 1993}

Stewart LA, Parmar MKB. Meta-analysis of the literature or of individual patient data: is there a difference?. Lancet 1993;341:418-22.

\section{Stewart 1995}

Stewart LA, Clarke MJ, on behalf of the Cochrane Working Group on Meta-analysis using individual patient data. Practical methodology of meta-analyses (overviews) using updated individual patient data. Statistics in Medicine 1995;14:2057-79.

\section{Stewart 2002}

Stewart LA, Tierney JF. To IPD or not to IPD? Advantages and disadvantages of systematic reviews using individual patient data. Evaluation and the Health Professions 2002;25(1):76-97.

\section{Stragen Chemical 2011}

Stragen Chemical SA. What is Predex TM?. Available from http:// www.predex.net/about_predex.php (accessed 13 May 2011).

\section{Thomas 1995}

Thomas S. Compression bandages. In: Cullum N, Roe BH editor(s). Leg Ulcers: nursing management. Harrow: Scutari, 1995.

\section{Vowden 2009a}

Vowden KR, Vowden P. A survey of wound care provision within one English health care district. Journal of Tissue Viability 2009;18:2-6.

\section{Vowden 2009b}

Vowden KR, Vowden P. The prevalence, management and outcome for patients with lower limb ulceration identified in a wound care survey within one English health care district. Journal of Tissue Viability 2009;18:13-9.

\section{Vowden 2009c}

Vowden K, Vowden P, Posnett J. The resource costs of wound care in Bradford and Airedale primary care trust in the UK. Journal of Wound Care 2009;18(3):93-102.

\section{References to other published versions of this review}

\section{Cullum 1997}

Cullum N, Fletcher AF, Semlyen A, Sheldon T. Compression therapy for venous leg ulcers. Quality in Health Care 1997;6(4):226-31.

\section{EHCB 1997}

Effective Health Care. Compression therapy for venous leg ulcers. Compression therapy for venous leg ulcers. Vol. 3(4), London: FT Healthcare, 1997.

\section{Fletcher 1997}

Fletcher A, Cullum N, Sheldon T. A systematic review of compression treatment for venous leg ulcers. $B M J$ 1997;315(7108):576-80.

\section{O'Meara 2009}

O'Meara S, Tierney J, Cullum N, Bland JM, Franks PJ, Mole T, et al. Four layer bandage compared with SSB for venous leg ulcers: systematic review and meta-analysis of randomised controlled trials with data from individual patients. BMJ 2009 Apr 17;338:doi: 10.1136/bmj.b1344.

* Indicates the major publication for the study

\section{CHARACTERISTICS OF STUDIES}

Characteristics of included studies [ordered by study ID]

\section{Blecken 2005}

\section{Methods}

RCT (within individual randomisation, no other details about method of randomisation). Trial conducted in USA, type of setting not described.
Recruited 12 patients with post-thrombotic bilateral venous leg ulcers ( 7 men, 5 women). All had history of DVT.

Inclusion criterion: $\mathrm{ABPI} \geq 1.00$. 
Exclusion criteria: chronic or acute systemic disease; and impaired mobility secondary to rheumatoid arthritis.

Mean patient age 61 years; range 45-82 (breakdown/group not reported).

At baseline, patients had had active ulcers for 1-6 years.

Mean \pm SE baseline ulcer area ( $\left.\mathrm{cm}^{2}\right)$ : Group 1: $48.98 \pm 14.13$; Group 2: $50.08 \pm 18.30$ (95\% Cl of difference Group 1 minus Group 2: -27.25-25.07).

Interventions

All patients: prior to bandage application, ulcers cleansed with neutral soap and water and skin lubricated with lanolin. Compression reapplied every $72 \mathrm{~h}$.

Group 1: adjustable-compression boot system consisting of: fine mesh paraffin-impregnated gauze primary dressing (Aquafor); single layer of sterile absorbent gauze; $1 \mathrm{~cm}$-thick felt pad cushion; surgical cotton stockinette; non-elastic compression garment comprising a series of individually adjustable Velcro bands $5.1 \mathrm{~cm}$ wide extending from ankle to knee (CircAid); and elastic anklet (Medi) applied from base of toes to $5 \mathrm{~cm}$ above the malleolus ( $\mathrm{n}=12$ limbs).

Group 2: 4-layer bandage (4LB) comprising: fine mesh paraffin-impregnated gauze primary dressing (Aquafor); single layer of sterile absorbent gauze; $1 \mathrm{~cm}$-thick felt pad overlapping at least $3 \mathrm{~cm}$ of ulcer area; thick gauze bandage (Kerlix); and $15 \mathrm{~cm}$ wide elastic bandage ( $\mathrm{n}=12 \mathrm{limbs}$ ).

Outcomes Number (\%) limbs with complete healing at 12 weeks: Group 1: 4/12 (33\%); Group 2: 4/12 (33\%). Note: the same 4 individuals healed in each group.

Mean \pm SE ulcer area reduction rate ( $\mathrm{cm}^{2}$ per week): Group 1: $2.93 \pm 0.60$; Group 2: $2.30 \pm 0.70(95 \% \mathrm{Cl}$ of difference Group 1 minus Group 2: 0.05-1.21), $P=0.037$ (paired t-test).

HR for area reduction rate: $0.56(95 \% \mathrm{Cl} 0.33-0.96), \mathrm{P}=0.017$ (indicating faster healing rate in Group 1). The authors reported that patient age and sex were not associated with reduction rate, but statistics for covariates were not shown.

Mean \pm SE patient satisfaction score, assessed with scoring sheet at 12 weeks ( $1=$ not satisfied; $2=$ moderately satisfied; 3 = very satisfied): Group 1: $2.92 \pm 0.08$; Group 2: $2.58 \pm 0.15$ (95\% Cl of difference Group 1 minus Group 2: -0.08-0.75), $\mathrm{P}=0.104$.

Notes Ulcer area assessed at baseline then every 4 weeks by direct grid tracing combined with digital imaging. 4LB system was not the traditional one. No withdrawals. Skill of care provider not explained. HR for area reduction rate difficult to interpret as outcome variable was continuous rather than time-to-event.

\section{Risk of bias}

\begin{tabular}{lll}
\hline Bias & Authors' judgement & Support for judgement \\
\hline $\begin{array}{l}\text { Random sequence genera- } \\
\text { tion (selection bias) }\end{array}$ & Unclear risk & "This was a randomised study .... \\
\hline $\begin{array}{l}\text { Allocation concealment } \\
\text { (selection bias) }\end{array}$ & Unclear risk & No details provided. \\
\hline $\begin{array}{l}\text { Incomplete outcome data } \\
\text { (attrition bias) } \\
\text { All outcomes }\end{array}$ & Low risk & 12 people recruited and all appear in results (individual patient data). \\
\hline $\begin{array}{l}\text { Blinded outcome assess- } \\
\text { ment (healing) }\end{array}$ & Unclear risk & No details provided. \\
\hline $\begin{array}{l}\text { Baseline comparability } \\
\text { Unclear risk }\end{array}$ & $\begin{array}{l}\text { Mean values reported for baseline ulcer area, and so difficult to judge compa- } \\
\text { rability; no ulcer duration data presented. }\end{array}$ \\
\hline
\end{tabular}


Methods

Single-centred RCT. Randomisation stratified according to presence/absence of deep venous reflux (assessed using duplex ultrasound) using blocks of 4 and 6 respectively. Setting: an outpatient clinic in Argentina. A statistical power calculation was reported, but methods used for estimation not clear.

Participants 60 patients recruited by referral from outpatient clinics of general hospitals.

Inclusion criteria: venous leg ulcer (diagnosed clinically and by duplex ultrasound) with surface area $3-50 \mathrm{~cm}^{2}$, present for at least 2 months and not treated with compression during previous 2 months.

Exclusion criteria: malignancy, respiratory or cardiac failure, liver disease, kidney disease, mental illness, severe peripheral neuropathy, peripheral arterial disease and osteoarthritis of hips or knees.

Baseline data from 55 patients analysed:

Number of male:female patients: Group 1: 19:9; Group 2: 17:10.

Mean \pm SD (median) patient age in years: Group 1: $62.1 \pm 9.9$ (62.0); Group 2: $61.4 \pm 13.1$ (64.0).

Number (\%) patients with recurrent ulceration: Group 1: 12/28 (43\%); Group 2: 15/27 (56\%).

Mean \pm SD (median) baseline ulcer duration in months: Group 1: $26.9 \pm 44.7$ (11.5); Group 2: $26.7 \pm 29.7$ (12.0).

Mean \pm SD (median) baseline ulcer surface area in $\mathrm{cm}^{2}$ : Group 1: $13.1 \pm 14.5$ (5.0); Group 2: $12.2 \pm 12.8$ (6.0).

Mean \pm SD (range) score for pain in ulcer area/lower leg assessed using Likert scale with range 0-100 $(0=$ no pain, 100 = maximum pain): Group 1: $44.2 \pm 33.1$ (0-100); Group 2: $45.8 \pm 26.4(0-100)$.

Mean \pm SD quality of life score assessed using the Spanish version of Chronic Venous Insufficiency Quality of Life (CIVIQ) questionnaire with range 20-100 (20 = best quality of life, $100=$ worst quality of life): Group 1: $53 \pm 18$; Group 2: $53 \pm 15$.

Interventions

All patients: at each visit ulcers were cleaned with Ringer's lactate solution and debrided using wound lavage (Jetox-ND, TavTech Ltd, Israel). Surrounding skin treated with gentian violet, moisturised and the ulcer covered with paraffin gauze (Bactigras, Smith \& Nephew, UK). In order to provide additional compression, a rubber-foam pad ( $5 \mathrm{~cm}$ thick) was cut to fit the space above insufficient perforating and/or large communicating veins identified by duplex ultrasound. Compression then applied and left in situ day and night. Ulcer care performed by experienced staff at the clinic. Both compression devices changed weekly.

Group 1: graduated compression stocking with open toe (prototype stocking provided by Sigvaris Inc.) with fibre consisting of $92 \%$ nylon and $8 \%$ Lycra and available in 4 ankle sizes. Applied over a gauze bandage using a donning device $(n=28$ patients).

Group 2: short-stretch bandage (SSB), consisting of 3 "short-stretch slings" (Tesadur, $40 \%$ elongation, $10 \mathrm{~cm}$ width, $7 \mathrm{~m}$ length, Filmar, Italy). Method of application not stated ( $\mathrm{n}=27$ patients).

Outcomes Number (\%) patients with complete healing at 90 days: Group 1: 10/32 (31\%); Group 2: 13/28 (46\%).

Logistic regression suggested the following as significant (at $5 \%$ level), independent predictors of nonhealing at 90 days: lower BMI and larger baseline ulcer surface area.

Number (\%) patients with complete healing at 180 days: Group 1: 14/32 (44\%); Group 2: 18/28 (64\%).

Logistic regression suggested the following as significant (at $5 \%$ level), independent predictors of nonhealing at 180 days: older patient age, larger baseline ulcer surface area, longer baseline ulcer duration and recurrent ulceration.

Median days to healing: Group 1: 56; Group 2: 60.

Cox regression did not detect any significant (at 5\% level), independent predictors of delayed healing. 
Brizzio 2010 (Continued)

Mean \pm SD (range) pain score at 13 weeks: Group 1: $17.7 \pm 18.8$ (0-63), $\mathrm{P}<0.001$ for within-group change from baseline; Group 2: $11.1 \pm 15.6$ (0-63), $\mathrm{P}<0.01$ for within-group change from baseline. $\mathrm{P}$ value for between-group difference not reported.

Mean \pm SD quality of life score (CIVIQ) at 35 days: Group 1: $44 \pm 16$; Group 2: $44 \pm 19, P=0.944$ for between-group difference.

Mean \pm SD quality of life score (CIVIQ) at 90 days in patients with healed ulcers: Group 1: $44 \pm 18$; Group 2: $45 \pm 17, P=0.825$ for between-group difference.

Mean \pm SD quality of life score (CIVIQ) at final assessment in patients with healed ulcers: Group 1: $39 \pm$ 18; Group 2: $30 \pm 17, \mathrm{P}=0.109$ for between-group difference.

Linear regression did not detect any significant (at $5 \%$ level), independent predictors of quality of life score at 35 days or final assessment.

Notes Ulcers assessed weekly using photography and measurement of surface area using transparent foil.

Compression stockings were designed to exert pressure above the ankle of $15-20 \mathrm{~mm} \mathrm{Hg}$.

Pressure measurements were taken from a sub-group of randomly selected patients (number not stated). Immediately after application, the mean \pm SD pressure in $\mathrm{mm} \mathrm{Hg}$ exerted by compression devices worn over dressings and pads was: Group 1: $28.6 \pm 9.2$; Group 2: $48.6 \pm 15.1$.

The CIVIQ questionnaire included 4 dimensions of quality of life: pain, physical, social and psychological. Further analysis of patients with both healed and unhealed ulcers in relation to individual dimensions of CIVIQ scores at 90 days suggested that pain decreased by $50 \%$ with treatment regardless of healing. Physical, social and psychological dimensions showed significant improvement over time only in patients with healed ulcers. Total score showed significant improvement over time in both healed and unhealed patients.

Numbers of patients (with reasons) who withdrew before completion: Group 1: 4 patients (1 sudden death deemed unrelated to venous disease or its treatment; 1 did not attend clinic after 11 weeks; 2 had systemic infection requiring hospital treatment). Group 2: 1 patient (rapid deterioration).

\section{Risk of bias}

\section{Bias \\ Authors' judgement Support for judgement}

Random sequence genera- Unclear risk tion (selection bias)

"Leg compression was carried out randomly with either stockings or ban-
dages".

"Patients with and without deep venous reflux were randomised separately using sealed envelopes in blocks of four and six, respectively".
"Patients with and without deep venous reflux were randomised separately using sealed envelopes in blocks of four and six, respectively".

It was not clear whether envelopes were opaque and/or opened in sequential order.

\begin{tabular}{lll}
\hline $\begin{array}{l}\text { Incomplete outcome data } \\
\text { (attrition bias) } \\
\text { All outcomes }\end{array}$ & High risk & $\begin{array}{l}60 \text { patients randomised; } 55 \text { patients analysed. Withdrawal rate higher in group } \\
\text { receiving stockings (12.5\% vs 3.6\%). }\end{array}$ \\
\hline $\begin{array}{l}\text { Blinded outcome assess- } \\
\text { ment (healing) }\end{array}$ & Unclear risk & $\begin{array}{l}\text { "This randomised open-label trial was performed at a specialized outpatient } \\
\text { clinic...". }\end{array}$ \\
& It was not clear whether outcome assessment was blinded.
\end{tabular}

Baseline comparability Low risk Groups appeared comparable at baseline.


Callam 1992b

\begin{tabular}{ll}
\hline Methods & RCT, factorial design. Setting was 2 hospital outpatient clinics in Scotland, UK. \\
\hline Participants & 132 patients recruited from those attending hospital-based leg ulcer clinics in 2 hospitals in Scotland, \\
UK. & Inclusion criteria: not stated. \\
& Exclusion criteria: ABPI < 0.8, diabetes, sero-positive rheumatoid arthritis, lived too far away, refused \\
& consent. \\
& Number of male:female patients: Group 1: 33:32; Group 2: $26: 41$. \\
& Mean patient age in years: Group 1: $62 ;$ Group 2: 65. \\
& Mean \pm SD baseline ulcer area in cm²: Group 1: $8.2 \pm 12.9 ;$ Group 2: $11.0 \pm 15.9$. \\
& Number of patients with baseline ulcer duration <6 months; 6-11 months; $1-2$ years; $\geq 3$ years: Group 1: \\
& $27 ; 19 ; 13 ; 6 ;$ Group 2: $37 ; 17 ; 12 ; 1$. \\
& Number (\%) patients walking with difficulty: Group 1: 15/65 (23\%); Group 2: $17 / 67$ (25\%).
\end{tabular}

Interventions

Group 1: 3-component compression system consisting of: orthopaedic wool (Soffban Natural), elastic bandage (Tensopress), and cotton-elastic graduated compression tubular support bandage (Tensoshape) ( $n=65$ patients).

Group 2: 3-component compression system consisting of: orthopaedic wool (Soffban Natural), nonelastic cotton-elastic bandage (Elastocrepe), and non-elastic cotton-Lycra cohesive bandage (Tensoplus Forte) ( $n=67$ patients).

All bandages applied by experienced research nurses using a spiral technique.

Patients were further randomised within the above groups to a knitted viscose dressing (Tricotex) or a hydrocellular polyurethane foam dressing (Allevyn).

Outcomes Number (\%) patients with complete healing at 12 weeks: Group 1: 35/65 (54\%); Group 2: 19/67 (28\%), P $=0.01$, Cox proportional hazards model.

No statistically significant interaction detected between dressings and bandages $(P=0.87$, Cox proportional hazards model).

Mean \pm SD number of bandage changes during the 12-week trial period: Group 1: $11.7 \pm 6.7$; Group 2: $12.3 \pm 6.5$ (reported as not significant, but $P$ value not shown).

Trial authors reported that: "two patients in each group sustained bandage damage although this was minor in all cases".

Proportion of patients reporting ulcer pain at all clinic visits: Group 1: 29\%; Group 2: $48 \%(P=0.03$, Wilcoxon two-sample test).

Number (\%) patients who withdrew (> 1 reason/patient): Group 1: 8/65 (12\%) (2 sensitivity; 3 exudate; 7 deterioration of ulcer; 1 social; 3 other - included bandage slippage and patient intolerance); Group 2: 20/67 (30\%) (8 sensitivity; 10 exudate; 17 deterioration of ulcer; 1 social; 7 other - included bandage slippage and patient intolerance), $P=0.025$, chi-squared test, for difference between groups in proportions of patients who withdrew.

Notes

Ulcer area measured using transparency tracing and computerised planimetry at baseline, then every 4 weeks. Possible imbalance in baseline variables: larger ulcers in Group 2, but more ulcers of longer duration in Group 1:

\section{Risk of bias}

Bias Authors' judgement Support for judgement

Random sequence genera- Unclear risk $\quad$ No details provided.
tion (selection bias)


Callam 1992b (Continued)

\begin{tabular}{lll}
$\begin{array}{l}\text { Allocation concealment } \\
\text { (selection bias) }\end{array}$ & Unclear risk & No details provided. \\
\hline $\begin{array}{l}\text { Incomplete outcome data } \\
\text { (attrition bias) }\end{array}$ & Low risk & All patients randomised appear in the analysis. \\
All outcomes &
\end{tabular}

\begin{tabular}{lll}
\hline $\begin{array}{l}\text { Blinded outcome assess- } \\
\text { ment (healing) }\end{array}$ & Unclear risk & Not stated. \\
\hline Baseline comparability & Unclear risk & $\begin{array}{l}\text { Difficult to assess from data presented. Mean ulcer area slightly greater in } \\
\text { Group 2, but slightly more ulcers of longer duration in Group 1. }\end{array}$ \\
\hline
\end{tabular}

Charles 1991

\begin{tabular}{ll}
\hline Methods & RCT, no details of methods. Outpatient setting in inner London, UK. \\
\hline Participants & 53 patients with venous leg ulcers. \\
& Inclusion criterion: ABPI > 0.8. \\
& Mean (range) patient age in years: Group 1: 78 (55-99); Group 2: 75 (37-91). \\
& Mean (range) baseline ulcer area in cm²: Group 1: 12.0 (1.5-52.0); Group 2: 15.0 (1.0-88.0). \\
& Mean (range) baseline ulcer duration in months: Group 1: 32 (4-336); Group 2: 25 (4-120). \\
\hline
\end{tabular}

Interventions

Group 1: compression system applied by project nurse. Application consisted of: primary dressing (not defined); foam padding covered with gauze; further padding (Cellona) to bony prominences, as required; SSB (Rosidal K) applied spirally with $50 \%$ overlap and no more than $90 \%$ stretch from toes to knee. 1 bandage $(10 \mathrm{~cm} \times 5 \mathrm{~m})$ used except for 5 patients with a higher degree of mobility who had 2 bandages. Bandages changed 1-3 times/week; washed by the patient and reused ( $n=27$ patients). Group 2: continuation of usual care by district nurse (no patients received SSB) ( $n=26$ patients).

Proportion of patients with complete healing at 3 months: Group 1: 71\%; Group 2: 25\%. Authors report-
ed that between-group difference was statistically significant (chi-squared test) but did not report P
value. NB: raw data for number of patients experiencing complete healing not provided in the paper,
and review authors have not extrapolated these values from the reported percentages, as group de-
nominators were unclear.
Proportion of patients with increase in ulcer area during the 3-month trial: Group 1: 0\%; Group 2: $21 \%$.
Number (\%) patients who withdrew during the trial (reasons): Group 1: $3 / 30$ (10\%) (2 refused treat-
ment, 1 referred for surgery); Group 2: $3 / 29$ (10\%) ( 3 admitted to hospital for leg ulcer treatment). NB: it
was unclear whether these 6 patients were included in the 53 patients described above.

\section{Risk of bias}

\begin{tabular}{lll}
\hline Bias & Authors' judgement & Support for judgement \\
\hline $\begin{array}{l}\text { Random sequence genera- } \\
\text { tion (selection bias) }\end{array}$ & Unclear risk & $\begin{array}{l}\text { "Patients ... were randomly divided into a control and an experimental } \\
\text { group". }\end{array}$ \\
\hline $\begin{array}{l}\text { Allocation concealment } \\
\text { (selection bias) }\end{array}$ & Unclear risk & $\begin{array}{l}\text { "Patients... were randomly divided into a control and an experimental } \\
\text { group". }\end{array}$ \\
\hline
\end{tabular}


Charles 1991 (Continued)

Incomplete outcome data Unclear risk 6 people withdrew from treatment, but unclear whether they were included in (attrition bias) the analysis; only \% healed reported for outcome - no raw numbers.

All outcomes

\begin{tabular}{|c|c|c|}
\hline $\begin{array}{l}\text { Blinded outcome assess- } \\
\text { ment (healing) }\end{array}$ & Unclear risk & $\begin{array}{l}\text { No detail regarding outcome assessment, however, implied that the treating } \\
\text { nurses assessed outcome. }\end{array}$ \\
\hline
\end{tabular}

\begin{tabular}{ll}
\hline Baseline comparability $\quad$ Unclear risk & $\begin{array}{l}\text { Only mean data presented, but possible imbalance: larger ulcers in Group 2; } \\
\text { ulcers of longer duration in Group } 1 .\end{array}$
\end{tabular}

\section{Colgan 1995}

Methods RCT (single-centred). Outpatient setting in Ireland. Outcome assessment was non-blind.

Participants
Inclusion criteria: diagnosis of venous aetiology; ulcer size $>1 \mathrm{~cm}^{2}$.
Exclusion criterion: arterial disease (no definition provided).
Number of male:female patients: Group 1: 4:6; Group 2: 2:8; Group 3: 2:8.
Mean patient age in years: Group 1: 65.5; Group 2: 67.5; Group 3: 56.0.
Median (mean) baseline ulcer area in $\mathrm{cm}^{2}:$ Group 1: 9 (48.5); Group 2: 7 (27.5); Group 3: 20 (42.8).
Median (mean) baseline ulcer duration in months: Group 1: 24 (66.5); Group 2: 10 (9.3); Group 3: 12
(53.5).

Interventions

Group 1: modified Unna's boot, a compression system with 4 components: paste bandage; cotton crepe bandage (Elastocrepe); elastic adhesive bandage (Elastoplast); class II compression sock) ( $\mathrm{n}=10$ patients). Group 2: 4LB (Profore) ( $\mathrm{n}=10$ patients).

Group 3: polyurethane foam dressing (Lyofoam dressing) plus elastic bandage (Setopress) ( $\mathrm{n}=10 \mathrm{pa}-$ tients).

All patients: treatment delivered by clinic nurse. Treatment duration $=12$ weeks.

Outcomes Number (\%) patients with complete healing at 12 weeks: Group 1: 7/10 (70\%); Group 2: 6/10 (60\%); Group 3: 2/10 (20\%) (statistical tests not reported).

Number (\%) patients who withdrew (reasons): Group 1: 1/10 (10\%) (allergy); Group 2: 0/10 (0\%); Group 3: $3 / 10(30 \%)$ (3 inability to tolerate bandage).

No cases of ulcer recurrence in any group during the 6-month follow-up period that followed completion of the 12-week treatment period.

Costs of bandages were calculated, but not did not include nursing time, due to wide variation in services.

Average (range) cost/patient/12 weeks: Group 1: IEP 66.24 (18.14-108.84); Group 2: IEP 82.54

(27.94-177.20); Group 3: IEP 58.33 (19.11-83.24).

Notes

Risk of bias

\begin{tabular}{lll}
\hline Bias & Authors' judgement & Support for judgement \\
\hline $\begin{array}{l}\text { Random sequence genera- } \\
\text { tion (selection bias) }\end{array}$ & Unclear risk & "We undertook a prospective randomised study ...". \\
\hline $\begin{array}{l}\text { Allocation concealment } \\
\text { (selection bias) }\end{array}$ & Unclear risk & No details provided. \\
\hline
\end{tabular}


Colgan 1995 (Continued) Incomplete outcome data Low risk $\quad 30$ patients randomised and 30 patients analysed. (attrition bias)

All outcomes

$\begin{aligned} & \text { Blinded outcome assess- } \\ & \text { ment (healing) }\end{aligned}$ High risk $\quad$ Author correspondence: ". . . assessor was not blinded".

Baseline comparability High risk Initial ulcer size larger in Group 3; ulcer duration greater in Group 1.

\section{Cordts 1992}

Methods $\quad$ RCT (no details about methods). Set in an out-patient clinic in Boston, USA.

\section{Participants}

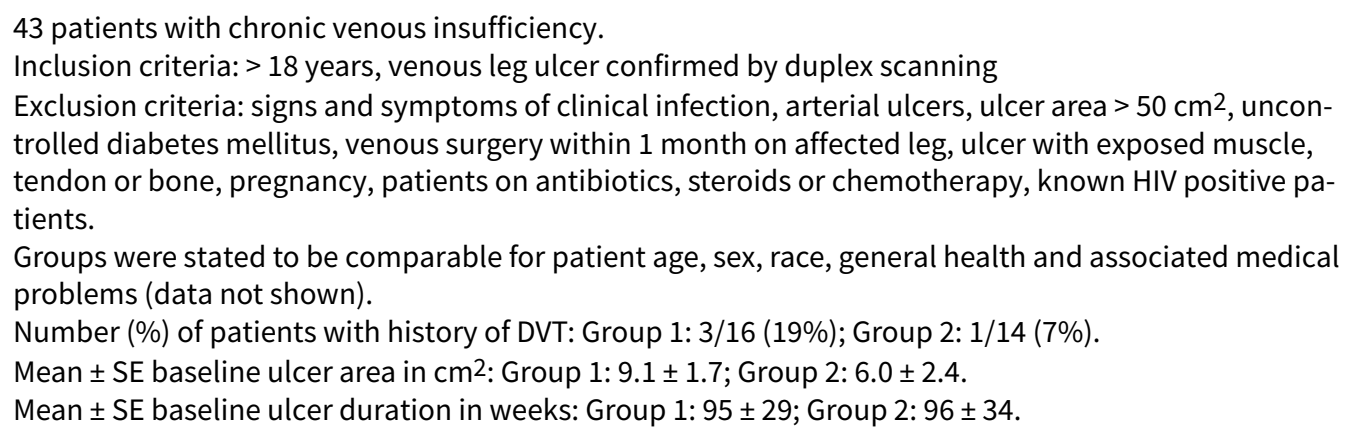

Mean \pm SE healing rate in $\mathrm{cm}^{2}$ per week adjusted for baseline ulcer perimeter in $\mathrm{cm}$ (i.e. healing rate divided by baseline ulcer perimeter): Group 1: $0.049 \pm 0.007$; Group 2: $0.0201 \pm 0.017(P=0.11$, Student's ttest).

Mean \pm SE pain score based on 1-10 scale where $0=$ no pain: Group 1: $1.0 \pm 0.16$; Group 2: $1.0 \pm 0.21$ (authors reported no significant difference, but did not show $P$ value).

Number (\%) of patients with adverse events not requiring withdrawal from treatment (description): Group 1: 2/16 (13\%) (1 necrosis at ulcer edge, 1 wound infection); Group 2: 3/14 (21\%) (all had wound infection).

Number (\%) of patients who withdrew from the trial: Group 1: 7/16 (44\%); Group 2: 6/14 (43\%). All withdrawals were because of failure to attend clinic.

Notes ceptance of bandage higher for Duoderm than Unna's boot.

\section{Risk of bias}


Cordts 1992 (Continued)

\begin{tabular}{|c|c|c|}
\hline Bias & Authors' judgement & Support for judgement \\
\hline $\begin{array}{l}\text { Random sequence genera- } \\
\text { tion (selection bias) }\end{array}$ & Unclear risk & "Patients were randomly assigned to treatment ...". \\
\hline $\begin{array}{l}\text { Allocation concealment } \\
\text { (selection bias) }\end{array}$ & Unclear risk & "Patients were randomly assigned to treatment ...". \\
\hline $\begin{array}{l}\text { Incomplete outcome data } \\
\text { (attrition bias) } \\
\text { All outcomes }\end{array}$ & High risk & $\begin{array}{l}43 \text { people randomised; analysis of only } 30 \text { people. Withdrawal rates similar in } \\
\text { both groups; reason for each was non-attendance at clinic. }\end{array}$ \\
\hline $\begin{array}{l}\text { Blinded outcome assess- } \\
\text { ment (healing) }\end{array}$ & Unclear risk & Not stated. \\
\hline Baseline comparability & Unclear risk & Mean (not median) ulcer areas given and larger in Group 1; durations similar. \\
\hline
\end{tabular}

\section{Danielsen 1998}

Methods $\quad$ RCT (randomisation stratified by baseline ulcer area, larger or smaller than $20 \mathrm{~cm}^{2}$ ). Set in a hospital
outpatient clinic in Copenhagen, Denmark.
Participants
43 patients were randomised.
Inclusion criteria: lipodermatosclerosis, leg ulcers and incompetent veins demonstrated by Doppler and/or clinical examination.
Exclusion criteria: significant arterial insufficiency (systolic blood pressure in 1st toe $<60 \mathrm{mmHg}$ or $\mathrm{ABPI}<0.9$ ), immunological aetiology of ulcer, diabetes, uncompensated heart disease, inability to walk unassisted.
Number of male:female patients (of 40 patients included in authors' analyses): Group 1: 12:9; Group 2:
8:11.
Median (range) patient age in years: Group 1: 72 (38-85); Group 2: 71 (37-90).
Mean [median] (range) baseline ulcer area in $\mathrm{cm}^{2}$ : Group 1: 19.7 [2.4] (0.3-124.5); Group 2: 16.5 [6.3]
(0.4-66.1).
Mean [median] (range) baseline ulcer duration in months: Group 1: 22.2 [12] (2-120); Group 2: 27.8 [15] $(2-84)$. press) applied in a spiral with 50\% overlap and approximately $86 \%$ extension. Usually 1 bandage used (3.5 m unstretched). Bandage changed every 1-7 days, according to wound exudate (left unchanged for as long as possible). All bandages applied by study nurse $(n=23$ patients).

Group 2: lower leg padded with gauze then non-adhesive, compressive SSB (Comprilan) applied in a spiral with $50 \%$ overlap, using similar tension to that in long stretch bandage. Usually $1 \frac{1}{2}$ SSBs were used (total unstretched length $4.5 \mathrm{~m}$ ). Bandages changed every 1-2 days; usually applied by community nurse ( $n=20$ patients).

All patients: hydrocolloid primary dressing (Comfeel) used, if possible. Patients with large ulcers, or maceration of the surrounding skin, treated with an non-antibacterial ointment/gel. When local infection was suspected, used mupirocin, silver sulphadiazine cream (Flamazine) or cadexomer iodine (Iodosorb). Systemic antibiotics given for cellulitis. Eczema of peri-ulcer skin treated with a steroid ointment. Patients continued with randomised bandage system after healing.

Outcomes

NB: the analyses of complete healing, incidence of cellulitis and withdrawals are as calculated by the review authors, according to intention-to-treat (complete case analysis). All other analyses are as reported by the trial authors, and are based on 40 patients overall (excluding 3 patients who were ineligible, Group 1: $n=21$ and Group 2: $n=19$ ). 
Danielsen 1998 (Continued)

Number (\%) patients with complete healing at 1 month: Group 1: 4/23 (17\%); Group 2: 1/20 (5\%).

Number (\%) patients with complete healing at 6 months: Group 1: 9/23 (39\%); Group 2: 5/20 (25\%).

Number (\%) patients with complete healing at 1 year: Group 1: 12/23 (52\%); Group 2:3/20 (15\%).

Kaplan-Meier estimate of the proportions of patients healed at 1 year: Group 1: 81\%; Group 2: 31\% (P=

$0.03, \log$ rank test).

Mean [median] (range) relative ulcer area at 12 months: Group 1: 0.25 [0] (0-3.11); Group 2: 0.95 [0.77]

(0-4.04) $(P<0.01$ for between group difference, Mann-Whitney test).

Number of patients who developed cellulitis: Group 1: 7/23 (30\%); Group 2: 8/20 (40\%).

Number of patients using: hydrocolloid; mupirocin; silver sulphadiazine; cadexomer iodine: Group 1: 6; $5 ; 3 ; 1 ;$ Group $2: 3 ; 2 ; 5 ; 2$.

Number (\%) of patients who withdrew during trial period (reasons): Group 1: 7/23 (30\%) (2 ineligible; 2 preferred compression stockings post-healing; 2 preferred other treatment; 1 knee pain/swelling because of bandage); Group 2: 10/20 (50\%) (1 ineligible; 1 preferred compression stockings post-healing; 3 preferred other treatment; 3 had poor compliance; 1 changed address; 1 died).

Notes

Ulcer area measured using transparency tracing and planimetry (instrument not stated) at baseline, 1 , 6 and 12 months. The authors stated that values for the total area of ulceration on the reference limb were studied.

Ankle sub-bandage pressure measured using an Oxford pressure monitor. Group 1: maintained mean pressure of $40 \mathrm{mmHg}$ at 1 week; Group 2: decreased mean pressure by $10 \mathrm{mmHg}$ during first $24 \mathrm{~h}$. The between-group differences at $2 \mathrm{~h}$ and $24 \mathrm{~h}$ was significant $(\mathrm{P}<0.001$ and $\mathrm{P}<0.017$ respectively).

This trial assessed incidence of healing and also maintenance of healing. Ulcers could have healed and recurred before the assessment points. It appears that 2 ulcers recurred after the 6-month assessment in Group 2.

Use of a variety of primary dressings and topical agents could have confounded the treatment effect.

\section{Risk of bias}

\begin{tabular}{|c|c|c|}
\hline Bias & Authors' judgement & Support for judgement \\
\hline $\begin{array}{l}\text { Random sequence genera- } \\
\text { tion (selection bias) }\end{array}$ & Unclear risk & ". . p patients were randomised to receive treatment ...". \\
\hline $\begin{array}{l}\text { Allocation concealment } \\
\text { (selection bias) }\end{array}$ & Unclear risk & $\begin{array}{l}\text { The authors reported that "randomisation was blind" but did not provide any } \\
\text { other details. }\end{array}$ \\
\hline $\begin{array}{l}\text { Incomplete outcome data } \\
\text { (attrition bias) } \\
\text { All outcomes }\end{array}$ & Low risk & $\begin{array}{l}3 \text { patients excluded from analysis by trial author, as deemed ineligible. These } \\
\text { were re-instated in denominators for the outcome of complete healing by the } \\
\text { review authors. }\end{array}$ \\
\hline $\begin{array}{l}\text { Blinded outcome assess- } \\
\text { ment (healing) }\end{array}$ & Unclear risk & No detail. \\
\hline Baseline comparability & High risk & Baseline median ulcer area and duration greater in Group 2: \\
\hline
\end{tabular}

\section{DePalma 1999}

\begin{tabular}{ll}
\hline Methods & RCT (multicentred, method of randomisation not stated). Setting: outpatients, USA. \\
\hline Participants & 38 outpatients recruited from 6 study centres. \\
& Inclusion criteria: patient age $\geq 18$ years; unilateral venous leg ulcer diagnosed by duplex examination. \\
\hline
\end{tabular}


DePalma 1999 (Continued)

Exclusion criteria: ulcers of non-venous or mixed aetiology; ulcer diameter $>5 \mathrm{~cm}$; severe arterial, metabolic or neuropathic disease; not expected to heal with conservative treatment; poor general health; using medications inhibiting wound healing; acute deep venous thrombosis within last 3 months; venous surgery within the last month; allergy to study materials; pregnant; likely to be noncompliant; deemed by investigators to be better treated by methods other than those used in the study.

Mean \pm SD patient age (years): Group 1: $63.95 \pm 9.73$; Group 2: $58.15 \pm 9.60$.

Mean \pm SD baseline ulcer area $\left(\mathrm{cm}^{2}\right)$ : Group 1: $3.59 \pm 3.54$; Group 2: $3.28 \pm 4.08$.

Mean \pm SD baseline ulcer duration (months): Group 1: $27.42 \pm 54.72$; Group 2: $12.28 \pm 14.54$.

Number of patients with chronic deep venous obstruction: Group 1: 4; Group 2: 5.

Interventions

All patients: ulcers cleansed and debrided (no further details given), dressed with paraffin-impregnated gauze (Adaptic) covered by $4 \times 4$ inch gauze pad (Curity), retained with a conforming gauze wrap (Kling).

Group 1: Unna's Boot consisting of zinc oxide, glycerin and gelatin-impregnated $10 \mathrm{~cm} \times 9 \mathrm{~m}$ roller gauze bandage (Medicopaste) covered by an elastic Ace type bandage $(n=19)$.

Group 2: Thera-Boot - a device consisting of a series of interlocking, non-elastic bands encircling the leg and held in place by hook and loop fasteners, plus a foot piece made of very low stretch bands. Patients adjusted the straps as necessary in order to maintain compression between clinic visits $(n=19)$.

Outcomes

Patients followed-up until healing, or for 12 weeks, and were seen as often as the investigator felt was appropriate. Ulcer area measured using transparency tracing.

Mean \pm SD area healing rate $\left(\mathrm{cm}^{2} /\right.$ day): Group 1: $0.0239 \pm 0.0534$; Group 2: $0.0433 \pm 0.0910, P=0.27$.

Mean \pm SD area healing rate (\%/day): Group 1: $1.0493 \pm 1.5583$; Group 2: $2.0357 \pm 1.9520, P=0.56$

Mean \pm SD linear healing rate $(\mathrm{cm} / \text { day })^{\star}:$ Group 1: $0.0060 \pm 0.0092 ;$ Group 2: $0.0109 \pm 0.0125, P=0.27$.

Mean \pm SD weeks from enrolment to healing: Group 1: $9.69 \pm 3.28$; Group 2: $7.98 \pm 4.41, P=0.41$.

Mean \pm SD total cost/completed patient (price year not stated, based on clinician time plus materials plus number of visits at USD 35/visit): Group 1: USD 901.73 \pm 576.45 ; Group 2: USD $559.41 \pm 290.75, P=$ 0.05 .

\section{Notes}

*This was the linear healing rate of the wound edge toward the wound centre. It is calculated as the change in wound area from baseline to endpoint divided by the average of baseline and endpoint wound perimeter measurements, after the method proposed by Gilman 1990.

Completed trial: Group 1: 11 patients; Group 2: 17 patients.

Numbers of patients (with reasons) who withdrew before completion: Group 1: 5 patients (1 allergy to Unna's Boot; 1 weeping dermatitis; 1 left town; 1 enrolled with exclusion criterion - immunosuppression; 1 had increasing ulcer size and was referred to surgeon). Group 2: 2 patients (1 enrolled with exclusion criterion - low ABPI; 1 not healing, referred to surgeon). 3 patients not accounted for in the paper.

Restricting selection of patients to those with relatively small ulcers is not likely to be representative of the target population seen in clinical practice.

Ulcers in Group 2: were of shorter baseline duration. No information about skill of care providers.

\section{Risk of bias}

\begin{tabular}{lll} 
Bias & Authors' judgement & Support for judgement \\
\hline $\begin{array}{l}\text { Random sequence genera- } \\
\text { tion (selection bias) }\end{array}$ & Unclear risk & $\begin{array}{l}\text { No details provided. "A multicenter, prospective, randomised, parallel-group } \\
\text { study was conducted...." }\end{array}$ \\
\hline
\end{tabular}


DePalma 1999 (Continued)

Allocation concealment Unclear risk No details provided. "A multicenter, prospective, randomised, parallel-group (selection bias)

Incomplete outcome data High risk (attrition bias) study was conducted....".

38 people randomised; 10 withdrew, but unclear whether included in analysis; 3 of the withdrawals unaccounted for (unclear from which group they came).

All outcomes

\begin{tabular}{|c|c|c|}
\hline $\begin{array}{l}\text { Blinded outcome assess- } \\
\text { ment (healing) }\end{array}$ & Unclear risk & $\begin{array}{l}\text { "At each ... a tracing of the ulcer outline was made on clear film ..." "Data } \\
\text { sheets and ulcer tracings were sent to the study coordinator for tabulation and } \\
\text { analysis...". }\end{array}$ \\
\hline
\end{tabular}

Baseline comparability High risk Ulcers in Group 2 were of shorter mean duration.

Duby 1993

Methods RCT (no details on methods). Setting: UK, no other details reported.

Participants 67 patients (76 legs) recruited (source population not described).

Inclusion criterion: $\mathrm{ABPI} \geq 0.9$. No other patient selection criteria stated.

Number of male:female patients (limbs) : Group 1: 4 (5 limbs):16 (20 limbs); Group 2: 7 (7 limbs):16 (18

limbs); Group 3: 11 (12 limbs):13 (14 limbs).

Mean (range) patient age in years: Group 1: 72.6 (47-89); Group 2: 70.1 (47-85); Group 3: 72.9 (56-86).

Mean (range) baseline ulcer area in $\mathrm{cm}^{2}$ : Group 1: 13.1 (1.1-29.4); Group 2: 11.9 (1.0-40.3); Group 3: 12.3

(1.5-30.1).

Mean baseline ulcer duration in months: Group 1: 26.7; Group 2: 20.5; Group 3: 34.5.

Interventions

Group 1: SSB system comprising: orthopaedic wool; 2 or more layers of SSB applied in counter-rotating directions (Comprilan); and net covering (Tricofix). Bandages washed and reused. $(n=20$ patients/25 limbs).

Group 2: 4LB system comprising: orthopaedic wool; crepe bandage; elastic bandage (Elset); and elastic cohesive bandage (Coban). New bandages applied at each dressing change. ( $n=23$ patients $/ 25$ limbs).

Group 3: paste-bandage system comprising: zinc and ichthammol paste bandage (Icthopaste); cotton crepe bandage (Elastocrepe); and elastic tubular bandage (Tubigrip) ( $n=24$ patients/26 legs).

All patients: ulcers irrigated with saline, then a non-adherent dressing was applied (Cuticerin). Bandages changed as required, according to exudate and slippage (mean rate twice weekly for all groups).

Outcomes $\quad$ Number (\%) limbs with complete healing at 12 weeks: Group 1: 10/25 (40\%); Group 2: 11/25 (44\%); Group 3: 6/26 (23\%). Authors reported that the differences for Group 1 versus Group 3, and Group 2 versus Group 3, were significant, but P values not shown.

Mean percentage reduction in baseline ulcer area at 12 weeks: Group 1: 60\%; Group 2: 76\%; Group 3: $43 \%$. Authors reported that the difference between Groups 1 and 2 was not significant, but P value not shown.

Notes

Higher proportion of males in Group 3: 11/24 compared to 11/43 in other 2 groups combined. Longer baseline ulcer duration in Group 3. Ulcer area determined weekly using tracings from photographs combined with computerised planimetry. Change in leg volume during the 12-week trial was reported. Data from limbs of same patient are likely to be highly correlated and could bias estimates of treatment effect.

\section{Risk of bias}

\section{Bias}

Authors' judgement Support for judgement 
Duby 1993 (Continued)

Random sequence genera- Unclear risk "The treatments were randomised to each patient in the following mantion (selection bias) ner..." (goes on to give only numbers receiving each treatment).

Allocation concealment Unclear risk
(selection bias)

No details provided. "The treatments were randomised to each patient in (selection bias) the following manner ..." (goes on to give only numbers receiving each treatment).

Incomplete outcome data Low risk (attrition bias) Complete healing reported on all 67 people randomised; less clear for continu-

All outcomes ous outcomes.

Blinded outcome assess- Unclear risk No details given.
ment (healing)

Baseline comparability High risk Baseline ulcer duration varied across 3 groups, longer in Group 3.

Eriksson 1984

\begin{tabular}{ll} 
Methods & RCT, open design, outpatient setting, Sweden. \\
\hline Participants & 53 patients recruited to Part I of the trial ( 13 male, 40 female; mean age 70.1 years). \\
& 44 patients recruited to Part II ( 9 patients excluded because of ulcer healing or reasons unrelated to the \\
trial). & Inclusion criteria: not stated. \\
& Exclusion criteria: overt diabetes mellitus, arterial insufficiency defined as ABPI < 0.75, erysipelas, cel- \\
lulitis.
\end{tabular}

Interventions

Part I (2 weeks' duration): patients randomised to receive either gauze moistened with normal saline or dextranomer beads (Debrisan). Numbers of patients/group not clear.

Part II (8 weeks' duration): patients were re-randomised to the following groups:

Group 1: ulcer cleansed with saline followed by application of freeze-dried porcine skin dressing (Skintec). Dressings changed every other day. No compression applied $(n=11)$. Patients crossed over to the bandage system received by Group 3 mid study, because the porcine skin dressing was no longer available.

Group 2: ulcer cleansed with saline, followed by application of non-adherent aluminium foil dressing (Metallina). Dressings changed every other day. No compression applied $(n=20)$.

Group 3: zinc oxide paste-impregnated inner stocking (ACO) plus outer elastic bandage (Tensoplast) applied after resting with legs elevated for 30 minutes. Changed every $1-2$ weeks $(n=13)$.

Outcomes Part I: no statistically significant differences between groups for changes in ulcer area and volume.

Part II: mean (presumed, not stated) decrease in ulcer area:volume at 8 weeks: Group 1: 65\%:75\%; Group 2: 10\%:0\%; Group 3: 80\%:90\% (NB: values recorded from figure; findings of tests of statistical significance for between-group differences not reported).

Notes

Ulcer area and volume measured using stereophotogrammetry every 2 weeks. Baseline ulcer area:volume and duration not stated. Withdrawals: Group 1: no information; Group 2: 6 patients had treatment interrupted because of increase of the ulcers and/or signs of clinical infection; Group 3: no patients discontinued treatment.

\section{Risk of bias}


Eriksson 1984 (Continued)

Random sequence genera- Unclear risk "The investigation was designed as a randomised open trial". No further detion (selection bias) tails provided.

Allocation concealment Unclear risk

"The investigation was designed as a randomised open trial". No further de(selection bias) tails provided.

Incomplete outcome data High risk

(attrition bias)

All outcomes
"The treatment with porcine skin had to be stopped in the middle of the study as the dressing was no longer available... Treatment with double layer bandage was then introduced ... ". 6 patients in Group 2 had treatment interrupted because of increase of the ulcers and/or signs of clinical infection; no patients in Group 3 had treatment discontinued - however, unclear whether these people were analysed. There was no information about withdrawals from Group 1.

Blinded outcome assess- Unclear risk Not stated.

ment (healing)

Baseline comparability Unclear risk No baseline data presented.

\section{Eriksson 1986}

\begin{tabular}{ll}
\hline Methods & RCT, open design. Outpatient setting in Sweden. \\
\hline Participants & $\begin{array}{l}34 \text { outpatients with chronic venous leg ulcers (9 males, mean age } 66.9 \text { years; } 25 \text { females, mean age } 74.3 \\
\text { years). } 3 \text { diabetic patients in Group } 2 . \\
\text { Inclusion and exclusion criteria not specified. }\end{array}$
\end{tabular}

Interventions All patients: ulcerated limb immersed for 15 minutes in a bath of tepid potassium permanganate solution, then crusts and debris removed.

Group 1: inner stocking impregnated with zinc oxide paste (ACO) plus an outer elastic bandage (Tensoplast or Porelast Acryl). Bandages changed every 1-2 weeks $(n=17)$.

Group 2: hydrocolloid dressing (Duoderm) plus elastic bandage (Wero). Dressing renewed 1-2 times/ week. Bandage removed at night and reapplied in the morning by patients $(n=17)$.

\section{Outcomes}

Number (\%) patients with complete healing at 12 weeks: Group 1: 7/17 (41\%); Group 2: 9/17 (53\%). Statistical tests not reported.

Mean decrease in ulcer area:volume at 12 weeks: Group 1: 75\%:75\%; Group 2: 70\%:55\% (NB: values recorded from figure; all between-group differences reported as not statistically significant, but $P$ values not shown).

Number (\%) patients who discontinued treatment (with reasons): Group 1: 3/17 (18\%) (1 withdrew, 2 had infection of peri-ulcer skin); Group 2: 2/17 (12\%) (1 withdrew, 1 had enlargement of study ulcer and development of new ulcer).

Notes Ulcer area and volume measured using stereophotogrammetry every 2 weeks.

\section{Risk of bias}

\begin{tabular}{lll}
\hline Bias & Authors' judgement & Support for judgement \\
\hline $\begin{array}{l}\text { Random sequence genera- } \\
\text { tion (selection bias) }\end{array}$ & Unclear risk & "The study was designed as a randomised open trial ....".
\end{tabular}


Eriksson 1986 (Continued)

$\begin{aligned} & \text { Allocation concealment } \\ & \text { (selection bias) }\end{aligned} \quad$ Unclear risk $\quad$ "The study was designed as a randomised open trial ....".

Incomplete outcome data Unclear risk (attrition bias)

All outcomes

Blinded outcome assess- Unclear risk Not stated.
ment (healing)

For healed outcome, only numerators given, therefore unclear whether all patients followed-up. Numbers for continuous outcomes unclear.

Baseline comparability Unclear risk No baseline data reported.

Franks 2004

$\begin{array}{ll}\text { Methods } & \text { RCT (multicentred) with stratification according to study centre and baseline ulcer area }\left(\leq 10 \mathrm{~cm}^{2} \text { or }>\right. \\ \left.10 \mathrm{~cm}^{2}\right) . \text { Patients randomised to } 1 \text { of } 2 \text { bandage systems and to } 1 \text { of } 2 \text { primary dressings, using a facto- } \\ \text { rial design. Sample size: target sample of } 240 \text { patients overall could not be recruited. The authors es- } \\ \text { timated that } 159 \text { patients overall provided } 81 \% \text { power to detect } 15 \% \text { difference in healing rates at } 5 \% \\ \text { significance level. }\end{array}$

Participants

159 patients recruited from 12 community leg ulcer clinics in the UK (156 patients were evaluated). Inclusion criteria: patient age $\geq 18$ years; venous leg ulceration with wound aetiology confirmed using clinical history and $\mathrm{ABPI} \geq 0.8$; minimum baseline ulcer duration 2 weeks; maximum baseline ulcer duration 52 weeks.

Exclusion criteria: pregnancy; causes of ulceration other than venous disease; active cellulitis treated with systemic antibiotics; dry, non-exuding wounds; previous entry to trial.

Number of male:female patients: Group 1: 27:47; Group 2: 34:48.

Mean \pm SD patient age in years: Group 1: $67.5 \pm 14.3$; Group 2: $70.9 \pm 13.4$.

Proportions of patients with baseline ulcer size $\leq 10 \mathrm{~cm}^{2}:>10 \mathrm{~cm}^{2}$ : Group 1: 80\%:20\%; Group 2: 82\%:18\%.

Baseline median (range) baseline ulcer area in $\mathrm{cm}^{2}$ : Group 1: 5.0 (0.3-115.8); Group 2: 3.5 (0.5-123.1).

Median (range) baseline ulcer duration in weeks: Group 1: 8 (2-40); Group 2: 8 (2-40).

Number (\%) patients with previous ulceration: Group 1: 29/74 (39\%); Group 2: 28/82 (34\%).

Number (\%) patients with DVT: Group 1: 14/74 (19\%); Group 2: 8/82 (10\%).

Number (\%) patients chair or bed bound; walking with aid; walking freely:

Group 1: 0/74 (0\%); 18/74 (24\%); 56/74 (76\%);

Group 2: $1 / 82$ (1\%); 14/82 (17\%); 67/82 (82\%).

Number (\%) patients with limb: fully mobile; limited; fixed:

Group 1: 54/74 (73\%); 17/74 (23\%); 2/74 (3\%);

Group 2: $73 / 82(89 \%) ; 9 / 82(11 \%) ; 0 / 82(0 \%)$.

All patients: study limb washed using emollient dissolved in tap water, wound debrided where necessary, and a hypoallergenic hydrating cream applied to the surrounding skin. In addition to the bandage comparison, patients were randomised to 1 of 2 foam dressings (Allevyn or Mepilex) prior to bandaging. Dressings and bandages were reapplied at least weekly.

Group 1: foam dressing as above (52.7\% patients received Allevyn) plus 4LB (Flexiban, Setocrepe, Elset, Coban) $(n=74)$.

Group 2: foam dressing as above (51.2\% patients received Allevyn) plus SSB (Flexiban, Actico) $(n=82)$.

Patients with ulcer closure before the end of the trial provided with class II compression stockings and followed-up until 24 weeks. Patients who withdrew from randomised treatment were allocated to an alternative treatment and followed-up until wound closure or 24 weeks. 
Franks 2004 (Continued)

Outcomes
Number (\%) patients with complete healing at 24 weeks (for those remaining on randomised treatment): Group 1: 51/74 (69\%); Group 2: 60/82 (73\%) (P value not reported).

Number (\%) patients with complete healing at 24 weeks (intention-to-treat analysis): Group 1: 59/74 (80\%); Group 2: 62/82 (76\%).

Kaplan-Meier analysis: cumulative healing rates at 12 weeks were $56 \%$ in both groups; and at 24 weeks Group 1: 85\%; Group 2: 83\%.

HR for healing adjusted for study centre, treatment and baseline ulcer area, by intention-to-treat was 1.08 in favour of Group $2(95 \% \mathrm{Cl} 0.63-1.85), \mathrm{P}=0.79$.

HR for healing for subgroup of patients requiring aid with walking (Group 1: $n=18$; Group 2: $n=14$ ), by intention-to-treat was 1.35 in favour of Group 2 (95\% $\mathrm{Cl} 0.60-3.03), \mathrm{P}=0.46$.

Quality of life assessment: patients completed Nottingham Health Profile at baseline, at healing or withdrawal and at 24 weeks (scores $0-100$, with lower scores indicating better quality of life). Domains include: energy; bodily pain; emotional reactions; sleep; social isolation; and physical mobility. Mean differences in final scores calculated using linear regression with adjustment for baseline scores. 139/156 (89\%) patients completed at least 1 follow-up questionnaire (66 in Group 1, 73 in Group 2). Overall, statistically significant improvements were observed for all scores at 24 weeks. Improvement was greater for patients with healed limbs $(n=114)$ compared to those who remained unhealed $(n=$ 40), the mean difference for the following domains were statistically significant: bodily pain (MD 13.2, $95 \% \mathrm{Cl} 3.6-22.9, \mathrm{P}=0.008$ ), emotional reactions ( $\mathrm{MD} 10.5,95 \% \mathrm{Cl} 2.8-18.1, \mathrm{P}=0.007$ ) and social isolation (MD 8.5, 95\% Cl 1.2-15.9, $\mathrm{P}=0.024$ ); a clinically significant difference was seen for sleep (MD 11.2, 95\% $\mathrm{Cl} 0.0-22.5, \mathrm{P}=0.051)$. No statistically significant differences observed between scores for any domain from the 2 treatment groups.
In patients with bilateral ulceration, the limb with the largest total ulcerated area was studied.

$3 / 159$ patients excluded from the analysis ( 2 ineligible; 1 withdrew after 1 week).

Of 156 remaining patients, number (\%) withdrawals during trial: Group 1: 16/74 (22\%); Group 2: 17/82 $(21 \%)$.

Reasons for withdrawal: Group 1: infection 3; peri-ulcer skin maceration 2; other bandage-related reason 2; patient request 2; lost to follow-up 6; dressing-related 1. Group 2: infection 1; peri-ulcer skin maceration 2; other bandage-related reason 3; patient request 2; lost to follow-up 9.

Adverse events: Group 1: 23 patients experienced 30 adverse events; Group 2: 22 patients experienced 36 adverse events.

Number of adverse events related to bandage (none; possible; definite): Group 1: 18; 6; 6; Group 2: 27; $2 ; 7$.

Number of different types of adverse events possibly or definitely device-related: Group 1: tissue damage or new ulcer 2; eczema or reaction to bandage 2; pain 2; maceration 2; other 4; Group 2: tissue damage or new ulcer 3; eczema or reaction to bandage 2; pain 2; maceration 2.

All bandages applied according to the manufacturers' instructions.

Ulcers measured using transparency tracing combined with computerised planimetry.

\section{Risk of bias}

\begin{tabular}{lll}
\hline Bias & Authors' judgement & Support for judgement \\
\hline $\begin{array}{l}\text { Random sequence genera- } \\
\text { tion (selection bias) }\end{array}$ & Low risk & $\begin{array}{l}\text { Information from published trial report was unclear: } \\
\text { "Patients were randomised to a bandage system ...", however, standard da- } \\
\text { ta checks undertaken for the IPD meta-analysis suggested that the random se- } \\
\text { quence generation was satisfactory. }\end{array}$ \\
\hline
\end{tabular}


Franks 2004 (Continued)
Allocation concealment
Low risk
"Randomization took place ... by means of opening sealed envelopes in se- (selection bias) quential order". The trial investigators told us that these envelopes were num- bered.

\begin{tabular}{|c|c|c|}
\hline $\begin{array}{l}\text { Incomplete outcome data } \\
\text { (attrition bias) }\end{array}$ & Low risk & $\begin{array}{l}\text { "The analysis based on ITT meant that patients remained in their original ran- } \\
\text { domised groups irrespective of subsequent treatments applied". }\end{array}$ \\
\hline 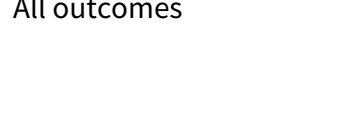 & & $\begin{array}{l}3 \text { randomised patients were excluded from the analysis "... two due to signifi- } \\
\text { cant arterial disease... and one who had not given informed consent and who } \\
\text { withdrew at } 1 \text { week". }\end{array}$ \\
\hline
\end{tabular}

All 3 patients were reinstated for the IPD meta-analysis.

\begin{tabular}{lll}
\hline $\begin{array}{l}\text { Blinded outcome assess- } \\
\text { ment (healing) }\end{array}$ & High risk & Trial authors confirmed that assessment of healing was not blind to treatment. \\
\hline Baseline comparability & Low risk & $\begin{array}{l}\text { Groups appeared balanced at baseline; randomisation was stratified for ulcer } \\
\text { area. }\end{array}$ \\
\hline
\end{tabular}

Gould 1998

$\begin{array}{ll}\text { Methods } & \text { RCT in outpatient leg ulcer clinic in Truro, UK. Blinded outcome assessment (3 separate treatment } \\ \text { rooms used for removal of bandages, clinical evaluation of ulcer, and reapplication of bandage). }\end{array}$
Participants 39 patients with 46 ulcers $(7$ had bilateral ulcers) recruited from local GPs. Inclusion criteria: venous ulcers, $\mathrm{ABPI}>0.8$, ambulatory.
Exclusion criteria: arterial or mixed ulcers, diabetes mellitus, peripheral neuropathy, congestive heart failure, chronic renal or liver disease, infected wounds, ankle circumference $<18 \mathrm{~cm}$ or $>25 \mathrm{~cm}$, known sensitivity to paste bandages, ulcer duration $<2$ months.
Mean (range) patient age: 71.5 years $(44-87)$.
Mean (range) baseline ulcer area: $7.44 \mathrm{~cm}^{2}$ (0.2-60.2).
Mean baseline duration of ulcers: 10 months.
Trial authors reported no statistically significant differences between groups in relation to baseline variables, but data were not presented per group.

Interventions

Group 1: 3-component compression system comprising: medicated paste bandage, elastic bandage (Setopress), and elasticated viscose stockinette ( $\mathrm{n}=19$ patients).

Group 2: 3-component compression system comprising: medicated paste bandage, cotton crepe bandage (Elastocrepe), and elasticated viscose stockinette ( $n=20$ patients).

All patients: elastic bandage (Setopress) for 1 week prior to start of randomised treatment; potassium permanganate soaks for 5 minutes prior to application of compression; provided with class II compression stockings post-healing and followed up by GP.

\section{Outcomes}

Analyses based on 32 patients with 39 ulcers.

Number (\%) patients with complete healing at 15 weeks: Group 1: 11/19 (58\%); Group 2: 7/20 (35\%), P= 0.24 .

Withdrawals: 7/39 (18\%) patients withdrew overall (full breakdown/group not reported). 4 withdrew following initial assessment, 1 after 3 weeks because of ulcer deterioration (Group 2), and 2 excluded because ineligible (ulcers $<2$ months duration at baseline).

\section{Notes}

When there were several ulcers on one leg, the largest wound was included in the trial. In the case of bilateral ulceration, each leg was considered separately, and the largest ulcer on each leg was studied; long-stretch bandage applied to 1 leg at random and SSB to the other leg. 
Gould 1998 (Continued)

Some healing data highly correlated because of those patients with two ulcerated limbs; no adjustment made for this in the statistical analysis.

Ulcer areas measured using transparency tracing and computerised planimetry. Ulcers photographed every 2 weeks.

\section{Risk of bias}

\begin{tabular}{lll}
\hline Bias & Authors' judgement & Support for judgement \\
\hline $\begin{array}{l}\text { Random sequence genera- } \\
\text { tion (selection bias) }\end{array}$ & Unclear risk & $\begin{array}{l}\text { No details provided. "The trial was a prospective, randomised, observer-blind, } \\
\text { parallel group study... ". }\end{array}$ \\
\hline $\begin{array}{l}\text { Allocation concealment } \\
\text { (selection bias) }\end{array}$ & Unclear risk & $\begin{array}{l}\text { No details provided. "The trial was a prospective, randomised, observer-blind, } \\
\text { parallel group study ... ". }\end{array}$ \\
\hline $\begin{array}{l}\text { Incomplete outcome data } \\
\text { (attrition bias) } \\
\text { All outcomes }\end{array}$ & High risk & $\begin{array}{l}\text { 39 patients randomised "... 32 patients were available for analysis". With- } \\
\text { drawals not reported by group. }\end{array}$ \\
\hline $\begin{array}{l}\text { Blinded outcome assess- } \\
\text { ment (healing) }\end{array}$ & Low risk & $\begin{array}{l}\text { "Assessments were undertaken weekly at the clinic ... Three separate rooms } \\
\text { were used respectively for the removal of the bandages, for the clinical evalua- } \\
\text { tion and for the application of new bandages. This ensured that clinical evalu- } \\
\text { ation was carried out blind to the bandage system used". }\end{array}$ \\
\hline
\end{tabular}

Baseline comparability Unclear risk Described as comparable, but data by group not presented.

Harley 2004

Methods RCT, no further details of methods. Community setting in Tasmania, Australia. No sample size estimation presented. Initially sought 40 patients, but only 30 recruited.

\section{Participants}

30 patients attending a hospital leg ulcer clinic for the first time for treatment of chronic venous ulceration.

Inclusion criteria: venous ulceration confirmed by clinical assessment, Doppler ultrasound and ABPI at least 0.8 ; not previously treated with compression; ulcer of at least 1 months' duration and $2 \mathrm{~cm}$ or more at widest point.

Exclusion criteria: not stated.

Number of male:female patients: Group 1: 8:6; Group 2: 5:11.

Average - unclear whether this was mean or median - (range) patient age in years: Group 1: 75 (46-91); Group 2: 71 (38-95).

Trial authors stated that average number of co-morbidities/patient did not differ significantly between treatment groups, but no further details provided.

No information on baseline ulcer area or duration. 
Harley 2004 (Continued)

All patients: treatment provided by community nursing services; patients attended leg ulcer clinic every 6 weeks for assessment. Ulcers that healed between clinic visits examined by a specialist nurse from the leg ulcer clinic. All patients followed-up until healing.

Outcomes

Number (\%) patients with complete healing during trial - follow-up point unclear (3 months?): Group 1: 8/14 (57\%); Group 2: 13/16 (81\%) ( $P=0.151$, chi-squared analysis).

Average days spent on treatment (unclear whether mean or median, and unclear whether this related to time to healing): Group 1: 63; Group 2: 87 (difference described as not significant at 5\% level but $P$ value and Cls not shown; chi-squared analysis of quintiles of healing times $\mathrm{P}=0.702$ ).

Number (\%) patients reporting at least one adverse event: Group 1: 14/14 (100\%); Group 2: 10/16 (63\%).

Chi-squared analysis of 3 categories of number of adverse events $(0 ; 1 ; 2$ or more) $P=0.013$ in favour of Group 2.

Average number of adverse events/patient (unclear whether mean or median): Group 1: 1.64; Group 2: 0.75 (difference described as significant at $1 \%$ level but $\mathrm{P}$-value and $\mathrm{Cls}$ not reported).

Types of adverse events in order of prevalence (numbers not reported): Group 1: slipping, pain, pressure to length of shin, wound infection; Group 2: slipping, excess firmness reported by patient, itching, venous eczema.

Average number of incidents of inappropriate pressure defined as pain, redness or horizontal wrinkles on limb (unclear whether mean or median): Group 1: 5.43; Group 2: 2.31 (difference described as significant at $5 \%$ level but $P$ value and $\mathrm{Cl}$ n not shown; chi-squared analysis of quintiles $\mathrm{P}=0.03$ in favour of Group 2).

Number (\%) patients who withdrew (reasons not reported): Group 1: 6/14 (43\%); Group 2: 1/16 (6\%) (P $=0.018$, chi-squared analysis).

Total cost of 6 weeks' treatment based on bandage costs excluding primary dressings (AUD, price year appears to be 1999-2000): Group 1: AUD 35.00; Group 2: AUD 114.00. plained further).

Held a series of workshops with community nurses to educate them with regard to the trial and bandaging systems prior to commencement of data collection; further support provided during trial from the leg ulcer clinic. Community nurses were already experienced in applying the $4 \mathrm{LB}$ at the start of the trial.

Additional outcomes assessed in the trial were:

Ease of application of bandages: assessed by nurses completing a questionnaire at each dressing change, indicating that nurses were confident in applying both compression systems.

Patient comfort: categorised as completely comfortable vs not completely comfortable: Group 1: 2 vs 12; Group 2: 12 vs $4(P=0.001)$.

Non-routine bandage changes required/group significantly higher for Group 1 in terms of both number of patients requiring this and average number of non-routine changes/patient.

\section{Risk of bias}

\section{Bias \\ Authors' judgement Support for judgement}

Random sequence genera- Unclear risk tion (selection bias)
"Those consenting were randomly allocated to one of the two groups".

"The patient was then randomly allocated to a treatment method". 
Harley 2004 (Continued)

Allocation concealment $\quad$ Unclear risk No information provided.
(selection bias)

\begin{tabular}{ll}
\hline $\begin{array}{l}\text { Incomplete outcome data } \\
\text { (attrition bias) }\end{array}$ & Low risk \\
All outcomes & $\begin{array}{l}\text { "The study ... followed all patients ... through to healing of the ulcer, cessa- } \\
\text { tion of compression bandaging and transfer of the patient into compression } \\
\text { stockings". }\end{array}$
\end{tabular}

\begin{tabular}{lll}
\hline $\begin{array}{l}\text { Blinded outcome assess- } \\
\text { ment (healing) }\end{array}$ & Unclear risk & No information provided. \\
\hline Baseline comparability & Unclear risk & No information provided about baseline wound area and duration. \\
\hline
\end{tabular}

Hendricks 1985

Methods $\quad$ RCT (no details about allocation methods). Setting: outpatients, USA.

\section{Participants}

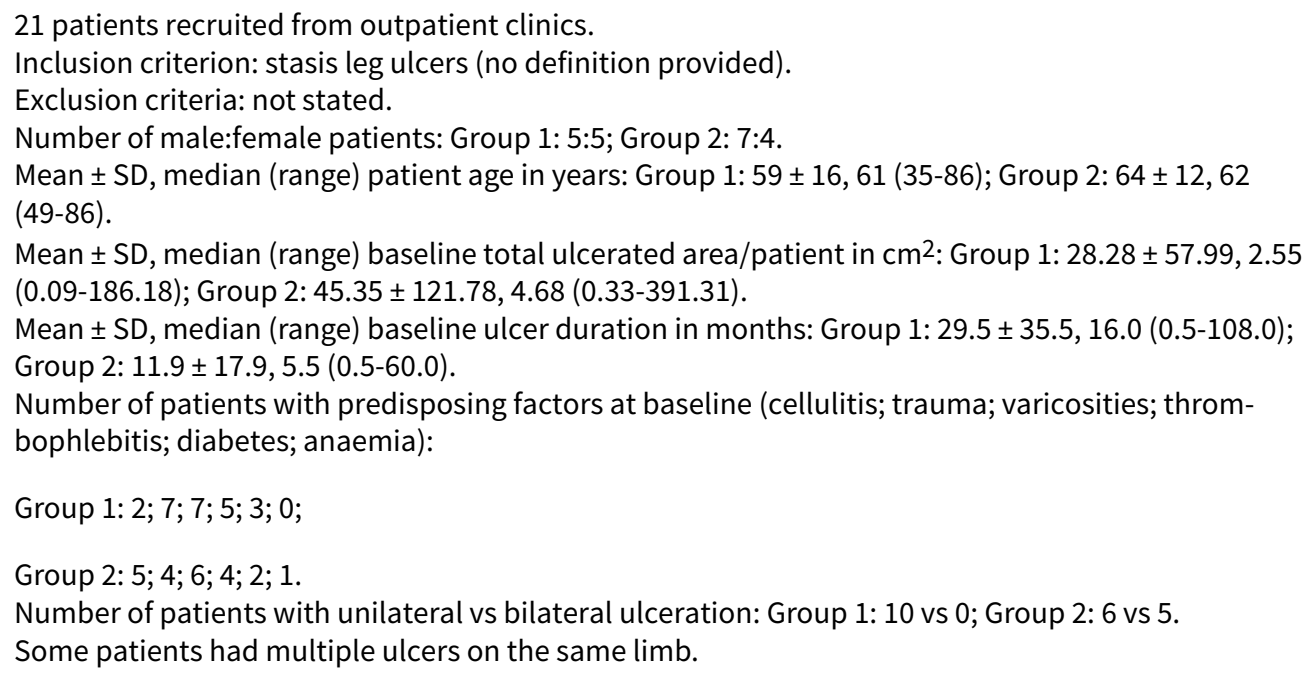

Group 1: Unna's Boot compression system consisting of: zinc oxide and calamine paste-impregnated bandage (Dome-Paste); gauze bandage (Kerlix); and elastic bandage. Prior to bandage application, sharp debridement of ulcer was undertaken followed by wound cleansing with $3 \% \mathrm{H}_{2} \mathrm{O}_{2}$ and bacitracin/polymyxin ointment (Polysporin) application to ulcer surface. For exuding ulcers, the wound was dried using a hair-dryer and $1 \%$ gentian violet applied. A low-potency corticosteroid cream (Tridesilon $0.05 \%$ ) was applied to the peri-wound skin. The ulcer was covered with gauze, and sometimes foam dressing. Dressings and bandages were changed during clinic visits every 3-9 days, depending on exudate ( $\mathrm{n}=10$ patients).

Group 2: open-toe, below-knee, elastic compression stocking ( $24 \mathrm{mmHg}$ at ankle graduating to 16 $\mathrm{mmHg}$ at calf) (Futuro) applied by patients each morning and removed at bedtime. Patients were instructed to dry ulcers following bath or shower using gauze dressing, prior to cleansing ulcers twice daily using $3 \% \mathrm{H}_{2} \mathrm{O}_{2}$. Then Polysporin ointment was applied to the ulcer surface, and Tridesilon $0.05 \%$ to peri-wound skin. A gauze dressing, retained with cloth tape (Dermicel), was applied, and sometimes a foam dressing was used. Patients attended clinic every 1 or 2 weeks, when sharp debridement was carried out ( $n=11$ patients).

All patients: concurrent treatments included: systemic antibiotics as deemed appropriate following ulcer cultures; oral zinc sulphate in cases of zinc deficiency; diuretics as necessary; reducing diet if overweight. 
Hendricks 1985 (Continued)

If patients were not deemed to be making progress at the end of each month - in terms of decreasing ulcer size and also other outcomes relating to changing limb volume - they were re-assigned to the alternate study group.

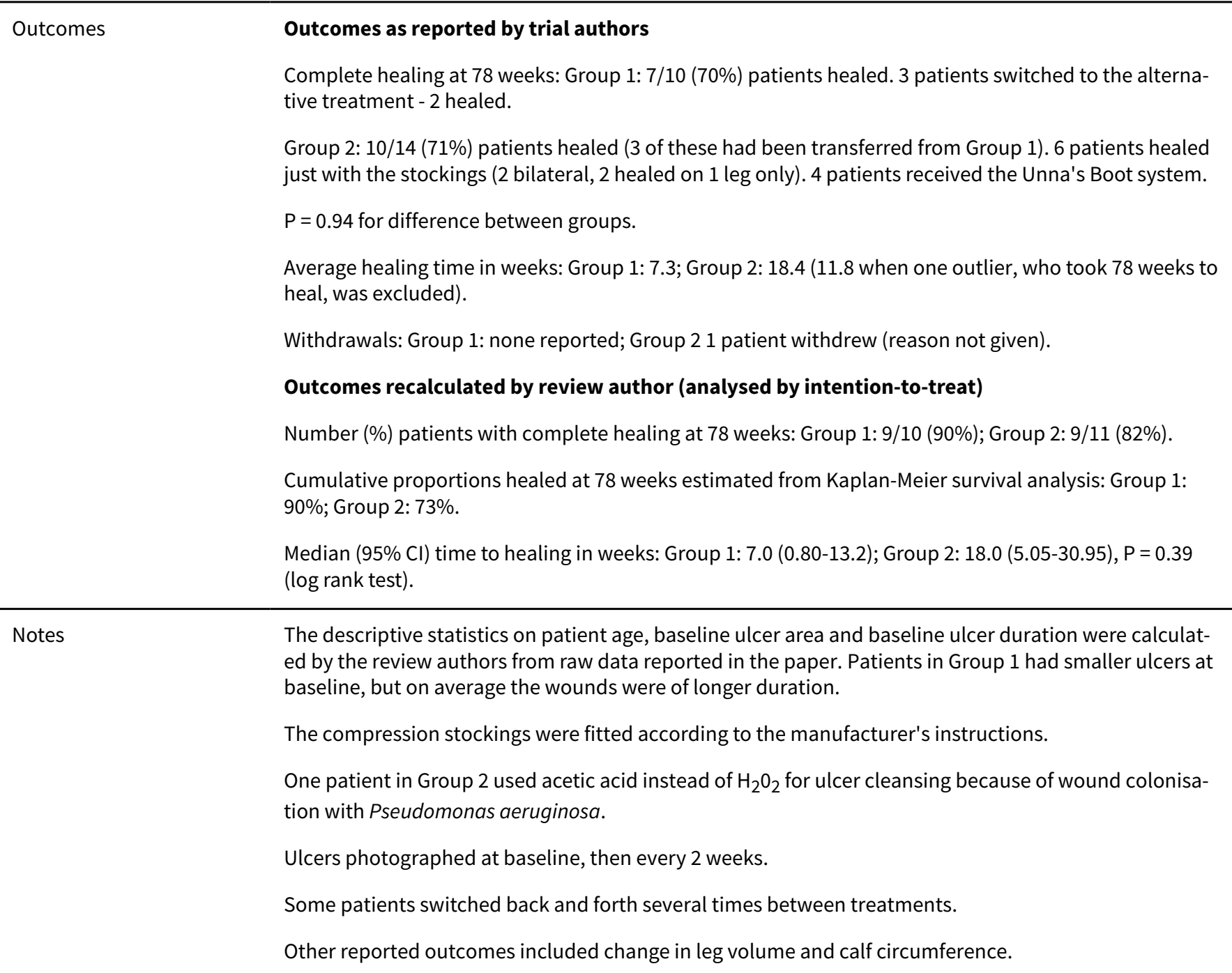

\section{Risk of bias}

\begin{tabular}{lll}
\hline Bias & Authors' judgement & Support for judgement \\
\hline $\begin{array}{l}\text { Random sequence genera- } \\
\text { tion (selection bias) }\end{array}$ & Unclear risk & "The 21 patients were randomly assigned to two groups ... ". \\
\hline $\begin{array}{l}\text { Allocation concealment } \\
\text { (selection bias) }\end{array}$ & Unclear risk & "The 21 patients were randomly assigned to two groups ... ". \\
\hline $\begin{array}{l}\text { Incomplete outcome data } \\
\text { (attrition bias) } \\
\text { All outcomes }\end{array}$ & Low risk & $\begin{array}{l}21 \text { participants were randomised and endpoint data is presented for 20 partici- } \\
\text { pants (1 withdrawal from Group 2). }\end{array}$ \\
\hline $\begin{array}{l}\text { Blinded outcome assess- } \\
\text { ment (healing) }\end{array}$ & Unclear risk & "Pictures of the ulcers were taken initially and every 2 weeks". \\
\hline
\end{tabular}


Hendricks 1985 (Continued)

Baseline comparability High risk Imbalances for baseline ulcer area (larger in Group 2) and duration (older in Group 1).

RCT (multicentred, pragmatic, i.e. reflecting everyday clinical practice as far as possible). Randomisation stratified by study centre, previous ulceration (yes/no), ulcer area ( $\leq$ or $>10 \mathrm{~cm}^{2}$ ) and ulcer duration ( $\leq$ or $>6$ months). Randomisation code developed using computer-generated permuted blocks (randomly sized 4 or 6). Patients and nurses aware of allocated treatment after assignment. Sample size estimation: 200 patients/arm would provide $80 \%$ power to detect $15 \%$ difference in healing rates at 12 weeks at $5 \%$ significance level. Patients were followed-up for a minimum of 12 months.

Participants

387 patients recruited from 9 community (leg ulcer services, district nursing or general practice) and outpatient (vascular surgery) centres in the UK.

Inclusion criteria: patients with venous leg ulcer $\geq 1 \mathrm{~cm}$ diameter.

Exclusion criteria: age < 18 years; $\mathrm{ABPI}<0.8$; diabetes mellitus; previous unsuccessful use of a trial bandage.

Number of male:female patients: Group 1: 79:116; Group 2: 80:112.

Mean \pm SD (range) age in years: Group 1: $71.9 \pm 12.3$ (25-97); Group 2: $71.3 \pm 14.1$ (23-96).

Number (\%) patients fully mobile; needing assistance; immobile: Group 1: 123 (63\%); 72 (37\%); 0 (0\%); Group 2: 115 (60\%); 70 (37\%); 3 (2\%).

Number (\%) patients with full ankle mobility vs impairment vs fixed: Group 1: $131(67 \%)$ vs $59(30 \%)$ vs 3 (2\%); Group 2: 128 (67\%) vs $58(30 \%)$ vs $2(1 \%)$.

Median (range) number of ulcer episodes since first ulcer: Group 1: 2 (0-50); Group 2: 2 (0-64).

Mean \pm SD (range) ankle circumference in cm: Group 1: $23.9 \pm 2.9$ (16.2-34.0); Group 2: $23.9 \pm 2.9$

(16.0-32.3).

Median (range) ulcer duration in months: Group 1: 3 (0.5-456); Group 2: 3 (0.5-768).

Median (range) ulcer area in cm²: Group 1: 3.81 (0.19-254.58); Group 2: 3.82 (0.35-143.93).

Interventions

All patients: ulcers cleansed using tap water or saline and covered with simple low-adherent dressing. Dressings and bandages renewed by the usual nursing staff at least weekly.

Group 1: 4LB: orthopaedic wool padding, crepe retention bandage, class 3A compression bandage and cohesive compression bandage, all applied with $50 \%$ overlap. The original 4LB system and 2 proprietary kits (Profore and System 4) were randomly allocated $(n=195)$.

Group 2: SSB: orthopaedic wool padding covered with 1 or $2100 \%$ cotton short-stretch compression bandages (Comprilan or Rosidal K), applied using spiral, figure-of-8 or modified Putter techniques ( $\mathrm{n}=$ 192).

Outcomes

Number (\%) patients with complete healing at 4 months: Group 1: 107/195 (55\%); Group 2: 86/192 (45\%) (P value not reported - these data were shown as part of the discussion section for comparison with other trials).

Number (\%) patients with complete healing at 1 year: Group 1: 152/195 (78\%); Group 2: 138/192 (72\%) ( $P$ value not reported - these data were shown as part of the discussion section for comparison with other trials).

Kaplan-Meier estimate of median (95\% Cl) time to healing in days: Group 1: 92 (71-113); Group 2: 126 (95-157), log rank comparison $\mathrm{P}=0.117$.

Cox regression model used to assess impact of treatment centre, ulcer area, ulcer duration, ulcer episode, age weight, mobility, ankle mobility and ABPI on time to healing. Following adjustment for treatment centre, number of previous episodes, weight, baseline ulcer area, ulcer duration and ankle mobility, there was a statistically significant increase in the probability of healing in Group 1: HR 0.72 (95\% Cl 0.57-0.91). 
Kaplan-Meier estimate of cumulative proportion of legs healed at 12 weeks: Group 1: 46.3\%; Group 2: $36.7 \%$. Difference $9.6 \%(95 \% \mathrm{Cl} 0-20), P=0.1$.

Kaplan-Meier estimate of cumulative proportion of legs healed at 24 weeks: Group 1: 67.5\%; Group 2: 55.4\%. Difference $12.1 \%(95 \% \mathrm{Cl} 2-22), \mathrm{P}=0.02$.

Number (\%) withdrawals: Group 1: 46/195 (24\%); Group 2: 66/192 (34\%).

Number (\%) patients with non-bandage related adverse events: Group 1: 33/195 (17\%); Group 2: 39/192 (20\%).

Number (\%) patients with adverse events possibly related to compression treatment: Group 1: 76/195 (39\%); Group 2: 91/192 (47\%).

Cost-effectiveness and cost-utility analyses: perspective was UK NHS and Personal Social Service; time horizon was 1 year after recruitment; price year 2001; health benefit measured as differences in ulcer-free days (Kaplan-Meier estimate) and quality-adjusted life years (QALYs) estimated from patients' responses to the EuroQol-5D questionnaire. To account for censoring, QALYs were adjusted by the Kaplan-Meier survival estimate over the 1-year time horizon. Mean difference in healing time for ulcers was 10.9 days ( $95 \% \mathrm{Cl}-6.8-29.1$ ) in favour of Group 1: MD between treatment groups in QALYs was -0.02 (95\% Cl -0.08-0.04). The MD in total cost between compression systems was GBP 227.32/patient/year (95\% Cl 16.53-448.30) in favour of Group 1. Sensitivity analyses showed cost-effectiveness estimate to be robust to variation in number of bandages used and unit costs of compression systems. The 4LB emerged as the dominant strategy.

Notes

When patients had multiple ulcers, the limb with the largest eligible ulcer was studied. Healing defined as complete epithelial cover in the absence of a scab. At healing, the ulcer was photographed and healing was confirmed at the trial office by an investigator blind to treatment allocation. Training in the application of both types of bandages was provided during trial set-up.

This trial included an assessment of health-related quality of life. Since there was a large amount of missing data for this outcome, a descriptive analysis of findings was reported. The instruments used for data collection were the SF-12 and the Hyland Leg and Foot Ulcer Questionnaire. For the SF-12, scores between treatment groups appeared similar at baseline and over time for physical and mental components. For the Hyland Leg and Foot Ulcer Questionnaire, the scale was scored using 2 factors: practical, and emotional. Baseline and follow-up scores were similar between groups for both factors.

\section{Risk of bias}

\begin{tabular}{lll}
\hline Bias & Authors' judgement & Support for judgement \\
\hline $\begin{array}{ll}\text { Random sequence genera- } \\
\text { tion (selection bias) }\end{array}$ & Low risk & $\begin{array}{l}\text { "The randomisation code was developed using computer generated permut- } \\
\text { ed blocks, which were randomly of size four or six ... The allocation sequence } \\
\text { was generated by the trial statistician ... ". }\end{array}$ \\
& &
\end{tabular}

\begin{tabular}{|c|c|c|}
\hline $\begin{array}{l}\text { Allocation concealment } \\
\text { (selection bias) }\end{array}$ & Low risk & $\begin{array}{l}\text { "After the baseline clinical assessment ... the nurse recruiting the patient tele- } \\
\text { phoned the randomisation service ... .". }\end{array}$ \\
\hline
\end{tabular}

Incomplete outcome data Low risk (attrition bias) "Withdrawals from the trial and from allocated treatment were included in the All outcomes analysis by intention-to-treat (ITT)".

Blinded outcome assess- Low risk ment (healing)

\begin{abstract}
"Neither the patients nor the nurses administering the bandages and giving the associated care could be blinded... The nurse providing the regular leg ulcer care was responsible for documenting the assessments of ulcer progress every 4 weeks, including tracing the ulcer outline. These outcome assessors were therefore not blinded. The ulcer tracing was sent to the Trial Coordination Office where the ulcer area was determined by computerised planimetry by a researcher masked to bandage allocation".
\end{abstract}



ulcer took a Polaroid photograph of the healed ulcer and sent this to the Trial Coordination Office. An investigator unaware of the bandage allocation confirmed ulcer healing. This partially masked outcome assessment as the clinician only took a photograph when he/she had already decided the ulcer was healed".

Baseline comparability Low risk

Randomisation was stratified by ulcer area, ulcer duration, ulcer episode and clinical centre and resulted in good balance across groups. The primary analysis was also adjusted for important prognostic factors.

\section{Jünger 2004a}

Methods RCT (multicentred) with allocation by remote telephone service using a previously prepared centre-stratified randomisation list.

Aim of trial was to assess non-inferiority between 2 compression systems. Sample size: authors stated that non-inferiority was evaluated by comparing the $90 \% \mathrm{Cl}$ for between-group difference in complete healing with the non-inferiority limit of $15 \%$, assuming $65 \%$ healing rate, $80 \%$ power and $5 \%$ significance level. The required number of patients was not stated.

\section{Participants}

178 patients recruited from 4 study centres in France, Germany, Austria and Switzerland. Inclusion criteria: ambulatory $\geq 1 \mathrm{~h}$ /day; patient age $18-80$ years; venous leg ulceration confirmed using Doppler ultrasound; ulcer $<3$ months' baseline duration and maximum diameter $\leq 5 \mathrm{~cm} ; \mathrm{ABPI}>0.9$. Exclusion criteria: ulcers of diabetic, arterial or mixed aetiology; infected ulcers; co-morbidities (decompensated heart failure, cancer, chronic or autoimmune infection, insulin-dependent diabetes, diabetic neuropathy); restricted ankle movement.

Number of male:female patients: Group 1: 37:51; Group 2: 35:55.

Mean \pm SD (range) patient age in years: Group 1: $64.9 \pm 12.6$ (33-82); Group 2: $65.1 \pm 11.7$ (24-80). Number (\%) patients with recurrent ulceration: Group 1: 68/88 (77\%); Group 2: $69 / 90$ (77\%). Number (\%) patients with history of DVT: Group 1: 30/88 (34\%); Group 2: 29/90 (32\%). Mean \pm SD (range) baseline ulcer surface area in $\mathrm{mm}^{2}$ : Group 1: $240.3 \pm 229.7$ (27-1356); Group 2: $239.6 \pm$ 230.1 (23-1042).

Mean \pm SD (range) baseline ulcer duration in weeks: Group 1: $5.8 \pm 3.5$ (1-12); Group 2: $6.0 \pm 3.3$ (1-12).

Interventions

The following were disallowed for all participants during the trial: antibiotics, immunosuppressants, cytotoxic agents and venoactive drugs; new prescriptions or changes in dosage of all types of anti-inflammatory drugs; sclerotherapy, venous surgery and skin grafts. Patients were seen weekly and were asked to wear the compression device continuously between clinic visits. All patients had manual debridement, ulcer cleansing with normal saline and a non-medicated, non-adherent gauze primary dressing.

Group 1: tubular compression device; device was knitted, knee length, heel-less, open-toed, exerted graduated pressure, highest at ankle $(30-40 \mathrm{mmHg})$, corresponding to class III compression stockings $(n=88)$.

Group 2: SSB (Rosidal K) ( $\mathrm{n}=90)$.

Outcomes

Number (\%) patients with complete healing: Group 1: 51/88 (58.0\%); Group 2: $51 / 90$ (56.7\%). Between-group difference in proportion with complete healing $-1.3 \%$ (90\% Cl-13.5\%-10.9\%).

Mean \pm SD, median (range) time to healing in days: Group 1: $(n=51) 43.0 \pm 18.3$, 42 (13-84); Group 2: ( $n=$ 51) $43.6 \pm 18.3,42$ (13-85). Between-group difference for median $P=0.80$.

The Kaplan-Meier estimate showed no between-group difference in probability of healing $(P=0.41)$.

Number (\%) unhealed patients with reduction in ulcer area: Group 1: 25/37 (67.6\%); Group 2: 23/39 (59.0\%). 
Cox regression indicated that baseline ulcer area had a significant effect on time to healing $(P=0.002)$, but baseline ulcer duration and patient age were not significant predictors $(P=0.35$ and $P=0.82$ respectively).

Compliance with bandaging regimen (calculated as number of days compression device worn as a percentage of the number of days' participation in the study): Group 1: $96.8 \%$; Group 2: $96.4 \%(P=0.42)$.

Tolerability: Group 1: 12/88 (14\%) patients complained of pain in lower limb or sensation of tightness on the day after first application of compression, or 1-2 weeks later. This was resolved in all cases by using larger-sized devices. Group 2: no such problems.

Health-related quality of life assessed using the Nottingham Health Profile showed no difference between treatment groups (information taken from conference abstract,so only brief details available).

Notes

188 patients randomised, this comprised the intention-to-treat population, but data were presented on a total per protocol sample of 178. Reasons for exclusion: patient did not consent to use bandages 1 ; lost to follow-up 1; compression treatment used for $<1$ week 7 ; diabetes 1 (breakdown/group not reported).

Authors reported that results for the intention-to-treat population were comparable with those for the per protocol population, but did not report statistics.

Compression applied by investigator (described as 'experienced') or medical staff ('experienced and well trained') according to manufacturers' instructions. In discussion section, authors reiterated that all investigators were specialists, reducing problems with bandage application such as insufficient pressure or non-graduated pressure. Patients and family members were asked not to change the compression device.

Wounds measured weekly using transparency tracing combined with computerised planimetry.

\section{Risk of bias}

\begin{tabular}{lll}
\hline Bias & Authors' judgement & Support for judgement \\
\hline $\begin{array}{l}\text { Random sequence genera- } \\
\text { tion (selection bias) }\end{array}$ & Low risk & $\begin{array}{l}\text { "Once a patient was eligible, the investigator received the corresponding } \\
\text { treatment number (by telephone from an external randomisation centre) in } \\
\text { accordance with a previously prepared centre-stratified randomisation list". }\end{array}$ \\
\hline $\begin{array}{l}\text { Allocation concealment } \\
\text { (selection bias) }\end{array}$ & Low risk & $\begin{array}{l}\text { "Once a patient was eligible, the investigator received the corresponding } \\
\text { treatment number (by telephone from an external randomisation centre) in } \\
\text { accordance with a previously prepared centre-stratified randomisation list". }\end{array}$ \\
\hline $\begin{array}{l}\text { Incomplete outcome data } \\
\text { (attrition bias) } \\
\text { All outcomes }\end{array}$ & High risk & $\begin{array}{l}\text { Not an ITT analysis. 188 participants were randomised and as this was deemed } \\
\text { by the trialists a "non inferiority trial" they undertook a per protocol analysis } \\
\text { on only 178 participants. }\end{array}$ \\
\hline $\begin{array}{l}\text { Blinded outcome assess- } \\
\text { ment (healing) }\end{array}$ & Unclear risk & $\begin{array}{l}\text { "Change in ulcer size was evaluated by physicians drawing an outline of the } \\
\text { study ulcer on tracing paper. These tracings were then used to calculate the } \\
\text { area and diameter of the ulcers". }\end{array}$ \\
\hline $\begin{array}{l}\text { Baseline comparability } \\
\text { and risk }\end{array}$ & Low roups appeared comparable at baseline. \\
\hline
\end{tabular}

Jünger 2004b

Methods $\quad$ RCT (multicentred) with allocation achieved using blocks of 4 patients compiled by a contract research organisation prior to patient recruitment. Non-inferiority trial (non-inferiority margin set at $15 \%$ of healing rate). 
No a priori power calculation presented, but planned an interim analysis of first 120 patients to complete therapy to estimate final sample size or to terminate the study prematurely. Since the between-group difference in frequency of complete healing exceeded $15 \%$, the study was stopped after the interim analysis.

Assessment of healing was conducted by investigators blind to treatment allocation.

Participants

134 patients randomised at 16 study centres (German medical practices specialising in phlebology and German and Dutch phlebology outpatient clinics).

Inclusion criteria: venous ulcer, WIDMER stage III, CEAP 6; breadth 1-10 cm; baseline ulcer duration < 12 months; reflux of extrafascial cutaneous saphenous veins or deep conducting veins or perforating veins confirmed by Doppler or Duplex sonography; ABPI > 0.9; patient age 18-80 years.

Exclusion criteria: ambulatory $<1 \mathrm{~h}$ /day; ulcer clinically infected; ulcers of diabetic, arterial or combined aetiology; insulin-dependent diabetes mellitus; diabetic polyneuropathy; DVT in last 3 months; uncontrolled hypertension; advanced coronary disease; primary chronic polyarthritis; ankle dorsal flexion $<5^{\circ}$; vascular surgery or sclerotherapy within last 3 months; concomitant venous medication, immunosuppressants or cytostatics; BMI > $35 \mathrm{~kg} / \mathrm{m}^{2}$; general risk factors; non-compliance.

All patients were Caucasian.

Number of male:female patients: Group 1: 21:40; Group 2: 26:34.

Mean \pm SD patient age in years: Group 1: $63 \pm 11$; Group 2: $63 \pm 13$.

Mean \pm SD BMI in kg/m²: Group 1: $28 \pm 4$; Group 2: $28 \pm 5$.

Mean \pm SD, median baseline ulcer surface area in $\mathrm{mm}^{2}$ : Group 1: $562 \pm 788,274$; Group 2: $595 \pm 899$, 370 .

Mean \pm SD baseline ulcer duration in days: Group 1: $116 \pm 100$; Group 2: $156 \pm 120$.

Number (\%) patients with diabetes: Group 1: 7/61 (11\%); Group 2: 7/60 (12\%).

Number (\%) patients who had compression prior to study: Group 1: 54/61 (89\%); Group 2: 54/60 (90\%).

Interventions

All patients given instructions and written information on how to apply their respective compression system. Compression therapy to be applied for at least $8 \mathrm{~h} /$ day. Patients could reapply compression between clinic visits, or could request professional assistance.

Group 1: U-Stocking (Venotrain ulcertec), consisting of outer and inner stockings, with size specified individually for each patient ( 3 ready-made widths available, each in 2 lengths) $(n=66)$.

Mean \pm SD ankle pressure of $\mathrm{U}$-Stocking measured while supine was $42.7 \pm 13.0 \mathrm{mmHg}$.

Group 2: compression bandages (2 SSBs each $10 \mathrm{~cm}$ wide and $5 \mathrm{~m}$ long, wrapped around leg in opposite directions from metatarsophalangeal joint to the head of the fibula) $(n=68)$.

Ankle pressure not reported for compression bandages.

Outcomes

Number (\%) patients with complete healing at 12 weeks: Group 1: 29/61 (47.5\%); Group 2: 19/60

$(31.7 \%)(95 \% \mathrm{Cl}$ for between-group differences weighted by centre $4.3 \%-28.5 \%$, one-sided $\mathrm{P}=0.013)$.

Mean \pm SD, median (range) days to healing: Group 1: $46 \pm 20,47$ (10-83); Group 2: $46 \pm 22,52$ (6-80), $P=$ 0.82 (Mann-Whitney U-test).

Kaplan-Meier survival analysis indicated a trend in favour of Group 1: ( $P=0.057$, log rank test). Cumulative proportions of patients healed at 12 weeks as read from survival plot: Group 1: 51\%; Group 2: 30\%.

Mean \pm SD, median (range) \% change in ulcer surface area at 12 weeks: Group 1: $(n=61)-74.8 \pm 42.4$, -98.4 (-100-83); Group 2: $(n=58)-51.4 \pm 86.7,-82.9(-100-396.2), P=0.068$ (Mann-Whitney U-test).

Mean \pm SD, median duration of compression therapy (h/day) assessed during the trial Group 1: $12.7 \pm$ 2.9, 12.2; Group 2: $16.9 \pm 5.7,15.9(P=0.0002)$.

Number (\%) patients reporting difficulty in application of compression device (mild vs moderate vs great):

Group $1(n=54): 11(20 \%)$ vs $4(7 \%)$ vs $2(4 \%)$; Group $2(n=53): 12(23 \%)$ vs $6(11 \%)$ vs $0(0 \%)(P=0.9$, chi-squared test). 
Number of adverse events: Group 1: 29 adverse events in 20/65 (31\%) patients; Group 2: 42 adverse events in 26/67 (39\%) patients.

Number of serious adverse events: Group 1: 2 serious adverse events, both resulting in discontinuation of study treatment (ulcer bleeding/pain 1, gastrointestinal bleeding 1); Group 2: 4 serious adverse events (ulcer bleeding 1 , lymph secretion from ulcer 1 , fractured neck of femur - discontinued treatment 1 , thrombophlebitis - discontinued treatment 1 ).

Number of non-serious adverse events: Group 1: 2 non-serious adverse events (increased ulcer pain 1 , increase in calf circumference and open sites around ulcer - treatment discontinued 1); Group 2: 4 non-serious adverse events (ulcer increased in size 1 , ankle flexibility restricted by pain 1 , intolerance to compression material leading to discontinuation of treatment 1 , phlegmon on lower leg - treatment discontinued 1).

Patient questionnaire on comfort of compression showed significantly more patients reporting no problems in Group 1 for: constriction $(P=0.003)$; restricted freedom of movement $(P=0.0009)$; sweating under dressing $(P=0.04)$; and itching of skin on leg $(P=0.006)$. There were no significant between-group differences for tightness, leg pain, burning in leg, heat sensation in leg and prickling of leg.

Mean \pm SD, median ( $25 \%$ and $75 \%$ quartiles) minutes taken for nurse to apply compression: Group 1: $5.4 \pm 5.4,3$ ( 2 and 5); Group 2: $8.5 \pm 6.5,6$ (5 and 10), $P<0.001$.

Number (\%) patients receiving professional support for bandage application: Group 1: 6/65 (9.2\%); Group 2: $15 / 67(22.4 \%), P=0.065$.

Cost analysis based on cost of procedures and associated resources, including: application of stockings or bandages; primary dressings (moist or gauze); debridement (enzymatic or surgical); skin care with zinc paste; skin treatment with topical corticosteroids; physiotherapy; and lymphatic drainage. Labour costs included; overhead costs excluded. The number and type of procedures were patient-reported. Estimated cost/\% reduction in wound area (EUR, price year 2003): Group 1: EUR 2.57; Group 2: EUR 4.58.

Notes

Largest wound studied in patients with multiple ulcers. Group 2 ulcers larger and more chronic at baseline. Main analysis should be regarded as the Kaplan-Meier survival analysis: between-group difference in time to healing was tested using the Mann-Whitney U-test but the log rank test would have been preferable.

Ulcer surface areas estimated using a digital image of the wound perimeter traced onto foil combined with computerised planimetry. The calculation was performed at a central research office by a technician blind to treatment allocation. Ulcers were photographed.

Withdrawals/exclusions from analysis: following randomisation 1 patient/group excluded (Group 1: additional thigh compression needed prior to start of study treatment; Group 2: refused treatment prior to start of therapy). Safety analysis based on: Group 1: $n=65$; Group 2: $n=67.11$ of these patients withdrew early and had no efficacy data: Group 1: $n=4$ (serious adverse event 2, ineligible 2); Group 2: $n=$ 7 (serious adverse event 2, ineligible 2, lost to follow-up 3). The intention-to-treat population available for the primary efficacy analyses was based on: Group 1: $n=61$; Group 2: $n=60.12$ of these patients withdrew after at least 1 post-baseline assessment: Group $1: n=6$ (withdrawal of consent 4, poor compliance 2); Group 2: $\mathrm{n}=6$ (withdrawal of consent 2, poor compliance 2, adverse events 2).

The authors stated that: the bandaging method used for Group 2 was standardised in all study centres; all persons involved in providing nursing care were given training in applying compression.

\section{Risk of bias}

\begin{tabular}{lll}
\hline Bias & Authors' judgement & Support for judgement \\
\hline $\begin{array}{l}\text { Random sequence genera- } \\
\text { tion (selection bias) }\end{array}$ & Unclear risk & $\begin{array}{l}\text { "Randomization used blocks of } 4 \text { patients and was performed at the statisti- } \\
\text { cal department of a contract research organisation ... prior to patient enrol- } \\
\text { ment". }\end{array}$ \\
\hline
\end{tabular}


Jünger 2004b (Continued)

Allocation concealment Low risk_ "Numbered containers were supplied to the study sites; patients were as(selection bias)

\section{signed by the investigators to one of the two treatments by opening a code en-} velope with available treatment numbers in ascending order".

\begin{tabular}{lll}
\hline $\begin{array}{l}\text { Incomplete outcome data } \\
\text { (attrition bias) } \\
\text { All outcomes }\end{array}$ & High risk & $\begin{array}{l}134 \text { patients were randomised and } 121 \text { were analysed; } 6 \text { people withdrew from } \\
\text { each group. }\end{array}$ \\
\hline $\begin{array}{l}\text { Blinded outcome assess- } \\
\text { ment (healing) }\end{array}$ & Low risk & $\begin{array}{l}\text { The calculations of ulcer surface area were performed at a central research of- } \\
\text { fice by a technician blind to treatment allocation. }\end{array}$ \\
\hline
\end{tabular}

Baseline comparability High risk Possible imbalances for ulcer area (median ulcer larger in Group 2 and also mean duration longer in Group 2).

\title{
Kikta 1988
}

Methods RCT (no further details of methods of allocation). Setting, outpatients, USA.

\section{Participants}

\begin{abstract}
84 patients with 87 leg ulcers caused by chronic venous insufficiency recruited from hospital vascular surgery clinics.

Exclusion criteria: arterial insufficiency (ABPI < 0.7); uncontrolled diabetes mellitus; use of cancer chemotherapeutic agents or systemic steroids; recent venous surgery; infected ulcers; inability to comply with treatment or follow-up.

Mean \pm SEM baseline ulcer area in $\mathrm{cm}^{2}$ : Group 1: $9.0 \pm 2.2$; Group 2: $8.6 \pm 2.1$.

Mean \pm SEM baseline ulcer duration in weeks: Group 1: $51 \pm 17$; Group 2: $45 \pm 12$.

Authors reported that groups were comparable for other baseline variables including: patient age; sex; race; previous ulcer treatment; pre-randomisation use of antibiotics; origin of chronic venous insufficiency; previous venous, arterial or orthopaedic surgery; prior use of elastic stockings; ischaemic heart disease; congestive heart failure; obesity; hypertension; diabetes mellitus; pulmonary, renal and hepatic diseases; use of oral contraceptives or tobacco; alcoholism; elevated levels of serum haemoglobin, glucose, albumin and creatinine; ABPI; and whether ulcer was new or recurrent. Data were not presented for these variables. The source population was described as "inner city, lower socioeconomic class".
\end{abstract}

Interventions

All patients received instructions about leg elevation, restriction of standing activities, care of associated medical problems, and importance of compliance and follow-up. At each clinic visit, ulcers were washed with dilute chlorhexidine solution followed by $3 \% \mathrm{H}_{2} \mathrm{O}_{2}$, rinsed with normal saline and left to air dry.

Group 1: Unna's boot (further details of components not provided ( $n=42$ ulcers); Group 2: Duoderm hydrocolloid dressing (no compression applied) ( $\mathrm{n}=45$ ulcers).

Outcomes Analysis based on 66 patients with 69 ulcers: Group 1: $n=30$ ulcers; Group 2: $n=39$ ulcers.

Number (\%) of ulcers completely healed at 6 months: Group 1: 21/30 (70\%); Group 2: 15/39 (38\%) (P= 0.01 , chi-squared test).

Lifetable analysis of mean \pm SEM proportion of ulcers healed at 15 weeks: Group 1: $64 \% \pm 9 \%$; Group 2: $35 \% \pm 8 \%(P=0.01, \log$ rank test $)$.

Mean \pm SEM time to healing in weeks: Group 1: $8.4 \pm 1.8$; Group 2: $7.0 \pm 1.5(P=0.8$, Student's t-test).

Findings from logistic regression suggested that the following were significant predictors of healing: dressing type $(P=0.002)$; and baseline ulcer area $(P=0.04)$. Other covariates that were tested, but did not emerge as significant predictors included: baseline ulcer duration, patient age, sex, race, obesity and diabetes.

Number (\%) ulcers withdrawn from study (all withdrew within 2 weeks of randomisation, reasons not provided): Group 1: 12/42 (29\%); Group 2: 6/45 (13\%) ( $P=0.11$, Fishers' exact test). 
Kikta 1988 (Continued)

Number (\%) of ulcers with adverse events resulting in discontinuation of treatment: Group 1: 0/30 (0\%); Group 2: 10/39 (26\%) (8 developed reddish-green exudate, 2 had associated cellulitis requiring hospital admission). $\mathrm{P}=0.004$ for difference between groups (Fisher's exact test).

Mean \pm SEM pain score evaluated by patients post-healing using linear scale $1-10$ (meaning of values not explained): Group 1: $2.4 \pm 0.4$; Group 2: $1.2 \pm 0.1$ ( $P=0.007$, Student's t-test).

Mean \pm SEM cost of treatment/week in USD (price year 1986) based on cost of all dressing materials divided by time to healing (healed ulcers) or duration of therapy (non-healed ulcers). Clinic visit costs and staff costs were excluded: Group 1: USD $11.76 \pm 0.59$; Group 2: USD $14.24 \pm 1.63(P=0.16$, Student's ttest).

\section{Notes}

Ulcer area measured using tracing and computerised planimetry. Dressings applied according to manufacturers' instructions.

\section{Risk of bias}

\begin{tabular}{|c|c|c|}
\hline Bias & Authors' judgement & Support for judgement \\
\hline $\begin{array}{l}\text { Random sequence genera- } \\
\text { tion (selection bias) }\end{array}$ & Unclear risk & "Patients with leg ulcers ... were randomised to receive ... ". \\
\hline $\begin{array}{l}\text { Allocation concealment } \\
\text { (selection bias) }\end{array}$ & Unclear risk & "Patients with leg ulcers ... were randomised to receive ... ". \\
\hline $\begin{array}{l}\text { Incomplete outcome data } \\
\text { (attrition bias) } \\
\text { All outcomes }\end{array}$ & High risk & 84 people were randomised, however, only 66 were analysed. \\
\hline $\begin{array}{l}\text { Blinded outcome assess- } \\
\text { ment (healing) }\end{array}$ & Unclear risk & $\begin{array}{l}\text { "Ulcer size was measured by tracing the ulcer outline and then measuring the } \\
\text { area with a computerised digital planimeter." }\end{array}$ \\
\hline Baseline comparability & Unclear risk & $\begin{array}{l}\text { Appear similar for baseline area and duration (however only means present- } \\
\text { ed). Authors reported that groups were comparable for other baseline vari- } \\
\text { ables including: patient age; sex; race; previous ulcer treatment; pre-randomi- } \\
\text { sation use of antibiotics; origin of chronic venous insufficiency; previous ve- } \\
\text { nous, arterial or orthopaedic surgery; prior use of elastic stockings; ischaemic } \\
\text { heart disease; congestive heart failure; obesity; hypertension; diabetes melli- } \\
\text { tus; pulmonary, renal and hepatic diseases; use of oral contraceptives or to- } \\
\text { bacco; alcoholism; elevated levels of serum haemoglobin, glucose, albumin } \\
\text { and creatinine; ABPI; and whether ulcer was new or recurrent. Data not pre- } \\
\text { sented for these variables. }\end{array}$ \\
\hline
\end{tabular}

\section{Knight 1996}

Methods $\quad$ RCT (no further details of methods). Setting was a wound care centre in the USA.

Participants
Inclusion criteria: venous insufficiency (not defined); leg ulcer of venous aetiology.
Exclusion criteria: refused consent.
No information provided about baseline characteristics except venous filling index.

Interventions $\quad$ Group 1: 4LB (Profore) $(\mathrm{n}=5$ patients);

Group 2: Unna's boot (described as a paste-impregnated gauze compression dressing) ( $n=5$ patients).

All patients received a foam dressing (Allevyn) as the primary dressing. Dressings and bandages changed weekly. 
Knight 1996 (Continued)

Outcomes

At 6 weeks: Mean \pm SD healing rate in $\mathrm{cm}^{2}$ per week: Group $11.139 \pm 0.931$; Group $20.339 \pm 0.458$.

These values were calculated by the reviewer using raw data from the study report.

Notes

Few details of this trial were available. Data were extracted from a conference abstract and a brief, unpublished report provided by the trial authors. Ulcer surface area assessed weekly using transparency tracing and computerised planimetry. Patients followed-up for 6 weeks. Venous filling index, measured by air plethysmography, reported at baseline, day 1 and day 7 . This study is described as ongoing, but no follow-up reports have been identified.

\section{Risk of bias}

\begin{tabular}{|c|c|c|}
\hline Bias & Authors' judgement & Support for judgement \\
\hline $\begin{array}{l}\text { Random sequence genera- } \\
\text { tion (selection bias) }\end{array}$ & Unclear risk & "... subjects for the study are randomly assigned ... ". \\
\hline $\begin{array}{l}\text { Allocation concealment } \\
\text { (selection bias) }\end{array}$ & Unclear risk & No details provided. " . . subjects for the study are randomly assigned ... ". \\
\hline $\begin{array}{l}\text { Incomplete outcome data } \\
\text { (attrition bias) } \\
\text { All outcomes }\end{array}$ & Unclear risk & $\begin{array}{l}10 \text { patients recruited; data on } 10 \text { participants. This trial was ongoing at time of } \\
\text { trial report, but no further data received. }\end{array}$ \\
\hline $\begin{array}{l}\text { Blinded outcome assess- } \\
\text { ment (healing) }\end{array}$ & Unclear risk & $\begin{array}{l}\text { Wounds were measured by transparency tracing combined with computerised } \\
\text { planimetry, but unclear how these images were assessed and whether ob- } \\
\text { servers were blinded. }\end{array}$ \\
\hline Baseline comparability & Unclear risk & No baseline data presented. \\
\hline
\end{tabular}

\section{Koksal 2003}

\begin{tabular}{|c|c|}
\hline Methods & RCT (method of randomisation not stated). Setting: university Hospital Clinic, Turkey. \\
\hline \multirow[t]{5}{*}{ Participants } & $\begin{array}{l}60 \text { outpatients. Average (range) age in years: Group 1: } 51 \text { (24-70); Group 2: } 49 \text { (20-72). } \\
\text { Inclusion criterion: venous leg ulceration on gaiter area (diagnosed clinically) with area } 5-8 \mathrm{~cm}^{2} \text {. } \\
\text { Exclusion criteria: } \mathrm{ABPI}<0.8 \text {; clinical infection requiring treatment; diabetes; causes of leg ulceration } \\
\text { other than venous. }\end{array}$ \\
\hline & Number of male:female patients: Group 1: 9:21; Group 2: 11:19. \\
\hline & Previous ulcer recurrence: Group 1: 74\%; Group 2: 73\%. \\
\hline & Mean \pm SD baseline ulcer area $\left(\mathrm{cm}^{2}\right)$ : Group 1: $6.38 \pm 1.2 ;$ Group 2: $6.19 \pm 0.8$ \\
\hline & Mean \pm SD baseline ulcer duration (weeks): Group 1: $16.6 \pm 5.8$; Group 2: $16.9 \pm 6.2$ \\
\hline \multirow[t]{4}{*}{ Interventions } & $\begin{array}{l}\text { Concurrent treatments: all ulcers cleansed with normal saline and debrided (no further details of } \\
\text { agents used), when necessary. }\end{array}$ \\
\hline & $\begin{array}{l}\text { Group 1: Unna's Boot containing calamine, zinc oxide, glycerine, sorbitol, gelatine and magnesium alu- } \\
\text { minium silicate }(n=30) \text {. }\end{array}$ \\
\hline & $\begin{array}{l}\text { Group 2: hydrocolloid dressing (Comfeel) plus class II elastic compression stocking providing } 30-40 \mathrm{Hg} \text { - } \\
\mathrm{mm}(\mathrm{n}=30) \text {. }\end{array}$ \\
\hline & Dressings changed every 3-7 days. \\
\hline
\end{tabular}


Koksal 2003 (Continued)

Outcomes
Ulcer area measured by transparency tracing and planimetry (instrument not stated). Areas calculated by an investigator blind to treatment allocation.

Patients with complete healing at 4 months: Group 1: 20/27 (74\%); Group 2: 21/26 (81\%), P > 0.05 .

Mean \pm SD healing rate $\left(\mathrm{cm}^{2}\right.$ per week): Group 1: $1.28 \pm 0.72$; Group 2: $1.16 \pm 0.38, P>0.05$.

Mean \pm SD weeks to healing: Group 1: $6.85 \pm 3.60$; Group 2: $6.65 \pm 3.31, P>0.05$.

Mean \pm SD pain score during application (measured with visual analogue scale $0-10$, where $0=$ no pain and $10=$ worst imaginable pain): Group 1: $3.69 \pm 1.35$; Group 2: $1.88 \pm 1.48, \mathrm{P}<0.0001$.

Mean \pm SD pain score at home (measured as above): Group 1: $3.27 \pm 1.08$; Group 2: $1.88 \pm 1.11, \mathrm{P}<$ 0.0001 .
Unclear whether patients in Group 2 removed stockings when going to bed. Concerning skill of care provider, the paper reported that "two dedicated and trained outpatient nurses applied both treatment modalities".

Withdrawals: Group 1: 3 (infected ulcers 2, hospitalised 1); Group 2: 4 (infection 1; severe reaction to dressing 1 ; did not attend clinic 1 ; lost to follow-up 1 ).

No patient experienced a serious adverse event during the trial. One treatment-related adverse event reported in Group 2:

\section{Risk of bias}

\begin{tabular}{lll}
\hline Bias & Authors' judgement & Support for judgement \\
\hline $\begin{array}{l}\text { Random sequence genera- } \\
\text { tion (selection bias) }\end{array}$ & Unclear risk & "The patients were randomly assigned into two groups". \\
\hline $\begin{array}{l}\text { Allocation concealment } \\
\text { (selection bias) }\end{array}$ & Unclear risk & "The patients were randomly assigned into two groups". \\
\hline $\begin{array}{l}\text { Incomplete outcome data } \\
\text { (attrition bias) } \\
\text { All outcomes }\end{array}$ & High risk & $\begin{array}{l}60 \text { patients recruited and complete healing analysis based on 53 participants. } \\
\text { Denominator unclear for continuous outcomes. 3 patients withdrew from } \\
\text { Group 1, and 4 from Group 2. }\end{array}$ \\
\hline $\begin{array}{l}\text { Blinded outcome assess- } \\
\text { ment (healing) }\end{array}$ & Low risk & $\begin{array}{l}\text { Ulcer tracings and planimetry performed by a technician who was unaware of } \\
\text { the treatment allocation. }\end{array}$ \\
\hline $\begin{array}{l}\text { Baseline comparability } \\
\text { Unclear risk }\end{array}$ & $\begin{array}{l}\text { Mean ulcer area and duration were similar, however, medians were not pre- } \\
\text { sented and data are likely to be skewed. }\end{array}$ \\
\hline
\end{tabular}

Kralj 1996

Methods RCT (open design). Setting included both in-patients and out-patients in Slovenia.

\footnotetext{
Participants

40 patients recruited.

Inclusion criteria: stasis leg ulcer, age < 86 years, complete mobility, written, informed consent.

Exclusion criteria: $\mathrm{ABPI}<0.8$, systemic connective tissue disease, serological positive rheumatoid arthritis, severe concurrent diseases.

Number of male:female patients: Group 1: 6:10; Group 2: 8:10.

Mean (range) patient age in years: Group 1: 65 (40-86); Group 2: 61 (36-85).

Mean (range) baseline ulcer area in $\mathrm{cm}^{2}$ : Group 1: 18.6 (1-57); Group 2: 17.2 (1-47).

Mean (range) duration of ulcers in months: Group 1: 7.9 (1-24); Group 2: 6.9 (1-36).
} 
Kralj 1996 (Continued)

Interventions
Group 1: 4LB (Profore): wool, crepe, Litepress, Co-Plus ( $\mathrm{n}=20$ patients).

Group 2: hydrocolloid dressing (Tegasorb) and single layer inelastic bandage (Porelast) $(\mathrm{n}=20$ patients).

Bandages were changed at least weekly for all patients.

$\begin{array}{ll}\text { Outcomes } & \text { Number (\%) patients with complete healing during 6-month trial (NB patients started treatment at dif- } \\ \text { ferent points within this 6-month period): Group 1: 7/20 (35\%); Group 2: 8/20 (40\%). } \\ \text { Mean (range) days to healing: Group 1: } 57.6 \text { ( 7-106); Group 2: 84.9 (28-180). } \\ \text { Number (\%) patients withdrawing from trial (reasons): Group 1: 4/20 (20\%) (admitted to hospital with } \\ \text { heart condition 1, no transport to clinic 1, unknown reason 2); Group 2: 2/20 (10\%) (cerebrovascular } \\ \text { apoplexy 1, unknown reason 1). } \\ \text { Maximum length and width of ulcer measured at each bandage change. Ulcer surface area calculated } \\ \text { Notes } \\ \text { multiple ulcers, the total ulcerated area was studied. Study described as ongoing. }\end{array}$

\section{Risk of bias}

\begin{tabular}{|c|c|c|}
\hline Bias & Authors' judgement & Support for judgement \\
\hline $\begin{array}{l}\text { Random sequence genera- } \\
\text { tion (selection bias) }\end{array}$ & Unclear risk & $\begin{array}{l}\text { Communication with trialists confirmed that randomisation was by sealed en- } \\
\text { velope, but not clear if opaque or numbered. Method of sequence generation } \\
\text { unclear. }\end{array}$ \\
\hline $\begin{array}{l}\text { Allocation concealment } \\
\text { (selection bias) }\end{array}$ & Unclear risk & $\begin{array}{l}\text { Communication with trialists confirmed that randomisation was by sealed en- } \\
\text { velope, but not clear if opaque or numbered. }\end{array}$ \\
\hline $\begin{array}{l}\text { Incomplete outcome data } \\
\text { (attrition bias) } \\
\text { All outcomes }\end{array}$ & High risk & $\begin{array}{l}40 \text { patients recruited; } 4 \text { people withdrew from Group } 1 \text { and } 2 \text { from Group 2: } \\
\text { These people were not included in the analysis. }\end{array}$ \\
\hline $\begin{array}{l}\text { Blinded outcome assess- } \\
\text { ment (healing) }\end{array}$ & Unclear risk & "Wounds were assessed by authors... " (personal correspondence). \\
\hline Baseline comparability & Unclear risk & $\begin{array}{l}\text { Mean ulcer areas and durations similar, but not very informative, since data } \\
\text { skewed. }\end{array}$ \\
\hline
\end{tabular}

\section{Mariani 2008}

\begin{tabular}{ll}
\hline Methods & RCT performed at 3 specialist leg ulcer care centres in Italy (Siena, Lucca and Ferrara). Trial authors \\
stated that there was no stratification at randomisation. Block randomisation was used ( 2 blocks of 10 \\
patients/centre).
\end{tabular}

\section{Participants}

60 patients recruited from specialist leg ulcer care centres.

Inclusion criteria: presence of venous leg ulcer confirmed by clinical examination and duplex ultrasound; maximum ulcer diameter $8 \mathrm{~cm}$, minimum duration 1 month; no effective compression treatment prior to trial; patient able and willing to follow study protocol.

Exclusion criteria: effective compression therapy started prior to trial; arterial insufficiency (defined as non-palpable foot pulse and $\mathrm{ABPI}<0.8$ ); neuropathy of diabetic or other origin; varicose vein or ulcer surgery within 3 months of enrolment; acute DVT or varico-thrombosis requiring anticoagulation; ulcer of dermatological cause; primary lymphoedema; pregnancy; life expectancy < 90 days.

Baseline data apply to 56/60 patients: 
Number of male:female patients: Group 1: 13:13; Group 2: 10:20.

Mean \pm SD patient age in years: Group 1: $62.4 \pm 14.0$; Group 2: $65.2 \pm 15.3$.

Mean \pm SD ulcer diameter in cm: Group 1: $3.38 \pm 2.73$; Group 2: $2.76 \pm 2.37$.

Mean \pm SD ulcer duration in months: Group 1: $3.2 \pm 2.96$; Group 2: $3.6 \pm 4.01$.

Number of patients with primary vs recurrent ulceration: Group 1: 22 vs 4; Group 2: 24 vs 6.

Pain: absent vs weak vs moderate vs strong: Group 1: 2 vs 10 vs 12 vs 2; Group 2: 0 vs 15 vs 9 vs 6 .

Interventions

Group 1: 2-layer compression stocking kit (Sigvaris ${ }^{\circledast}$ Ulcer $X^{\circledR}$ kit). Understocking consisted of a fabric with an inner cotton layer and an outer knitted layer. Top stocking consisted of double-covered natural rubber (information gleaned from manufacturer's brochure; this stocking in isolation has the proprietary name Sigvaris ${ }^{\circledast}$ Traditional $\left.^{\circledR}\right)$. Sub-stocking ankle pressure with both layers in place was designed to be $39 \mathrm{mmHg}$. Top stocking removed at night. Kit washed every 2 days on average ( $\mathrm{n}=30$ patients).

Group 2: SSB applied with spiral or figure-of-8 application, worn day and night ( $n=30$ patients).

All patients: planned to assess all patients weekly with longer or shorter intervals allowed according to patient need. Primary dressings and other topical treatments were applied according to the judgement of treating physician. Surgery was not permitted during the trial.

Outcomes

Number (\%) of patients with complete healing at 4 months: Group 1: 25/30 (83\%); Group 2: 21/30 (70\%).

Mean \pm SD time to healing in days: Group 1: $56.0 \pm 29.1$; Group 2: $61.1 \pm 22.7(P=0.52)$.

Trial authors reported that smaller ulcers healed more rapidly with the stockings, whilst the time to healing of larger ulcers was similar for both treatment groups.

Pain, discomfort and hindrance of activities were assessed with the Venous Leg Ulcer Questionnaire applied at the last clinic visit (either at healing or at 4 months). Mean scores from 5-point Likert scales suggested that the following were significantly worse at the $5 \%$ level for Group 2: inhibition of activities (P $=0.025)$, pain at donning and removal $(P=0.001)$ and number of problems reported $(P<0.0001)$. Mean scores for daytime discomfort and pain suggested no significant difference between treatment groups $(P=0.086)$.

Number (\%) patient withdrawals (reasons): Group 1: 4/30 (13\%) patients excluded within 1st week after randomisation (withdrew consent 1 , had restricted ankle movement and could not put on the stockings 3); Group 2: 0/30 (0\%).

Notes Ulcer diameter measured using a tape at baseline and at each assessment.

Bandages applied by an expert study physician (no other details of staff expertise provided).

Use of stocking kit associated with longer intervals between clinic visits (mean \pm SD $8.2 \pm 1.8$ versus 6.7 $\pm 1.0, P=0.002)$; mean number of clinic visits until healing similar between groups $(P=0.157)$.

\section{Risk of bias}

Bias Authors' judgement Support for judgement

Random sequence genera- Unclear risk tion (selection bias)
"The study is a prospective, randomised, open-label, parallel-group clinical trial...".

"No stratification is done, neither for the size of the ulcer nor its presumed cause. Randomization is done in two blocks of 10 patients for each centre".

From secondary reference: "Sealed envelope technique was used for randomisation". 

domised sequence.

\begin{tabular}{ll}
\hline $\begin{array}{l}\text { Allocation concealment } \\
\text { (selection bias) }\end{array}$ & Unclear risk \\
& $\begin{array}{l}\text { From secondary reference: "Sealed envelope technique was used for randomi- } \\
\text { sation". }\end{array}$ \\
$\begin{array}{l}\text { Comment: it was not clear whether the sealed envelopes were consecutively } \\
\text { numbered and opaque. }\end{array}$
\end{tabular}

Incomplete outcome data High risk
(attrition bias)
"Four patients were excluded within the first week after randomisation, all in the stocking group. One patient refused to continue with any kind of compres- sion therapy. Three patients were unwilling to continue as donning of the sec- ond stocking was too difficult for them."

All outcomes

Comment: withdrawal rate differed across groups (Group 1: 13\% and Group 2: nil), potentially related to treatment in Group 1.

\begin{tabular}{lll}
\hline $\begin{array}{l}\text { Blinded outcome assess- } \\
\text { ment (healing) }\end{array}$ & Unclear risk & $\begin{array}{l}\text { "The study is a prospective, randomised, open-label, parallel-group clinical tri- } \\
\text { al". }\end{array}$ \\
Comment: It was not clear whether the outcome assessment was blinded.
\end{tabular}

\section{Meyer 2002}

$\begin{array}{ll}\text { Methods } & \text { RCT with randomisation by computer-generated tables and stratification by baseline ulcer area. The } \\ \text { strata were }\left(\mathrm{cm}^{2}\right) \text { : small }(0.25-2.5) \text {; medium }(>2.5-25) ; \text { large }(>25-100) \text {. For stratification purposes, ulcer } \\ \text { area was measured using diameter product (multiplication of maximum length and width). A post-hoc } \\ \text { statistical power analysis was presented as part of the discussion section. }\end{array}$

Pecruited 112 patients from a hospital leg ulcer outpatient clinic in the UK.
Number of patients with small vs medium vs large ulcers at baseline: Group 1: 18 vs 23 vs 16; Group 2:
17 vs 23 vs 15.
Exclusion criteria: $\mathrm{ABPI}<0.8$; diabetes; rheumatoid arthritis; systemic lupus erythematosus; positive
sickle cell test; $\mathrm{HIV}$; ulcer size $<0.25 \mathrm{~cm}^{2}$ or $>100 \mathrm{~cm}^{2}$; known sensitivity to Viscopaste; receiving drugs
that might affect ulcer healing; non-venous diagnosis of ulcer on clinical examination; no venous ab-
normality detected using haemodynamic assessment, even if clinical examination indicated venous ae-
tiology.

Interventions Concurrent treatment for both groups: ulcer and surrounding skin cleansed with saline-soaked cotton wool balls. Standardised figure-of-8 technique used for bandaging.

Group 1: Viscopaste bandage plus Tensopress (elastic bandage) plus Tensoshape (graduated cotton-elastic tubular retaining bandage) $(n=57)$.

Group 2: Viscopaste bandage plus Elastocrepe (inelastic bandage) plus Tensoshape (description as above) $(n=55)$.

All dressings undisturbed between clinic visits; frequency of clinic visits not stated.

Outcomes

Number of patients with complete healing (assessed by photograph) at 26 weeks: Group 1: 33/57 (58\%); Group 2: 34/55 (62\%), P = 0.623 (P value generated from Kaplan-Meier estimates and log rank test).

Patients with large ulcers significantly less likely to heal within 26 weeks than those with small or medium-sized ulcers (chi-squared test $=18.05, \mathrm{P}<0.001$ ), and this was independent of treatment effect. 
Further analysis at 40 weeks showed that 1 extra patient/group had healed - this did not affect statistical significance of the between-group difference.

Mean [range] (95\% Cl) weeks to healing: Group 1: 10 [2-23] (8-12); Group 2: 11 [3-25] (9-13), not significant.

Median weeks to healing: Group 1: 9; Group 2: 9.5, not significant.

Notes Unclear whether photographic confirmation of healing done by assessor blind to treatment allocation.

Care providers were "seven experienced ulcer clinic nurses".

Number of patients excluded post-randomisation because ineligible: Group 1: 4; Group 2: 5.

Number of patients who withdrew from treatment during trial (with reasons): Group 1: 8 (bandage skin damage 1 (pretibial skin necrosis), refused treatment 1 , lost to follow-up 3, incomplete data record 3); Group 2: 8 (paste allergy 1; non-compliant 2; lost to follow-up 3; incomplete data record 2).

Costs/bandage: Group 1: GBP 4.38; Group 2: GBP 2.54 (price year not stated).

Mean initial ankle pressures using the Borgnis medical stocking test apparatus: Group 1: $45 \mathrm{mmHg}$; Group 2: $24 \mathrm{mmHg}$.

\section{Risk of bias}

\begin{tabular}{|c|c|c|}
\hline Bias & Authors' judgement & Support for judgement \\
\hline $\begin{array}{l}\text { Random sequence genera- } \\
\text { tion (selection bias) }\end{array}$ & Low risk & "Computer generated tables were used to randomise patients". \\
\hline $\begin{array}{l}\text { Allocation concealment } \\
\text { (selection bias) }\end{array}$ & Unclear risk & No further details provided. \\
\hline $\begin{array}{l}\text { Incomplete outcome data } \\
\text { (attrition bias) } \\
\text { All outcomes }\end{array}$ & Low risk & $\begin{array}{l}112 \text { people randomised and } 112 \text { analysed for complete healing, however, oth- } \\
\text { er outcomes unclear. }\end{array}$ \\
\hline $\begin{array}{l}\text { Blinded outcome assess- } \\
\text { ment (healing) }\end{array}$ & Unclear risk & No detail provided. \\
\hline Baseline comparability & Unclear risk & $\begin{array}{l}\text { Randomisation stratified by ulcer area at baseline, however, neither mean nor } \\
\text { median ulcer area presented by group. }\end{array}$ \\
\hline
\end{tabular}

Meyer 2003

Methods

RCT with randomisation by computer-generated tables and stratification by baseline ulcer area. The strata were $\left(\mathrm{cm}^{2}\right)$ : small (0.25-2.5); medium (>2.5-25); large (>25-100). For stratification purposes, ulcer area measured using diameter product (i.e. multiplication of maximum length and width). For patients with bilateral ulcers, the combined area of the ulcers on both legs was used for stratification. Using an a priori power calculation, it was estimated that the study had 50\% power to detect a difference of $20 \%$ in frequency of complete healing at the $95 \%$ significance level.

Participants

Recrtuied 133 patients from a hospital leg ulcer outpatient clinic in the UK.

Number of male:female patients: Group 1: 34:30; Group 2: 41:28.

Median age in years: Group 1: 68 Group 2: 64.

Mean duration of ulcer in months: Group 1: 19.8 Group 2: 14.8. 
Number of patients with small vs medium vs large ulcers at baseline: Group 1: 25 vs 18 vs 21 Group 2: 21 vs 21 vs 27 .

Exclusion criteria: $\mathrm{ABPI}<0.9$; diabetes; rheumatoid arthritis; systemic lupus erythematosus; positive sickle cell test; HIV; ulcer size $<0.25 \mathrm{~cm}^{2}$ or $>100 \mathrm{~cm}^{2}$; known sensitivity to paste; ulcer not of venous aetiology; failure to comply with exit investigations.

Interventions

Concurrent treatment for both groups: ulcer and surrounding skin cleansed with saline-soaked cotton wool balls. Standardised figure-of-8 technique used for bandaging.

Group 1: 3-layer bandage consisting of: Steripaste bandage plus Setopress bandage plus Tubgrip bandage $(n=64)$.

Group 2: 4LB consisting of: Velband orthopaedic wool; crepe bandage; Elset compression bandage; Coban bandage $(n=69)$.

All dressings left undisturbed between clinic visits; frequency of clinic visits initially weekly, then extended to fortnightly in patients deemed to be making good progress in terms of healing.

Outcomes

Patients followed-up to healing or until 52 weeks.

Number of patients with complete healing (assessed by photograph) at 52 weeks: Group 1: 51/64 (80\%); Group 2: 45/69 (65\%), $P=0.031$.

Median (95\% Cl) weeks to healing: Group 1: 12 (10-15); Group 2: 16 (13-21), P = 0.04.

Kaplan-Meier survival estimates showed that the difference in probability of healing between the 2 bandages did not become apparent until 20 weeks after randomisation, $\mathrm{P}=0.036$ (log rank test). The authors reported that this estimate remained robust when the analysis was repeated selecting only patients with venous ulceration confirmed with haemodynamic assessment at completion or withdrawal, but full details not shown in the paper.

Authors stated that ulcer duration did not influence healing, but patients with large ulcers were significantly less likely to heal than those with small or medium ulcers, this effect being independent of treatment (full details of these analyses not shown).

Scores for bandage comfort, pain on bandaging and ease of putting on shoes over bandages (all assessed using an un-validated scale of 1-4 at each visit) increased over the study period, indicating improvement for both groups, but no significant differences were detected between groups.

Group 1 contained significantly more patients with post-thrombotic calf veins; this was assessed at completion or withdrawal using ascending phlebography (full details of analysis not shown).

Notes

Number of patients who withdrew from treatment during trial (with reasons): Group 1: 10 (adverse events 4, non-compliant 2, lost to follow-up 3, refused treatment 1); Group 2: 11 (adverse events 2, noncompliant 5, lost to follow-up 3, refused treatment 1 ).

Unclear whether photographic confirmation of healing done by assessor blind to treatment allocation.

Care providers described as "seven experienced ulcer clinic nurses" and as "dedicated nursing staff who are fully trained in four-layer bandaging".

Statistical calculations performed by 2 statisticians who were independent of one another and of the study investigators.

\section{Risk of bias}

Authors' judgement Support for judgement

Random sequence genera- Low risk Computer generated tables were used to randomise patients.

tion (selection bias) 
Meyer 2003 (Continued)

$\begin{aligned} & \text { Allocation concealment } \\ & \text { (selection bias) }\end{aligned} \quad$ Unclear risk $\quad$ No further detail provided.

\begin{tabular}{lll}
\hline $\begin{array}{l}\text { Incomplete outcome data } \\
\text { (attrition bias) } \\
\text { All outcomes }\end{array}$ & Low risk & $\begin{array}{l}\text { 133 people randomised and complete healing data provided for 133 partici- } \\
\text { pants; unclear for other outcomes. } 10 \text { people withdrew from Group } 1 \text { and } 11 \\
\text { from Group 2. }\end{array}$ \\
\hline $\begin{array}{l}\text { Blinded outcome assess- } \\
\text { ment (healing) }\end{array}$ & Unclear risk & $\begin{array}{l}\text { Unclear whether photographic confirmation of healing was done by an asses- } \\
\text { sor blinded to treatment allocation. }\end{array}$ \\
\hline Baseline comparability & Unclear risk & $\begin{array}{l}\text { Randomisation was stratified by ulcer area at baseline, however, neither mean } \\
\text { nor median ulcer area by group presented. }\end{array}$
\end{tabular}

Milic 2007

Methods with computer-generated randomisation. Some details of sample size calculation provided (80\%
power, significance level 5\%), but unclear what intended clinical difference was expected to be detect-
ed.

Participants

150 patients recruited.

Inclusion criteria: patient age $\geq 18$ years; venous leg ulceration diagnosed using ABPI assessment and colour duplex ultrasonography; baseline ulcer surface area $>20 \mathrm{~cm}^{2}$; baseline ulcer duration $>6$ months.

Exclusion criteria: $\mathrm{ABPI}<0.8$; causes of ulceration other than venous; heart failure (ejection fraction < 35); pregnancy; cancer; diabetes.

Number of male:female patients: Group 1: 39:36; Group 2: 34:41.

Median (range) patient age in years: Group 1: 55 (33-80); Group 2: 57 (34-81).

Median (range) number of previous episodes of ulceration: Group 1: 5 (2-10); Group 2: 5 (1-11).

Median (range) baseline ulcer surface area (cm²): Group 1: 72 (24-210); Group 2: 64 (20-195).

Median (range) baseline ulcer duration in years: Group 1: 7 (0.6-28); Group 2: 6 (0.6-21).

Number (\%) patients with previous DVT: Group 1: 25/72 (35\%); Group 2: 20/66 (30\%).

Number (\%) patients who had previously undergone stripping of great saphenous vein: Group 1: 14/72

(19\%); Group 2: $12 / 66(18 \%)$.

Number (\%) patients who had previously undergone superficial endoscopic perforator vein surgery:

Group 1: 5/72 (7\%); Group 2: 5/66 (8\%).

Authors reported that groups were similar at baseline for CEAP classification.

None of the patients had previously received compression.

All patients: treated on an ambulatory basis and had mechanical debridement using sterile gauze. Dressings changed every 1-7 days, depending on exudate. Extensive exudation treated with crystal acidum boricum applied to the wound following debridement. In cases of no exudate, a dry dressing was applied. Bandage systems were worn day and night. No antibiotics were used. All patients received aspirin (100 $\mathrm{mg}$, we presume this was a daily dose).

Group 1: cotton gauze without tension ( $50 \%$ overlap) plus cotton crepe bandage plus knee-length tubular compression device (Tubulcus) providing $35-40 \mathrm{~mm} \mathrm{Hg}$ at ankle plus medium-stretch elastic compression bandage (Niva). After healing, patients continued to wear Tubulcus $(n=75)$.

Group 2: cotton gauze without tension (50\% overlap) plus cotton crepe bandage plus 2 medium stretch elastic compression bandages (Niva). After healing, patients wore class II compression stockings providing $20-25 \mathrm{~mm} \mathrm{Hg}$ (Rudo) ( $\mathrm{n}=75)$.

Mean of 3 values (range) interface pressure in $\mathrm{mm} \mathrm{Hg}$, measured using Trickovic sensor placed $8 \mathrm{~cm}$ above medial malleolus with patient in supine position: Group 1: 50 (46-56); Group 2: 44 (37-49). 
Milic 2007 (Continued)

Outcomes
Kaplan-Meier estimate of cumulative proportion of patients with complete healing of study limb at 500 days: Group 1: 93\%; Group 2: 51\%, P $<0.01$.

Kaplan-Meier estimate of median (range) days to healing: Group 1. 133 (28-464); Group 2: 211 (61-438).

Cox regression did not show a relationship between time to healing and any baseline variable.

Recurrence rate during 1-year follow-up: Group 1: 16/67 (24\%); Group 2: 18/34 (53\%), P< 0.05.

Number (\%) patients healed following recurrence, after additional compression therapy using the same regimen: Group 1: 16/16 (100\%); Group 2: 16/18 (89\%).

Number (\%) with adverse events:

Skin excoriation on front of ankle or just below knee: Group 1: 12/72 (17\%); Group 2: not reported.

Slippage of device at knee, causing pressure/pain: Group 1: 34/72 (47\%); Group 2: not reported.

Patients complaining of pain at start of treatment: Group 1: 8/72 (11\%); Group 2: 19/66 (29\%).

\section{Risk of bias}

\begin{tabular}{|c|c|c|}
\hline Bias & Authors' judgement & Support for judgement \\
\hline $\begin{array}{l}\text { Random sequence genera- } \\
\text { tion (selection bias) }\end{array}$ & Low risk & "Randomization was computer generated". \\
\hline $\begin{array}{l}\text { Allocation concealment } \\
\text { (selection bias) }\end{array}$ & Unclear risk & No further details provided. \\
\hline $\begin{array}{l}\text { Incomplete outcome data } \\
\text { (attrition bias) } \\
\text { All outcomes }\end{array}$ & High risk & $\begin{array}{l}\text { Withdrawals: Group } 1: 3 \text { patients ( } 2 \text { lost to follow-up, } 1 \text { had a stroke); Group } \\
\text { 2: } 9 \text { patients ( } 1 \text { died in road-traffic accident, } 8 \text { requested to change treatment } \\
\text { groups). }\end{array}$ \\
\hline $\begin{array}{l}\text { Blinded outcome assess- } \\
\text { ment (healing) }\end{array}$ & Unclear risk & No details given. \\
\hline Baseline comparability & Low risk & Groups appeared reasonably comparable at baseline. \\
\hline
\end{tabular}

Milic 2010

Median participant ages/arm indicate relatively young patients in this trial. Withdrawals: Group 1: 3 patients (lost to follow-up 2, stroke 1); Group 2: 9 patients (died in road-traffic accident 1, requested to change treatment groups 8). Patients reviewed every 2 months during the 1 -year follow-up period.

\begin{tabular}{ll}
\hline Methods & $\begin{array}{l}\text { Single-centred RCT, conducted in Serbia. Randomisation possibly stratified according to baseline calf } \\
\text { circumference and ulcer area, but report not entirely clear (stratified results presented). }\end{array}$ \\
\hline Participants & 131 patients randomised (source population not stated). \\
& Inclusion criteria: patient age $\geq 18$ years; venous leg ulceration confirmed with colour Duplex scan and \\
& ABPI assessment; ulcer surface area $>3 \mathrm{~cm}^{2}$; ulcer duration > 3 months. \\
& Exclusion criteria: ABPI < 0.8; cardiac insufficiency with ejection fraction <35; pregnancy; cancer; dia- \\
betes; unidentified cause of leg ulcer. & \\
& Number of male:female patients: Group 1: 20:22; Group 2: 21:25; Group 3: 19:24. \\
& Median (range) patient age in years: Group 1: 60 (33-76); Group 2: 55 (35-77); Group 3: 57 (32-77).
\end{tabular}


Milic 2010 (Continued)

Median (range) ulcer area in cm²: Group 1: 9 (4-160); Group 2: 9 (3-160); Group 3: 11 (3-150).

Number of patients with ulcer area in $\mathrm{cm}^{2}<5$ vs $5-10$ vs $11-20$ vs $>20 \mathrm{~cm}^{2}$ : Group 1: 12 vs 12 vs 11 vs 7; Group 2: 10 vs 14 vs 14 vs 8; Group 3: 9 vs 12 vs 13 vs 9 .

Median (range) ulcer duration in years: Group 1: 4.5 (0.3-28); Group 2: 3.5 (0.3-42); Group 3: 4.0 (0.3-31).

Number of patients with calf circumference < 33 vs $33-38$ vs $39-43$ vs $>43$ cm: Group $1: 8$ vs 13 vs 13 vs 8 ; Group 2: 8 vs 14 vs 14 vs 10; Group 3: 7 vs 14 vs 13 vs 9.

All patients had had previous episodes of ulceration.

Interventions
Group 1: gauze bandage applied with $50 \%$ overlap; crepe bandage; elastic class III tubular compression with open toes and heels designed to exert graduated compression with $30-40 \mathrm{~mm} \mathrm{Hg}$ at ankle (Tubulcus, Laboratoires Innothera, Arcueil, France). Tubular device available in 5 sizes and fitted according to ankle and calf measurements. If limb dimensions changed during the trial, a tubular device of a different size was applied accordingly ( $n=42$ patients).

Group 2: gauze bandage as above; crepe bandage; tubular compression as above; 1 elastic bandage applied in a spiral configuration with $50 \%$ overlap, $15 \mathrm{~cm}$ wide, $5 \mathrm{~m}$ long, $200 \%$ stretch (Niva, Novi Sad, Serbia) ( $n=46$ patients).

Group 3: gauze bandage as above; crepe bandage; tubular compression as above; 2 elastic bandages as above ( $\mathrm{n}=43$ patients).

All patients: received treatment at a vascular surgery clinic in Nis, Serbia; received mechanical debridement using sterile gauze; dressings changed every 1-7 days depending on exudate; extensive exudate treated with crystal acidum boricum applied topically to wound; dry dressings applied to non-exuding wounds. Patients advised to walk for 30 minutes after bandaging. If limb size remained unchanged, tubular compression was renewed after 6 months; bandages renewed every 3 months; bandages worn day and night. In cases where the original ulcer closed but a new ulcer developed on the same limb, the limb was considered unhealed until the new area had closed. After ulcer healing patients were instructed to continue wearing the tubular elastic stockings.

Outcomes

Number (\%) patients with complete healing at 26 weeks: Group 1: 13/42 (31\%); Group 2: 31/46 (67\%); Group 3: 32/43 (74\%).

Categorical analysis of baseline calf circumference in relation to complete healing:

Number (\%) patients with complete healing at 26 weeks with calf circumference $<33 \mathrm{~cm}$ : Group 1: 6/8 (75\%); Group 2: 5/8 (63\%); Group 3: 1/7 (14\%) (P<0.05 for Group 1 vs Group 3).

Number (\%) patients with complete healing at 26 weeks with calf circumference $33-38 \mathrm{~cm}$ : Group 1: 4/13 (31\%); Group 2: 12/14 (86\%); Group 3: $11 / 14$ (79\%) $(\mathrm{P}<0.05$ for Group 1 vs Group 2, and for Group 1 vs Group 3).

Number (\%) patients with complete healing at 26 weeks with calf circumference $39-43 \mathrm{~cm}$ : Group 1: 2/13 (15\%); Group 2: 11/14 (79\%); Group 3: 12/13 (92\%) (P<0.01 for Group 1 vs Group 2, P < 0.001 for Group 1 vs Group 3).

Number (\%) patients with complete healing at 26 weeks with calf circumference $>43 \mathrm{~cm}$ : Group 1: $1 / 8$ (13\%); Group 2: 3/10 (30\%); Group 3: 8/9 (89\%) (P< 0.01 for Group 1 vs Group 3, P $<0.05$ for Group 2 vs Group 3).

Categorical analysis of baseline ulcer area in relation to complete healing:

Number (\%) patients with complete healing at 26 weeks with ulcer area $<5 \mathrm{~cm}^{2}$ : Group 1: 10/12 (83\%); Group 2: 10/10 (100\%); Group 3: 7/9 (78\%) (no significant differences between groups).

Number (\%) patients with complete healing at 26 weeks with ulcer area 5-10 $\mathrm{cm}^{2}$ : Group 1:3/12 (25\%); Group 2: $9 / 14$ (64\%); Group 3: 9/12 (75\%) (P<0.05 for Group 1 vs Group 2, and for Group 1 vs Group 3). 
Milic 2010 (Continued)

Number (\%) patients with complete healing at 26 weeks with ulcer area 11-20 $\mathrm{cm}^{2}$ : Group 1: 0/11 (0\%); Group 2: 8/14 (57\%); Group 3: 8/13 (62\%) (P< 0.01 for Group 1 vs Group 2, and for Group 1 vs Group 3).

Number (\%) patients with complete healing at 26 weeks with ulcer area $>20 \mathrm{~cm}^{2}$ : Group 1: 0/7 (0\%); Group 2: 4/8 (50\%); Group 3: 8/9 (89\%) (P< 0.01 for Group 1 vs Group 3).

Median (range) time to healing in weeks (all patients): Group 1: 12 (5-24); Group 2: 11 (3-25); Group 3: 14 (5-24) (median test $\mathrm{P}>0.05$ reported by trial authors).

Cox regression suggested larger values of calf circumference and ulcer area to be significant independent predictors of delayed healing $(P=0.01$ and $P<0.001$ respectively). The probability of healing was significantly lower among patients in Groups 1 and 2 when compared with Group $3(P<0.001$ for Group 1 vs Group 3, P = 0.017 for Group 2 vs Group 3).

Number (\%) patients who withdrew from treatment (all because of non-concordance with treatment): Group 1: 1/42 (2\%); Group 2: 1/46 (2\%); Group 3: 9/43 (21\%).

Trial authors reported that there were no deaths or major complications during the study period.

Notes Wound surface area assessed at baseline and every 2 weeks during the trial using digital photography and computerised planimetry.

Ankle sub-bandage pressure measured in supine, sitting and standing positions. Measurements taken at baseline, 4 and 10 weeks, straight after application of compression using a pressure transducer (Kikuhime small probe; MediTrade, Soro, Denmark). Median resting values in supine vs standing positions in mm Hg: Group 1: 36.2 vs 43.9; Group 2: 53.9 vs 68.2; Group 3: 74.0 vs 87.4. Average static stiffness index (difference in pressure between standing and lying positions): Group 1: < 10; Group 2: > 10; Group 3: > 10.

\section{Risk of bias}

\begin{tabular}{|c|c|c|}
\hline Bias & Authors' judgement & Support for judgement \\
\hline $\begin{array}{l}\text { Random sequence genera- } \\
\text { tion (selection bias) }\end{array}$ & Low risk & "Randomization was computer generated ... ". \\
\hline $\begin{array}{l}\text { Allocation concealment } \\
\text { (selection bias) }\end{array}$ & Unclear risk & No details provided. \\
\hline $\begin{array}{l}\text { Incomplete outcome data } \\
\text { (attrition bias) } \\
\text { All outcomes }\end{array}$ & Low risk & $\begin{array}{l}\text { During the treatment period, } 1 \text { patient in Group } 1,1 \text { patient in Group } 2 \text { and } 9 \\
\text { patients in Group } 3 \text { "dropped out of the study due to noncompliance to com- } \\
\text { pression treatment". } \\
\text { Comment: a Kaplan-Meier plot, and tabulated information, in the trial report } \\
\text { indicated that all randomised patients were included in all analyses of healing. }\end{array}$ \\
\hline $\begin{array}{l}\text { Blinded outcome assess- } \\
\text { ment (healing) }\end{array}$ & Unclear risk & $\begin{array}{l}\text { "An open, randomised, prospective, single-centre study was performed ... ". } \\
\text { Comment: it was unclear whether outcome assessment was blinded. }\end{array}$ \\
\hline Baseline comparability & Low risk & Groups appeared comparable at baseline. \\
\hline
\end{tabular}

\section{Moffatt 1999}

\section{Methods}

RCT with allocation by sequential numbers on a randomisation list, stratified by study centre and baseline total ulcerated area on reference limb $\left(\leq \mathrm{or}>10 \mathrm{~cm}^{2}\right)$. Authors estimated that the study had $80 \%$ power, that the $95 \% \mathrm{Cl}$ for the between-group difference in healing rates would not exceed a differ- 


\section{Participants}

Recruited 232 newly-presented patients from community leg ulcer services in the UK (2 study centres). Inclusion criteria: patient $\geq 18$; not pregnant; venous ulceration.

Exclusion criteria: $\mathrm{ABPI}<0.8$; non-venous ulceration; patients who had entered the trial previously. Number of male:female patients: Group 1: 53:62; Group 2: 53:64.

Mean \pm SD patient age in years: Group 1: $67.8 \pm 13.5$; Group 2: $67.1 \pm 15.2$.

Median (range) baseline ulcer duration in weeks: Group 1: 8 (0-2080); Group 2: 7 (0-728).

Proportion of patients with baseline ulcer area < $10 \mathrm{~cm}^{2}$ : Group 1: $82 \%$; Group 2: $84 \%$.

Proportion of patients able to walk freely: Group 1: 74\%; Group 2: 79\%.

Proportions of patients with mobile vs fixed limb: Group 1: $83 \%$ vs $17 \%$ Group 2: $92 \%$ vs $8 \%$

Interventions

All patients: received a low-adherent primary dressing (Tricotex), bandages changed at least weekly.

Group 1: original Charing Cross 4LB comprising wool, crepe, Elset and Coban. Constituents varied slightly according to ankle circumference $(n=115)$.

Group 2: Profore 4LB comprising wool, crepe, Litepress and Co-Plus. Constituents varied slightly according to ankle circumference $(n=117)$.

Following healing, all patients were prescribed compression stockings and returned to regular follow-up clinics.

Outcomes

Number (\%) patients with complete healing at 12 weeks: Group 1: 69/115 (60.0\%); Group 2: 84/117 (71.8\%). Difference $11.8 \%$ (95\% Cl-0.3\%-23.9\%).

Number (\%) patients with complete healing at 24 weeks: Group 1: 84/115 (73\%); Group 2: 89/117 (76\%). Difference 3.0\% (95\% Cl -8.2\%-14.2\%).

Kaplan-Meier estimate of healing at 24 weeks: Group 1: 82\%; Group 2: 84\%.

HR for healing showed a non-significant trend in favour of Group 2: 1.18 (95\% Cl 0.87-1.59), P = 0.28 (stated as adjusted for baseline variables, but unclear exactly which ones).

Quality of life assessed using Nottingham Health Profile at baseline, 12 and 24 weeks (scores 0-100, with lower scores indicating better quality of life). Domains include: energy; bodily pain; emotional reactions; sleep; social isolation; and physical mobility. Mean differences in final scores calculated using ANOVA with adjustment for baseline scores. 208/232 (90\%) patients completed at least 1 follow-up questionnaire (99 in Group 1, 109 in Group 2). There were no statistically significant between-group differences for any domain.

\section{Notes}

In patients with bilateral ulceration, the limb with the larger area of ulceration was studied.

Number (\%) withdrawals: Group 1: 18 (16\%); Group 2: 17 (15\%).

Reasons for withdrawal: Group 1: non-attendance for treatment 9; bandage discomfort 6; treatment changed by other clinician 1; adverse event 2 (exacerbation of arthritis 1; below-knee skin irritation 1).

Group 2: non-attendance for treatment 3; bandage discomfort 9; treatment changed by other clinician 2 ; death 1; adverse event 2 (profuse bleeding from ulcer 1; pressure damage 1).

Adverse events: Group 1: 14 adverse events in total (infection 4, skin irritation 4, excess exudate 2, new ulcer 1, skin irritation and pain 1, other 2); Group 2: 13 adverse events in total (infection 2, skin irritation 3 , pain 1 , skin irritation and pain 2 , skin irritation and new ulcer 1 , infection and pain 1 , other 3 ).

Methods of wound measurement or assessment not stated.

\section{Risk of bias}

Bias Authors' judgement Support for judgement


Moffatt 1999 (Continued)

Random sequence genera- Low risk "Randomisation took place ... by means of sequential numbers on a randomition (selection bias) sation list which was stratified for ulcer size...".

Allocation concealment $\quad$ Unclear risk No further details provided.
(selection bias)

(selection bias)

\section{Incomplete outcome data Low risk} (attrition bias)

All outcomes

Blinded outcome assess- Unclear risk $\quad$ No detail given.
ment (healing)

233 people recruited; 232 had at least 1 follow-up visit; 18 people from Group 1 and 17 from Group 2 withdrew. Analysis by intention to treat.

Baseline comparability Unclear risk $\quad$ Median baseline ulcer duration slightly longer in Group 1.

\title{
Moffatt 2003a
}

Methods RCT (multicentred), with computer-generated randomisation schedules provided to study centres as sequential number lists. Randomisation stratified by study centre and baseline ulcer area ( $\leq$ or $>$ $10 \mathrm{~cm}^{2}$ ). Sample size: original target of 120 patients was not recruited. It was estimated that $54 \mathrm{pa}-$ tients/arm provided $74 \%$ power to detect $25 \%$ difference in healing rates at $5 \%$ significance level.

Participants Recruited 112 newly-presented patients from community leg ulcer clinics in 5 UK study centres. 109 patients comprised the intention-to-treat population (defined as those attending $\geq 1$ follow-up visit).

Inclusion criteria: signs and symptoms of chronic venous ulceration; $A B P I \geq 0.8$; patient age $\geq 18$ years; ankle circumference $>18 \mathrm{~cm}$; baseline ulcer duration $\geq 2$ weeks.

Exclusion criteria: pregnancy; causes of ulceration other than venous disease; active cellulitis treated with systemic antibiotics; previously entered trial.

Number of male:female patients: Group 1: 24:33; Group 2: 23:29.

Mean \pm SD patient age in years: Group 1: $70.2 \pm 14.4$; Group 2: $71.8 \pm 11.3$.

Number of patients with baseline ulcer area $\leq 10 \mathrm{~cm}^{2}$ vs $>10 \mathrm{~cm}^{2}$ : Group 1: 48 vs 9; Group 2: 45 vs 7.

Median (range) baseline ulcer duration in weeks: Group 1: 6 (2-104); Group 2: 6 (2-1040).

Number (\%) patients with previous ulceration: Group 1: 24/57 (42\%); Group 2: 24/52 (46\%).

Number (\%) patients with history of DVT: Group 1: 4/57 (7\%); Group 2: 4/52 (8\%).

Number (\%) patients with diabetes: Group 1: 1/57 (2\%); Group 2: 4/52 (8\%).

Number (\%) patients with rheumatoid arthritis: Group 1: 5/57 (9\%); Group 2: 3/52 (6\%).

Number of patients walking with aid vs walking freely: Group 1: 17 vs 40; Group 2: 7 vs 45.

Number of patients with limb fully mobile vs limited vs fixed: Group 1: 45 vs 12 vs 0; Group 2: 43 vs 7 vs

2.

Number of patients using drugs that could affect healing: Group 1: 1 (steroids); Group 2: 0.

Interventions

\begin{abstract}
All patients: study limb washed using emollient dissolved in tap water, wound debrided and a simple hypoallergenic hydrating cream applied to the surrounding skin. A simple non-adherent dressing was applied to the ulcer, followed by randomised bandage system. Dressings and bandages were changed at least weekly.

Group 1: 4LB (Profore) $(n=57)$;

Group 2: 2-layer bandage (Surepress) $(n=52)$.

All bandages applied according to manufacturers' instructions.

Patients who withdrew from randomised treatment were allocated to an alternative treatment and continued to be followed-up for 24 weeks. After healing, patients were prescribed compression stocking and returned to usual follow-up clinics.
\end{abstract}


Moffatt 2003a (Continued)

Outcomes
Number (\%) patients with complete healing at 12 weeks: Group 1: 40/57 (70\%); Group 2: 30/52 (58\%). Trial authors reported the following measure of effect for this outcome: odds ratio $4.23(95 \% \mathrm{Cl}$ 1.29-13.86), $P=0.02$. Correspondence with trial authors confirmed that this estimate was adjusted for the following baseline variables: sex, ulcer area, ulcer duration, ankle circumference, whether patient taking medication, previous ulceration and limb ABPI.

*Number (\%) patients with complete healing when randomised treatment discontinued: Group 1: 47/57 (82\%); Group 2: 24/52 (46\%). Difference 36\% (95\% Cl 18\%-55\%), $\mathrm{P}<0.001$.

*Number (\%) patients with complete healing at the end of the study period, including withdrawals from randomised treatment, some of whom switched treatment groups: Group 1: 50/57 (88\%); Group 2: 40/52 (77\%) (P value not reported).

Cox regression: $\mathrm{HR}$ for time to healing over 24 weeks 1.18 (95\% $\mathrm{Cl} 0.69-2.02), \mathrm{P}=0.55$ (correspondence with trial authors confirmed that this estimate was adjusted for the following baseline variables: sex, ulcer area, ulcer duration, ankle circumference, whether patient taking medication, previous ulceration and limb ABPI).

Number of adverse events: Group 1: 7 patients, 8 adverse events; Group 2: 19 patients, 21 adverse events. Number of adverse events described as severe: Group 1: 2; Group 2: 2.

Frequency and description of device-related adverse events: Group 1: 6 patients with 7 events (irritation 2, pain/discomfort 1, slippage 1, tissue breakdown 1, excessive pressure 2); Group 2: 17 patients with 27 events (irritation 4, pain/discomfort 7, slippage 9, tissue breakdown 3, excessive pressure 4).

Number (\%) of withdrawals: Group 1: 7/57 (12\%); Group 2: 28/52 (54\%).

Mean days to withdrawal: Group 1: 32; Group 2: 21.

Number (\%) withdrawals with complete healing: Group 1: 3/7 (43\%); Group 2: 16/28 (57\%) (P value not reported but stated between-group difference not statistically significant).

Mean number of dressing changes/week: Group 1: 1.1; Group 2: $1.5(P=0.0002)$.

Mean weekly cost of treatment/patient (based on clinic costs including dressings and other materials, home care costs including nurse time, dressings and other materials, taking into account frequency of dressing changes/week, price year 2000 using average NHS costs): Group 1: GBP 79.91; Group 2: GBP 83.56 .

Mean cost/patient over 24 weeks (based on estimated mean cost per week and assuming $82.5 \%$ rate of wound closure at 24 weeks for both groups, and mean time to healing of 8.2 weeks for both groups): Group 1: GBP 876; Group 2: GBP 916.

Assessment of health-related quality of life (information taken from conference abstract): patients completed SF-36 at baseline, 24 weeks and at healing/withdrawal. Analysis adjusted for baseline scores; number of patients included in analysis not stated. No significant differences between the 2 bandage systems.

Notes

Patients with bilateral ulceration were randomised to 1 treatment only; limb with largest total area of ulceration was studied. Healing defined as full epithelialisation.

Possible imbalance of baseline ulcer duration (range larger in Group 2, median similar for both groups).

*Details of analyses of complete healing were confirmed through correspondence with the author.

The authors surmised that the lower costs in Group 1 were explained by less frequent dressing changes when compared with Group 2.

\section{Risk of bias}


Moffatt 2003a (Continued)

$\begin{array}{ll}\begin{array}{l}\text { Random sequence genera- } \\ \text { tion (selection bias) }\end{array} & \text { Low risk } \\ & \text { sation list that was stratified for ulcer size ... .". }\end{array}$

\begin{tabular}{|c|c|c|}
\hline $\begin{array}{l}\text { Allocation concealment } \\
\text { (selection bias) }\end{array}$ & High risk & $\begin{array}{l}\text { Information from the author suggested that allocation concealment was not } \\
\text { used. }\end{array}$ \\
\hline
\end{tabular}

(selection bias) used.

Incomplete outcome data Low risk
(attrition bias)

112 people were recruited; analysis by intention to treat ("... meant that pa-

All outcomes tients remained in their original randomised groups irrespective of subsequent treatments applied ..."), however only 109 people analysed.

\begin{tabular}{lll}
\hline $\begin{array}{l}\text { Blinded outcome assess- } \\
\text { ment (healing) }\end{array}$ & Unclear risk & No detail provided. \\
\hline Baseline comparability & Unclear risk & $\begin{array}{l}\text { Median ulcer duration similar across groups, although maximum value greater } \\
\text { in group receiving 2-component compression. Impossible to judge for ulcer } \\
\text { area, as neither mean nor median supplied. }\end{array}$ \\
\hline
\end{tabular}

\section{Moffatt 2008}

\section{Methods}

Multi-centred, cross-over RCT. 10 centres overall: 5 in USA, 3 in UK, 2 in Canada. Participants followed for 8 weeks, with crossover at 4 weeks. Sample size estimation based on trial's primary outcome (bandage slippage).

Participants

Recruited 81 participants with venous leg ulcers from free-standing wound clinics or wound clinics associated with community hospitals or trusts.

Inclusion criteria: patients aged $\geq 18$ years ( 21 years in USA) with 1 or more venous leg ulcers treated with compression for at least 2 weeks prior to study enrolment; able to understand and answer questionnaire items.

Exclusion criteria: patients unsuitable for compression therapy or study enrolment; $A B P I<0.8$ within 4 weeks of start of trial; circumferential leg ulcer; ulcer with signs of clinical infection.

Number of male:female patients: Group 1: 25:14; Group 2: 22:20.

Mean \pm SD patient age in years: Group 1: $62.5 \pm 15.5$; Group 2: $63.5 \pm 12.5$.

Mean \pm SD ulcer area in $\mathrm{cm}^{2}$ : Group 1: $11.8 \pm 19.7$; Group 2: $5.7 \pm 7.9$.

Mean \pm SD ulcer duration in weeks: Group 1: $186.3 \pm 438.7$; Group 2: $195.1 \pm 512.1$.

Number of patients walking with vs without assistance: Group 1: 7 vs 32; Group 2: 6 vs 36.

Mean \pm SD health-related quality of life scores (overall): Group 1: $6.0 \pm 2.0$; Group 2: 7.1 \pm 2.1 .

Interventions
Group 1: 2-component compression bandage system consisting of 2 latex-free roll bandages (3MTM CobanTM 2 Layer Compression System; 3MTM Health Care, St Paul, MN, USA). Inner component of polyurethane foam laminated to a cohesive bandage; outer layer is a cohesive bandage $(n=39$ patients).

Group 2: 4LB (ProforeTM Multi-Layer Bandaging System; Smith \& Nephew Medical Wound Management, Hull, UK) ( $n=42$ patients).

All patients: apart from the compression therapy, patients received standard care as provided by each centre. All ulcers were covered with a foam dressing (Tegaderm TM Foam Dressing; 3MTM Health Care). Other wound treatments such as antimicrobial dressings were applied underneath the foam dressing. Bandages changed weekly, or more frequently, as required. Medications and additional wound treatments permitted at the discretion of the study investigator.Tubular support systems underneath the 

were not allowed within 2 weeks prior to enrolment, or at any time during the trial.

Outcomes

Number (\%) patients with complete healing at 4 weeks: Group 1: 6/39 (15\%); Group 2: 3/42 (7\%).

Median (range) \% change in wound surface area at 4 weeks: Group 1: -27.8 (-100-233.3); Group 2: -42.2

$(-100-272.1), P=0.88$, Wilcoxon rank sum test.

Median (range) linear healing rate in cm/week : Group 1: 0.04 (-0.16-0.40); Group 2: 0.04 (-0.27-0.19), $\mathrm{P}=$ 0.94 , Wilcoxon rank sum test.

Health-related quality of life assessed using the Cardiff Wound Impact Schedule for 3 domains (wellbeing; physical symptoms and daily living; social life) as well as overall health-related quality of life and the patient's satisfaction with their overall health-related quality of life. Significant difference in favour of the 2-component system during the pre-crossover period for physical symptoms and daily living scores $(P<0.05$, 2-sample pooled t-test, per protocol analysis). There were no other significant differences in either the pre- or post-crossover period.

Adverse events: overall, 41 patients reported at least 1 adverse event. Of 135 adverse events, 67 occurred during use of the 4LB and 68 during use of the 2-component system. 92 adverse events deemed to be unrelated to compression and 43 as possibly, or probably, related to compression. 2 patients hospitalised during the trial for reasons unrelated to compression (intestinal bleeding and renal failure); these 2 patients had multiple adverse events accounting for 45/135 reported adverse events.

Patient withdrawals during 1st 4-week period: Group 1: 2 (both due to adverse events); Group 2: 1 (patient request).

Patient withdrawals during 2nd 4-week period: Group 1 (received 2-component compression first, then 4LB): 3 (lack of concordance 1, investigator decision 1, adverse event 1); Group 2 (received 4LB first then two-component compression): no withdrawals.

Notes

Patients with bilateral leg ulcers received the same compression system on both legs. For patients with more than 1 ulcer, investigators used clinical judgement to choose which ulcer or leg to follow in the study prior to randomisation. Exact criteria for choice of ulcer or leg not explained.

Primary outcome of trial was bandage slippage measured at each bandage change (significant difference in favour of 2-component system, mean slippage 2.48 vs $4.17 \mathrm{~cm}, \mathrm{P}<0.001$ ). Secondary outcomes were wound healing (assessed with tracings and computerised planimetry), health-related quality of life, bandage wear time (no significant difference between groups), patient mobility (available data were limited but suggested no significant difference between groups), and patient preference (72\% preferred 2-component system, $22 \%$ preferred 4 LB, $6 \%$ had no preference).

Compression bandages applied by a trained study co-ordinator under supervision of the study investigator for each centre. Investigators selected on the basis of prior knowledge and experience of using the 4LB. 4LB applied according to manufacturer's instructions and additional training deemed unnecessary. All staff and investigators were provided with training in applying the 2-component system by manufacturer's regional technical expert; staff had to demonstrate proficiency in bandaging technique prior to start of enrolment.

\section{Risk of bias}

Bias Authors' judgement Support for judgement

Random sequence genera- Low risk tion (selection bias)
"The randomisation was stratified by study site so that the treatment order assignment was kept balanced within each site. The randomisation schedule was computer generated by the study biostatistician and provided to the investigators in sealed envelopes and opened only after subject enrolment and selection of the study leg/wound".

\begin{tabular}{|c|c|c|}
\hline $\begin{array}{l}\text { Allocation concealment } \\
\text { (selection bias) }\end{array}$ & Unclear risk & $\begin{array}{l}\text { "The randomisation schedule was computer generated by the study biostatis- } \\
\text { tician and provided to the investigators in sealed envelopes and opened only } \\
\text { after subject enrolment and selection of the study leg/wound". }\end{array}$ \\
\hline
\end{tabular}

Compression for venous leg ulcers (Review)

Copyright $\odot 2013$ The Cochrane Collaboration. Published by John Wiley \& Sons, Ltd. 

numbered and opaque.

Incomplete outcome data Low risk
(attrition bias)

All outcomes

\begin{abstract}
"Unless otherwise stated, data from all enrolled subjects were analysed on an intent-to-treat (ITT) basis".

"Wound healing ... Of the 79 wounds entered into the analysis ... ".

Comment: $79 / 81$ (98\%) patients were included in the analysis of healing. From the flow diagram provided, we assumed that the 2 excluded patients were those noted as errors in randomisation (1/treatment group).
\end{abstract}

$\begin{array}{ll}\begin{array}{l}\text { Blinded outcome assess- } \\ \text { ment (healing) }\end{array} & \text { "Subject or investigator blinding was not possible because of the obvious dif- } \\ \text { ferences between the two-layer and four-layer systems; however, the individ- } \\ \text { ual conducting the wound-tracing measurements was blinded to treatment". }\end{array}$

Regarding "baseline patient and wound characteristics ... there were no significant differences in any of the measured parameters, indicating that the two treatment groups were similar in make up".

Comment: baseline ulcer area appeared smaller in Group 2, however, it was difficult to judge with confidence, as means, rather than medians, were presented.

Moody 1999

Methods RCT (method of allocation not stated beyond 'randomised'). Study conducted in the UK, other details of setting not reported.
Participants
52 patients recruited
Inclusion criteria: patient age $\geq 18$ years; mobile; venous leg ulcer $>2 \mathrm{~cm}$ at widest perpendicular diame- ter; $\mathrm{ABPI} \geq 0.8$.
Number of male:female patients: Group 1: 7:19 Group 2: 7:19.
Average (range) patient age in years: Group 1: 73 (51-85); Group 2: 70 (45-88).
Mean baseline ulcer duration in months: Group 1: 55; Group 2: 46 (no variance data presented).

Interventions

Where possible, patients had study limb immersed in warm water with added emollient, then dried. Ulcer was irrigated with a saline spray and a primary dressing applied (Solvaline $\mathrm{N}$ for wounds with little exudate and Silicone NA Ultra for moderate to high levels of exudate). Dressings and bandages changed according to need, taking in to account exudate, bandage slippage and patient preference. Dressings/bandages re-applied either at clinic or at patient's home.

Group 1: under cast padding (Cellona) plus SSB (Rosidal K) $(n=26)$.

Group 2: under cast padding (SurePress padding) plus long-stretch compression bandage (SurePress bandage) $(\mathrm{n}=26)$.

Both bandages applied using a simple spiral technique.

Outcomes

Outcomes assessed at 12 weeks. Patients seen weekly by a research nurse. Wounds photographed at regular intervals.

Number (\%) patients with complete healing at 12 weeks: Group 1: 8/26 (31\%); Group 2: 8/26 (31\%).

Average (presumably mean, but not stated) weeks to healing: Group 1: 9.91; Group 2: 9.3 (no variance data presented). 
Moody 1999 (Continued)

Average (presumably mean, but not stated) percentage reduction in ulcer area at 12 weeks (measured by a single assessor using computerised analysis of weight of cut-out acetate tracing of wound perimeter): Group 1: 73\%; Group 2: 52\% (no variance data presented).

Number (\%) patients with increase in ulcer size during study: Group 1: 4/26 (15\%); Group 2: 6/26 (23\%).

Number (\%) patients with clinical infection developing during study period: Group 1: 3/26 (12\%); Group 2: $4 / 26(15 \%)$.

Notes 1 ulcer/patient included in the study.

Changes in sub-bandage pressure assessed over a 7-day period by means of an Oxford pressure monitor. These measurements appear to have been performed on healthy volunteers.

Training in application of both types of bandages offered to study care providers. Bandages applied according to manufacturers' instructions. Authors reported that, by the end of the study, around 7 patients/group (or their relatives) could apply the bandages correctly.

1 patient had an acute eczema episode during the study and 1 had a chest infection (group allocation not stated).

3 patients in Group 1 experienced initial bandage slippage due to reduction of limb oedema, necessitating re-application of the bandage within $6 \mathrm{~h} .1$ patient withdrew because of difficulties in performing bandage re-application with adequate frequency.

No information provided on baseline ulcer area. On average, patients in Group 1 had ulcers of longer duration at baseline.

Few details were provided on data analysis methods.

Changes in limb oedema were reported in the paper.

Unable to gain further information from trial author.

\section{Risk of bias}

\begin{tabular}{|c|c|c|}
\hline Bias & Authors' judgement & Support for judgement \\
\hline $\begin{array}{l}\text { Random sequence genera- } \\
\text { tion (selection bias) }\end{array}$ & Unclear risk & No details provided beyond describing the trial as "randomised". \\
\hline $\begin{array}{l}\text { Allocation concealment } \\
\text { (selection bias) }\end{array}$ & Unclear risk & No details provided. \\
\hline $\begin{array}{l}\text { Incomplete outcome data } \\
\text { (attrition bias) } \\
\text { All outcomes }\end{array}$ & Unclear risk & $\begin{array}{l}\text { Report stated the number of people healed in each group, but denominator at } \\
\text { end of follow-up unclear. }\end{array}$ \\
\hline $\begin{array}{l}\text { Blinded outcome assess- } \\
\text { ment (healing) }\end{array}$ & Unclear risk & No details provided. \\
\hline Baseline comparability & Unclear risk & $\begin{array}{l}\text { Little information. Mean ulcer duration appeared to be longer in Group } 1 \text {, but } \\
\text { no variance data presented or data on other variables. }\end{array}$ \\
\hline
\end{tabular}

Morrell 1998

$\begin{array}{ll}\text { Methods } & \text { RCT (multicentred, pragmatic, i.e. reflecting everyday clinical practice as far as possible). Patients allo- } \\ \text { cated to treatment groups according to a random assignment schedule prepared in advance of recruit- } \\ \text { ment. Randomisation was separate for each study site. Outcome assessment was non-blind. Sample }\end{array}$


Morrell 1998 (Continued)

size: estimated that 206 patients were required to provide $80 \%$ power to detect an increase in healing from $50 \%-70 \%$, at $5 \%$ significance level.

\section{Participants}

233 patients recruited from 8 community-based research clinics in 4 health trusts in Trent, UK. Inclusion criteria: venous leg ulcer of at least 3 months' duration at study entry; ability to travel to clinic.

Exclusion criteria: $\mathrm{ABPI}<0.8$.

Number of male:female patients: Group 1: 43:77; Group 2: 35:78.

Mean \pm SD patient age in years: Group 1: $73.8 \pm 10.9$; Group 2: $73.2 \pm 11.6$.

Mean \pm SD baseline ulcer surface area in $\mathrm{cm}^{2}$ : Group 1: $16.2 \pm 28.9$; Group 2: $16.9 \pm 40.8$.

Mean \pm SD baseline ulcer duration in months: Group 1: $27.5 \pm 53.8$; Group 2: $29.7 \pm 82.3$.

Mean \pm SD body mass index $\left(\mathrm{kg} / \mathrm{m}^{2}\right)$ : Group 1: $27.0 \pm 6.7$; Group 2: $27.1 \pm 6.0$.

Number (\%) patients requiring aid with walking: Group 1: 66/120 (55\%); Group 2: 57/113 (50\%).

Number (\%) patients with history of DVT: Group 1: 28/120 (23\%); Group 2: 25/113 (22\%).

Number (\%) patients with diabetes mellitus: Group 1: 8/120 (7\%); Group 2: 10/113 (9\%).

Patients were assessed for health status at baseline using SF-36, EuroQol, the McGill short form pain questionnaire and the Frenchay activities index. Groups were comparable at baseline for all domains.

Interventions

Group 1: weekly treatment with 4LB in a leg ulcer clinic. The Charing Cross technique was used, comprising non-adherent primary dressing, absorbent orthopaedic wadding, crepe bandage, elastic compression bandage, cohesive compression bandage. Clinic co-ordinators all completed course on leg ulcer management (ENB N18), and additional training in application of 4LBs. Each clinic employed support nurses trained in the application of 4 LB. After healing, patients received class II compression stockings and were reviewed at the clinic every 3 months. Transport was provided free of charge to patients $(n=120)$.

Group 2: usual care at home by district nursing service. Frequency of visits varied and could be sever$\mathrm{al} /$ week. A variety of wound cleansers, primary dressings, topical agents, securing agents and bandages were used. The bandages included compression, Tubigrip and light support bandages, all of which could be used alone or with other devices. Access to 4 LBs was minimal $(n=113)$.

Outcomes Number (\%) patients with complete healing at 12 months: Group 1: 78/120 (65\%); Group 2: 62/113 (55\%).

Kaplan-Meier estimates of cumulative \% healed at 12 weeks: Group 1: 34\%; Group 2: 24\% (difference $10 \%, 95 \% \mathrm{Cl}-2 \%-22 \%)$.

Kaplan-Meier estimate of median weeks to healing within 12 month follow-up period: Group 1: 20; Group 2: 43 ( $P=0.03$, log rank test).

Cox regression: following adjustment for prognostic factors (patient age, baseline ulcer area, baseline ulcer duration, history of DVT) estimated hazard ratio was 1.65 (95\% Cl 1.15-2.35, P value not reported) (in favour of Group 1).

Number (\%) patients with recurrence following initial healing during trial: Group 1: 27/78 (35\%); Group 2: $14 / 62(23 \%)$.

The between-group difference in time to recurrence was not statistically significant $(P=0.38$, log rank test).

Mean ulcer-free weeks during 12 month follow-up: Group 1: 20.1; Group 2: 14.2 (difference 5.9, 95\% Cl 1.2-10.5).

No significant differences found between the groups in change in health status.

Mean \pm SD total NHS costs/patient/year (baseline analysis, GBP, price year 1995): Group 1: GBP $877.60 \pm$ 674.30; Group 2: GBP 863.09 $\pm 865.32(P=0.90)$. Baseline analysis based on cost of treatment (staff time, materials, transport, overheads) and cost of other health services (GP and hospital). Sensitivity analyses assessed effects of changing treatment costs and overheads in Group 2, and changes in clinic attendance costs in Group 1. Authors reported that changes in assumptions did not significantly alter the 
Morrell 1998 (Continued)

magnitude of estimated costs (central estimates shown, no data on variance or statistical tests of between-group differences).

\section{Notes}

Withdrawals: Group 1: 17 (died 9, moved away 2, hospital admission 3, dropped out with no further information available 3); Group 2: 23 (died 7, referred elsewhere 3, moved away 6, hospital admission 3, nursing home admission 3 , dropped out with no further information available 1 ).

Complete healing defined as re-epithelialisation of all patient's areas of ulceration. Wound surface area measured every 4 weeks using tracing from photographs combined with computerised planimetry. Fine indelible pens were used to obtain tracings. Assessors were trained in an attempt to standardise measurement techniques and minimise inter-rater error.

\section{Risk of bias}

\begin{tabular}{|c|c|c|}
\hline Bias & Authors' judgement & Support for judgement \\
\hline $\begin{array}{l}\text { Random sequence genera- } \\
\text { tion (selection bias) }\end{array}$ & Low risk & $\begin{array}{l}\text { "A random assignment schedule and serially numbered, sealed, opaque allo- } \\
\text { cation envelopes were prepared in advance for each of the } 8 \text { clinic sites". }\end{array}$ \\
\hline $\begin{array}{l}\text { Allocation concealment } \\
\text { (selection bias) }\end{array}$ & Low risk & $\begin{array}{l}\text { "Serially numbered, sealed opaque allocation envelopes were prepared in ad- } \\
\text { vance for each of the } 8 \text { study sites". }\end{array}$ \\
\hline $\begin{array}{l}\text { Incomplete outcome data } \\
\text { (attrition bias) } \\
\text { All outcomes }\end{array}$ & Low risk & "All the data analysis was by intention to treat". Survival analysis. \\
\hline $\begin{array}{l}\text { Blinded outcome assess- } \\
\text { ment (healing) }\end{array}$ & High risk & $\begin{array}{l}\text { "The nurse recorded the date of healing, defined as the data of epithelialisa- } \\
\text { tion of all ulcers...". }\end{array}$ \\
\hline Baseline comparability & Unclear risk & $\begin{array}{l}\text { Only means presented; these appear similar, but data likely to be highly } \\
\text { skewed. }\end{array}$ \\
\hline
\end{tabular}

Nelson 2007a

Methods

RCT with $2 \times 2 \times 2$ factorial design evaluating: drugs - pentoxifylline vs placebo; dressings - knitted viscose vs hydrocolloid; and compression bandages - 4LB vs single-layer. Randomisation stratified by study centre and ulcer type (simple venous vs non-simple) using permuted blocks of 8 . Outcome assessment was non-blind. Sample size: assuming $40 \%$ healing rate at 24 weeks using 4 LB or knitted viscose dressing, it was estimated that 200 patients would provide $80 \%$ power to detect $20 \%$ difference in healing rates at 24 weeks at $5 \%$ significance level (2-tailed).

Participants

Recruited 245 patients with venous leg ulcers treated in community or as outpatients from 2 centres in Falkirk and Edinburgh (UK). All study centres had widespread use of high compression prior to the trial. Inclusion criteria: patient age > 18 years; clinical signs of venous disease; venous disease confirmed with hand-held Doppler; venous leg ulcer $\geq 1 \mathrm{~cm}$ length and $\geq 8$ weeks' duration.

Exclusion criteria: severe concurrent disease; life expectancy < 6 months; immunosuppressed; immobile; $\mathrm{ABPI}<0.8$; diabetes mellitus; taking warfarin, steroids, pentoxifylline, oxerutins or naftidrofuryl; infected or gangrenous ulcers; pregnancy, lactating or premenopausal not using contraception; sensitivity to methylxanthines or caffeine.

Number of male:female patients: Group 1: 39:89; Group 2: 41:76.

Mean \pm SD, median (range) patient age in years: Group 1: $71.5 \pm 10.3$, 73 (46-93); Group 2: $68.3 \pm 12.2,68$ (34-91).

Mean \pm SD, median (range) baseline ulcer area in mm2: Group 1: $1025 \pm 2637$, 385 (54-26,311); Group 2: $661 \pm 879,393$ (50-5560).

Mean \pm SD, median (range) baseline ulcer duration in months: Group 1: $11.1 \pm 17.3$, 5.0 (2-96); Group 2: $15.1 \pm 35.2,5.0$ (2-240).

Number (\%) patients walking without aid: Group 1: 49/128 (38\%); Group 2: 36/117 (31\%). 
Number (\%) patients with simple vs non-simple venous disease (non-simple defined as seropositive rheumatoid arthritis or venous pathology not confirmed with hand-held Doppler): Group 1: 103 (80\%) vs 25 (20\%); Group 2: 97 (83\%) vs $20(17 \%)$.

Number (\%) patients randomised to pentoxifylline vs placebo: Group 1: 64/128 (50\%) vs 64/128 (50\%); Group 2: 57/117 (49\%) vs 60/117 (51\%).

Number (\%) patients randomised to knitted viscose dressing vs hydrocolloid dressing: Group 1: 62/128 (48\%) vs 66/128 (52\%); Group 2: 65/117 (56\%) vs 52/117 (44\%).
All patients: ulcers cleansed with tap water and surrounding skin moisturised with arachis or olive oil. Dressings and bandages renewed at least weekly.

Group 1: single-layer bandage (hydrocolloid-lined, woven, elastomeric, adhesive bandage applied in a figure-of-8 technique from toe to knee) $(n=128)$.

Group 2: 4LB, Charing Cross technique comprising wool, crepe, Elset, Coban ( $n=117)$.

Also randomised comparison of dressings (knitted viscose dressing or hydrocolloid) and drug treatment (pentoxifylline or placebo).

\section{Analyses based on 245 patients with simple and non-simple venous ulceration:}

Number (\%) patients with complete healing at 24 weeks: Group 1:63/128 (49\%); Group 2: 78/117 (67\%), $\mathrm{P}=0.009$.

Median days to healing (Kaplan-Meier estimate): Group 1: 168; Group 2: 78 (P value not reported).

Cox proportional hazards models: an initial model including terms for drug, dressing and bandage and all possible interactions (but no terms for baseline characteristics) did not detect any statistically significant interaction between the different treatments $(P>0.14)$; a subsequent model adjusted for drug, dressing, bandage, study centre, ulcer aetiology (simple or non-simple), baseline ulcer area, baseline ulcer duration, and history of ulceration (years since first ulcer), $\mathrm{HR} 2.0(95 \% \mathrm{Cl} 1.4-2.9), \mathrm{P}<0.0005$, in favour of Group 2. The following were significant independent predictors drug $(P=0.046)$, baseline area $(P<0.0005)$, ulcer duration $(P=0.017)$ and ulcer history $(P=0.01)$.

Withdrawals (bandages and dressings considered together): overall, 68/245 (28\%) withdrew from original bandage or dressing or both. Number (\%) patients changed bandage due to adverse event: Group 1: 36/128 (28\%); Group 2: 17/117 (15\%). Estimates from logistic regression indicated a statistically significant interaction between dressing and bandage in terms of predicting withdrawal $(P<0.001)$.

\section{Analyses based on 200 patients with simple venous ulceration:}

Number (\%) patients with complete healing at 24 weeks: Group 1: 50/103 (49\%); Group 2: 67/97 (69\%).

Quality of life assessment: assessed with Nottingham Health Profile at baseline and 24 weeks (scores 0-100 with lower scores indicating better quality of life). Domains: energy; pain; emotional reactions; sleep; social isolation; and physical mobility. Mean between-group differences in final scores adjusted for baseline scores; analysis was by intention-to-treat. Patients in Group 2 ( $n=95$ available) had significantly greater improvement (adjusted mean difference, $95 \% \mathrm{Cl}$ ) in the following when compared with Group 1 ( $n=98$ available): energy $7.9(0.2-15.6), P=0.04$; and physical mobility $4.5(0.0-9.0), P=$ 0.046 . Mean differences for the other domains were not statistically significant between the 2 bandage groups.

Withdrawals: overall 65/200 (32.5\%). Number (\%) patients who withdrew first from bandage system with or without simultaneous withdrawal from the randomised drug and dressing treatment: Group 1: 21/103 (20\%); Group 2: 5/97 (5\%).

Notes

Treatment for all patients delivered by experienced leg ulcer nurses.

Healing defined as complete epithelial cover in the absence of scab for all ulcers on study limb.

Ulcer area measured by transparency tracing and blind scanning.

\section{Risk of bias}

Bias Authors' judgement Support for judgement


Nelson 2007a (Continued)

Random sequence genera- Unclear risk No details given.
tion (selection bias)

$\begin{array}{ll}\begin{array}{l}\text { Allocation concealment } \\ \text { (selection bias) }\end{array} & \text { "Sow risk } \\ & \begin{array}{l}\text { ticipants to placebo or pentoxifylline, knitted viscose or hydrocolloid dress- } \\ \text { ings, and four-layer or adhesive single-layer bandages". }\end{array}\end{array}$

\begin{tabular}{lll}
\hline $\begin{array}{l}\text { Incomplete outcome data } \\
\text { (attrition bias) } \\
\text { All outcomes }\end{array}$ & Low risk & "Primary analysis was by intention to treat". Survival analysis. \\
\hline $\begin{array}{l}\text { Blinded outcome assess- } \\
\text { ment (healing) }\end{array}$ & High risk & $\begin{array}{l}\text { "Nurses completed a dressing log at each leg ulcer dressing visit which record- } \\
\text { ed whether or not an ulcer was healed". }\end{array}$ \\
\hline $\begin{array}{ll}\text { Baseline comparability } & \text { Low risk }\end{array}$ & $\begin{array}{l}\text { Medians provided for ulcer area and duration that appear fairly well balanced, } \\
\text { plus analysis was adjusted (Cox regression). }\end{array}$ \\
\hline
\end{tabular}

\section{O'Brien 2003}

Methods RCT (pragmatic, i.e. reflecting everyday clinical practice as far as possible). Randomisation achieved by computer-generated list. Estimated a priori that the study had $80 \%$ power of detecting a $20 \%$ between-group difference in healing rates at 12 weeks, at $5 \%$ significance level. Trialists also considered the sample size appropriate to detect differences in quality of life (but statistics for this not provided).

\begin{tabular}{|c|c|}
\hline Participants & 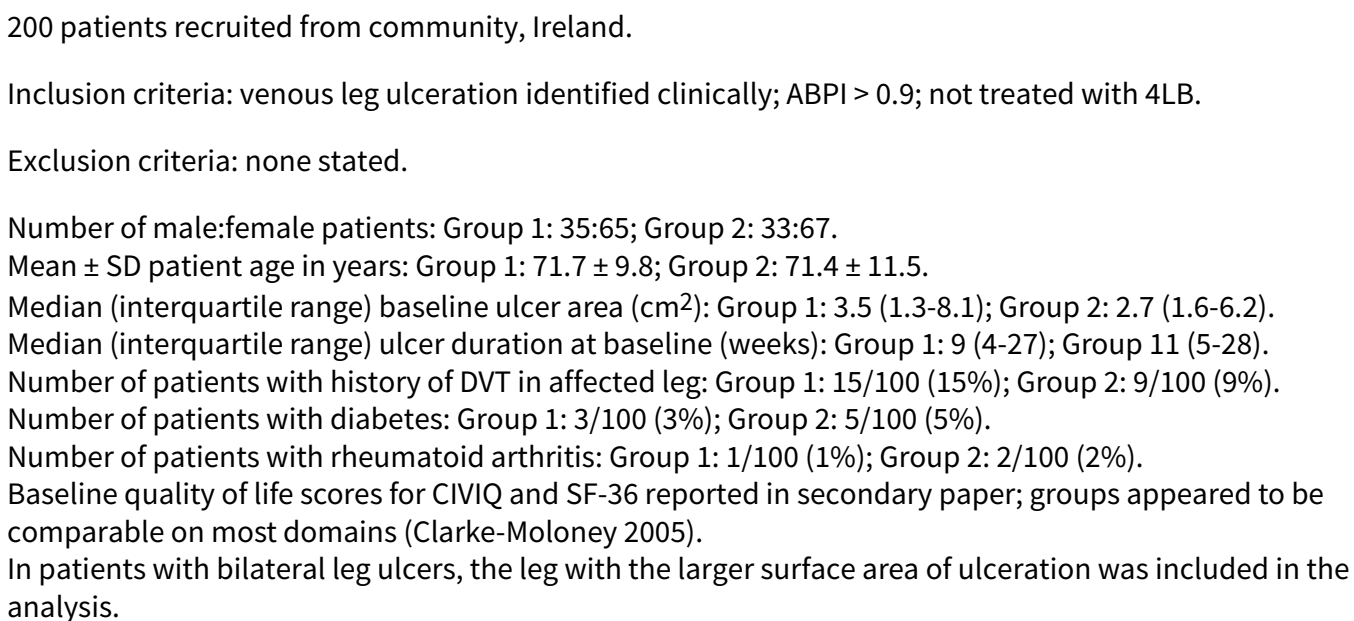 \\
\hline
\end{tabular}

Interventions All treatments provided in a community setting.

Group 1: 4LB application standardised and comprised: sterile wound contact layer, padding bandage, light conformable bandage, light compression bandage, and flexible cohesive bandage. Combined system provided compression of $40 \mathrm{mmHg}$ at the ankle (measurement method not explained). 12 patients non-compliant due to intolerance of bandage. 11 patients had high absorbency dressings and 8 patients had desloughing agents $(n=100)$.

Group 2: usual care - treatment not standardised, but determined by public health nurse or GP. Treatment included assortment of topical applications such as hydrocolloids, alginates, paraffin and iodine dressings; dressings of various absorbencies; and low-pressure bandages and elasticated support. 1 patient had laser therapy; 5 patients had compression at some stage during the trial $(n=100)$.

\section{Outcomes}

All patients were followed-up for 12 weeks. 
Proportions healed at 12 weeks (from Kaplan-Meier analysis): Group 1: 54\%; Group 2: $34 \%(P<0.001)$.

Time to healing significantly better in Group $1(P=0.006$, log rank test).

Healing rates remained significantly different after controlling for age, baseline ulcer area, baseline ulcer duration, DVT, diabetes and rheumatoid arthritis in Cox regression $(P=0.015)$.

The mean difference $(95 \% \mathrm{Cl})$ in reduction in ulcer size between the 2 groups was not significantly different: $-1.1(-2.9-0.7)$.

Costs/leg healed based on dressing use, nursing time (for dressings, administration and travel) and nurses' mileage expenses. Median (interquartile range) overall cost/leg healed in EUR (presume price year same as trial accrual period, i.e. 1999-2000): Group 1: EUR 209.7 (137.5-269.4); Group 2: EUR 234.6 (168.2-345.1), $\mathrm{P}=0.04$.

Health-related quality of life assessed during treatment (at 6 weeks) in unhealed patients: Group 1: 79/85 (93\%); Group 2: 91/95 (96\%). Overall, Group 1 achieved better quality of life benefits compared with Group 2, particularly in areas of physical activity and social functioning.

Disease specific instrument (CIVIQ - 20 items covering 4 domains: psychosocial, physical functioning, social functioning, and pain; lower scores reflect better quality of life): between-group difference at 6 weeks significant for physical functioning $(P=0.006)$, social functioning $(P=0.001)$ and global score $(P=$ 0.006), all differences in favour of Group 1. Full statistics on scores in paper (Clarke-Moloney 2005).

Generic instrument (SF-36: 36 items covering 8 domains: physical functioning, role limitation due to physical health, bodily pain, general health, vitality, social functioning, role limitation due to emotional problems, and mental health; higher scores reflect better quality of life). Between-group difference at 6 weeks was significant for physical functioning $(P=0.001)$, role limitation - physical $(P=0.006)$ and mental health $(P=0.03)$, all differences in favour of Group 1. Full statistics on scores in paper (ClarkeMoloney 2005).

Notes

Stated that ulcerated area measured and photographed by a research officer, but wound measurement instrument not described.

All leg ulcer dressings done by usual community nurse. Before study started, all public health nurses in the region given formal training (workshops and individual instruction) in application of 4LB.

Patient follow-up during trial: Group 1: 1 died, 2 lost to follow-up; 98 full or partial data gathered; Group 2: 0 died, 0 lost to follow-up, 100 full or partial data gathered.

\section{Risk of bias}

\begin{tabular}{lll}
\hline Bias & Authors' judgement & Support for judgement \\
\hline $\begin{array}{l}\text { Random sequence genera- } \\
\text { tion (selection bias) }\end{array}$ & Low risk & $\begin{array}{l}\text { "A random intervention and control list was generated for } 200 \text { patients by } \\
\text { computer ...". }\end{array}$ \\
\hline $\begin{array}{l}\text { Allocation concealment } \\
\text { (selection bias) }\end{array}$ & Low risk & $\begin{array}{l}\text { "Before the study began, a random 'intervention' or 'control' list was generat- } \\
\text { ed for } 200 \text { patients by computer, and the results were entered sequentially in- } \\
\text { to sealed numbered envelopes. These envelopes were assigned to consecutive } \\
\text { patients once consent had been obtained". }\end{array}$
\end{tabular}

Incomplete outcome data Low risk

"Intention to treat analysis was carried out".

(attrition bias)

All outcomes 
O'Brien 2003 (Continued)

Blinded outcome assessment (healing)
Unclear risk
"When complete healing occurred in the 12 week interval, a photograph of the site was taken to provide an objective review of outcome ... ". It is not clear if assessment of photographs was masked.

Baseline comparability High risk Median ulcer area larger in Group 1.

\section{Partsch 2001}

Methods

RCT (multicentred) with stratification by study centre and total ulcerated area of study limb ( $\leq$ or $>10$ $\mathrm{cm}^{2}$ ). Sample size: estimated 112 patients would provide $77 \%$ power to detect a $25 \%$ difference in proportion of patients healed at 16 weeks at $5 \%$ significance level (2-sided test).

\section{Participants}

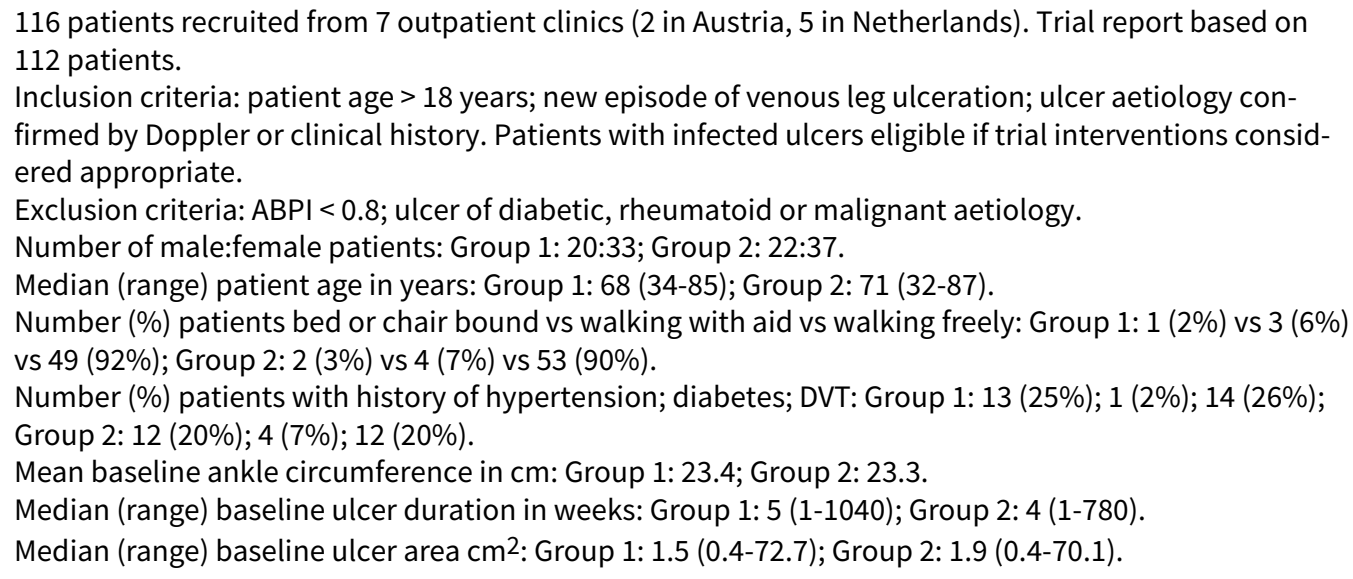

Interventions

All patients: ulcers cleansed with water or saline and covered with simple non-adherent dressing. Ulcers in the hollow behind the malleolus also covered with a foam pad to increase local pressure. Bandaging weekly unless more frequent dressing changes required (median interval between visits $=7$ days for both groups). Patients encouraged to walk as much as possible.

Group 1: 4LB (Profore) $(n=53)$.

Group 2: SSB comprising orthopaedic padding plus 2 SSBs (Rosidal K) applied using the Putter technique $(n=59)$. portion healed $11 \%(95 \% \mathrm{Cl}-28-7 \%)$.

Kaplan-Meier estimates: cumulative proportions healed at 16 weeks Group 1: 78\% Group 2: 85\%; median $(95 \% \mathrm{Cl})$ days to healing Group 1: 57 (47-85) Group 2: 63 (43-70).

Cox regression: an initial model containing terms for treatment and study centre showed a centre effect, with $4 / 7$ centres having a higher healing rate than the other $3(P=0.003)$. No evidence of a centre effect when models were re-fitted including terms for initial area and duration of ulcer $(P=0.79)$. Final model included terms for treatment, study centre, baseline ulcer area, baseline ulcer duration and the SF-36 dimension 'mental health. HR 1.19 (95\% Cl 0.73-1.91), P = 0.49 (represents non-significant trend towards higher healing rate for Group 2).

Withdrawals for patients not included in analysis, breakdown/group not reported: 3 patients had no post-treatment follow-up data; 1 patient had basal cell carcinoma.

Number of withdrawals during trial for patients included in analysis: Group 1: 12 (patient's request 7, lost to follow-up 3, adverse event 1, other 1); Group 2: 7 (patient's request 2, lost to follow-up 2, lack of response 1 , adverse event 1 , other 1 ). Further details of adverse events not reported. 
Partsch 2001 (Continued)

Notes
Patients with bilateral ulceration randomised to 1 treatment only, limb studied had the larger total area of ulceration. Ulcers measured using tracing and computerised planimetry.

Stated that whilst staff at all participating centres were trained in the application of 4LB prior to the study, they all had many years of experience of applying the SSB.

\section{Risk of bias}

\section{Bias}

Random sequence genera- Low risk tion (selection bias)

\section{Authors' judgement Support for judgement}

Information from published trial report was unclear:

"Randomisation was carried out separately for each centre and further stratified according to whether the total reference limb ulcerated area was less than or equal to $10 \mathrm{~cm}^{2}$, or greater than $10 \mathrm{~cm}^{2}$." No further detail given, however, standard data checks undertaken for the IPD meta-analysis suggested that the random sequence generation was satisfactory.

\begin{tabular}{|c|c|c|}
\hline $\begin{array}{l}\text { Allocation concealment } \\
\text { (selection bias) }\end{array}$ & Low risk & $\begin{array}{l}\text { From published trial report: "Randomisation was carried out separately for } \\
\text { each centre and further stratified according to whether the total reference } \\
\text { limb ulcerated area was less than or equal to } 10 \mathrm{~cm}^{2} \text {, or greater than } 10 \mathrm{~cm}^{2} \text {. } \\
\text { Trial authors informed us that sealed envelopes were used. Standard data } \\
\text { checks undertaken for the IPD meta-analysis suggested that allocation con- } \\
\text { cealment was satisfactory. }\end{array}$ \\
\hline
\end{tabular}

$\begin{array}{ll}\begin{array}{l}\text { Incomplete outcome data } \\ \text { (attrition bias) }\end{array} & \begin{array}{l}116 \text { people recruited and } 112 \text { people analysed. Of the } 4 \text { people excluded from } \\ \text { the analysis, } 3 \text { did not provide any follow-up data and one was recruited in er- } \\ \text { All outcomes }\end{array} \\ \text { ror. The } 4 \text { excluded patients were not reinstated for the IPD meta-analysis. }\end{array}$

Author correspondence.

Blinded outcome assess- High risk ment (healing)

Baseline comparability Low risk Median ulcer area and duration fairly well balanced. Analysis by Cox model.

\section{Polignano 2004a}

Methods

RCT (multicentred), computerised randomisation list generated remotely, block randomisation used. Intended sample of 100 patients not recruited because of changing practice in the study clinics, so study was underpowered to detect between-group differences in healing outcomes.

Participants

68 patients ( 1 wound each), inpatients and outpatients, all ambulant, recruited from 4 study centres in Italy.

Inclusion criteria: patient age $\geq 18$ years; venous ulceration confirmed by Doppler.

Exclusion criteria: pregnancy; $\mathrm{ABPI}<0.8$; rheumatoid vasculitis; diabetic foot ulceration; malignant ulceration; clinically infected ulcer; excessive exudate; ulcer area $>10 \mathrm{~cm}^{2}$.

Number of male:female patients: Group 1: 16:23; Group 2: 10:19.

Mean \pm SD, median (range) patient age in years: Group 1: $68.4 \pm 13.9,72.0$ (23.0-89.0); Group 2: $68.6 \pm$ 9.6, 69.0 (43.0-87.0).

Mean \pm SD, median (range) baseline ulcer area (length $x$ width) in $\mathrm{cm}^{2}$ : Group 1: $10.1 \pm 11.4,5.5$

(0.8-52.5); Group 2: $9.3 \pm 12.8$, 3.6 (0.3-47.5).

Number (\%) patients with baseline ulcer duration $<7$ days vs 7 days-1 month vs $1-6$ month vs $6-12$ months:

Group 1: 3 (8\%) vs $16(41 \%)$ vs 5 (13\%) vs $15(38 \%)$; Group 2: 2 (7\%) vs $16(55 \%)$ vs $5(17 \%)$ vs $6(21 \%)$.

Interventions

Bandages changed at least weekly in both groups. 
Polignano 2004a (Continued)

Group 1: 4LB (Profore) $(n=39)$;

Group 2: Unna's Boot, comprising zinc oxide paste bandage (Viscopaste) plus elastic cohesive bandage

(Tensoplast) $(n=29)$.

Outcomes

Patients followed-up until healing or 24 weeks. Ulcer area measured every 4 weeks.

Number (\%) patients with complete healing at 24 weeks: Group 1: 29/39 (74\%); Group 2: 19/29 (66\%), P

$=0.42$. Estimate of difference between proportions healed $0.09(95 \% \mathrm{Cl}-0.13-0.31)$.

Estimate from Cox proportional hazards model including terms for bandage type, baseline ulcer area and baseline ulcer duration: HR $1.62(95 \% \mathrm{Cl} 0.87-3.02), \mathrm{P}=0.13$. Baseline ulcer area had a significant effect on healing with larger ulcers taking longer to heal $(P=0.01)$, but ulcer duration did not have a significant effect $(P=0.12)$.

Kaplan-Meier estimate of median days to healing: Group 1: 53 (95\% Cl 35-84). Group 2: 56 (95\% Cl 49-84).

Mean \pm SD, median (range) percentage reduction in ulcer area (estimated by ([Initial ulcer area - final area]/initial area) x 100) at 24 weeks: Group 1: 79.1 \pm 65.7, 100.0 (-283.3-100.0); Group 2: $24.6 \pm 165.5$, 100.0 (-489.3-100.0), $\mathrm{P}=0.30$.

Mean \pm SD, median (range) percentage reduction in ulcer area/day (estimated by dividing percentage reduction by number of days in trial): Group 1: $2.3 \pm 3.7,1.9$ (-13.5-14.3); Group 2: $0.0 \pm 6.3,1.3$ (-22.2-7.7), P value not reported.

The between-group difference for change in pain score from baseline to final assessment (assessed with visual analogue scale) was not significant $(P=0.32)$.

Number (\%) of patients experiencing no change in pain vs decrease in pain vs increase in pain: Group 1 ( $n=34): 12(35 \%)$ vs $21(62 \%)$ vs $1(3 \%)$; Group $2(n=24): 3(13 \%)$ vs $19(79 \%)$ vs $2(8 \%)$.

\section{Notes}

\section{A nurse applied bandages in accordance with manufacturers' instructions.}

Withdrawals: 3 patients/group discontinued treatment due to an unassociated medical condition; 1 patient/group discontinued because of an adverse event (intolerance to treatment and pain).

Numbers allocated to each group did not appear to be well balanced (57\% in group 1). Trial author explained that this was because difficulties with recruitment (see methods, above).

Components of Unna's Boot and details of randomisation and allocation concealment confirmed by trial authors.

\section{Risk of bias}

\begin{tabular}{|c|c|c|}
\hline Bias & Authors' judgement & Support for judgement \\
\hline $\begin{array}{l}\text { Random sequence genera- } \\
\text { tion (selection bias) }\end{array}$ & Low risk & $\begin{array}{l}\text { Author provided clarification: "... the allocation was done by a remote com- } \\
\text { puter. The list of randomisation the computer provided was sealed in an enve- } \\
\text { lope and opened when a patient was recruited...". }\end{array}$ \\
\hline $\begin{array}{l}\text { Allocation concealment } \\
\text { (selection bias) }\end{array}$ & Low risk & $\begin{array}{l}\text { Author provided clarification: "... the allocation was done by a remote com- } \\
\text { puter. The list of randomisation the computer provided was sealed in an enve- } \\
\text { lope and opened when a patient was recruited ...". }\end{array}$ \\
\hline $\begin{array}{l}\text { Incomplete outcome data } \\
\text { (attrition bias) } \\
\text { All outcomes }\end{array}$ & Low risk & $\begin{array}{l}\text { Analysis for healing by intention to treat though others e.g. pain, only on a sub- } \\
\text { set of participants. Difficult to judge completeness of continuous outcome da- } \\
\text { ta. } \\
68 \text { people recruited and healing data reported on all } 68 \text {. }\end{array}$ \\
\hline
\end{tabular}


Polignano 2004a (Continued)

Blinded outcome assess- Unclear risk No details provided. ment (healing)

Baseline comparability High risk

Ulcers slightly larger in Group 1 at baseline; duration of ulcer data only presented categorically, however, appears that more people with ulcers of longer duration in Group 1.

\section{Polignano 2004b}

Methods RCT (multicentred) pilot study.

Participants

56 patients with venous leg ulcers recruited from 3 study centres in Italy

Inclusion criteria: venous leg ulcer with surface area $>2 \mathrm{~cm}^{2}$ but $<10 \mathrm{~cm}$ in any dimension; $A B P I>0.8$; ankle circumference $18-30.5 \mathrm{~cm}$.

Exclusion criteria: "champagne-bottle" shaped legs; severe arthritis; history of poor concordance with therapy; hypersensitivity to any study material; immobility; systemic antibiotic use; infected or mixed aetiology ulcers; recent history of participants in other clinical investigations.

Number of male:female patients: Group 1: 8:21 Group 2: 13:14.

Mean \pm SD (range) patient age in years: Group 1: $70.8 \pm 10.5$ (42-89); Group 2: $67.3 \pm 13.6$ (38-92).

Mean \pm SD (range) body weight in kg: Group 1: $75.2 \pm 13.8$ (55-120); Group 2: $78.3 \pm 15.9$ (53-110).

Mean \pm SD (range) height in cm: Group 1: $167 \pm 9$ (155-190); Group 2: $168 \pm 11$ (146-188).

Mean \pm SD (range) ABPI: Group 1: $1.0 \pm 0.1$ (0.80-1.10); Group 2: $1.0 \pm 0.1$ (0.9-1.20).

Number (\%) patients with major clinical condition present: Group 1: 16/29 (55\%): Group 2: 5/27 (19\%).

Number (\%) patients with history of allergy: Group 1: 1/29 (3\%): Group 2: 2/27 (7\%).

Number (\%) patients with abnormalities present at clinical examination: Group 1: 9/29 (31\%): Group 2: $3 / 27(11 \%)$.

Mean \pm SD (range) baseline ulcer surface area in $\mathrm{cm}^{2}$ : Group 1: $9.7 \pm 9.4(0.4-40.0)$; Group 2: $9.3 \pm 8.1$ (0.49-30.8).

Mean \pm SD (range) baseline maximum ulcer diameter in cm: Group 1: $4.6 \pm 2.9$ (1.0-11.8); Group 2: $4.4 \pm$ $2.5(1.2-12.5)$.

Number (\%) patients with baseline ulcer duration $\leq 6$ months vs > 6 months: Group 1: $10 / 29$ (34\%) vs 19/29 (66\%); Group 2: 11/27 (41\%) vs 16/27 (59\%).

Number (\%) patients with baseline exudate level assessed as none vs mild vs moderate heavy:

Group 1: $7 / 29$ (24\%) vs $12 / 29(41 \%)$ vs $9 / 29(31 \%)$ vs $1 / 29(3 \%)$;

Group 2: $8 / 27$ (30\%) vs $9 / 27(33 \%)$ vs $7 / 27(26 \%)$ vs $3 / 27(11 \%)$.

Interventions

All patients received wound cleansing as required and application of gauze.

Group 1: SSB (Comprilan) ( $\mathrm{n}=29)$.

Group 2: SurePress Comfort (consists of 2 latex-free knee-high nylon and spandex stockings; a medium compression overstocking and light compression understocking designed to provide a high compression system overall). Can be applied by patients $(n=27)$.

Outcomes

Study duration 12 weeks with assessments at baseline then 4-weekly thereafter. Wounds measured at each visit using direct transparency tracing and photography.

Efficacy analysis based on all 56 patients. Safety analysis based on 53 patients (Group 1: 28, Group 2: 25), 3 patients excluded because they failed to attend the first interview.

Number (\%) patients with complete healing during 12-week study period: Group 1: 5/29 (17\%, $95 \% \mathrm{Cl}$ 4-45\%); Group 2: $12 / 27$ (44\%, 95\% Cl 21-71\%), $\mathrm{P}=0.027$.

Mean \pm SD $(95 \% \mathrm{Cl})$ days to healing: Group 1: $101 \pm 7$ (87-114); Group 2: $72 \pm 5$ (62-82), $\mathrm{P}=0.027$ (log rank test).

Mean \pm SD (range) local ulcer pain intensity under compression assessed at the start of treatment using $100 \mathrm{~mm}$ visual analogue scale: Group 1: $29.5 \pm 34.0$ (0.0-100.0); Group 2: $33.4 \pm 31.8$ (0.0-100.0). 
Polignano 2004b (Continued)

Local ulcer pain decreased significantly more in Group 2 (70\% decrease) vs Group 1 (less than 20\% decrease) $(P=0.017$, unpaired t-test $)$.

Number of patients with onset of new venous ulcers during the study period: Group 1: 2; Group 2: 3.

Comfort while wearing compression (assessed with 4-point verbal rating scale at weeks 2-4): Group 2 had superior comfort during entire study period compared with Group $1(P=0.038$, full statistics not reported in paper).

Self-rated patient concordance with compression (assessed using questions rated on a 3-point scale at weeks 2-4): no significant difference between groups, most patients reported good concordance.

Percentage of patients reporting good concordance (range over assessment week period): Group 1: 80.8\%-92.9\%; Group 2: 92.3-100.0\%.

Notes

Number (\%) of patients withdrawing from study overall with following reasons - adverse event; inefficacy (development of new ulcer); consent withdrawn; lost to follow-up: Group 1: 11/29 (38\%) 5; 4; 1; $;$; Group 2: $4 / 27(15 \%)$ 0; $1 ; 1 ; 2$.

1 adverse event in Group 1 considered to be potentially related to compression therapy (bullous dermatitis).

Withdrawals from either group because of inefficacy treated with an alterative compression system.

Higher proportion of patients in Group 1 had a major clinical condition or abnormalities present at baseline clinical examination. Difficult to judge whether these variables could have influenced healing, as no further details provided.

\section{Risk of bias}

\begin{tabular}{lll} 
Bias & Authors' judgement & Support for judgement \\
\hline $\begin{array}{l}\text { Random sequence genera- } \\
\text { tion (selection bias) }\end{array}$ & Unclear risk & $\begin{array}{l}\text { "This study was a multicentre (3) open label comparative randomised parallel } \\
\text { group pilot trial". No further detail given. }\end{array}$ \\
\hline $\begin{array}{l}\text { Allocation concealment } \\
\text { (selection bias) }\end{array}$ & Unclear risk & No further details provided. \\
\hline $\begin{array}{l}\text { Incomplete outcome data } \\
\text { (attrition bias) } \\
\text { All outcomes }\end{array}$ & Low risk & $\begin{array}{l}\text { "Data were analysed according to the intention to treat principle and includ- } \\
\text { ed all patients recruited into the study. The last observation carried forward } \\
\text { method was also used ... Efficacy analysis was based on the ITT data set of } 56 \\
\text { patients... 3 patients (2 in the test group and } 1 \text { in the reference group) failed } \\
\text { to report for the first interview so were excluded from the safety data set. The } \\
\text { safety data set thus included } 53 \text { patients, 25 in the test group and } 28 \text { in the ref- } \\
\text { erence group". }\end{array}$ \\
\hline
\end{tabular}

Blinded outcome assess- Unclear risk No details provided. "Acetate tracings and photographs of the ulcer were tak-
ment (healing) en at each visit to evaluate the proportion of the wound that was healing".

Baseline comparability Unclear risk Mean ulcer area looked similar, but no median data provided. Impossible to
judge comparability of ulcer duration as only presented as categorical data.

\section{Rubin 1990}

\begin{tabular}{ll}
\hline Methods & RCT (multicentred), outpatient setting, USA. \\
\hline Participants & $\begin{array}{l}36 \text { consecutive ambulatory patients with lower-extremity chronic venous stasis ulceration recruited } \\
\text { from hospital clinics. }\end{array}$
\end{tabular}


Rubin 1990 (Continued)

Exclusion criteria: history of non-compliance; $\mathrm{ABPI}<0.8$; history of risk factors such as collagen vascular disease, uncontrolled diabetes, ongoing dermatological disorders; and chronic corticosteroid therapy.

Mean (range) baseline ulcer area in $\mathrm{cm}^{2}$ : Group 1: 76.0 (0.02-600.0); Group 2: 32.2 (6.0-270.0).

\begin{abstract}
Interventions
All patients: instructed regarding the need for leg elevation, signs and symptoms of wound complications and the need for concordance with follow-up. Dressings changed weekly or twice weekly by hospital-based nursing staff, in accordance with prescription. Wounds cleansed with $20 \%$ poloxamer 188 solution (Shur-Cleans). Reapplication of elastic bandage performed, as necessary, between dressing changes, at home or at the clinic.

Group 1: Unna's boot (gauze bandage impregnated with glycerin, zinc oxide and calamine lotion) plus elastic bandage applied from toes to knee ( $n=19$ patients).

Group 2: polyurethane foam dressing (Synthaderm) plus elastic bandage applied from toes to knee ( $n=$ 17 patients).
\end{abstract}

Outcomes

Number (\%) of patients with complete healing at 12 months: Group 1: 18/19 (95\%); Group 2: 7/17 (41\%) $(\mathrm{P}<0.005$, chi-squared test).

Mean healing rate in $\mathrm{cm}^{2} /$ day: Group 1: 0.5; Group 2: 0.07 ( $P=0.004$, Student's t-test).

Number (\%) patient withdrawals from treatment during 12-month trial: Group 1: 0/19 (0\%); Group 2: 9/17 (53\%) (all Group 2 withdrawals were because of malodorous drainage resulting from autolytic debridement).

6 of the 9 patients who withdrew in Group 2 experienced enlargement of the ulcer during the trial.

Notes
Wounds measured by same investigator at each dressing change using tracing and planimetry (exact methods not specified).

Elastic bandages (used by all patients) appear to have been used as a retaining wrap; comments in the discussion section suggest that these bandages did not provide compression.

\title{
Risk of bias
}

\begin{tabular}{lll}
\hline Bias & Authors' judgement & Support for judgement \\
\hline $\begin{array}{l}\text { Random sequence genera- } \\
\text { tion (selection bias) }\end{array}$ & Unclear risk & No details provided. Merely described the trial as "randomised". \\
\hline $\begin{array}{l}\text { Allocation concealment } \\
\text { (selection bias) }\end{array}$ & Low risk & $\begin{array}{l}\text { "Each patient was randomised by the study co-ordinator to either a } \\
\text { polyurethane foam dressing or Unna's boot dressing treatment protocol. The } \\
\text { study co-ordinator did not see the randomisation card and was therefore } \\
\text { blinded as to the treatment cohort". }\end{array}$ \\
\hline $\begin{array}{l}\text { Incomplete outcome data } \\
\text { (attrition bias) }\end{array}$ & Unclear risk & $\begin{array}{l}\text { All randomised patients contributed healing data, however, less clear for con- } \\
\text { tinuous outcomes whether all participants were included. } 9 \text { people classed } \\
\text { as withdrawals in Group 2, none in Group 1. It is somewhat unclear whether } \\
\text { withdrawal meant withdrawal from trial treatment but trial outcomes were } \\
\text { observed, or merely that patients were withdrawn from follow-up but included } \\
\text { in the denominator as unhealed. }\end{array}$
\end{tabular}

\begin{tabular}{lll}
\hline $\begin{array}{l}\text { Blinded outcome assess- } \\
\text { ment (healing) }\end{array}$ & Unclear risk & No details provided. \\
\hline Baseline comparability & High risk & Mean area only presented, however, mean area much larger in Group 1. \\
\hline
\end{tabular}


Scriven 1998

\section{Methods}

RCT (block method with stratification by ulcer area $\leq 10 \mathrm{~cm}^{2} \mathrm{vs}>10 \mathrm{~cm}^{2}$ ). Patients with bilateral ulceration had each limb randomised separately. Setting: leg ulcer clinic, UK.

\section{Participants}

53 ambulant patients with 64 ulcerated limbs recruited from a venous ulcer assessment clinic. Inclusion criteria: active lower limb ulceration; venous aetiology defined as venous reflux $>0.5 \mathrm{~s}$ duration and $\mathrm{ABPI}>0.8$.

Exclusion criteria: not stated.

Number of male:female patients (breakdown/group not reported): 20:33.

Median (range) patient age in years: Group 1: 70 (45-91); Group 2: 73 (36-93).

Median [mean] (range) baseline ulcer area in $\mathrm{cm}^{2}$ : Group 1: 13.3 [49.6] (2-378); Group 2: 8.3 [19.1] (2-104).

Number (\%) limbs with baseline ulcer area > $10 \mathrm{~cm}^{2}$ : Group 1: 21/32 (66\%); Group 2: 14/32 (44\%). Median (range) baseline ulcer duration in months: Group 1: 13 (1-480); Group 2: 21 (3-360). elastic cohesive bandage (Coban). Bandages replaced at each dressing change ( $n=32$ limbs).

Group 2: SSB comprising: orthopaedic wool (Velband); SSB applied with 50\% stretch and 50\% overlap between turns (Rosidal K); and elastic cohesive bandage applied without stretch (Coban). Bandages washed, reused, and replaced after 20 washes ( $n=32$ limbs).

All patients: compression therapy applied for 12 weeks. Standardised bandage application carried out by nursing staff trained and experienced in compression bandaging. Primary dressing a simple non-adherent dressing covered with gauze. Bandages changed once a week unless strike through of exudate. After withdrawal (either due to ulcer deterioration during the trial or failure to heal at 12 weeks), patients could opt to receive the alternative bandage. Post-healing, class II compression stockings were provided.

\section{Outcomes}

Kaplan-Meier estimate of limbs with complete healing at 1 year: Group 1: 55\%; Group 2: 57\% (P=1.0, log rank test).

Number of adverse events (description): Group 1: 1 (minor haemorrhagic blistering of toes distal to bandage); Group 2: 4 (2 pressure-induced iatrogenic ulceration, 2 maceration).

Number (\%) limbs withdrawn (reasons): Group 1: 1/32 (3\%) (did not attend follow-up clinics 1); Group 2: 2/32 (6\%) (died 1, did not attend follow-up clinics 1 ).

Unit cost and estimated cost of treatment over 6 months, based on costs of bandage systems only (GBP, price year not stated): Group 1: GBP 15.10 and GBP 392.60; Group 2: GBP 7.10 and GBP 184.56.

\section{Notes}

Ulcer area measured every 2 weeks using transparency tracing and computerised planimetry. Ulcer healing defined as full re-epithelialisation. Limb volume assessed during the trial. Ankle sub-bandage pressure assessed using the Oxford Pressure Monitor. Addition of the unstretched cohesive bandage to the SSB system (Group 2) resulted in a pressure increase of $11.5 \mathrm{mmHg}$.

Trial authors' analysis conducted on an ITT basis (the 3 withdrawals were included). Data from both study arms were merged and subject to chi-squared analysis to examine association between healing and the following: baseline ulcer area $>10 \mathrm{~cm}^{2}$; ulcer duration > 6 months; previous DVT; and presence of deep venous reflux. No statistically significant associations were detected.

Ulcer area larger in Group 1 at baseline.

Since limbs are not independent with respect to healing, this may have influenced the results.

\section{Risk of bias}

\begin{tabular}{lll}
\hline Bias & Authors' judgement & Support for judgement \\
\hline $\begin{array}{l}\text { Random sequence genera- } \\
\text { tion (selection bias) }\end{array}$ & Low risk & $\begin{array}{l}\text { Limb randomisation achieved using sealed envelopes that specified the type } \\
\text { of bandage to be applied, determined by a block randomisation method. Stan- }\end{array}$
\end{tabular}


Scriven 1998 (Continued)

dard data checks undertaken for the IPD meta-analysis suggested that generation of the random sequence was satisfactory.

\begin{tabular}{ll}
\hline $\begin{array}{l}\text { Allocation concealment } \\
\text { (selection bias) }\end{array}$ & Low risk \\
\end{tabular}

Incomplete outcome data Low risk

(attrition bias)

All outcomes

\begin{abstract}
Information from trial report:
"During the study period one patient died after two attendances and two patients repeatedly failed to attend ... these two patients represented two ulcerated limbs randomised to 4 layer bandage one limb and SSB one limb. They were subsequently considered as treatment failures and are thus included in the analysis of results on an intention to treat basis".

Healing data available for all patients included in the patient level meta-analysis. Limbs, rather than patients, were the unit of allocation. For 12 patients with both limbs recruited to the trial, the limb with the smaller area of ulceration was deleted from the IPD meta-analysis data set.
\end{abstract}

\begin{tabular}{lll}
\hline $\begin{array}{l}\text { Blinded outcome assess- } \\
\text { ment (healing) }\end{array}$ & High risk & $\begin{array}{l}\text { No details in study report, however, trial authors confirmed that outcome as- } \\
\text { sessment was not blinded. }\end{array}$ \\
\hline Baseline comparability & Low risk & $\begin{array}{l}\text { Examination of IPD indicated satisfactory balance of baseline variables across } \\
\text { groups. }\end{array}$ \\
\hline
\end{tabular}

Szewczyk 2010

Methods RCT performed at hospital outpatient clinic in Bydgoszcz, Poland.

\title{
Participants
}

46 patients recruited from a hospital-based venous leg ulcer outpatient clinic.

Inclusion criteria: presence of venous leg ulceration confirmed by duplex scan and ABPI 0.9-1.3; maximum ulcer surface area $15 \mathrm{~cm}^{2}$.

Exclusion criteria: non-venous or mixed ulcer aetiology; pregnancy; presence of diabetes, lower limb atherosclerosis, arterial hypertension, cardiovascular insufficiency, rheumatoid arthritis or other autoimmune diseases.

Number of male:female patients: Group 1: 2:13; Group 2: 4:12; Group 3: 4:11.

Mean \pm SD patient age in years: Group 1: $66.4 \pm 9.2$; Group 2: $67.5 \pm 9.0$; Group 3: $65.9 \pm 9.2$.

Mean \pm SD ulcer surface area in $\mathrm{cm}^{2}$ : Group 1: $4.7 \pm 4.2$; Group 2: $5.3 \pm 3.9$; Group 3: $6.0 \pm 4.0$.

Interventions Group 1: knee-length, class II compression stockings fitted according to patient's limb dimensions (Maxis, PPH Real, Poland) ( $\mathrm{n}=15$ patients).

Group 2: 2-component compression bandage system (ProGuide, Smith \& Nephew, UK), comprising wool layer and elastic bandage (trial report states SSB, but this does not agree with manufacturer's description of ProGuide). Bandages applied using a spiral technique and changed at least twice weekly. Bandages applied to achieve ankle sub-bandage pressure of $40 \mathrm{~mm} \mathrm{Hg}$, and $20 \mathrm{~mm} \mathrm{Hg}$ at widest part of the calf ( $n=16$ patients).

Group 3: 4LB (Profore, Smith \& Nephew, UK), bandages applied using a spiral technique and changed at least twice weekly, to achieve ankle sub-bandage pressure of $40 \mathrm{~mm} \mathrm{Hg}$, and $20 \mathrm{~mm} \mathrm{Hg}$ at the widest part of the calf ( $n=15$ patients). 
All patients: received hydrotherapy and mechanical wound care (unclear whether this information referred to ulcer debridement); at each clinic visit the affected limb and peri-ulcer skin were washed, moisturised and foam or hydrocolloid dressing applied according to requirements.

Outcomes $\quad$ Number (\%) of patients with complete healing at 12 weeks: Group 1: 8/15 (53\%); Group 2: 10/16 (63\%); Group 3: $9 / 15(60 \%)$.

Average (presume mean) \% change in ulcer surface area at 12 weeks: Group 1: -83.1\%; Group 2: -98.1\%; Group 3: -93.9\% (trial authors reported $\mathrm{P}>0.05$ for between-group differences).

Average (presumed mean) healing rate in $\mathrm{cm}^{2} /$ week assessed at 12 weeks: Group 1: 0.44; Group 2: 0.55; Group 3: 0.63 (trial authors reported $P>0.05$ for between-group differences).

\section{Notes}

Trial authors stated that compression bandages were applied by a qualified and trained nurse, but no further details about skill, experience or training provided.

In Groups 2 and 3, ankle sub-bandage pressure measured using a Kikuhime manometer. Unclear whether this was assessed at every bandage change.

Ulcer surface area assessed using digital planimetry (Visitrak, Smith \& Nephew, UK) at baseline and every 2 weeks thereafter.

\section{Risk of bias}

\begin{tabular}{lll}
\hline Bias & Authors' judgement & Support for judgement \\
\hline $\begin{array}{l}\text { Random sequence genera- } \\
\text { tion (selection bias) }\end{array}$ & Unclear risk & "... patients ... were randomised into three groups". \\
\hline $\begin{array}{l}\text { Allocation concealment } \\
\text { (selection bias) }\end{array}$ & Unclear risk & No details provided. \\
\hline $\begin{array}{l}\text { Incomplete outcome data } \\
\text { (attrition bias) } \\
\text { All outcomes }\end{array}$ & Low risk & No mention of study withdrawals, but the report of complete healing ap- \\
\hline $\begin{array}{l}\text { Blinded outcome assess- } \\
\text { ment (healing) }\end{array}$ & Unclear risk & No details provided. \\
\hline $\begin{array}{l}\text { Baseline comparability } \\
\text { Unclear risk }\end{array}$ & Median and ranges not reported for ulcer surface area, so comparability diffi- \\
\hline
\end{tabular}

Taradaj 2007

Methods RCT in an out-patient clinic in Poland.

Participants 73 patients with venous leg ulcers recruited after surgery for ligation and stripping (Babcock procedure) on saphenous or sagittal veins.

Inclusion criteria: venous leg ulcer confirmed with Doppler ultrasound.

Exclusion criteria: presence of diabetes, atherosclerosis or rheumatoid arthritis; steroid treatment; metal implants present at ultrasound application site; ulcer aetiology other than venous.

Number of male:female patients: Group 1: 9:15; Group 2: 9:16; Group 3: 13:11.

Mean \pm SD (range) patient age in years: Group 1: $62.0 \pm 9.8$ (47-85); Group 2: $61.6 \pm 8.3$ (43-78); Group 3: $62.3 \pm 9.5(40-79)$. 
Taradaj 2007 (Continued)

Number of patients with superficial vs superficial and deep venous insufficiency: Group 1: 9 vs 15; Group 2: 9 vs 16; Group 3: 9 vs 15.

Mean \pm SD ulcer area in $\mathrm{cm}^{2}$ : Group 1: $26.5 \pm 17.0$; Group 2: $24.4 \pm 12.9$; Group 3: $22.0 \pm 15.5$.

Mean \pm SD (range) ulcer duration in weeks: Group 1: $33 \pm 27$ (4-124); Group 2: $36 \pm 39$ (6-176); Group 3: 32 $\pm 35(2-120)$.

Interventions

Group 1: moist normal saline dressing, ultrasound therapy and pharmacotherapy (diosmin $450 \mathrm{mg}$ and hesperidin $50 \mathrm{mg}$ combined as proprietary preparation (Detralex) ( $n=24$ patients).

Group 2: moist normal saline dressing, 2-component compression system comprising an elastic bandage (Sigvaris) applied at $30 \mathrm{~mm} \mathrm{Hg}$ ankle pressure for superficial venous insufficiency, and $40 \mathrm{~mm} \mathrm{Hg}$ for superficial and deep venous insufficiency (unclear whether pressure was verified) plus stocking (no further details of this) and pharmacotherapy as above ( $n=25$ patients).

Group 3: moist normal saline dressing plus pharmacotherapy as above ( $n=24$ patients).

All patients: treatment duration 7 weeks.

Outcomes $\quad$ Mean \% change in ulcer area (relative to baseline) at 7 weeks: Group 1: -53.6\%; Group 2: -69.4\%; Group 3: $-62.6 \%$ ( $P>0.05$ for all 3 comparisons between groups).

Mean percentage change in ulcer area/week (NB: values read from figure): Group 1: -7.6\%; Group 2: -9.9\%; Group 3: -8.9\% ( $P>0.05$ for all 3 comparisons between groups).

Mean \pm SD ulcer area in $\mathrm{cm}^{2}$ at 7 weeks (NB: comparisons are within group vs baseline): Group 1: $14.1 \pm$ $11.7(\mathrm{P}=0.00002)$; Group 2: $8.8 \pm 10.0(\mathrm{P}=0.00001)$; Group 3: $11.4 \pm 14.1(\mathrm{P}=0.00002)$.

No secondary outcomes reported.

No report of withdrawals from the trial.

Notes

Ulcers assessed at baseline and weekly during treatment using a digitiser combined with computerised planimetry. In addition, ulcers were photographed (frequency and other details of this unclear).

No information on experience or skill of care providers.

Patients were the unit of randomisation.

Trial report was in Polish; data were extracted with the assistance of a translator.

\section{Risk of bias}

\begin{tabular}{lll}
\hline Bias & Authors' judgement & Support for judgement \\
\hline $\begin{array}{l}\text { Random sequence genera- } \\
\text { tion (selection bias) }\end{array}$ & Unclear risk & From translator: " . . random assignment... ". \\
& $\begin{array}{l}\text { Comment: no randomisation method specified. Authors did not state whether } \\
\text { patients were randomised before or after surgery. }\end{array}$ \\
\hline $\begin{array}{l}\text { Allocation concealment } \\
\text { (selection bias) }\end{array}$ & Unclear risk & No details. \\
\hline $\begin{array}{l}\text { Incomplete outcome data } \\
\text { (attrition bias) } \\
\text { All outcomes }\end{array}$ & Unclear risk & $\begin{array}{l}\text { No report of withdrawals, and not clear from report whether all patients in- } \\
\text { cluded in the analyses. }\end{array}$ \\
\hline $\begin{array}{l}\text { Blinded outcome assess- } \\
\text { ment (healing) }\end{array}$ & Unclear risk & No details. \\
\hline
\end{tabular}


Taradaj 2007 (Continued)

Baseline comparability Unclear risk Groups appear comparable, however, more meaningful median values were not reported for ulcer area and duration (mean values presented).

Taradaj 2009

Methods RCT conducted in 3 study centres in Poland in an outpatient setting.

Participants

80 patients randomised.

Inclusion criteria: venous leg ulceration confirmed by duplex scan and ABPI.

Exclusion criteria: $\mathrm{ABPI}<0.9$; presence of diabetes, arthritis or arrhythmia; pregnancy; previous ulcer surgery; treatment with steroids; skin infection.

Number of male:female patients: Group 1: 15:25; Group 2: 18:22 (NB: discrepancy in numbers in Group 2 between main text and tabulated information; numbers here taken from main text).

Mean \pm SD (range) patient age in years: Group 1: $63.4 \pm 8.9$ (40-80); Group 2: $62.0 \pm 8.3$ (43-80).

Number of patients with superficial only vs superficial plus deep venous reflux: Group 1: 22 vs 18; Group 2: 22 vs 18.

Mean \pm SD (range) ulcer surface area in $\mathrm{cm}^{2}$ : Group 1: $20.6 \pm 14.0$ (6.8-39.0); Group 2: $20.3 \pm 9.7$ (10.2-34.4).

Mean \pm SD (range) ulcer volume in $\mathrm{cm}^{3}$ : Group 1: $3.7 \pm 4.0$ (0.2-6.9); Group 2: $3.3 \pm 4.2$ (0.3-7.9).

Mean \pm SD (range) ulcer duration in months: Group 1: $30.5 \pm 23.3$ (2-100); Group 2: $30.1 \pm 25.1$ (4-98).

Interventions

Group 1: compression stockings (Sigvaris 702, Gianzoni \& Cie AG, Switzerland) providing ankle pressure 25-32 $\mathrm{mm} \mathrm{Hg}$. Stockings applied every morning at outpatient clinic, worn all day (10-12 h) and removed at night. Patients also received Detralex (diosmin $450 \mathrm{mg}$ plus hesperidin $50 \mathrm{mg}$ ), 2 tablets daily). Patients treated in hospital dermatology department. 2-month duration of treatment ( $n=40$ patients).

Group 2: 2 SSBs applied in a spiral configuration on the foot and a figure-of-8 configuration on the calf. Bandages worn during the day and removed at night. Ankle pressures standardised using a Kikuhime manometer (30-35 mm Hg for superficial vein reflux and 35-40 mm Hg for superficial plus deep venous reflux). Patients received drug therapy as above. Patients treated in a medical university dermatology department. 2-month duration of treatment ( $n=40$ patients).

All patients: ulcers bathed daily with potassium permanganate solution, then covered with moist normal saline dressings as well as compresses of fibrolan, chloramphenicol and colistin; dressings changed daily at the clinic.

Outcomes Number (\%) of patients with complete healing at 2 months: Group 1: 15/40 (37.5\%); Group 2: 5/40 (12.5\%) ( $\mathrm{P} \leq 0.001$, from trial authors' report).

Number (\%) of patients with isolated superficial venous reflux with complete healing at 2 months: Group 1: 10/22 (45.5\%); Group 2: 4/22 (18.2\%) ( $\mathrm{P} \leq 0.01$, from trial authors' report).

Number (\%) of patients with superficial plus deep venous reflux with complete healing at 2 months: Group 1: 5/18 (27.8\%); Group 2: 1/18 (5.6\%) ( $\mathrm{P} \leq 0.01$, from trial authors' report).

Mean \% change in ulcer surface area at 2 months: Group 1: - $61.5 \%$ : Group 2: - $23.7 \%$ ( $\mathrm{P} \leq 0.01$, from trial authors' report).

Mean \% change in ulcer volume at 2 months: Group 1: $-82.0 \%$ : Group 2: $-40.0 \%$ ( $\mathrm{P} \leq 0.01$, from trial authors' report). 
Taradaj 2009 (Continued)

Notes

Ulcer surface area measured using transparency tracings; wound volume assessed with a micrometer. Both methods combined with a digitising tablet (Kurta XGT, Altek Inc, USA) and modified computer software (C-GEO). Measurements taken at baseline, then weekly.

Data were provided on absolute change in ulcer surface area and volume during the trial, but comparisons were within group relative to baseline and so are not reported here.

\section{Risk of bias}

\begin{tabular}{lll}
\hline Bias & Authors' judgement & Support for judgement \\
\hline $\begin{array}{l}\text { Random sequence genera- } \\
\text { tion (selection bias) }\end{array}$ & Low risk & $\begin{array}{l}\text { "Computer generated random numbers were sealed in sequentially numbered } \\
\text { envelopes and group allocation was independent of place and person deliver- } \\
\text { ing the treatment". }\end{array}$ \\
\hline $\begin{array}{l}\text { Allocation concealment } \\
\text { (selection bias) }\end{array}$ & Low risk & $\begin{array}{l}\text { "Computer generated random numbers were sealed in sequentially numbered } \\
\text { envelopes and group allocation was independent of place and person deliver- } \\
\text { ing the treatment". }\end{array}$ \\
\hline $\begin{array}{l}\text { Incomplete outcome data } \\
\text { (attrition bias) } \\
\text { All outcomes }\end{array}$ & Low risk & $\begin{array}{l}\text { No report of study withdrawals. Analyses of complete healing were based on } \\
\text { all randomised patients. }\end{array}$ \\
\hline $\begin{array}{l}\text { Blinded outcome assess- } \\
\text { ment (healing) }\end{array}$ & High risk & $\begin{array}{l}\text { "...person who assessed parameters of ulcers was not blinded to the thera- } \\
\text { py ... ". }\end{array}$ \\
\hline $\begin{array}{l}\text { Baseline comparability } \\
\text { Unclear risk }\end{array}$ & $\begin{array}{l}\text { Groups appeared comparable, however, more meaningful median values were } \\
\text { not reported for ulcer area and duration (mean values presented). }\end{array}$ \\
\hline
\end{tabular}

\section{Taylor 1998}

$\begin{array}{ll}\text { Methods } & \text { RCT with randomisation performed by minimisation of prognostic factors (age, sex, body mass index, } \\ \text { mobility, range of ankle movement, ulcer area, ulcer duration and living alone). Community setting, } \\ \text { Salford, UK. }\end{array}$

Participants 36 consecutive patients referred to UK leg ulcer clinic from GP.

Inclusion criteria: venous ulceration; ABPI > 0.8 .

Exclusion criteria: none stated.

Number of male:female patients: Group 1: 7:9; Group 2: 4:10.

Median (range) patient age in years: Group 1: 73 (28-85); Group 2: 77 (60-84).

Number of patients with full vs limited mobility: Group 1: 10 vs 6; Group 2: 7 vs 7.

Median (range) degrees of ankle movement: Group 1: 40 (20-65); Group 2: 40 (26-60).

Median (range) baseline ulcer area in $\mathrm{cm}^{2}$ : Group 1: 5.4 (0.4-74.8); Group 2: 4.2 (0.6-76.0).

Number of patients with ulcer duration < 6months vs > 6months: Group 1: 7 vs 9; Group 2: 9 vs 5.

Interventions

Group 1: 4LB based on Charing Cross system. Patients treated by a specialist nurse or a district nurse, both of whom were experienced in leg ulcer management and application of compression bandages. Patients with painful or sloughy ulcers initially received hydrocolloid as the primary dressing (Granuflex or Comfeel) and had twice weekly dressing changes. Otherwise a non-adherent dressing was used and bandages were changed weekly at the patient's home or at the community leg ulcer clinic $(n=18$ patients).

Group 2: continued with usual treatment by GP and district nurse. Patients treated 2-3 times weekly at their homes by their usual district nurse. A wide variety of preparations were used including different cleansing agents, dressings, topical applications, skin treatments and bandages (some of which could 
Taylor 1998 (Continued)

have provided compression). Application of high-compression bandaging was not permitted ( $\mathrm{n}=18 \mathrm{pa}$ tients).

All patients: those who healed within the trial period received class II compression stocking and were followed-up in the leg ulcer review clinics.

\begin{tabular}{|c|c|}
\hline \multirow[t]{12}{*}{ Outcomes } & $\begin{array}{l}\text { Number (\%) of patients with complete healing at } 12 \text { weeks (assuming losses did not heal, calculated by } \\
\text { review author): }\end{array}$ \\
\hline & Group 1:12/18 (66.7\%); Group 2: 3/18 (16.6\%). \\
\hline & Number (\%) of patients with complete healing at 12 weeks (study completers, as reported in paper): \\
\hline & Group 1: 12/16 (75\%); Group 2: 3/14 (21\%), P = 0.003 for difference between groups. \\
\hline & Median time to healing: Group 1: 55 days; Group 2: 84 days. \\
\hline & $\begin{array}{l}\text { Comparison of healing distributions using the Lee-Desu statistic suggested that patients in Group } 1 \\
\text { healed faster than those in Group } 2 \text { (overall comparison statistic } 8.603, P=0.0034 \text { ). }\end{array}$ \\
\hline & $\begin{array}{l}\text { Number (\%) patients who withdrew from trial (reasons): Group 1:2/18 (11\%) (died } 1 \text {, scabies } 1) \text {; Group } \\
\text { 2: } 4 / 18(22 \%) \text { (died 1, healed before treatment } 1 \text {, treated with 4LB 1, developed cellulitis } 1) \text {. }\end{array}$ \\
\hline & $\begin{array}{l}\text { Cost analyses took account of consumables, district nurse time (including travel) and mileage costs. Es- } \\
\text { timates are presented in GBP (price year not stated): }\end{array}$ \\
\hline & $\begin{array}{l}\text { Median (range) weekly treatment costs: Group 1: GBP } 17.26 \text { (13.45-20.16); Group 2: GBP } 21.07 \text { (8.71 - } \\
\text { 42.47) }(P=0.042) \text {. }\end{array}$ \\
\hline & Mean $(95 \% \mathrm{Cl})$ between-group difference in weekly treatment costs: GBP $6.45(1.22-11.68), P=0.042$ \\
\hline & $\begin{array}{l}\text { Median (range) whole trial costs: Group 1: GBP } 116.87 \text { (52.63-261.74); Group 2: GBP } 240.28 \\
\text { (74.65-588.05), } P=0.016 .\end{array}$ \\
\hline & Mean $(95 \% \mathrm{Cl})$ between-group difference in whole trial costs: GBP $113.51(29.71-197.31), \mathrm{P}=0.016$ \\
\hline Notes & $\begin{array}{l}\text { In patients with multiple ulcers, the total ulcerated area was studied. Ulcer area was measured weekly } \\
\text { using transparency tracing and computerised planimetry. }\end{array}$ \\
\hline
\end{tabular}

\section{Risk of bias}

\begin{tabular}{|c|c|c|}
\hline Bias & Authors' judgement & Support for judgement \\
\hline $\begin{array}{l}\text { Random sequence genera- } \\
\text { tion (selection bias) }\end{array}$ & Low risk & $\begin{array}{l}\text { "Eighteen patients were randomly allocated to each treatment group using } \\
\text { the method of minimisation of prognostic factors... ". }\end{array}$ \\
\hline $\begin{array}{l}\text { Allocation concealment } \\
\text { (selection bias) }\end{array}$ & Low risk & $\begin{array}{l}\text { We have assumed that the minimisation programme resulted in allocation } \\
\text { concealment. }\end{array}$ \\
\hline $\begin{array}{l}\text { Incomplete outcome data } \\
\text { (attrition bias) } \\
\text { All outcomes }\end{array}$ & High risk & $\begin{array}{l}\text { Authors did not undertake an ITT analysis; } 2 \text { people withdrew from Group } 1 \\
\text { and } 4 \text { from Group } 2 \text {, including } 1 \text { person who was not included in the analysis } \\
\text { because he/she received the Group } 1 \text { treatment. }\end{array}$ \\
\hline $\begin{array}{l}\text { Blinded outcome assess- } \\
\text { ment (healing) }\end{array}$ & Unclear risk & $\begin{array}{l}\text { "Weekly each patient had the perimeter of their ulcer traced onto an acetate } \\
\text { and the area measured using a computerised planimeter...". }\end{array}$ \\
\hline Baseline comparability & High risk & Ulcers in Group 1 had larger baseline area and were also of longer duration. \\
\hline
\end{tabular}


Travers 1992

\begin{tabular}{|c|c|}
\hline Methods & RCT (details of methods not provided). Setting: leg ulcer clinic, Nottingham, UK. \\
\hline Participants & $\begin{array}{l}\text { Recruited } 27 \text { patients attending leg ulcer clinic. } \\
\text { Inclusion criterion: venous ulcers (ABPI > 0.9). } \\
\text { Exclusion criteria: not stated. } \\
\text { Mean } \pm \text { SD patient age in years: Group 1: } 54 \pm 3 \text {; Group 2: } 59 \pm 4 . \\
\text { Mean } \pm \text { SD baseline ulcer area in mm2: Group 1: } 3097 \pm 1818 ; \text { Group 2: } 2304 \pm 1221 \\
\text { Mean } \pm \text { SD baseline ulcer duration in months: Group 1: } 23 \pm 7 \text {; Group 2: } 35 \pm 13 \text {. }\end{array}$ \\
\hline Interventions & $\begin{array}{l}\text { All patients: ulcers cleansed with sterile normal saline, then hydrocolloid primary dressing applied. } \\
\text { Bandages changed } 1-2 \text { times/week. } \\
\text { Group 1: single-component system consisting of elastic cohesive bandage (Panelast Acryl) applied } \\
\text { from foot to below-knee with } 50 \% \text { overlap ( } n=15 \text { patients). } \\
\text { Group 2: } 3 \text {-component system applied from foot to below-knee consisting of: zinc oxide and calamine } \\
\text { paste bandage (Calaband); non-adhesive elastic bandage (Tensopress) applied with } 50 \% \text { overlap and } \\
50 \% \text { stretch; and elasticated tubular bandage (Tensogrip) ( } n=12 \text { patients). }\end{array}$ \\
\hline
\end{tabular}

Outcomes

Mean \pm SE \% change relative to baseline ulcer area at 7 weeks (values taken from figure): Group 1: $-90 \pm$ 3; Group 2: $-83 \pm 5$ (authors report no statistically significant difference between groups using Student's t-test, but $P$ value not shown).

All patients completed the trial.

Notes

Ulcer area measured weekly using transparency tracing and computerised planimetry. The variability statistics used in the trial report were not specified, but presumed by the review authors to be standard deviation for baseline variables and standard error (shown on figure) for the outcome.

Sub-bandage ankle pressure measured with patients in a supine position using the Oxford Pressure Monitor. Average pressure at the start of treatment: Group 1: $50 \mathrm{mmHg}$; Group 2: $44 \mathrm{mmHg}$ (between-group difference reported as not significant by authors, but $\mathrm{P}$ value not shown). Average pressure after 1 week of treatment: Group 1: $23 \mathrm{mmHg}$; Group 2: $35 \mathrm{mmHg}(P<0.01)$. This suggested better maintenance of compression by the 3-component system.

The authors stated that costs of the bandages were equivalent, but no data were shown.

\section{Risk of bias}

\begin{tabular}{lll}
\hline Bias & Authors' judgement & Support for judgement \\
\hline $\begin{array}{l}\text { Random sequence genera- } \\
\text { tion (selection bias) }\end{array}$ & Unclear risk & ".. randomly allocated" - no further detail provided. \\
\hline $\begin{array}{l}\text { Allocation concealment } \\
\text { (selection bias) }\end{array}$ & Unclear risk & No further details provided. \\
\hline $\begin{array}{l}\text { Incomplete outcome data } \\
\text { (attrition bias) } \\
\text { All outcomes }\end{array}$ & Low risk & All 27 patients recruited "completed the trial". \\
\hline $\begin{array}{l}\text { Blinded outcome assess- } \\
\text { ment (healing) }\end{array}$ & Unclear risk & No detail provided. \\
\hline $\begin{array}{l}\text { Baseline comparability } \\
\text { Unclear risk }\end{array}$ & $\begin{array}{l}\text { Greater mean area at baseline in Group 1 and longer mean duration in Group 2, } \\
\text { however, mean data not useful as highly skewed. }\end{array}$ \\
\hline
\end{tabular}



power to detect $25 \%$ difference in healing rates at 12 weeks, at $5 \%$ significance level.

\section{Participants}

89 patients recruited from 2 study centres in Germany, 1 inpatient and 1 outpatient. Inclusion criterion: venous leg ulceration.

Exclusion criteria: $\mathrm{ABPI}<0.8$; rheumatoid vasculitis; ulceration of diabetic or malignant aetiology; use of corticosteroids; clinically-infected ulcer; circumferential ulcer.

Around $60 \%$ patients were female.

Mean patient age in years: Group 1: 67; Group 2: 70.

Mean BMI (kg/m²): Group 1: 27; Group 2: 28.

Number (\%) of ulcers with baseline duration > 6 months: Group 1: 23/44 (52\%); Group 2: 25/45 (56\%). Mean \pm SD, median (range) baseline ulcer area in $\mathrm{cm}^{2}$ : Group 1: $17.7 \pm 34.1,6.5$ (1.0-220.5); Group 2: 12.2 $\pm 14.8,6.6(1.8-70.7)$.

Interventions

All patients: ulcers cleaned with Ringer-Lactate Solution and covered with a polyurethane foam film dressing (Allevyn Hydrocellular).

Group 1: 4LB (Profore), reapplied weekly, or more often if required $(n=44)$.

Group 2: SSB comprising 2 bandages $10 \mathrm{~cm}$ wide. Bandages reapplied daily by patient, family member or nurse $(n=45)$.

When healed, patients were prescribed class II compression stockings and returned to the regular follow-up clinics.

Outcomes

Number (\%) patients healed at 12 weeks: Group 1: 13/44 (30\%); Group 2: 10/45 (22\%).

Kaplan-Meier estimate indicated that patients in Group 1 healed significantly faster than those in Group $2(P=0.03)$.

Cox regression: hazard ratio $2.9(95 \% \mathrm{Cl} 1.1-7.5)$ in favour of 4LB during the 12-week study period (with adjustment for bandage type, study centre, peri-wound skin condition, baseline ulcer area, baseline ulcer duration, and including an interaction term for study centre and bandage type); no statistically significant interaction between treatment and study centre $(P=0.713)$; healing was significantly slower for wounds of longer baseline duration $(P=0.01)$, and those with peri-wound skin affected by oedema, dermatosclerosis or erythema $(P=0.03)$.

Median (mean) reduction in ulcer area between baseline and 12-week assessment: Group 1: 77\% (58\%); Group 2: $56 \%$ (46\%).

Number of patients rating bandage comfort as 'excellent' out of a total of 38 patients completing this assessment (numbers assessed/group not reported): Group 1: 15; Group 2: 4.

Comparison of costs based on cost/bandage, cost of other disposables (e.g. primary dressings, wadding), and assumption of 30 minutes of nursing/bandage change @ 14 EUR/h.

Cost/patient (EUR): Group 1: EUR 587; Group 2: EUR 1345.

Cost/ulcer healed (EUR): Group 1: EUR 1845; Group 2: EUR 5502.

Number (\%) withdrawals because of patient's request or loss to follow-up: Group 1: 7/44 (16\%); Group 2: $7 / 45(16 \%)$.

Withdrawals due to adverse events: Group 1: 1 withdrawal because of heart and lung problems; Group 2: 1 withdrawal because of pain.

Notes

Patients with bilateral ulceration randomised to receive 1 treatment only, and the limb with the larger total area of ulceration was studied. Wound surface area measured using tracing and computerised planimetry, and ulcers photographed at every clinic visit. 
Ukat 2003 (Continued)

\section{Bias Authors' judgement Support for judgement}

Random sequence genera- Low risk Information from published trial report was unclear:

tion (selection bias)

"This was a prospective randomised controlled comparative study . . . ".

Standard data checks undertaken for the IPD meta-analysis, however, suggested that generation of the random sequence was satisfactory.

$\begin{array}{ll}\begin{array}{l}\text { Allocation concealment } \\ \text { (selection bias) }\end{array} & \text { "Randomisation was performed by opening sealed envelopes containing infor- } \\ & \text { mation about the proposed treatment". }\end{array}$

Incomplete outcome data Low risk (attrition bias)

Information from published trial report: "Patients were analysed according

All outcomes to the treatment received . . . ", "Dropouts were included in the analysis . . " (7 from each Group), however, it was not clear how they were included (may have been last observation carried forward, as the authors stated, "dropouts were included in the analysis as they formed part of the full analysis patient population that is all patients who had a venous leg ulcer, an initial baseline assessment and at least one follow up assessment ... . )".

All randomised patients were included in the patient level analysis.

\begin{tabular}{lll}
\hline $\begin{array}{l}\text { Blinded outcome assess- } \\
\text { ment (healing) }\end{array}$ & High risk & $\begin{array}{l}\text { "The clinician took photographs of the ulcers at every follow up visit ... ". but } \\
\text { no mention of assessment of photographs by anyone else. }\end{array}$ \\
\hline Baseline comparability & Low risk & $\begin{array}{l}\text { Examination of individual patient data indicated satisfactory balance of base- } \\
\text { line variables across groups. }\end{array}$
\end{tabular}

Vowden 2000

Methods $\quad$ RCT in vascular leg ulcer clinic setting, UK.

Participants

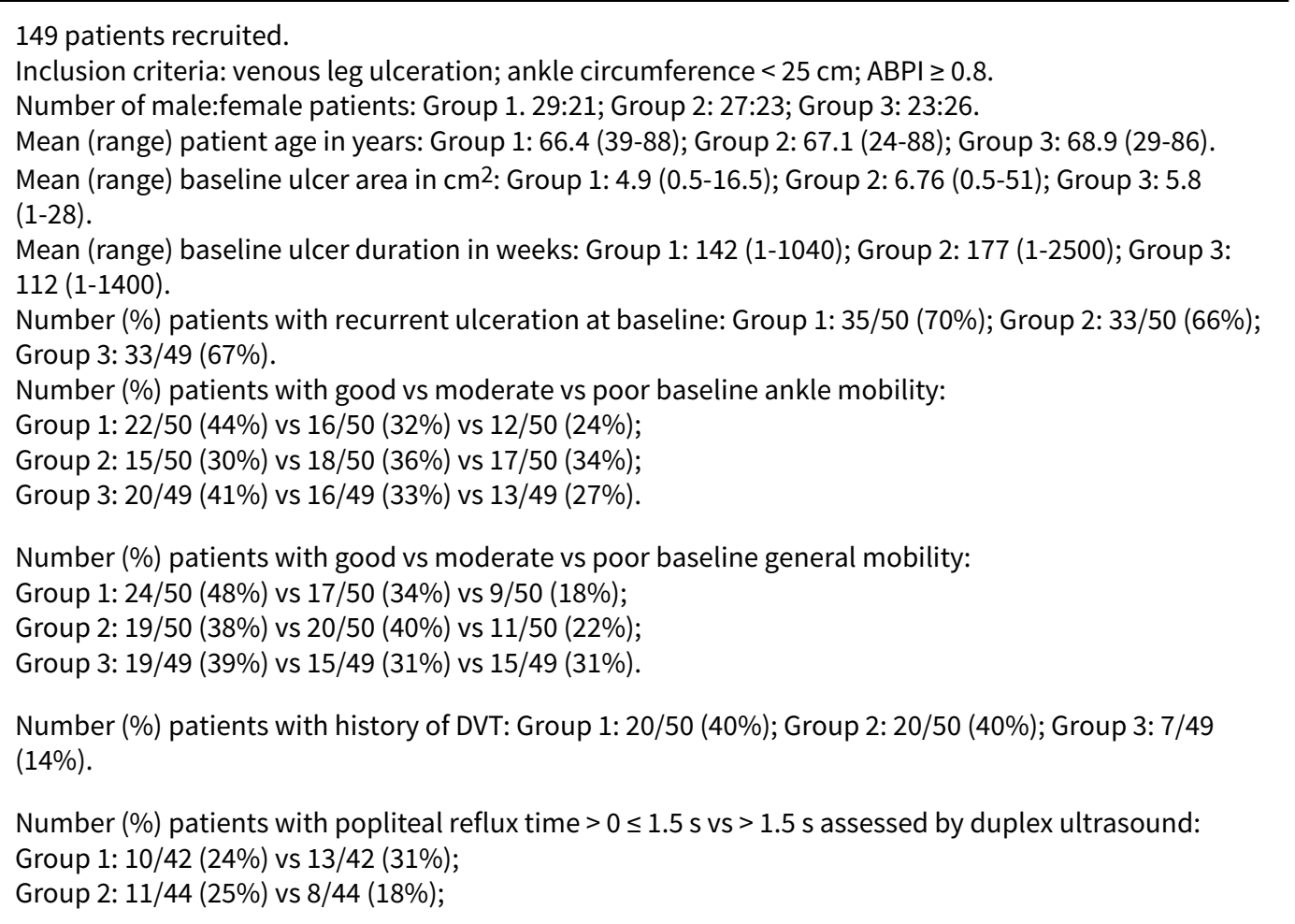


Vowden 2000 (Continued)

Group 3: $10 / 37(27 \%)$ vs $16 / 37$ (43\%).

Interventions All patients received disease-specific information and education (no further details about this) and all received treatment on a weekly basis.

Group 1: original Charing Cross 4LB system consisting of orthopaedic wool (Soffban, Smith \& Nephew), crepe bandage (Smith \& Nephew), elastic bandage (Elset, Seton Scholl) and elastic cohesive bandage (Coban, 3M) ( $n=50)$.

Group 2: modified Charing Cross 4LB system consisting of orthopaedic wool (Soffban, Smith \& Nephew), elastic bandage (K-Lite, Parema), elastic bandage (K-Plus, Parema) and adhesive elastic bandage (Coban, Smith \& Nephew) $(n=50)$.

Group 3: 4LB kit (Robinson Ultra Four) consisting of wound dressing, Sohfast, K-Lite, K-plus and Cohfast $(n=49)$.

At the end of the 20-week study period, patients who had healed received compression stockings, and those who had withdrawn or remained unhealed were treated with the original Charing Cross system.

Outcomes

Patients with complete healing at 12 weeks: Group 1: 60\%; Group 2: 76\%; Group 3: 60\% (chi-squared analysis for comparison between the 3 groups, $P=0.16$ ).

Patients with complete healing at 20 weeks: Group 1: 87\%; Group 2: 84\%; Group 3: 83\% (chi-squared analysis for comparison between the 3 groups, $\mathrm{P}=0.56$ ).

Notes

Estimated cost/bandage system (presumed price year 1999-2000): Group 1: GBP 5.82; Group 2: GBP 4.10; Group 3: GBP 5.83.

There was baseline imbalance for ulcer duration, ulcer area, history of DVT and popliteal reflux.

Few details were provided about wound measurement except to say that ulcers were photographed and mapped.

3 patients withdrew because of non-compliance (breakdown/group not reported).

5 patients were withdrawn because of medical reasons: falling ABPI, skin malignancy on another leg site, medical admission for respiratory disease, cellulitis and death unrelated to treatment (breakdown/group not reported).

Number of patients withdrawn because of potential bandage-related complications, namely persistent skin reddening and discomfort; superficial skin damage: Group 1: 0; 0; Group 2: 2; 1; Group 3: 1 ; 1 . These 5 patients continued with compression bandaging after withdrawal, using an extra padded Charing Cross system, and all healed within 4 weeks of withdrawal.

Assessment of patients' opinion of the bandages by direct questioning during weekly bandage changes, indicated that participants were equally tolerant of all 3 compression systems.

Assessment of staff preference before, during and after the study initially showed a greater preference for the original Charing Cross system, but there was no bandage preference by the end of the 20-week study. This assessment was based on consideration of handling, ease of application, bandage performance over the preceding 7 days and ease of removal.

In the concluding comments, the authors mentioned that care had been provided by expert bandagers.

\section{Risk of bias}

Bias Authors' judgement Support for judgement

Random sequence genera- Unclear risk

No details provided; merely described as a "randomised, controlled study". tion (selection bias) 
Vowden 2000 (Continued)

Allocation concealment (selection bias)

Incomplete outcome data

(attrition bias)

All outcomes
Information from trial author: "randomisation was by sealed envelopes".
High risk
149 people recruited, however, outcomes not presented with denominators, so impossible to judge extent of follow-up.

3 patients withdrew because of non-compliance (breakdown/group not reported).

5 patients were withdrawn because of medical reasons: falling ABPI, skin malignancy on another leg site, medical admission for respiratory disease, cellulitis and death unrelated to treatment (breakdown/group not reported).

Number of patients withdrawn because of potential bandage-related complications, namely persistent skin reddening and discomfort; superficial skin damage: Group 1: 0; 0; Group 2: 2; 1; Group 3: $1 ; 1$. These 5 patients continued with compression bandaging after withdrawal, using an extra padded Charing Cross system, and all healed within 4 weeks of withdrawal.

Contact with the trial authors confirmed that the analysis had been conducted on a per protocol basis; information on denominators not available.

Blinded outcome assess- Unclear risk No details provided.
ment (healing)

ment (healing)

Baseline comparability High risk

Smaller mean ulcer area in Group 1; shorter mean duration in Group 3.

\title{
Wilkinson 1997
}

$\begin{array}{ll}\text { Methods } & \text { RCT (limbs allocated to study groups using a remote randomisation service with numbers generated by } \\ & \text { random number tables, using blocks of } 4 \text { and stratification according to baseline ulcer area: }<9.9 \mathrm{~cm} 2 \\ & \text { and } \geq 10 \mathrm{~cm}^{2} \text { ). Community setting, South Buckinghamshire, UK. }\end{array}$

\section{Participants}

\begin{abstract}
29 patients with 35 ulcerated legs recruited through district and practice nurses.
Inclusion criteria: uncomplicated venous leg ulcer (confirmed by dermatologist) being treated by district or practice nurse.

Exclusion criteria: peripheral vascular disease, cellulitis, $\mathrm{ABPI}<0.8$, contact allergy to latex, ulcer on foot or toes, rheumatoid arthritis, collagen vascular disease, ankle circumference $<18$ or $>25 \mathrm{~cm}$.

Number of limbs belonging to male:female patients: Group 1: 8:9; Group 2: 5:13.

Number of limbs with baseline ulcer area $<9.9 \mathrm{~cm}^{2}$ vs $\geq 10 \mathrm{~cm}^{2}$ : Group 1: 12 vs 5; Group 2: 12 vs 6 .

Mean (range) patient age in years for baseline ulcer area $<9.9 \mathrm{~cm}^{2} \mathrm{vs} \geq 10 \mathrm{~cm}^{2}$ : Group 1: $77(62-86)$ vs 72

(49-92); Group 2: 75 (53-86) vs 76 (49-85).

Mean (range) baseline ulcer area in $\mathrm{cm}^{2}$ : Group 1: 11.2 (0.25-49.6); Group 2: 8.6 (0.25-45.0).

Mean (range) baseline ulcer duration in months for baseline ulcer area $<9.9 \mathrm{~cm}^{2} \mathrm{vs} \geq 10 \mathrm{~cm}^{2}$ : Group 1 :

14.2 (1-48) vs 36.8 (6-60); Group 2: 18.3 (1-48) vs 28.2 (5-60).
\end{abstract} thopaedic wool (Soffban), crepe bandage, elastic bandage (Litepress), and cohesive elastic bandage (Coplus) ( $n=17$ legs).

Group 2: alternative 4LB comprising: knitted viscose primary dressing (Tricotex), elasticated viscose stockinette (Tubifast), lint applied in separate strips horizontally around the leg, elastic bandage (Setopress), and elasticated viscose stockinette (Tubifast) $(n=18$ legs).

All patients: standardised wound cleansing solutions and emollients; bandages changed weekly; patients supplied with class II compression stockings post-healing. 
Wilkinson 1997 (Continued)

Outcomes

Number (\%) limbs with complete healing at 12 weeks: Group 1: 8/17 (47\%); Group 2: 8/18 (44\%) (P= 0.51 , chi-squared test for between-group difference in proportions healed, not healed and withdrawn).

OR $(95 \% \mathrm{Cl})$ estimated by trial authors for healing in Group 1 compared with Group 2: 1.11 (0.24-5.19).

Mean percentage reduction in ulcer area during trial, based on unhealed limbs completing the trial: Group 1: $(n=5) 39 \%$; Group 2: $(n=8) 34 \%(P=0.89$, t-test for between-group difference).

Number (\%) limbs withdrawn from treatment (reasons): Group 1:4/17 (24\%) (developed cellulitis 1, bandage uncomfortable/slipped 1, allergic to bandage 1, bandage too painful 1); Group 2: 2/18 (11\%) (leg painful and possibly infected 1 , bandage too painful 1 ).

Notes

In limbs with more than 1 ulcer, the largest wound was included in the trial. Ulcer area was estimated by diameter product (i.e. maximum length $x$ maximum width of ulcer) every 4 weeks. The trial authors stated that measurements of sub-bandage pressure were not made. Ulcer healing was defined as a "continuous layer of epithelial cells across the ulcer surface". Outcome assessment was non-blind. Nurses were taught to apply the bandages by the research nurse.

\section{Risk of bias}

\begin{tabular}{|c|c|c|}
\hline Bias & Authors' judgement & Support for judgement \\
\hline $\begin{array}{l}\text { Random sequence genera- } \\
\text { tion (selection bias) }\end{array}$ & Low risk & $\begin{array}{l}\text { "... patients' ulcerated legs allocated to one of two groups using numbers } \\
\text { generated by random number tables...". }\end{array}$ \\
\hline $\begin{array}{l}\text { Allocation concealment } \\
\text { (selection bias) }\end{array}$ & Low risk & $\begin{array}{l}\text { "... randomisation was based on random numbers and was calculated in } \\
\text { blocks of four... the nurses ringing for randomisation were unaware of the } \\
\text { block randomisation... ". }\end{array}$ \\
\hline $\begin{array}{l}\text { Incomplete outcome data } \\
\text { (attrition bias) } \\
\text { All outcomes }\end{array}$ & Unclear risk & $\begin{array}{l}\text { Recruited } 29 \text { patients with } 35 \text { limbs and "all } 35 \text { limbs included in the healing } \\
\text { analysis". } 4 \text { limbs were withdrawn from Group } 1 \text { and } 2 \text { from Group 2, there- } \\
\text { fore, not clear how withdrawals included in the analysis (whether assumed un- } \\
\text { healed or whether ascertained healing status). }\end{array}$ \\
\hline $\begin{array}{l}\text { Blinded outcome assess- } \\
\text { ment (healing) }\end{array}$ & High risk & "Not observer blind". \\
\hline Baseline comparability & High risk & $\begin{array}{l}\text { Mean ulcer area greater in Group 1; mean duration data impossible to inter- } \\
\text { pret. }\end{array}$ \\
\hline
\end{tabular}

Zuccarelli 1997

\begin{tabular}{ll}
\hline Methods & Multicentred RCT (5 centres in France). \\
\hline Participants & Recruited 48 patients with venous leg ulcers. \\
& $\begin{array}{l}\text { Inclusion criteria: post-thrombotic superficial venous leg ulcer present for }>4 \text { weeks, showing no signs } \\
\text { of improvement according to clinical examination. }\end{array}$
\end{tabular}

Exclusion criteria: not stated.

Number of male:female patients: Group 1: 7:15; Group 2: 9:17.

Mean \pm SD patient age in years: Group 1: $70 \pm 10$; Group 2: $74 \pm 13$.

Mean \pm SD ulcer duration in months: Group 1: $7 \pm 10$; Group 2: $5 \pm 5$.

Mean \pm SD ulcer surface area in $\mathrm{cm}^{2}$ : Group 1: $4.88 \pm 4.25$; Group 2: $3.38 \pm 3.00$. 
Zuccarelli 1997 (Continued)

Number (\%) patients with ulcer surface area > $4 \mathrm{~cm}^{2}$ : Group 1: 13/22 (59\%); Group 2: 8/26 (31\%).

Interventions Group 1: Elastic bandage (BIFLEX ${ }^{\circledR} 17$, Thuasne, France) applied in spiral configuration with $30 \%$ stretch, designed to provide $20-36 \mathrm{~mm} \mathrm{Hg}$ compression (no report of this being verified) $(n=22)$.

Group 2: SSB $\left(\right.$ Somos $^{\circledast}$, BSN Medical, France) applied as spiral $(n=26)$.

All patients: compression bandages removed at night and primary dressings retained with a gauze bandage. Sclerotherapy, surgery and phlebotropic medication not permitted during the trial. Treatment duration was 2 months.

Outcomes

Mean change in ulcer surface area in $\mathrm{cm}^{2}$ at 2 months: Group 1: -3.1; Group 2: -1.6 (calculated by the review authors from baseline and follow-up ulcer area data provided in the paper).

Number (\%) patients with ulcer surface area decreasing by at least $2 \mathrm{~cm}^{2}$ at 2 months in a subgroup of patients with baseline ulcer area $>4 \mathrm{~cm}^{2}$ : Group 1: 12/13 (92\%); Group 2: 5/8 (62\%), between-group difference reported as not statistically significant by trial authors.

Number (\%) patients with ulcer surface area decreasing by at least $25 \%$ at 2 months in a subgroup of patients with baseline ulcer area $>4 \mathrm{~cm}^{2}$ : Group 1: 12/13 (92\%); Group 2: 5/8 (62\%), between-group difference reported as not statistically significant by trial authors.

Notes Surface area of ulcers assessed at baseline, then every 2 weeks using tracing (no further details).

No information provided about bandager skill or experience.

Trial authors mentioned that they had assessed tolerance of the bandages, but no report of findings.

Original language of report was French; data were extracted with the assistance of a translator.

\section{Risk of bias}

\begin{tabular}{lll}
\hline Bias & Authors' judgement & Support for judgement \\
\hline $\begin{array}{l}\text { Random sequence genera- } \\
\text { tion (selection bias) }\end{array}$ & Unclear risk & " . . a multicentre, randomised clinical trial was conducted ... ". \\
& $\begin{array}{l}\text { Comment: there were no further details of exact methods used to generate the } \\
\text { randomised sequence. }\end{array}$
\end{tabular}

\begin{tabular}{lll}
\hline $\begin{array}{l}\text { Allocation concealment } \\
\text { (selection bias) }\end{array}$ & Unclear risk & No details provided. \\
\hline $\begin{array}{l}\text { Incomplete outcome data } \\
\text { (attrition bias) } \\
\text { All outcomes }\end{array}$ & Unclear risk & $\begin{array}{l}\text { The number of patients included in the assessment of ulcer surface area was } \\
\text { not explicitly stated. }\end{array}$ \\
\hline $\begin{array}{l}\text { Blinded outcome assess- } \\
\text { ment (healing) }\end{array}$ & Unclear risk & No details provided. \\
\hline $\begin{array}{l}\text { Baseline comparability } \\
\text { Unclear risk }\end{array}$ & $\begin{array}{l}\text { Median and ranges not reported for baseline ulcer area and duration, so diffi- } \\
\text { cult to judge (mean values presented). }\end{array}$ \\
\hline
\end{tabular}

In previous versions of this review the study by Scriven 1998 was cited as London et al (1996).

In the previous version of this review Meyer (2000) was referred to (under the section ongoing studies) as Burnand.

In the previous version of this review Moffatt 1999 was cited as McCollum et al (1997). The latter is now a secondary reference of Moffatt 1999.

In the previous version of this review Nelson 2007a was cited as Nelson 1995. The latter is now a secondary reference of Nelson 2007 .

\section{Abbreviations}

$<=$ less than

$\leq=$ less than or equal to 
$>=$ greater/more than

$\geq=$ greater/more than or equal to

$\pm=$ plus or minus

$4 \mathrm{LB}=$ four-layer bandage

$\mathrm{ABPI}=$ ankle brachial pressure index

$\mathrm{BMI}=$ body mass index

CEAP = Clinical severity, Etiology or cause, Anatomy, Pathophysiology (CEAP is a method of classifying venous disease)

$\mathrm{Cl}=$ confidence interval

$\mathrm{CIVIQ}=$ chronic venous insufficiency quality of life questionnaire

DVT = deep vein thrombosis

$\mathrm{GP}=$ general practitioner

$\mathrm{h}=$ hour(s)

$\mathrm{H}_{2} \mathrm{O}_{2}=$ hydrogen peroxide

HIV = human immunodeficiency virus

$\mathrm{HR}=$ hazard ratio

IPD = individual patient data

ITT = intention to treat (analysis)

$M D=$ mean difference

$\mathrm{NB}=$ please note

$\mathrm{RCT}=$ randomised controlled trial

$\mathrm{s}=\operatorname{second}(\mathrm{s})$

$\mathrm{SD}=$ standard deviation

$\mathrm{SE}=$ standard error

SEM = standard error of the mean

SF-36 = Short Form 36

$\mathrm{SSB}=$ short stretch bandage

vs $=$ versus

Characteristics of excluded studies [ordered by study ID]

\begin{tabular}{|c|c|}
\hline Study & Reason for exclusion \\
\hline Alvarez 2005 & Not randomised (confirmed through correspondence with the first author). \\
\hline Baccaglini 1998 & Not randomised. \\
\hline Blair 1988 & Primarily a dressings trial; comparison between bandages not randomised. \\
\hline Brizzio 2006 & Not randomised. \\
\hline Cameron 1996 & Historical control, therefore not randomised. \\
\hline Cherry 1990 & Healing not measured as an outcome. \\
\hline Falanga 1998 & $\begin{array}{l}\text { Treatment groups differed systematically other than in terms of the compression systems used. } \\
\text { One group received a topical application of human skin equivalent plus a nonadherent primary } \\
\text { dressing and an elastic bandage; the other received a nonadherent primary dressing, paste ban- } \\
\text { dage and elastic bandage. }\end{array}$ \\
\hline Fuessl 2009 & Brief commentary on meta-analysis (O'Meara 2009). \\
\hline Hamel-Desnos 2010 & Interventions designed to treat varicose veins, not ulceration. \\
\hline Heinen 2010 & $\begin{array}{l}\text { Did not evaluate compression (evaluated an intervention to promote patient concordance with } \\
\text { compression). }\end{array}$ \\
\hline Hjerppe 2010 & Prognostic study, not RCT. \\
\hline
\end{tabular}




\begin{tabular}{|c|c|}
\hline Study & Reason for exclusion \\
\hline Horakova 1994 & Not randomised. \\
\hline Ivanovic 2011 & Not an RCT, a brief review of the previous version of this Cochrane review (confirmed by translator). \\
\hline Jull 2009 & Not a randomised comparison. \\
\hline Jünger 2006 & Patients did not have ulceration; primary outcome was skin condition. \\
\hline Kucharzewski 2003 & Not randomised. \\
\hline Kuznetsov 2009 & $\begin{array}{l}\text { Both groups had same method of compression, comparison was of dressings (confirmed by trans- } \\
\text { lator). }\end{array}$ \\
\hline Lee 2009 & Not an RCT; overview and study of bandage pressures in healthy volunteers. \\
\hline Luo 2009 & Not an RCT (a case series - confirmed by translator). \\
\hline Marston 1999 & Not randomised. \\
\hline Nissinen-Paatsamala 1995 & Not randomised. \\
\hline Northeast 1990 & Treatment effect confounded by use of steroids in one treatment group but not the other. \\
\hline Olofsson 1996 & $\begin{array}{l}\text { Treatment groups differed systematically other than in terms of compression systems used (one } \\
\text { group treated by a surgeon and the other by dermatologists). In addition, several different types of } \\
\text { compression were used within each group, meaning that the relative effectiveness of each system } \\
\text { would be difficult to estimate. }\end{array}$ \\
\hline Partsch 2008a & Patients with leg ulceration were excluded. \\
\hline Robson 2004 & Trial of topical applications; all patients received same type of compression. \\
\hline Russo 1999 & Have abstract only; randomisation not mentioned. \\
\hline Sabolinski 1995 & Both groups had compression, comparison was of dressings. \\
\hline Scriven 2000 & Case series that primarily assessed sub-bandage pressures. \\
\hline Serra 2010 & Dressings trial; all patients received same type of compression (confirmed by translator). \\
\hline Sikes 1985 & Not randomised. \\
\hline Sironi 1994 & $\begin{array}{l}\text { Comparison of different protocols of delivering dressings and topical agents; patients in both study } \\
\text { groups received the same type of compression. }\end{array}$ \\
\hline Smith Strom 2006 & Dressings trial; all patients received same type of compression. \\
\hline Szewczyk 2009 & Quasi-randomised. \\
\hline Torra i Bou 2003 & Not randomised (uncontrolled before-after study). \\
\hline Van Laere 2010 & Quasi-randomised (author provided additional information). \\
\hline Vowden 2001 & Non-comparative study. \\
\hline
\end{tabular}




\begin{tabular}{ll}
\hline Study & Reason for exclusion \\
\hline Walker 1996 & $\begin{array}{l}\text { Have abstract only; no objective wound healing data presented and attempts to obtain further in- } \\
\text { formation from trial authors were unsuccessful. }\end{array}$ \\
\hline Zamboni 2004 & $\begin{array}{l}\text { Comparison is venous reflux surgery versus compression but both study groups received the same } \\
\text { type of compression system. }\end{array}$ \\
\hline
\end{tabular}

\section{Abbreviations}

$\mathrm{RCT}=$ randomised controlled trial

Characteristics of studies awaiting assessment [ordered by study ID]

Bertaux 2010

Methods Comparative study, unclear whether RCT.

\begin{tabular}{ll}
\hline Participants & 60 patients with venous leg ulcers \\
\hline Interventions & SSB versus compression stockings \\
\hline Outcomes & Proportion of patients with complete healing \\
\hline Notes & Only abstract currently available; awaiting full report.
\end{tabular}

\section{Harrison 2011}

\begin{tabular}{ll}
\hline Methods & RCT. \\
\hline Participants & 424 patients with venous leg ulcers. \\
\hline Interventions & 4LB vs SSB. \\
\hline Outcomes & Healing, recurrence, adverse events. \\
\hline Notes & Yet to contact trial authors regarding data retrieval. \\
\hline
\end{tabular}

Moffatt 2003b

\begin{tabular}{ll}
\hline Methods & RCT. \\
\hline Participants & 300 patients with venous leg ulcers. \\
\hline Interventions & 4LB vs compression with 2 components (Proguide, Smith \& Nephew). \\
\hline Outcomes & No data. \\
\hline Notes & Only abstract currently available; awaiting full report. \\
\hline
\end{tabular}


Mosti 2010

\begin{tabular}{ll}
\hline Methods & RCT. \\
\hline Participants & Patients with non-infected leg ulcers eligible to receive compression. \\
\hline Interventions & Different short-stretch compression systems and different dressings. \\
\hline Outcomes & Healing mentioned, but no data provided. \\
\hline Notes & Only abstract currently available; awaiting full report. \\
\hline
\end{tabular}

Mosti 2011

\begin{tabular}{ll}
\hline Methods & RCT. \\
\hline Participants & 100 patients with venous leg ulcers. \\
\hline Interventions & Paste bandage vs compression bandage with 2 components. \\
\hline Outcomes & Proportion of patients with complete healing. \\
\hline Notes & Seeking clarification from trial authors regarding components of compression. \\
\hline
\end{tabular}

Taradaj 2011

\begin{tabular}{ll}
\hline Methods & Unclear whether an RCT. \\
\hline Participants & 305 patients with venous leg ulcers. \\
\hline Interventions & 10 different treatment arms, including comparison of compression vs no compression. \\
\hline Outcomes & Proportion of patients with complete healing. \\
\hline Notes & Unclear whether really randomised - seeking clarification from trial authors. \\
\hline
\end{tabular}

Wong 2012

\begin{tabular}{ll}
\hline Methods & RCT. \\
\hline Participants & 321 patients with venous leg ulcers. \\
\hline Interventions & 4LB vs SSB vs no compression (dressing only). \\
\hline Outcomes & Proportion of patients with complete healing. \\
\hline Notes & Yet to contact trial authors regarding data retrieval. \\
\hline
\end{tabular}

\section{Abbreviations}

$4 \mathrm{LB}=$ four-layer bandage

$\mathrm{RCT}=$ randomised controlled trial

$\mathrm{SSB}=$ short stretch bandage 
vs $=$ versus

Characteristics of ongoing studies [ordered by study ID]

Dumville 2009

\begin{tabular}{ll}
\hline Trial name or title & $\begin{array}{l}\text { VenUS IV: Compression hosiery versus compression bandaging in the treatment of venous leg ul- } \\
\text { cers. }\end{array}$ \\
\hline Methods & RCT. \\
\hline Participants & Patients with venous leg ulceration confirmed by ABPI $\geq 0.8$. \\
\hline Interventions & Compression hosiery versus 4LB. \\
\hline Outcomes & $\begin{array}{l}\text { Time to healing, cost of treatment, quality of life, patient concordance with treatment, recurrence } \\
\text { of ulceration. }\end{array}$ \\
\hline Starting date & May 2009. \\
\hline Contact information & Dr Jo Dumville (jo.dumville@york.ac.uk) \\
\hline Notes &
\end{tabular}

\section{Matos de Abreu 2011}

\begin{tabular}{ll}
\hline Trial name or title & None provided. \\
\hline Methods & RCT. \\
\hline Participants & Patients with venous ulcers. \\
\hline Interventions & Unna's boot versus elastic bandages. \\
\hline Outcomes & Not specified. \\
\hline Starting date & Not specified. \\
\hline Contact information & Alcione Matos de Abreu (alci_abreu@yahoo.com.br) \\
\hline Notes & $\begin{array}{l}\text { This is a short paper, published in Portuguese. Information from this and the English language ab- } \\
\text { stract indicate a clinical trial which is about to start or is in progress. }\end{array}$ \\
\hline
\end{tabular}

\section{Weller 2010}

\begin{tabular}{ll}
\hline Trial name or title & None provided. \\
\hline Methods & Protocol for an RCT. \\
\hline Participants & Patients with venous leg ulceration confirmed by clinical assessment. \\
\hline Interventions & Tubular compression bandage versus SSB. \\
\hline Outcomes & Proportion of patients with complete healing.
\end{tabular}


Weller 2010 (Continued)

Contact information

\section{Abbreviations}

$\geq=$ greater/more than or equal to

$4 \mathrm{LB}=$ four-layer bandage

$A B P I=$ ankle brachial pressure index

$\mathrm{RCT}=$ randomised controlled trial

$\mathrm{SSB}=$ short-stretch bandage

\section{DATA AND ANALYSES}

Comparison 1. Compression vs no compression (primary dressing only)

\begin{tabular}{llllll}
\hline Outcome or subgroup title & $\begin{array}{l}\text { No. of } \\
\text { studies }\end{array}$ & $\begin{array}{l}\text { No. of par- } \\
\text { ticipants }\end{array}$ & Statistical method & Effect size \\
\hline 1 Ulcers completely healed at 6 months & 1 & 87 & Risk Ratio (M-H, Fixed, 95\% Cl) & $1.50[0.90,2.50]$ \\
\hline
\end{tabular}

Analysis 1.1. Comparison 1 Compression vs no compression (primary dressing only), Outcome 1 Ulcers completely healed at 6 months.

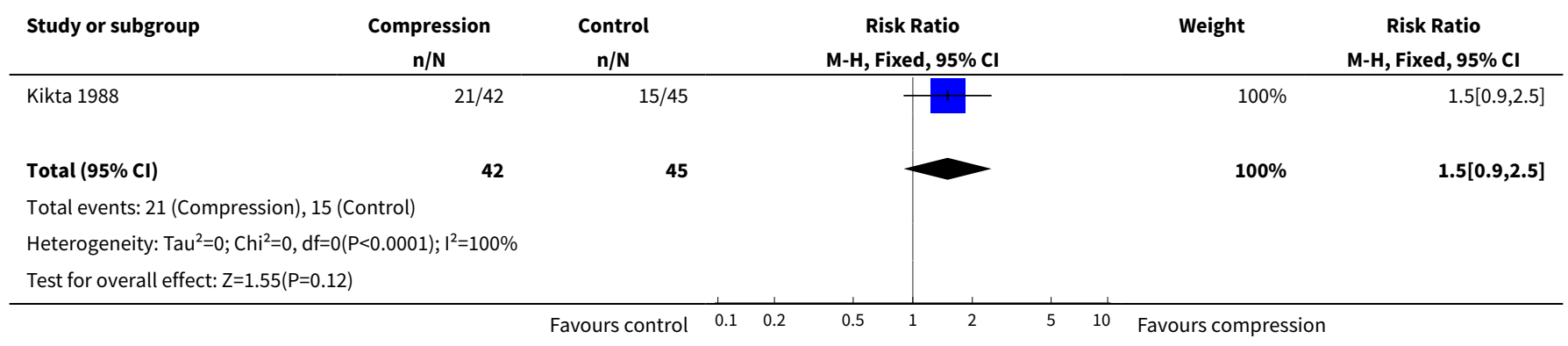

\section{Comparison 2. Compression vs no compression (non-compressive bandage)}

\begin{tabular}{llllll}
\hline Outcome or subgroup title & $\begin{array}{l}\text { No. of } \\
\text { studies }\end{array}$ & $\begin{array}{l}\text { No. of par- } \\
\text { ticipants }\end{array}$ & Statistical method & Effect size \\
\hline 1 Patients with complete healing at 1 year & 1 & 36 & Risk Ratio (M-H, Fixed, 95\% Cl) & $2.30[1.29,4.10]$ \\
\hline
\end{tabular}


Analysis 2.1. Comparison 2 Compression vs no compression (noncompressive bandage), Outcome 1 Patients with complete healing at 1 year.

\begin{tabular}{|c|c|c|c|c|c|}
\hline Study or subgroup & $\begin{array}{c}\text { Compression } \\
n / N\end{array}$ & $\begin{array}{c}\text { Control } \\
\mathrm{n} / \mathrm{N}\end{array}$ & $\begin{array}{c}\text { Risk Ratio } \\
\text { M-H, Fixed, } 95 \% \mathrm{Cl}\end{array}$ & Weight & $\begin{array}{c}\text { Risk Ratio } \\
\text { M-H, Fixed, } 95 \% \mathrm{Cl}\end{array}$ \\
\hline Rubin 1990 & $18 / 19$ & $7 / 17$ & 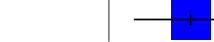 & $100 \%$ & $2.3[1.29,4.1]$ \\
\hline Total $(95 \% \mathrm{Cl})$ & 19 & 17 & & $100 \%$ & $2.3[1.29,4.1]$ \\
\hline \multicolumn{6}{|c|}{ Total events: 18 (Compression), 7 (Control) } \\
\hline \multicolumn{6}{|c|}{ Heterogeneity: Not applicable } \\
\hline Test for overall effec & & & & & \\
\hline
\end{tabular}

Comparison 3. Compression vs no compression (usual treatment)

\begin{tabular}{llllll}
\hline Outcome or subgroup title & $\begin{array}{l}\text { No. of } \\
\text { studies }\end{array}$ & $\begin{array}{l}\text { No. of } \\
\text { partici- } \\
\text { pants }\end{array}$ & Statistical method & Effect size \\
\hline $\begin{array}{l}1 \text { Patients with complete healing at 3 } \\
\text { months }\end{array}$ & 1 & 36 & Risk Ratio (M-H, Fixed, 95\% Cl) & $4.0[1.35,11.82]$ \\
\hline $\begin{array}{l}\text { 2 Patients with complete healing at 1 year } \\
\text { 3 Patients with recurrence during 1 year fol- } \\
\text { low-up }\end{array}$ & 1 & 233 & Risk Ratio (M-H, Fixed, 95\% Cl) & $1.18[0.96,1.47]$ \\
\hline
\end{tabular}

Analysis 3.1. Comparison 3 Compression vs no compression (usual treatment), Outcome 1 Patients with complete healing at 3 months.

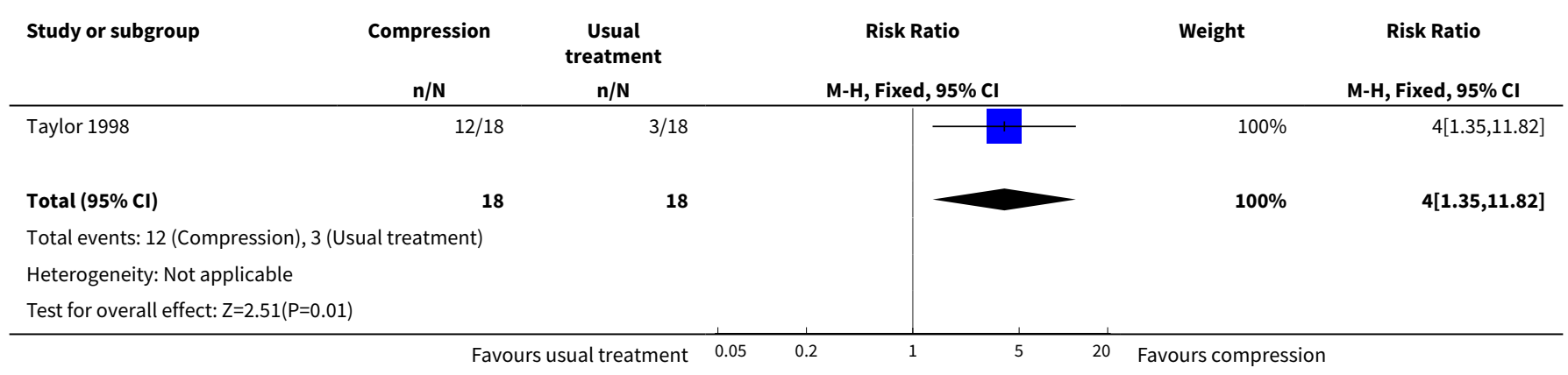

Analysis 3.2. Comparison 3 Compression vs no compression (usual treatment), Outcome 2 Patients with complete healing at 1 year.

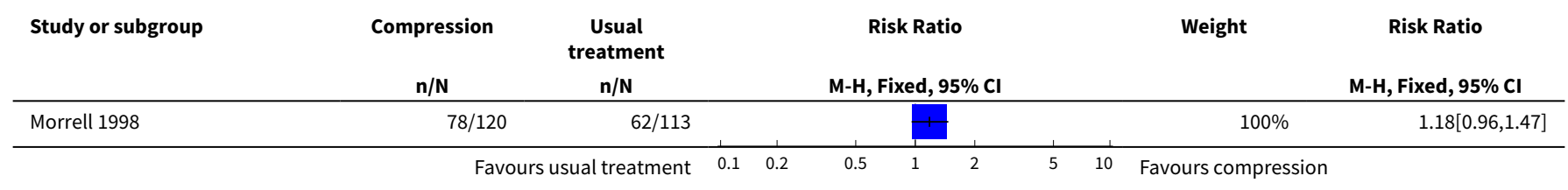




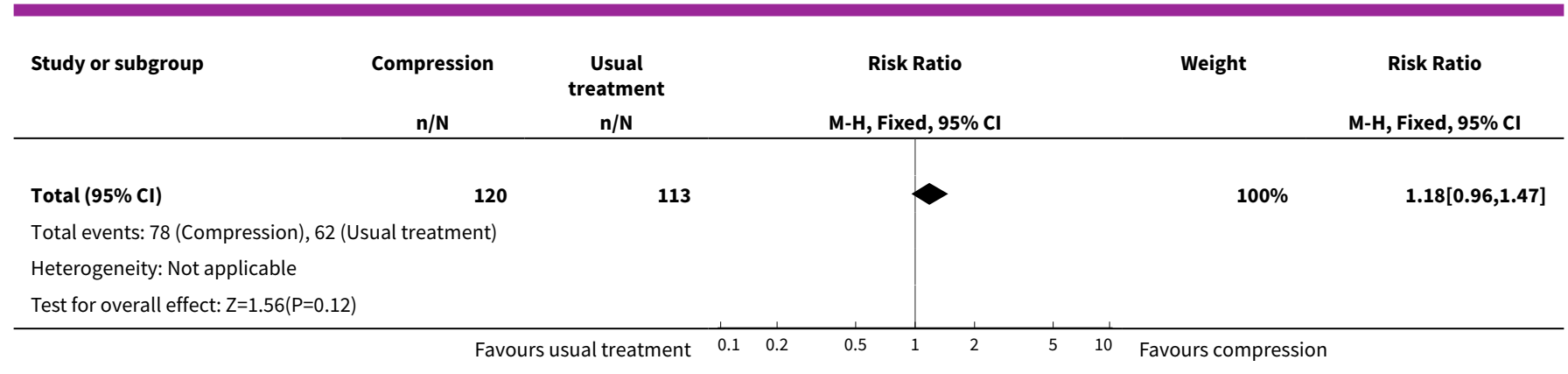

Analysis 3.3. Comparison 3 Compression vs no compression (usual treatment), Outcome 3 Patients with recurrence during 1 year follow-up.

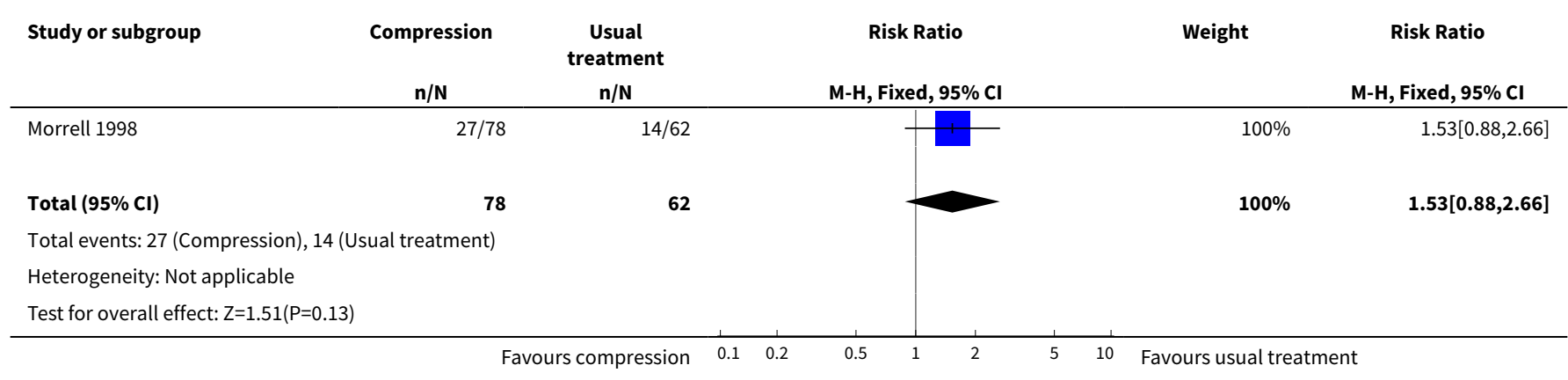

Comparison 4. Single-component compression (elastic bandage) vs single-component compression (paste bandage)

\begin{tabular}{lllll}
\hline Outcome or subgroup title & $\begin{array}{l}\text { No. of } \\
\text { studies }\end{array}$ & $\begin{array}{l}\text { No. of } \\
\text { partici- } \\
\text { pants }\end{array}$ & Statistical method & Effect size \\
\hline 1 Patients with complete healing at 3 months & 1 & Risk Ratio (M-H, Fixed, 95\% Cl) & $\begin{array}{l}\text { Totals not select- } \\
\text { ed }\end{array}$ \\
\hline $\begin{array}{l}2 \text { Percentage change during trial relative to } \\
\text { baseline ulcer area }\end{array}$ & 1 & Mean Difference (IV, Fixed, 95\% Cl) & $\begin{array}{l}\text { Totals not select- } \\
\text { ed }\end{array}$ \\
\hline $\begin{array}{l}3 \text { Healing rate (cm squared per week adjusted } \\
\text { for baseline ulcer perimeter) }\end{array}$ & 1 & Mean Difference (IV, Fixed, 95\% Cl) & Totals not select- \\
\hline
\end{tabular}

Analysis 4.1. Comparison 4 Single-component compression (elastic bandage) vs singlecomponent compression (paste bandage), Outcome 1 Patients with complete healing at 3 months.

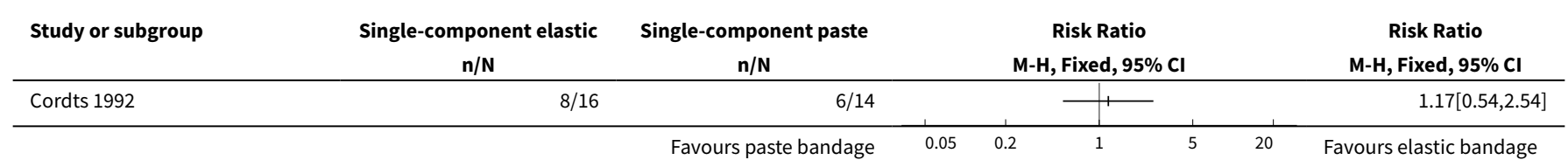


Analysis 4.2. Comparison 4 Single-component compression (elastic bandage) vs single-component compression (paste bandage), Outcome 2 Percentage change during trial relative to baseline ulcer area.

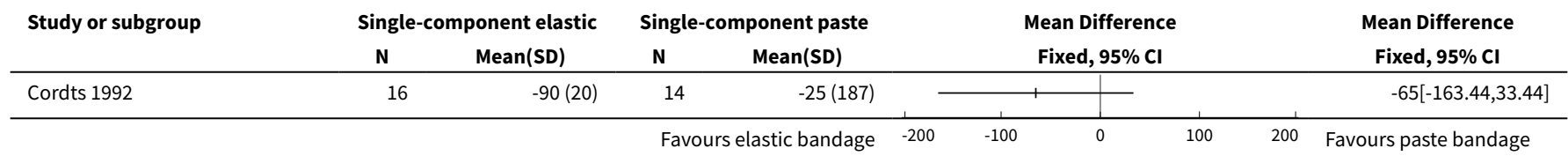

Analysis 4.3. Comparison 4 Single-component compression (elastic bandage) vs single-component compression (paste bandage), Outcome 3 Healing rate ( $\mathrm{cm}$ squared per week adjusted for baseline ulcer perimeter).

\begin{tabular}{|c|c|c|c|c|c|c|c|c|c|}
\hline \multirow[t]{2}{*}{ Study or subgroup } & \multicolumn{2}{|c|}{ Single-component elastic } & \multicolumn{2}{|c|}{ Single-component paste } & & \multirow{2}{*}{\multicolumn{2}{|c|}{$\begin{array}{c}\text { Mean Difference } \\
\text { Fixed, } 95 \% \mathrm{CI}\end{array}$}} & & \multirow{2}{*}{$\begin{array}{c}\text { Mean Difference } \\
\text { Fixed, } 95 \% \mathrm{Cl} \\
\end{array}$} \\
\hline & $\mathbf{N}$ & $\operatorname{Mean}(\mathrm{SD})$ & $\mathbf{N}$ & $\operatorname{Mean}(S D)$ & & & & & \\
\hline Cordts 1992 & 16 & $0(0)$ & 14 & $0(0.1)$ & & & & & $0.03[-0.01,0.07]$ \\
\hline
\end{tabular}

Comparison 5. Single-component compression vs multi-component compression (excluding paste bandages)

\begin{tabular}{lllll}
\hline Outcome or subgroup title & $\begin{array}{l}\text { No. of } \\
\text { studies }\end{array}$ & $\begin{array}{l}\text { No. of } \\
\text { partici- } \\
\text { pants }\end{array}$ & Statistical method & Effect size \\
\hline 1 Complete healing during the trial period & 4 & Risk Ratio (M-H, Random, 95\% Cl) & $\begin{array}{l}\text { Totals not select- } \\
\text { ed }\end{array}$ \\
\hline $\begin{array}{l}\text { 2 Percentage change during trial relative to } \\
\text { baseline ulcer area }\end{array}$ & 1 & $\begin{array}{l}\text { Mean Difference (IV, Fixed, 95\% } \\
\text { Cl) }\end{array}$ & $\begin{array}{l}\text { Totals not select- } \\
\text { ed }\end{array}$ \\
\hline $\begin{array}{l}\text { 3 Complete healing during the trial period (par- } \\
\text { ticipants with simple VLU only) }\end{array}$ & 1 & Risk Ratio (M-H, Fixed, 95\% Cl) & Totals not select- \\
\hline
\end{tabular}

Analysis 5.1. Comparison 5 Single-component compression vs multi-component compression (excluding paste bandages), Outcome 1 Complete healing during the trial period.

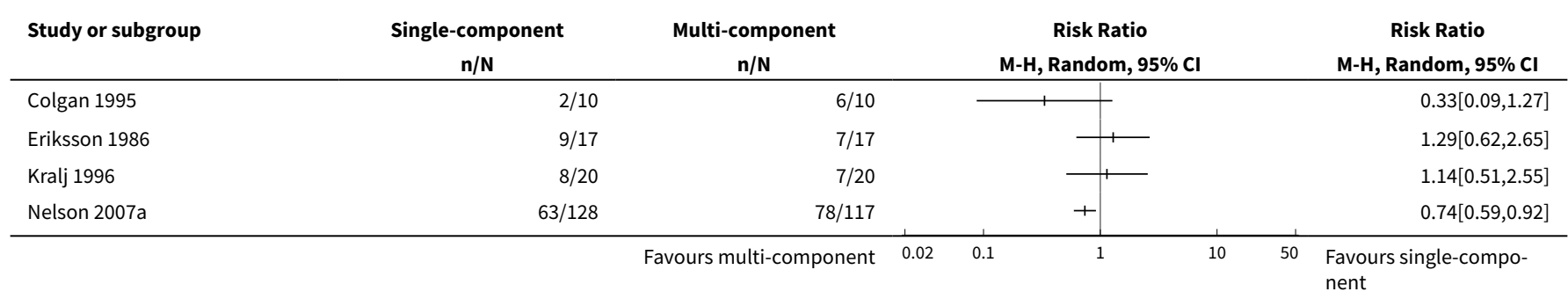


Analysis 5.2. Comparison 5 Single-component compression vs multi-component compression

(excluding paste bandages), Outcome 2 Percentage change during trial relative to baseline ulcer area.

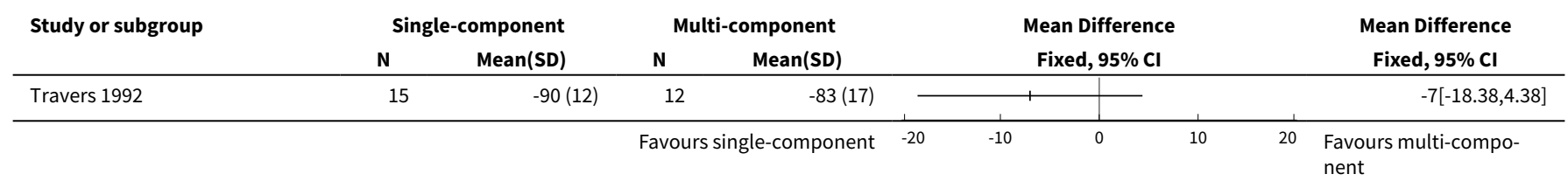

Analysis 5.3. Comparison 5 Single-component compression vs multi-component compression (excluding paste bandages), Outcome 3 Complete healing during the trial period (participants with simple VLU only).

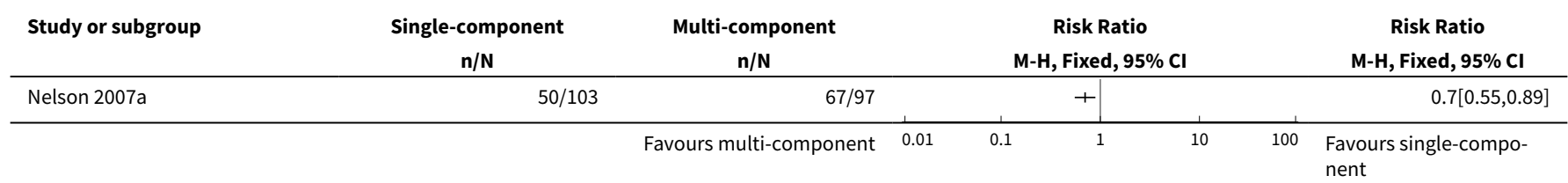

Comparison 6. Single-component compression vs multi-component compression (paste bandage)

\begin{tabular}{lllll}
\hline Outcome or subgroup title & $\begin{array}{l}\text { No. of } \\
\text { studies }\end{array}$ & $\begin{array}{l}\text { No. of par- } \\
\text { ticipants }\end{array}$ & Statistical method & Effect size \\
\hline 1 Complete healing during the trial period & 1 & Risk Ratio (M-H, Fixed, 95\% Cl) & Totals not selected \\
\hline
\end{tabular}

Analysis 6.1. Comparison 6 Single-component compression vs multi-component compression (paste bandage), Outcome 1 Complete healing during the trial period.

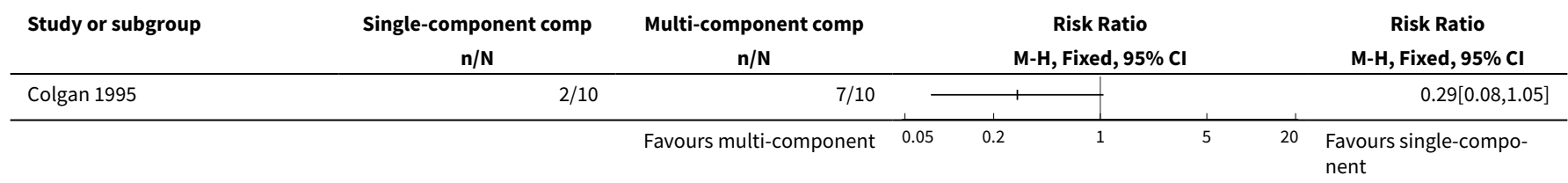

Comparison 7. Two-component (outer elastic) vs two-component (outer inelastic)

\begin{tabular}{lllll}
\hline Outcome or subgroup title & $\begin{array}{l}\text { No. of } \\
\text { studies }\end{array}$ & $\begin{array}{l}\text { No. of } \\
\text { partici- } \\
\text { pants }\end{array}$ & Statistical method & Effect size \\
\hline $\begin{array}{l}1 \text { Patients with complete healing at 3-6 } \\
\text { months }\end{array}$ & 2 & 95 & Risk Ratio (M-H, Fixed, 95\% Cl) & $1.23[0.67,2.25]$ \\
\hline $\begin{array}{l}2 \text { Patients with complete healing at 1 } \\
\text { month }\end{array}$ & 1 & 43 & Risk Ratio (M-H, Fixed, 95\% Cl) & $3.48[0.42,28.63]$ \\
\hline \begin{tabular}{l} 
3 Patients with complete healing at 1 year \\
\hline
\end{tabular} & 1 & 43 & Risk Ratio (M-H, Fixed, 95\% Cl) & $3.48[1.14,10.60]$ \\
\hline
\end{tabular}


Analysis 7.1. Comparison 7 Two-component (outer elastic) vs two-component (outer inelastic), Outcome 1 Patients with complete healing at 3-6 months.

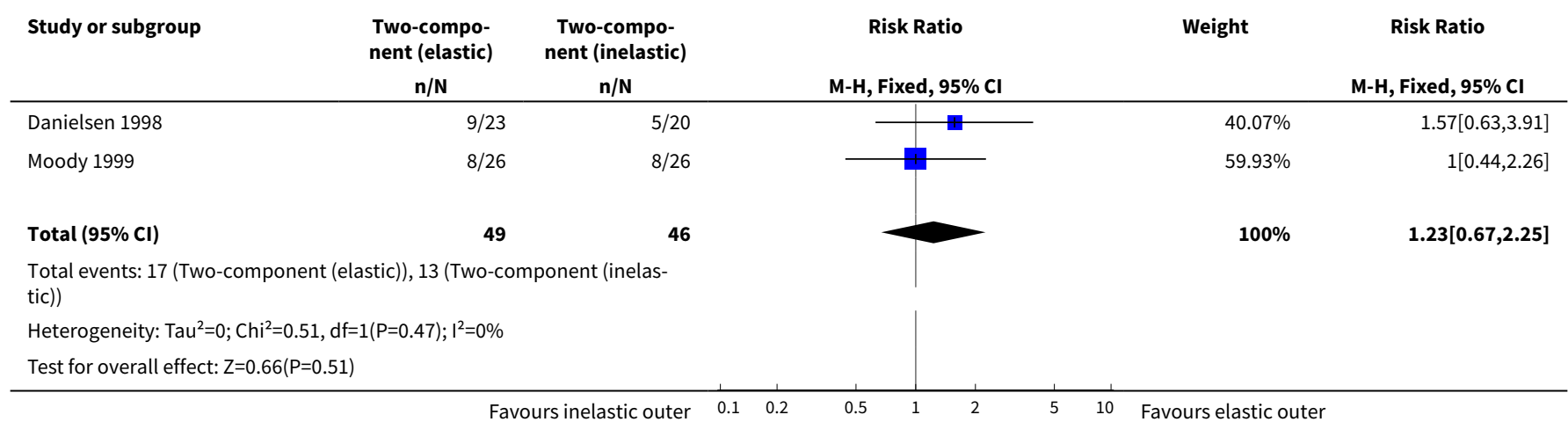

Analysis 7.2. Comparison 7 Two-component (outer elastic) vs two-component (outer inelastic), Outcome 2 Patients with complete healing at 1 month.

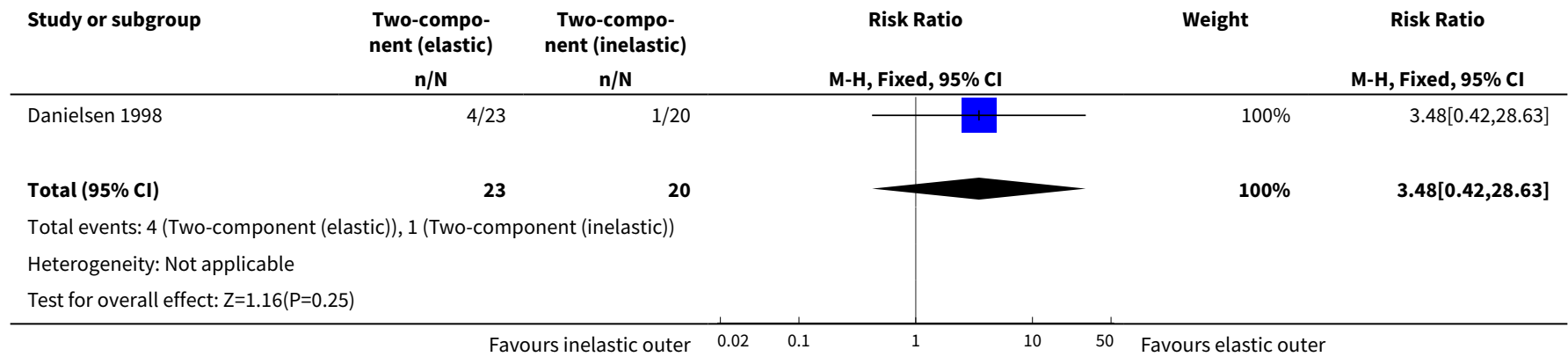

Analysis 7.3. Comparison 7 Two-component (outer elastic) vs two-component (outer inelastic), Outcome 3 Patients with complete healing at 1 year.

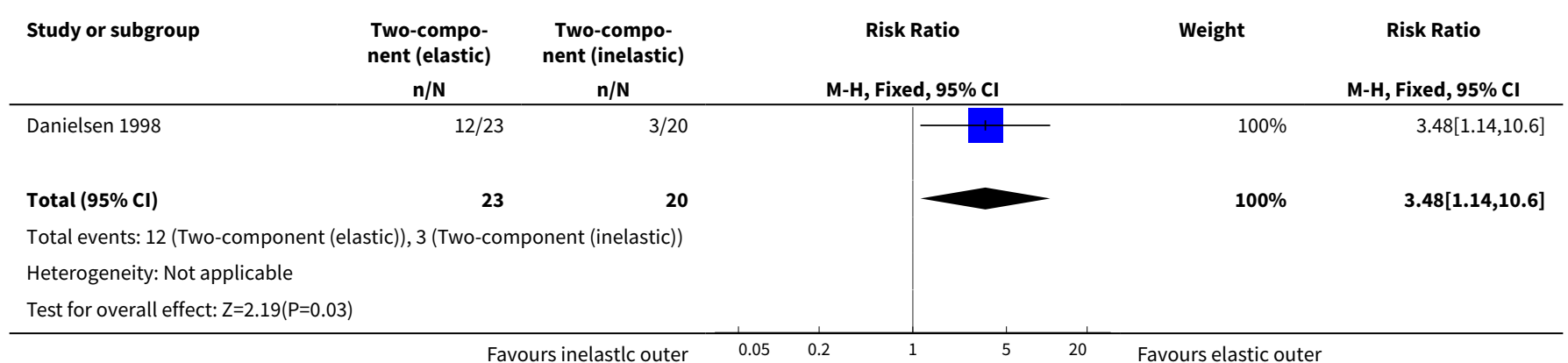


Comparison 8. Two-component system vs four-layer bandage (4LB)

\begin{tabular}{|c|c|c|c|c|}
\hline Outcome or subgroup title & $\begin{array}{l}\text { No. of } \\
\text { studies }\end{array}$ & $\begin{array}{l}\text { No. of } \\
\text { partici- } \\
\text { pants }\end{array}$ & Statistical method & Effect size \\
\hline 1 Patients with complete healing at 1 month & 1 & & Risk Ratio (M-H, Fixed, 95\% Cl) & $\begin{array}{l}\text { Totals not se- } \\
\text { lected }\end{array}$ \\
\hline 2 Patients with complete healing at 3 months & 3 & 170 & Risk Ratio (M-H, Fixed, 95\% Cl) & $0.83[0.66,1.05]$ \\
\hline $\begin{array}{l}3 \text { Patients with complete healing at } 6 \text { months up to } \\
\text { point of withdrawal from randomised treatment }\end{array}$ & 1 & 109 & Risk Ratio (M-H, Fixed, 95\% Cl) & $0.56[0.41,0.77]$ \\
\hline $\begin{array}{l}4 \text { Patients with complete healing at } 6 \text { months includ- } \\
\text { ing withdrawals from randomised treatment }\end{array}$ & 1 & 109 & Risk Ratio (M-H, Fixed, 95\% Cl) & $0.88[0.73,1.05]$ \\
\hline
\end{tabular}

Analysis 8.1. Comparison 8 Two-component system vs four-layer bandage (4LB), Outcome 1 Patients with complete healing at 1 month.

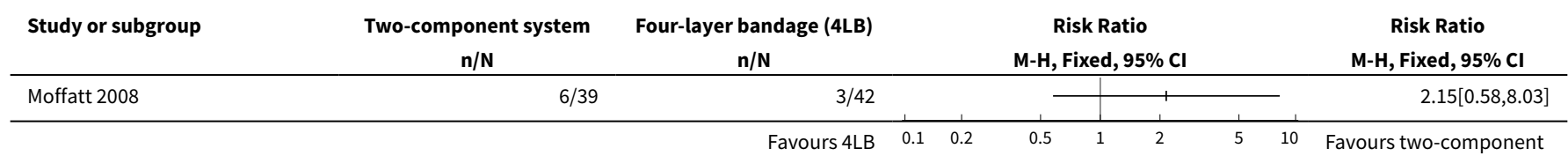

Analysis 8.2. Comparison 8 Two-component system vs four-layer bandage (4LB), Outcome 2 Patients with complete healing at 3 months.

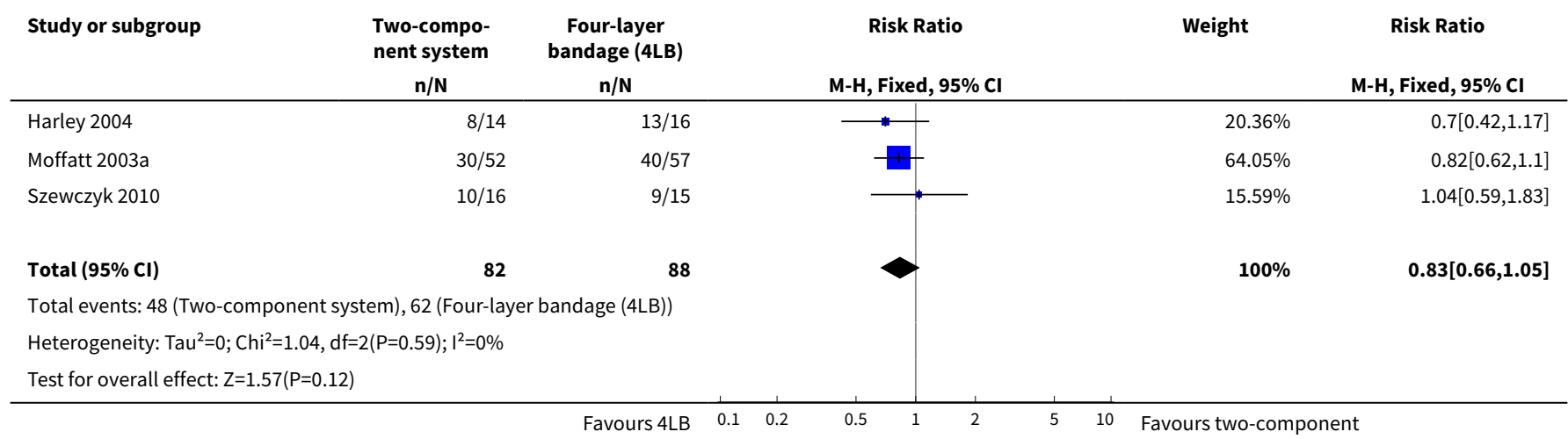

Analysis 8.3. Comparison 8 Two-component system vs four-layer bandage (4LB), Outcome 3 Patients with complete healing at 6 months up to point of withdrawal from randomised treatment.

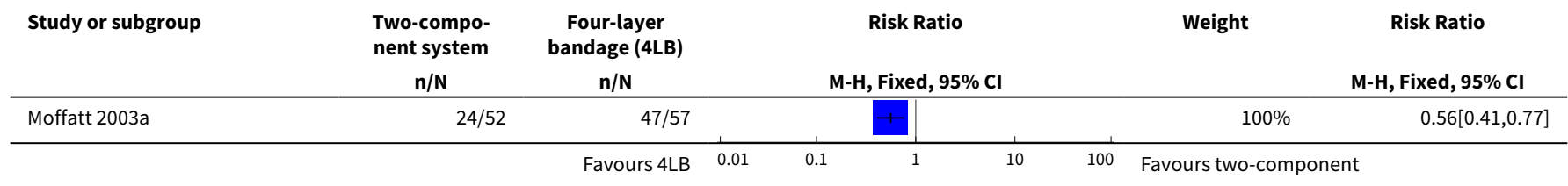




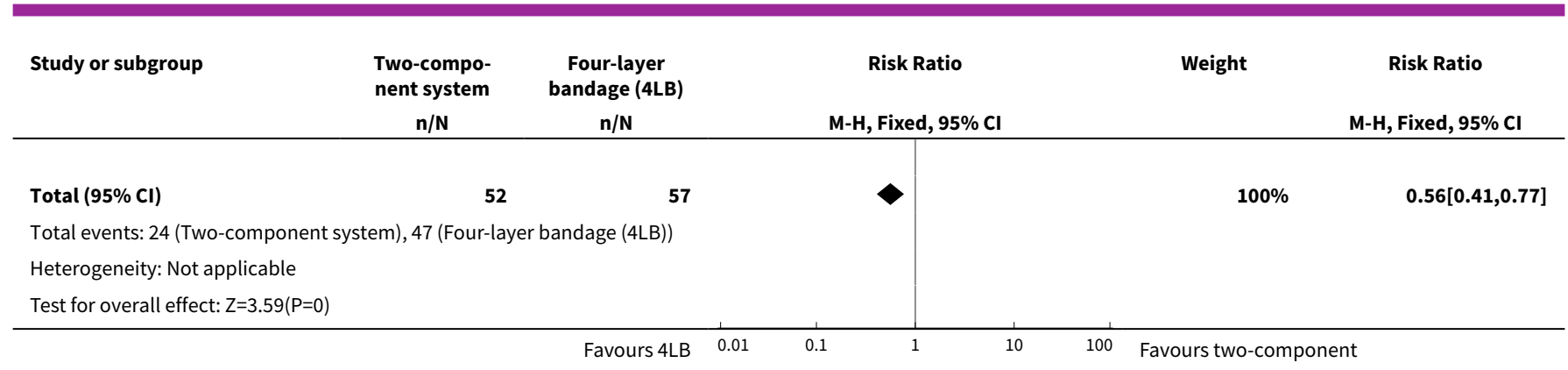

Analysis 8.4. Comparison 8 Two-component system vs four-layer bandage (4LB), Outcome 4 Patients with complete healing at 6 months including withdrawals from randomised treatment.

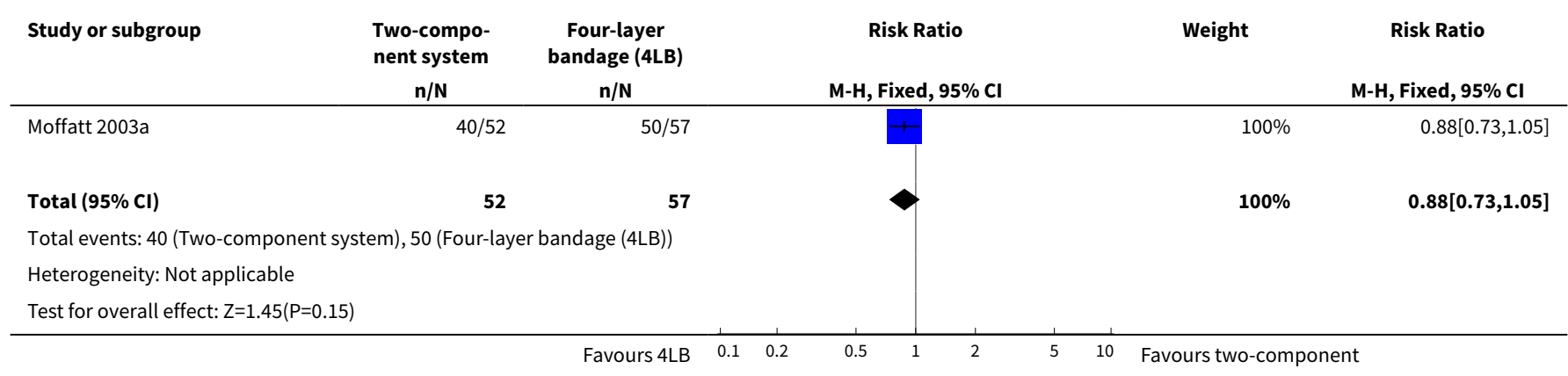

Comparison 9. 3 components including elastic bandage vs 3 components including inelastic bandage

\begin{tabular}{lllll}
\hline Outcome or subgroup title & $\begin{array}{l}\text { No. of } \\
\text { studies }\end{array}$ & $\begin{array}{l}\text { No. of } \\
\text { partici- } \\
\text { pants }\end{array}$ & Statistical method & Effect size \\
\hline $\begin{array}{l}1 \text { Patients/limbs with complete healing } \\
\text { during trial }\end{array}$ & 3 & & Risk Ratio (M-H, Fixed, 95\% Cl) & Subtotals only \\
\hline 1.1 Complete healing at 3-4 months & 2 & 171 & Risk Ratio (M-H, Fixed, 95\% Cl) & $1.83[1.26,2.67]$ \\
\hline 1.2 Complete healing at 6 months & 1 & 112 & Risk Ratio (M-H, Fixed, 95\% Cl) & $0.94[0.69,1.27]$ \\
\hline
\end{tabular}

Analysis 9.1. Comparison 93 components including elastic bandage vs 3 components including inelastic bandage, Outcome 1 Patients/limbs with complete healing during trial.

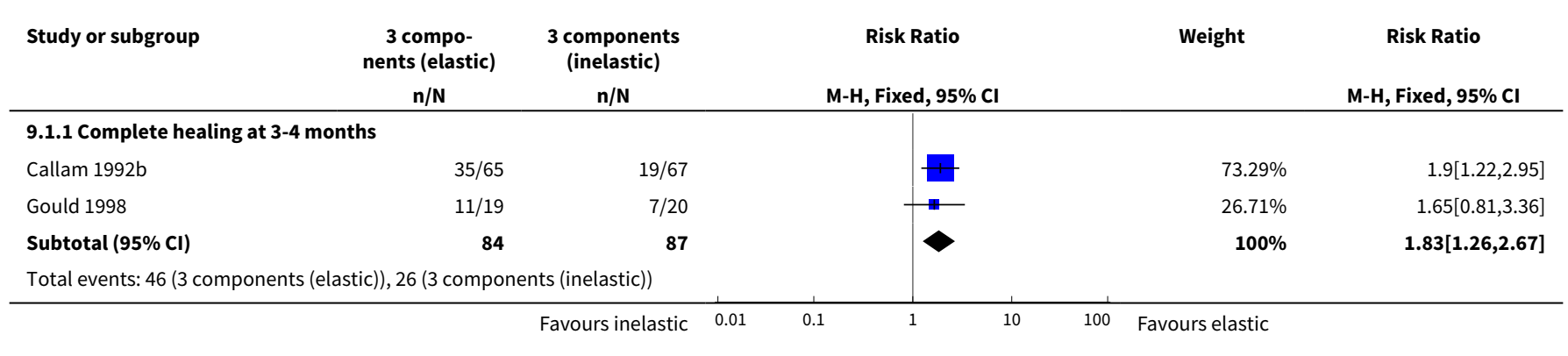




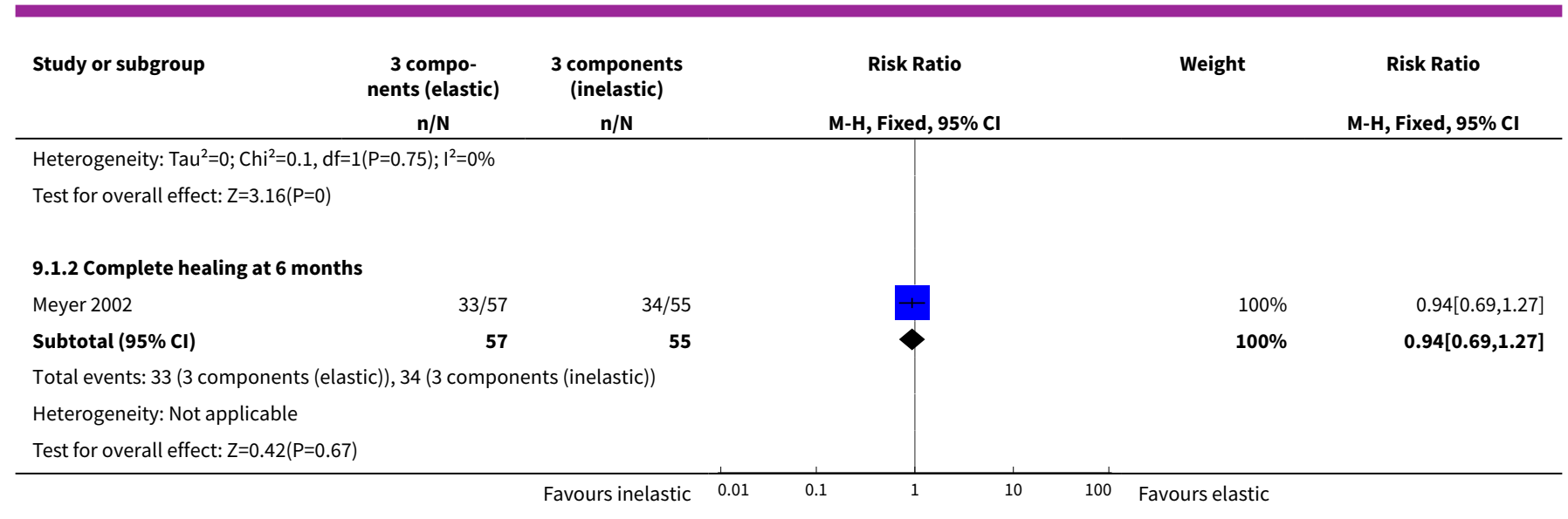

Comparison 10. 3 components including paste bandage vs 3 components including inelastic (short-stretch) bandage

\begin{tabular}{lllll}
\hline Outcome or subgroup title & $\begin{array}{l}\text { No. of } \\
\text { studies }\end{array}$ & $\begin{array}{l}\text { No. of par- } \\
\text { ticipants }\end{array}$ & Statistical method & Effect size \\
\hline 1 Limbs with complete healing at 3 months & 1 & 51 & Risk Ratio (M-H, Fixed, 95\% Cl) & $1.73[0.74,4.06]$ \\
\hline
\end{tabular}

Analysis 10.1. Comparison 103 components including paste bandage vs 3 components including inelastic (short-stretch) bandage, Outcome 1 Limbs with complete healing at 3 months.

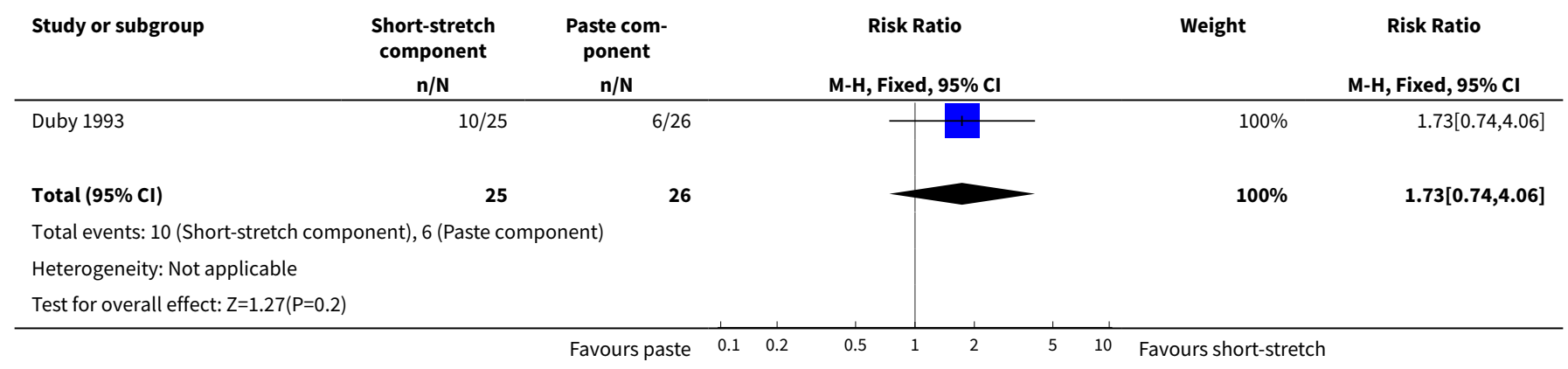

\section{Comparison 11. Charing Cross 4LB vs other 4LB}

\begin{tabular}{lllll}
\hline Outcome or subgroup title & $\begin{array}{l}\text { No. of } \\
\text { studies }\end{array}$ & $\begin{array}{l}\text { No. of } \\
\text { partici- } \\
\text { pants }\end{array}$ & Statistical method & Effect size \\
\hline $\begin{array}{l}1 \text { Patients/limbs with complete healing } \\
\text { during trial }\end{array}$ & 2 & Risk Ratio (M-H, Fixed, 95\% Cl) & Totals not selected \\
\hline 1.1 Complete healing at 3 months & 2 & Risk Ratio (M-H, Fixed, 95\% Cl) & $0.0[0.0,0.0]$ \\
\hline 1.2 Complete healing at 6 months & 1 & Risk Ratio (M-H, Fixed, $95 \% \mathrm{Cl})$ & $0.0[0.0,0.0]$ \\
\hline
\end{tabular}


Analysis 11.1. Comparison 11 Charing Cross 4LB vs other 4LB, Outcome 1 Patients/limbs with complete healing during trial.

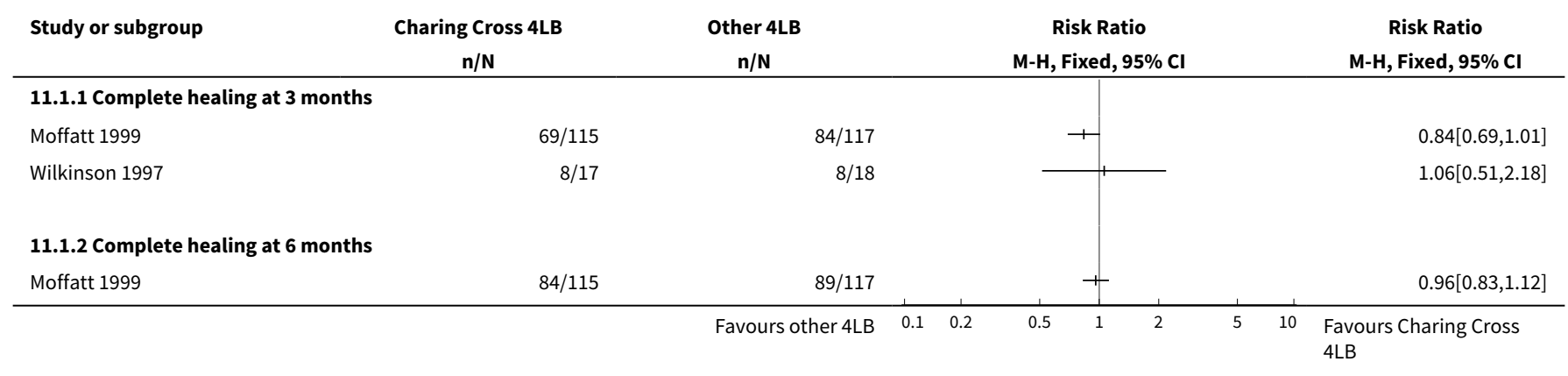

Comparison 12. 4LB vs multi-layer short-stretch bandage (SSB)

\begin{tabular}{|c|c|c|c|c|}
\hline Outcome or subgroup title & $\begin{array}{l}\text { No. of } \\
\text { studies }\end{array}$ & $\begin{array}{l}\text { No. of } \\
\text { partici- } \\
\text { pants }\end{array}$ & Statistical method & Effect size \\
\hline $\begin{array}{l}1 \text { Patients with complete healing during trial period } \\
\text { based on IPD }\end{array}$ & 5 & 797 & Risk Ratio (M-H, Fixed, 95\% Cl) & $0.96[0.88,1.05]$ \\
\hline $\begin{array}{l}2 \text { Limbs completely healed at } 3 \text { months (Duby 1993, } \\
\text { IPD unavailable) }\end{array}$ & 1 & 50 & $\begin{array}{l}\text { Risk Ratio (M-H, Random, 95\% } \\
\mathrm{Cl})\end{array}$ & $1.1[0.57,2.11]$ \\
\hline $\begin{array}{l}3 \text { Hazard ratio estimates for time to healing based } \\
\text { on IPD (fixed-effect) }\end{array}$ & 5 & 797 & Hazard Ratio (Fixed, 95\% Cl) & $1.32[1.09,1.60]$ \\
\hline $\begin{array}{l}4 \text { Hazard ratio estimates for time to healing based } \\
\text { on IPD (random-effects) }\end{array}$ & 5 & 797 & $\begin{array}{l}\text { Hazard Ratio (Random, 95\% } \\
\mathrm{Cl} \text { ) }\end{array}$ & $1.30[0.94,1.80]$ \\
\hline $\begin{array}{l}5 \text { Hazard ratio estimates for time to healing based } \\
\text { on IPD (fixed-effect, Partsch } 2001 \text { removed) }\end{array}$ & 4 & 685 & Hazard Ratio (Fixed, 95\% Cl) & $1.47[1.20,1.81]$ \\
\hline $\begin{array}{l}6 \text { Hazard ratio estimates for time to healing based } \\
\text { on IPD (random-effects, Partsch } 2001 \text { removed) }\end{array}$ & 4 & 685 & $\begin{array}{l}\text { Hazard Ratio (Random, 95\% } \\
\mathrm{Cl} \text { ) }\end{array}$ & $1.47[1.20,1.81]$ \\
\hline $\begin{array}{l}7 \text { Incidence of any type of adverse event based on } \\
\text { IPD (fixed-effect) }\end{array}$ & 2 & 546 & $\begin{array}{l}\text { Odds Ratio (M-H, Fixed, 95\% } \\
\mathrm{Cl})\end{array}$ & $1.15[0.81,1.62]$ \\
\hline $\begin{array}{l}8 \text { Incidence of any type of adverse event based on } \\
\text { IPD (random effects) }\end{array}$ & 2 & 546 & $\begin{array}{l}\text { Odds Ratio (M-H, Random, } \\
95 \% \mathrm{Cl})\end{array}$ & $1.11[0.72,1.72]$ \\
\hline $\begin{array}{l}9 \text { Incidence of bandage-related adverse events } \\
\text { based on IPD }\end{array}$ & 2 & & $\begin{array}{l}\text { Odds Ratio (M-H, Fixed, 95\% } \\
\mathrm{Cl})\end{array}$ & Subtotals only \\
\hline $\begin{array}{l}10 \text { Number of adverse events (any type) based on } \\
\text { IPD (fixed-effect) }\end{array}$ & 2 & 546 & $\begin{array}{l}\text { Mean Difference (IV, Fixed, 95\% } \\
\mathrm{CI} \text { ) }\end{array}$ & $\begin{array}{l}-0.10[-0.32 \\
0.12]\end{array}$ \\
\hline $\begin{array}{l}11 \text { Number of adverse events (any type) based on } \\
\text { IPD (random-effects) }\end{array}$ & 2 & 546 & $\begin{array}{l}\text { Mean Difference (IV, Random, } \\
95 \% \mathrm{CI})\end{array}$ & $\begin{array}{l}-0.21[-0.68 \\
0.27]\end{array}$ \\
\hline
\end{tabular}




\begin{tabular}{lllll}
\hline Outcome or subgroup title & $\begin{array}{l}\text { No. of } \\
\text { studies }\end{array}$ & $\begin{array}{l}\text { No. of } \\
\text { partici- } \\
\text { pants }\end{array}$ & Statistical method & Effect size \\
\hline $\begin{array}{l}12 \text { Number of adverse events (bandage-related) } \\
\text { based on IPD }\end{array}$ & 2 & & $\begin{array}{l}\text { Mean Difference (IV, Random, } \\
95 \% \mathrm{Cl})\end{array}$ & Subtotals only \\
\hline
\end{tabular}

Analysis 12.1. Comparison 12 4LB vs multi-layer short-stretch bandage (SSB), Outcome 1 Patients with complete healing during trial period based on IPD.

\begin{tabular}{|c|c|c|c|c|c|}
\hline Study or subgroup & $\begin{array}{l}\text { SSB } \\
n / N\end{array}$ & $\begin{array}{l}\text { 4LB } \\
\mathrm{n} / \mathrm{N}\end{array}$ & $\begin{array}{c}\text { Risk Ratio } \\
\text { M-H, Fixed, 95\% Cl }\end{array}$ & Weight & $\begin{array}{c}\text { Risk Ratio } \\
\text { M-H, Fixed, 95\% Cl }\end{array}$ \\
\hline Franks 2004 & $63 / 84$ & $60 / 75$ & $\rightarrow$ & $22.42 \%$ & $0.94[0.79,1.11]$ \\
\hline Iglesias 2004 & $147 / 192$ & $157 / 195$ & & $55.1 \%$ & $0.95[0.86,1.06]$ \\
\hline Partsch 2001 & $43 / 59$ & $33 / 53$ & 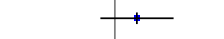 & $12.3 \%$ & $1.17[0.9,1.52]$ \\
\hline Scriven 1998 & $12 / 23$ & $17 / 27$ & \begin{tabular}{l|l} 
\\
\end{tabular} & $5.53 \%$ & $0.83[0.51,1.35]$ \\
\hline Ukat 2003 & $10 / 45$ & $13 / 44$ & ए & $4.65 \%$ & $0.75[0.37,1.53]$ \\
\hline Total $(95 \% \mathrm{CI})$ & 403 & 394 & & $100 \%$ & $0.96[0.88,1.05]$ \\
\hline \multicolumn{6}{|c|}{ Total events: 275 (SSB), 280 (4LB) } \\
\hline \multicolumn{6}{|c|}{ Heterogeneity: $\mathrm{Tau}^{2}=0 ; \mathrm{Chi}^{2}=3.13, \mathrm{df}=4(\mathrm{P}=0.54) ; \mathrm{I}^{2}=0 \%$} \\
\hline \multicolumn{6}{|c|}{ Test for overall effect: $Z=0.96(P=0.34)$} \\
\hline
\end{tabular}

Analysis 12.2. Comparison 12 4LB vs multi-layer short-stretch bandage (SSB), Outcome 2 Limbs completely healed at 3 months (Duby 1993, IPD unavailable).

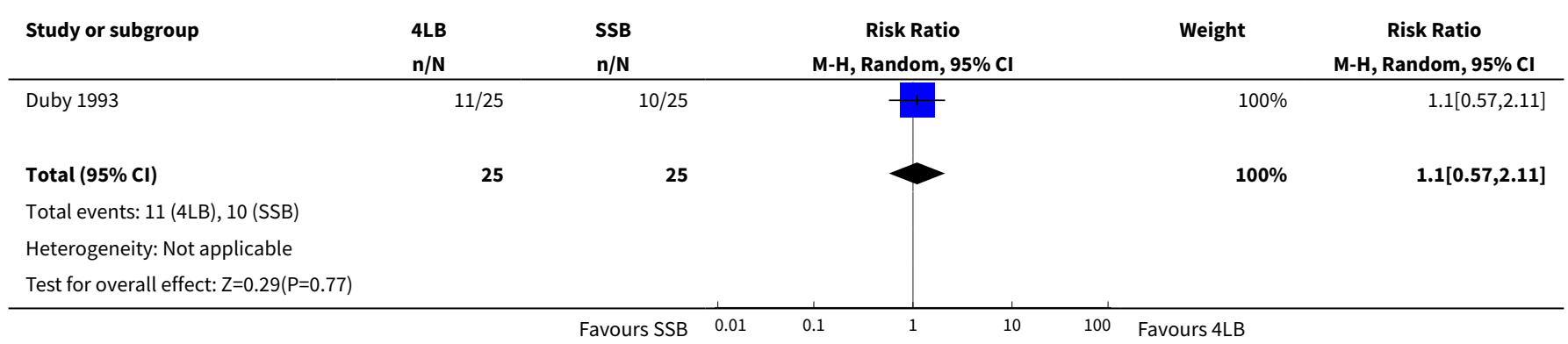

Analysis 12.3. Comparison 12 4LB vs multi-layer short-stretch bandage (SSB), Outcome 3 Hazard ratio estimates for time to healing based on IPD (fixed-effect).

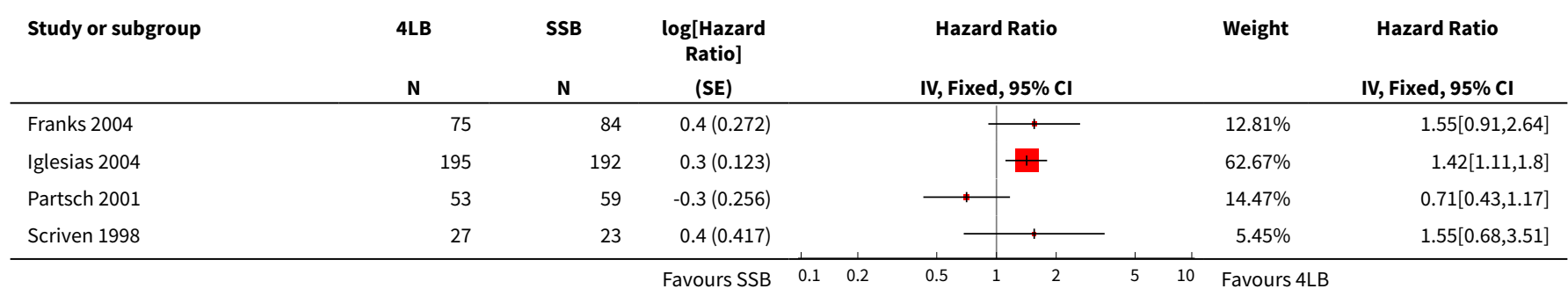




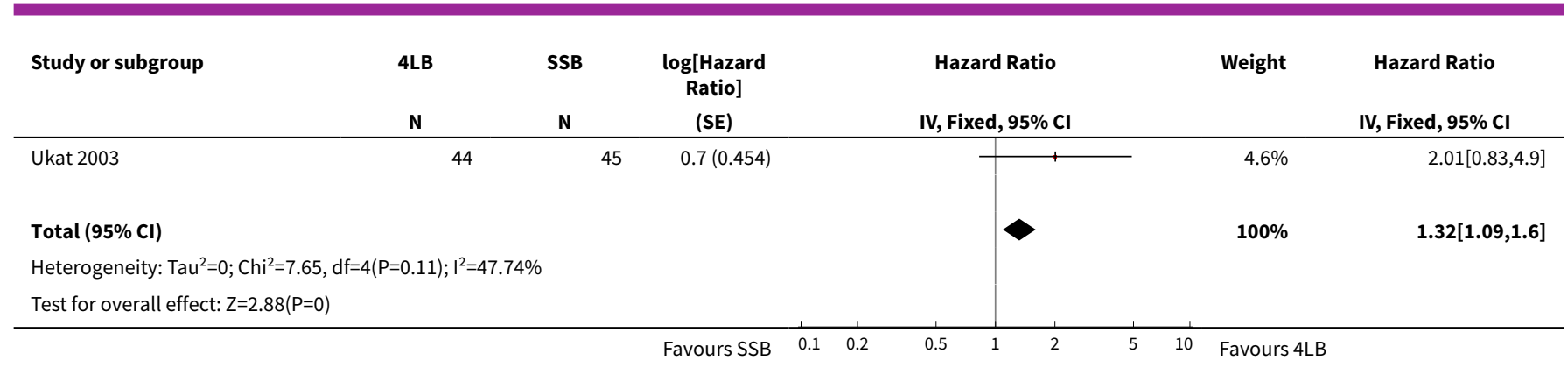

Analysis 12.4. Comparison 12 LLB vs multi-layer short-stretch bandage (SSB), Outcome 4 Hazard ratio estimates for time to healing based on IPD (random-effects).

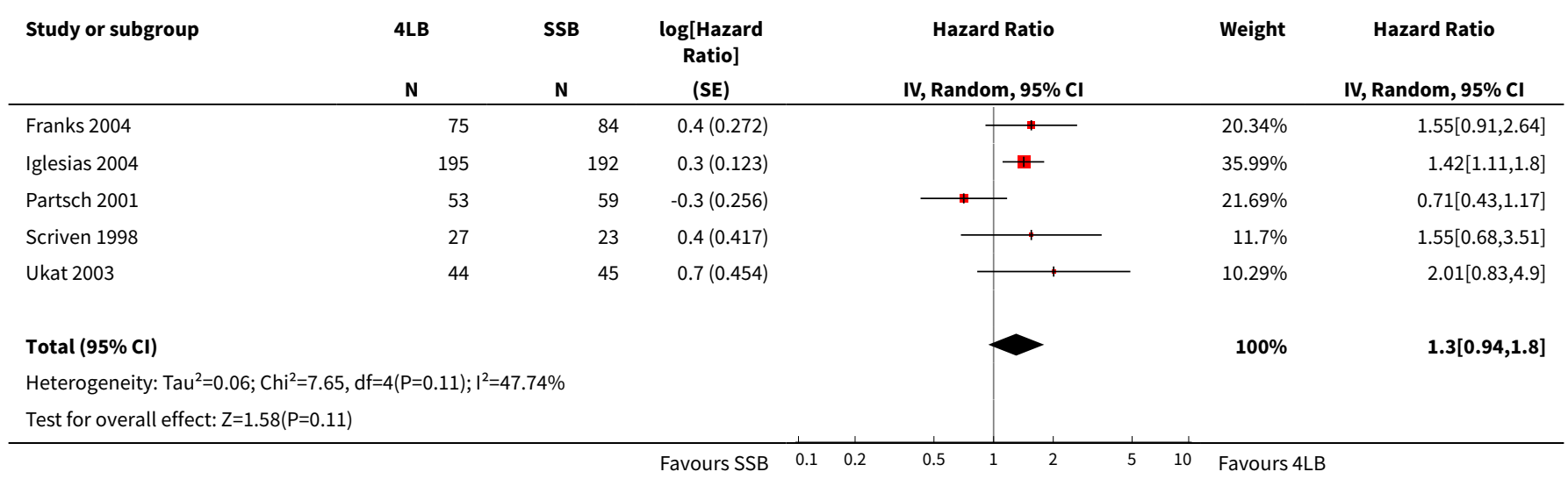

Analysis 12.5. Comparison 12 4LB vs multi-layer short-stretch bandage (SSB), Outcome 5 Hazard ratio estimates for time to healing based on IPD (fixed-effect, Partsch 2001 removed).

\begin{tabular}{|c|c|c|c|c|c|c|}
\hline \multirow[t]{2}{*}{ Study or subgroup } & \multirow{2}{*}{$\begin{array}{l}\text { 4LB } \\
\mathrm{N} \\
\end{array}$} & \multirow{2}{*}{$\begin{array}{l}\text { SSB } \\
\text { N } \\
\end{array}$} & \multirow{2}{*}{$\begin{array}{c}\log [\text { Hazard } \\
\text { Ratio] } \\
\text { (SE) }\end{array}$} & Hazard Ratio & \multirow[t]{2}{*}{ Weight } & \multirow{2}{*}{$\begin{array}{c}\text { Hazard Ratio } \\
\text { IV, Fixed, } 95 \% \mathrm{CI}\end{array}$} \\
\hline & & & & IV, Fixed, $95 \% \mathrm{CI}$ & & \\
\hline Franks 2004 & 75 & 84 & $0.4(0.272)$ & $\longrightarrow$ & $14.98 \%$ & $1.55[0.91,2.64]$ \\
\hline Iglesias 2004 & 195 & 192 & $0.3(0.123)$ & $\rightarrow$ & $73.27 \%$ & $1.42[1.11,1.8]$ \\
\hline Scriven 1998 & 27 & 23 & $0.4(0.417)$ & & $6.37 \%$ & $1.55[0.68,3.51]$ \\
\hline Ukat 2003 & 44 & 45 & $0.7(0.454)$ & & $5.38 \%$ & $2.01[0.83,4.9]$ \\
\hline Total $(95 \% \mathrm{Cl})$ & & & & & $100 \%$ & $1.47[1.2,1.81]$ \\
\hline \multicolumn{7}{|c|}{ Heterogeneity: $\mathrm{Tau}^{2}=0 ; \mathrm{Chi}^{2}=0.62, \mathrm{df}=3(\mathrm{P}=0.89) ; \mathrm{I}^{2}=0 \%$} \\
\hline Test for overall effect & & & & & & \\
\hline
\end{tabular}


Analysis 12.6. Comparison 12 4LB vs multi-layer short-stretch bandage (SSB), Outcome 6 Hazard ratio estimates for time to healing based on IPD (random-effects, Partsch 2001 removed).

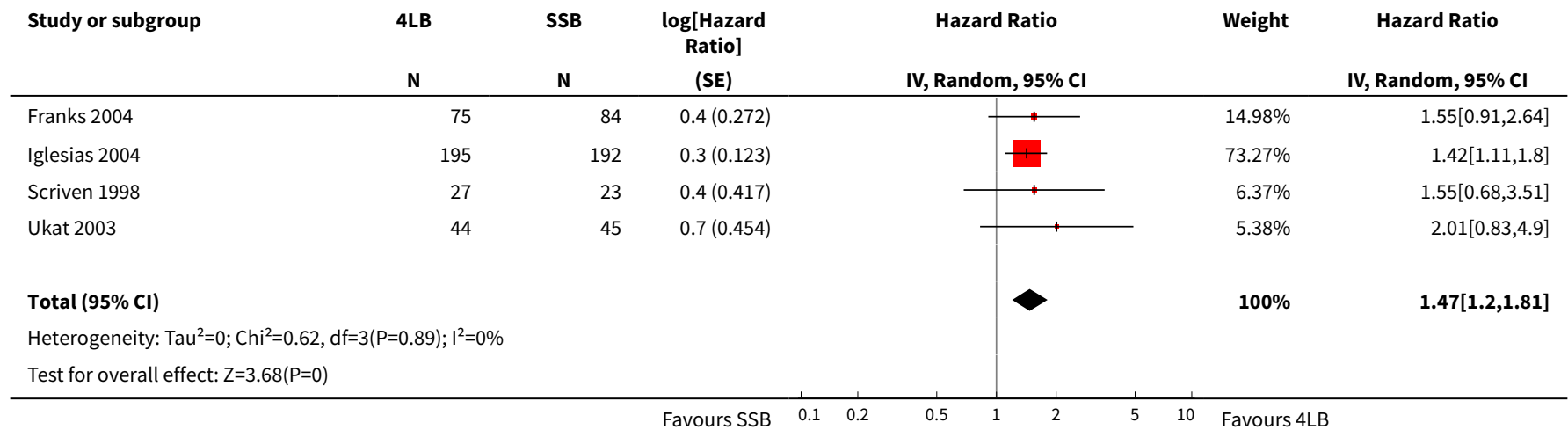

Analysis 12.7. Comparison 12 4LB vs multi-layer short-stretch bandage (SSB), Outcome 7 Incidence of any type of adverse event based on IPD (fixed-effect).

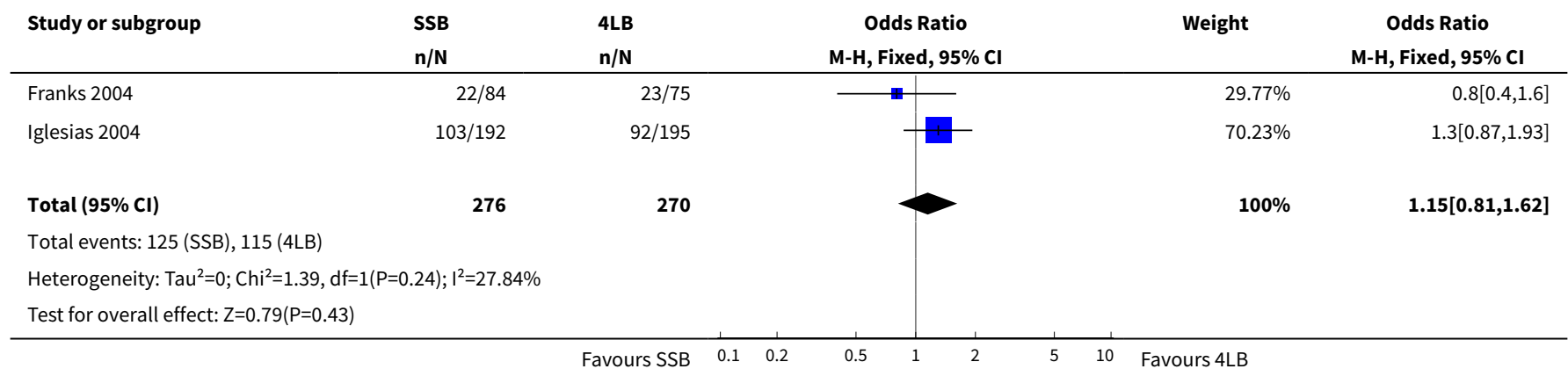

Analysis 12.8. Comparison 12 LLB vs multi-layer short-stretch bandage (SSB), Outcome 8 Incidence of any type of adverse event based on IPD (random effects).

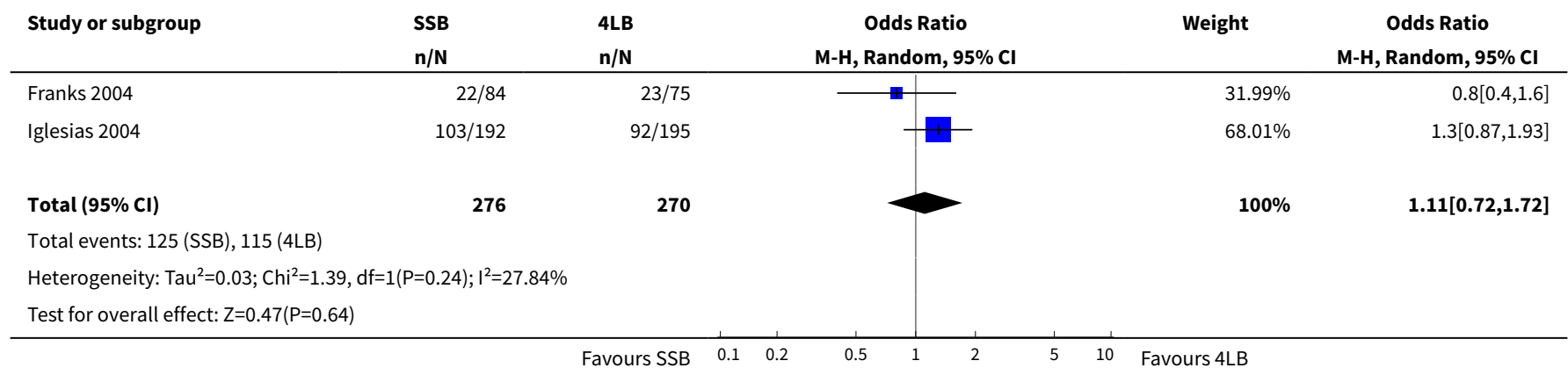


Analysis 12.9. Comparison 124 LB vs multi-layer short-stretch bandage (SSB), Outcome 9 Incidence of bandage-related adverse events based on IPD.

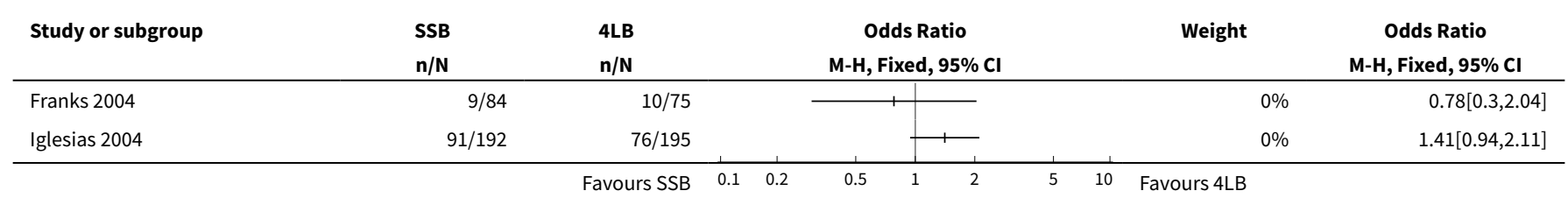

Analysis 12.10. Comparison 12 4LB vs multi-layer short-stretch bandage (SSB), Outcome 10 Number of adverse events (any type) based on IPD (fixed-effect).

\begin{tabular}{|c|c|c|c|c|c|c|c|}
\hline \multirow[t]{2}{*}{ Study or subgroup } & \multicolumn{2}{|c|}{ 4LB } & \multicolumn{2}{|c|}{ SSB } & \multirow{2}{*}{$\begin{array}{c}\text { Mean Difference } \\
\text { Fixed, } 95 \% \mathrm{Cl}\end{array}$} & \multirow[t]{2}{*}{ Weight } & \multirow{2}{*}{$\begin{array}{c}\text { Mean Difference } \\
\text { Fixed, } 95 \% \mathrm{Cl}\end{array}$} \\
\hline & $\mathbf{N}$ & $\operatorname{Mean}(S D)$ & $\mathbf{N}$ & Mean(SD) & & & \\
\hline Franks 2004 & 75 & $0.4(0.7)$ & 84 & $0.4(0.9)$ & & $86.26 \%$ & $-0.03[-0.27,0.21]$ \\
\hline Iglesias 2004 & 195 & $1.6(2.6)$ & 192 & $2.1(3.4)$ & $\longrightarrow$ & $13.74 \%$ & $-0.54[-1.14,0.06]$ \\
\hline 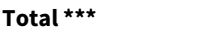 & 270 & & 276 & & & $100 \%$ & $-0.1[-0.32,0.12]$ \\
\hline \multicolumn{8}{|c|}{ Heterogeneity: $\mathrm{Tau}^{2}=0 ; \mathrm{Chi}^{2}=2.36, \mathrm{df}=1(\mathrm{P}=0.12) ; \mathrm{I}^{2}=57.55 \%$} \\
\hline \multicolumn{3}{|c|}{ Test for overall effect: $Z=0.87(P=0.38)$} & & & & & \\
\hline
\end{tabular}

Analysis 12.11. Comparison 12 4LB vs multi-layer short-stretch bandage (SSB), Outcome 11 Number of adverse events (any type) based on IPD (random-effects).

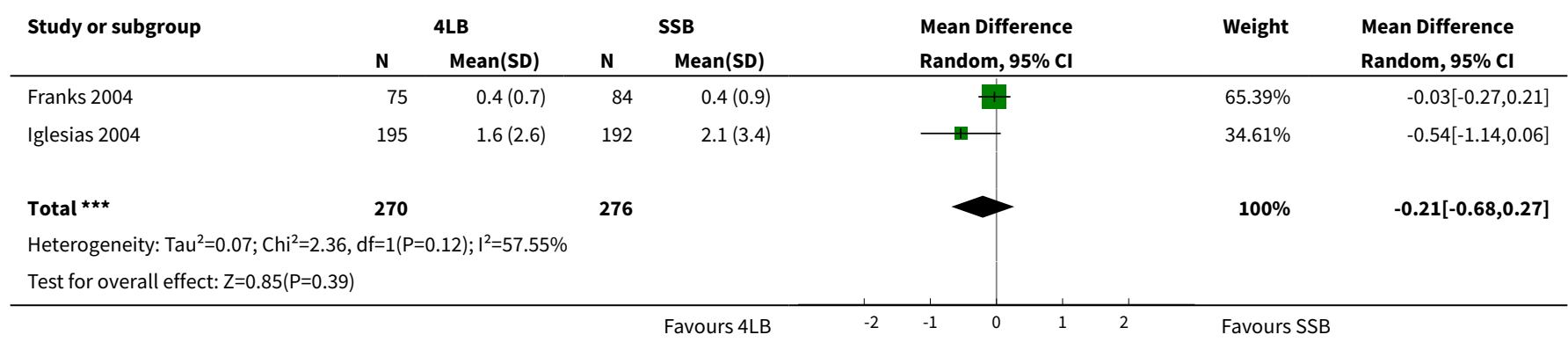

Analysis 12.12. Comparison 12 4LB vs multi-layer short-stretch bandage (SSB), Outcome 12 Number of adverse events (bandage-related) based on IPD.

\begin{tabular}{|c|c|c|c|c|c|c|c|}
\hline \multirow[t]{2}{*}{ Study or subgroup } & \multicolumn{2}{|c|}{ 4LB } & \multicolumn{2}{|c|}{ SSB } & \multirow{2}{*}{$\begin{array}{l}\text { Mean Difference } \\
\text { Random, } 95 \% \mathrm{CI}\end{array}$} & \multirow[t]{2}{*}{ Weight } & \multirow{2}{*}{$\begin{array}{l}\text { Mean Difference } \\
\text { Random, } 95 \% \mathrm{Cl}\end{array}$} \\
\hline & $\mathbf{N}$ & Mean(SD) & $\mathbf{N}$ & Mean(SD) & & & \\
\hline Franks 2004 & 75 & $0.2(0.5)$ & 84 & $0.1(0.4)$ & + & $0 \%$ & $0.04[-0.09,0.17]$ \\
\hline Iglesias 2004 & 195 & $1.3(2.4)$ & 192 & $1.8(3.1)$ & $\longrightarrow$ & $0 \%$ & $-0.45[-1.01,0.11]$ \\
\hline
\end{tabular}


Comparison 13. Four-layer bandage (4LB) vs compression system with paste bandage as the base

\begin{tabular}{|c|c|c|c|c|}
\hline Outcome or subgroup title & $\begin{array}{l}\text { No. of } \\
\text { studies }\end{array}$ & $\begin{array}{l}\text { No. of } \\
\text { partici- } \\
\text { pants }\end{array}$ & Statistical method & Effect size \\
\hline $\begin{array}{l}1 \text { Patients/limbs with complete healing } \\
\text { during trial }\end{array}$ & 4 & & Risk Ratio (M-H, Fixed, 95\% Cl) & Subtotals only \\
\hline $\begin{array}{l}\text { 1.1 Patients/limbs with complete healing } \\
\text { at } 3 \text { months }\end{array}$ & 2 & 71 & Risk Ratio (M-H, Fixed, 95\% Cl) & $1.34[0.78,2.28]$ \\
\hline $\begin{array}{l}1.2 \text { Patients with complete healing at } 6 \\
\text { months }\end{array}$ & 1 & 68 & Risk Ratio (M-H, Fixed, 95\% Cl) & $1.13[0.82,1.57]$ \\
\hline $\begin{array}{l}\text { 1.3 Patients with complete healing at } 1 \\
\text { year }\end{array}$ & 1 & 133 & Risk Ratio (M-H, Fixed, 95\% Cl) & $0.82[0.66,1.01]$ \\
\hline $\begin{array}{l}2 \text { Patients/limbs with complete healing at } \\
3 \text { months (random-effects) }\end{array}$ & 2 & 71 & Risk Ratio (M-H, Random, 95\% Cl) & $1.23[0.54,2.82]$ \\
\hline $\begin{array}{l}3 \text { Percentage reduction of baseline ulcer } \\
\text { area at } 6 \text { months }\end{array}$ & 1 & 68 & Mean Difference (IV, Fixed, 95\% Cl) & $\begin{array}{l}54.50[-9.17 \\
118.17]\end{array}$ \\
\hline 4 Healing rate (pooled) & 2 & 78 & Std. Mean Difference (IV, Fixed, 95\% CI) & $0.52[0.06,0.97]$ \\
\hline
\end{tabular}

Analysis 13.1. Comparison 13 Four-layer bandage (4LB) vs compression system with paste bandage as the base, Outcome 1 Patients/limbs with complete healing during trial.

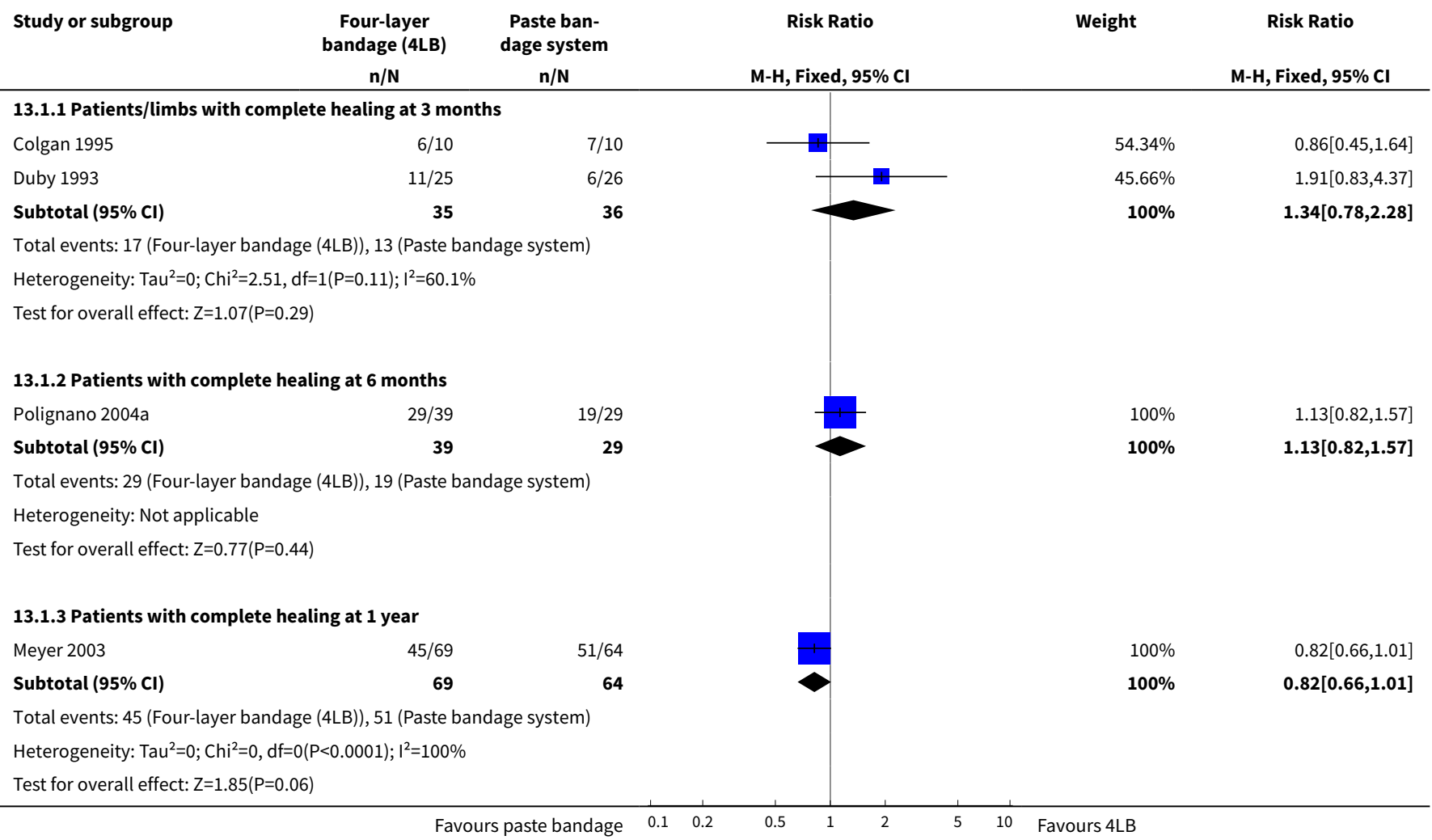


Analysis 13.2. Comparison 13 Four-layer bandage (4LB) vs compression system with paste bandage as the base, Outcome 2 Patients/limbs with complete healing at 3 months (random-effects).

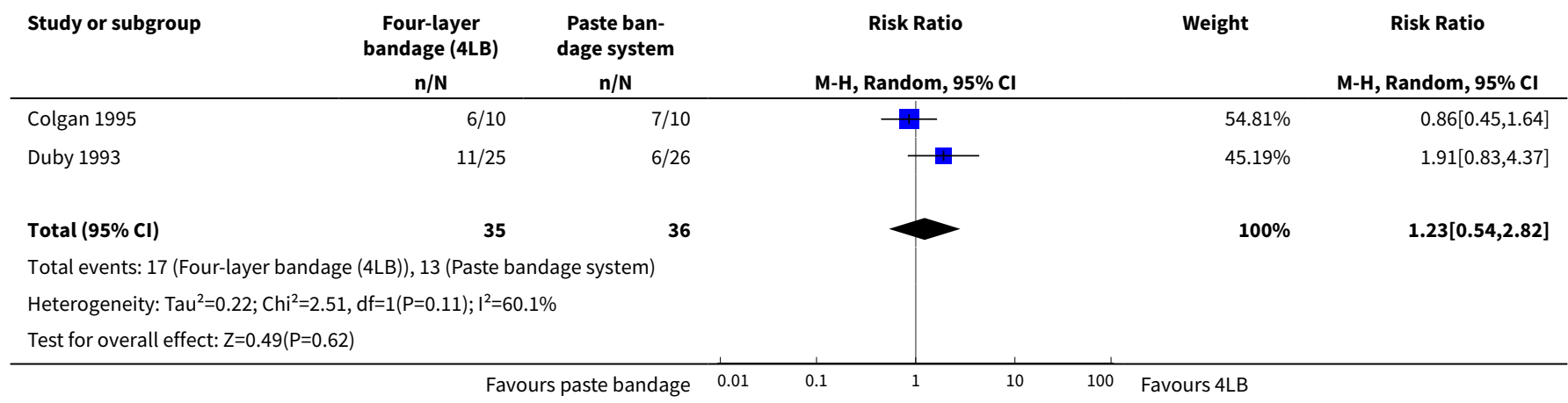

Analysis 13.3. Comparison 13 Four-layer bandage (4LB) vs compression system with paste bandage as the base, Outcome 3 Percentage reduction of baseline ulcer area at 6 months.

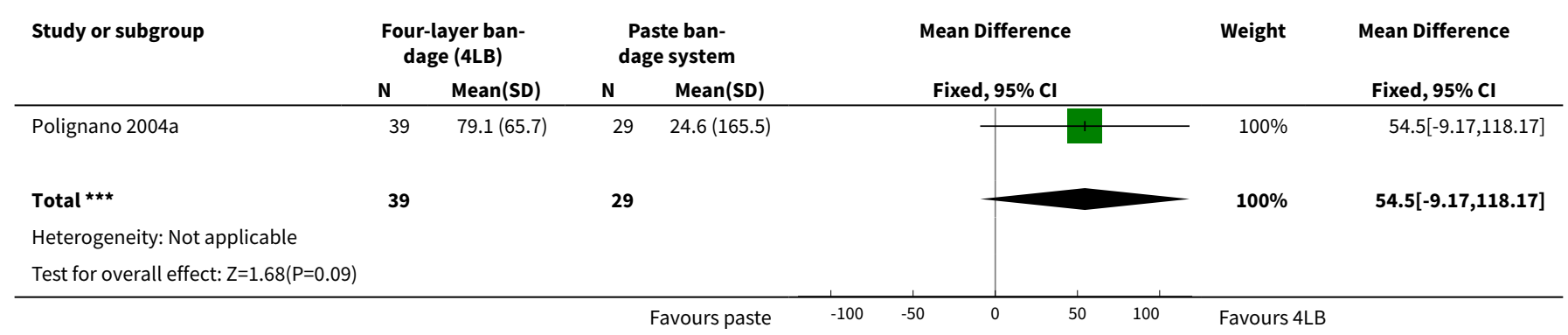

Analysis 13.4. Comparison 13 Four-layer bandage (4LB) vs compression system with paste bandage as the base, Outcome 4 Healing rate (pooled).

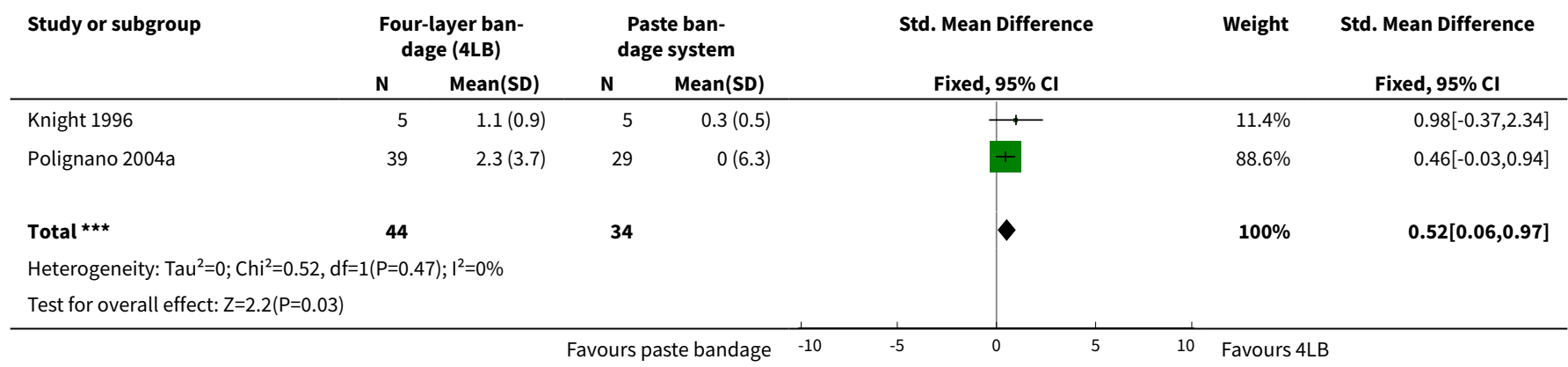


Comparison 14. Adjustable inelastic compression boot vs other compression system

\begin{tabular}{lllll}
\hline Outcome or subgroup title & $\begin{array}{l}\text { No. of } \\
\text { studies }\end{array}$ & $\begin{array}{l}\text { No. of } \\
\text { partici- } \\
\text { pants }\end{array}$ & Statistical method & Effect size \\
\hline 1 Healing rate & 2 & Mean Difference (IV, Fixed, $95 \% \mathrm{Cl})$ & Totals not selected \\
\hline $1.1 \mathrm{~cm}^{2}$ per week & 1 & Mean Difference (IV, Fixed, $95 \% \mathrm{Cl})$ & $0.0[0.0,0.0]$ \\
\hline $1.2 \mathrm{~cm}^{2}$ per day & 1 & Mean Difference (IV, Fixed, $95 \% \mathrm{Cl})$ & $0.0[0.0,0.0]$ \\
\hline 1.3 percentage per day & 1 & Mean Difference (IV, Fixed, $95 \% \mathrm{Cl})$ & $0.0[0.0,0.0]$ \\
\hline $1.4 \mathrm{~cm}$ per day (linear rate) & 1 & Mean Difference (IV, Fixed, $95 \% \mathrm{Cl})$ & $0.0[0.0,0.0]$
\end{tabular}

Analysis 14.1. Comparison 14 Adjustable inelastic compression boot vs other compression system, Outcome 1 Healing rate.

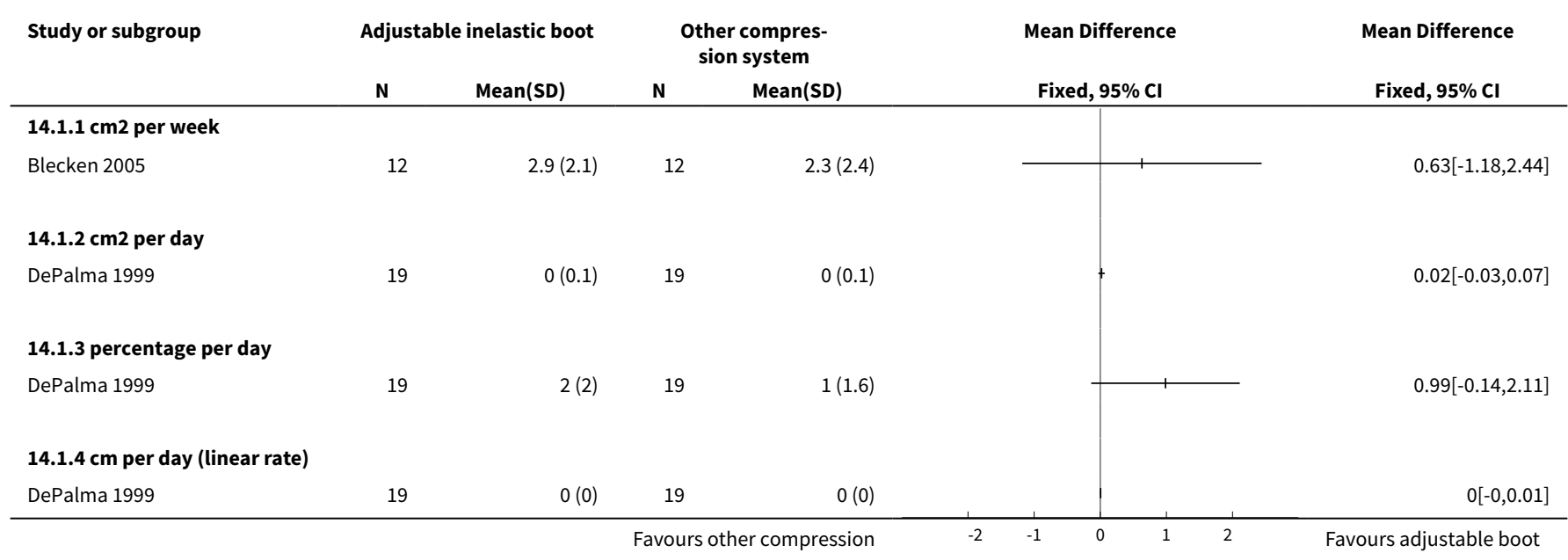

\section{Comparison 15. Single-layer compression stocking vs paste bandage system}

\begin{tabular}{lllll}
\hline Outcome or subgroup title & $\begin{array}{l}\text { No. of } \\
\text { studies }\end{array}$ & $\begin{array}{l}\text { No. of } \\
\text { partici- } \\
\text { pants }\end{array}$ & Statistical method & Effect size \\
\hline $\begin{array}{l}1 \text { Complete healing in trial period } \\
\text { (varying lengths) }\end{array}$ & 2 & & Risk Ratio (M-H, Fixed, 95\% Cl) & Totals not selected \\
\hline $\begin{array}{l}1.1 \text { Patients with complete healing at } 4 \\
\text { months }\end{array}$ & 1 & Risk Ratio (M-H, Fixed, 95\% Cl) & $0.0[0.0,0.0]$ \\
\hline $\begin{array}{l}1.2 \text { Patients with complete healing at } \\
18 \text { months }\end{array}$ & 1 & Risk Ratio (M-H, Fixed, 95\% Cl) & $0.0[0.0,0.0]$ \\
\hline \begin{tabular}{l}
2 Healing rate $\left(\mathrm{cm}^{2}\right.$ per week) \\
\hline
\end{tabular} & 1 & 60 & Mean Difference (IV, Fixed, $95 \% \mathrm{Cl})$ & $-0.12[-0.41,0.17]$ \\
\hline
\end{tabular}


Analysis 15.1. Comparison 15 Single-layer compression stocking vs paste bandage system, Outcome 1 Complete healing in trial period (varying lengths).

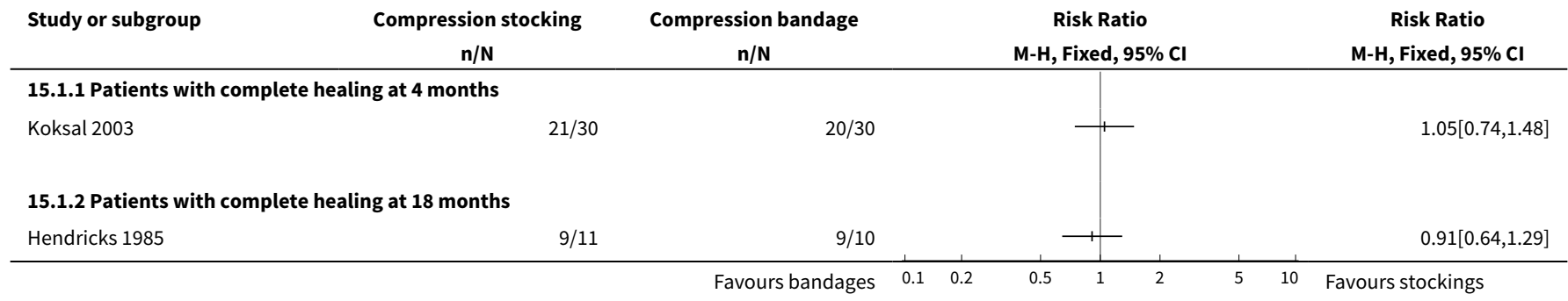

\section{Analysis 15.2. Comparison 15 Single-layer compression stocking} vs paste bandage system, Outcome 2 Healing rate ( $\mathrm{cm}^{2}$ per week).

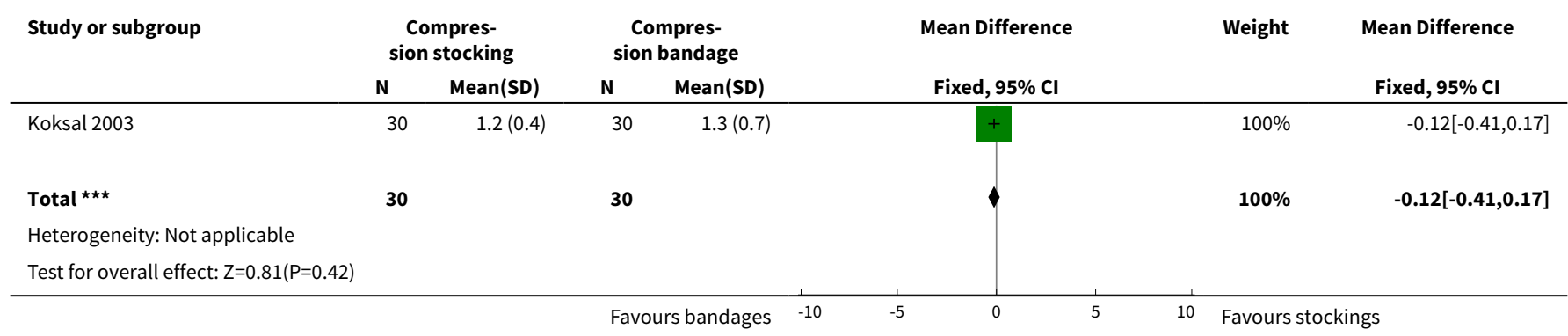

Comparison 16. Low-compression stocking vs short-stretch bandage (SSB)

\begin{tabular}{lllll}
\hline Outcome or subgroup title & $\begin{array}{l}\text { No. of } \\
\text { studies }\end{array}$ & $\begin{array}{l}\text { No. of } \\
\text { partici- } \\
\text { pants }\end{array}$ & Statistical method & Effect size \\
\hline $\begin{array}{l}1 \text { Patients with complete healing at } 3 \\
\text { months }\end{array}$ & 1 & Risk Ratio (M-H, Fixed, 95\% Cl) & Totals not selected \\
\hline $\begin{array}{l}2 \text { Patients with complete healing at } 6 \\
\text { months }\end{array}$ & 1 & Risk Ratio (M-H, Fixed, 95\% Cl) & Totals not selected \\
\hline
\end{tabular}

Analysis 16.1. Comparison 16 Low-compression stocking vs short-stretch bandage (SSB), Outcome 1 Patients with complete healing at 3 months.

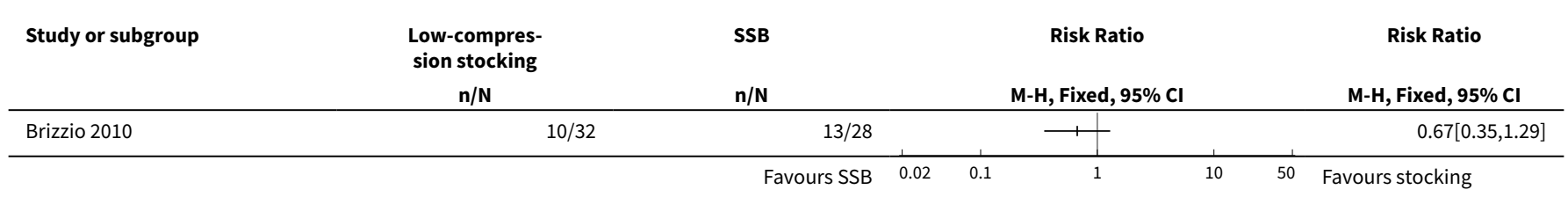


Analysis 16.2. Comparison 16 Low-compression stocking vs short-stretch bandage (SSB), Outcome 2 Patients with complete healing at 6 months.

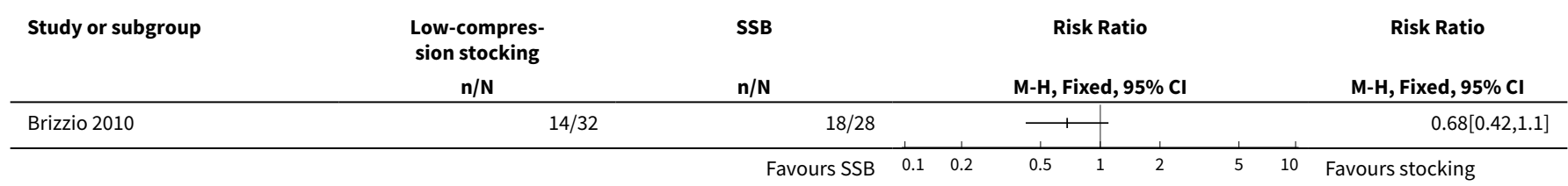

Comparison 17. High-compression stockings vs short-stretch bandage (SSB)

\begin{tabular}{llllll}
\hline Outcome or subgroup title & $\begin{array}{l}\text { No. of } \\
\text { studies }\end{array}$ & $\begin{array}{l}\text { No. of } \\
\text { partici- } \\
\text { pants }\end{array}$ & Statistical method & Effect size \\
\hline $\begin{array}{l}1 \text { Patients with complete healing at 2-4 months } \\
\text { (fixed-effect) }\end{array}$ & 4 & 317 & Risk Ratio (M-H, Fixed, 95\% Cl) & $1.62[1.26,2.10]$ \\
\hline $\begin{array}{l}2 \text { Patients with complete healing at 2-4 months } \\
\text { (random-effects) }\end{array}$ & 4 & 317 & Risk Ratio (M-H, Random, 95\% Cl) & $1.66[1.07,2.58]$ \\
\hline $\begin{array}{l}3 \text { Percentage reduction of baseline ulcer area at } \\
3 \text { months }\end{array}$ & 1 & 119 & $\begin{array}{l}\text { Mean Difference (IV, Fixed, 95\% } \\
\text { Cl) }\end{array}$ & $\begin{array}{l}23.4[-1.32, \\
48.12]\end{array}$ \\
\hline
\end{tabular}

Analysis 17.1. Comparison 17 High-compression stockings vs short-stretch bandage (SSB), Outcome 1 Patients with complete healing at 2-4 months (fixed-effect).

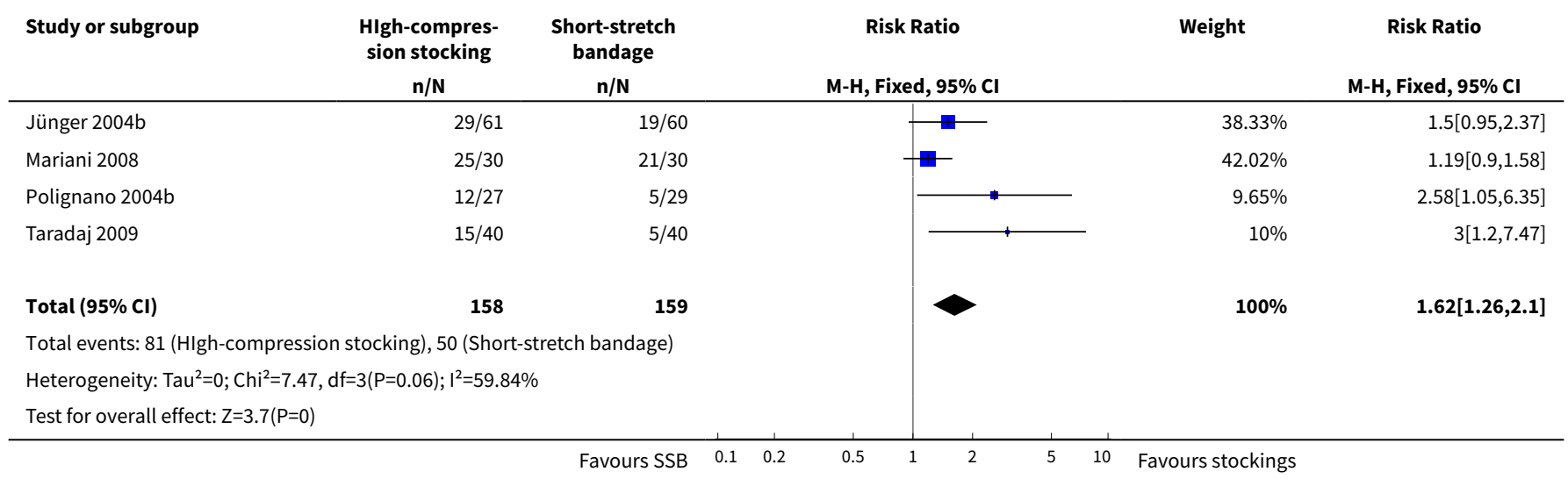


Analysis 17.2. Comparison 17 High-compression stockings vs short-stretch bandage (SSB), Outcome 2 Patients with complete healing at 2-4 months (random-effects).

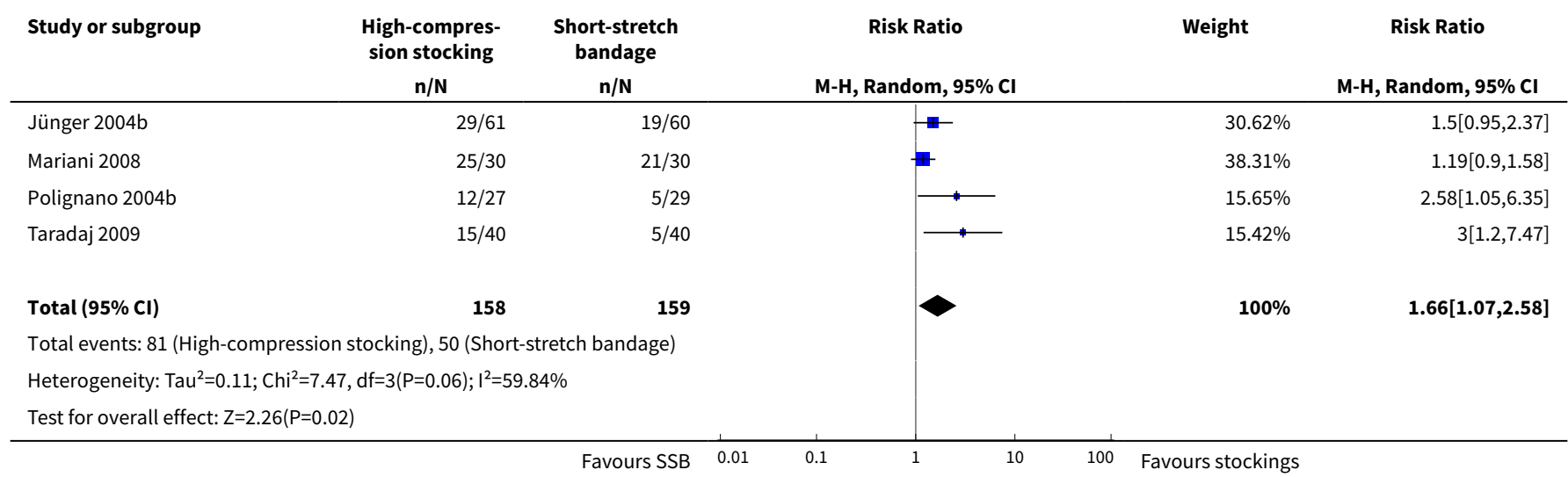

Analysis 17.3. Comparison 17 High-compression stockings vs short-stretch bandage (SSB), Outcome 3 Percentage reduction of baseline ulcer area at 3 months.

\begin{tabular}{|c|c|c|c|c|c|c|c|c|}
\hline \multirow[t]{2}{*}{ Study or subgroup } & \multicolumn{2}{|c|}{ Two-layer stocking } & \multicolumn{2}{|c|}{$\begin{array}{c}\text { Short-stretch } \\
\text { bandage }\end{array}$} & & \multirow{2}{*}{$\begin{array}{c}\text { Mean Difference } \\
\text { Fixed, } 95 \% \mathrm{Cl} \\
\end{array}$} & \multirow[t]{2}{*}{ Weight } & \multirow{2}{*}{$\begin{array}{l}\text { Mean Difference } \\
\text { Fixed, } 95 \% \mathrm{Cl}\end{array}$} \\
\hline & $\mathbf{N}$ & $\operatorname{Mean}(S D)$ & $\mathbf{N}$ & Mean(SD) & & & & \\
\hline Jünger 2004b & 61 & $74.8(42.4)$ & 58 & $51.4(86.7)$ & & & $100 \%$ & $23.4[-1.32,48.12]$ \\
\hline Total $\star \star \star ~$ & 61 & & 58 & & & & $100 \%$ & $23.4[-1.32,48.12]$ \\
\hline \multicolumn{9}{|c|}{ Heterogeneity: Not applicable } \\
\hline \multicolumn{9}{|c|}{ Test for overall effect: $Z=1.86(P=0.06)$} \\
\hline & & & & Favours SSB & -100 & -50 & Favours & \\
\hline
\end{tabular}

\section{Comparison 18. Compression stocking vs two-component bandage system}

\begin{tabular}{lllll}
\hline Outcome or subgroup title & $\begin{array}{l}\text { No. of } \\
\text { studies }\end{array}$ & $\begin{array}{l}\text { No. of par- } \\
\text { ticipants }\end{array}$ & Statistical method & Effect size \\
\hline 1 Patients with complete healing at 3 months & 1 & Risk Ratio (M-H, Fixed, 95\% Cl) & Subtotals only \\
\hline
\end{tabular}

Analysis 18.1. Comparison 18 Compression stocking vs two-component bandage system, Outcome 1 Patients with complete healing at 3 months.

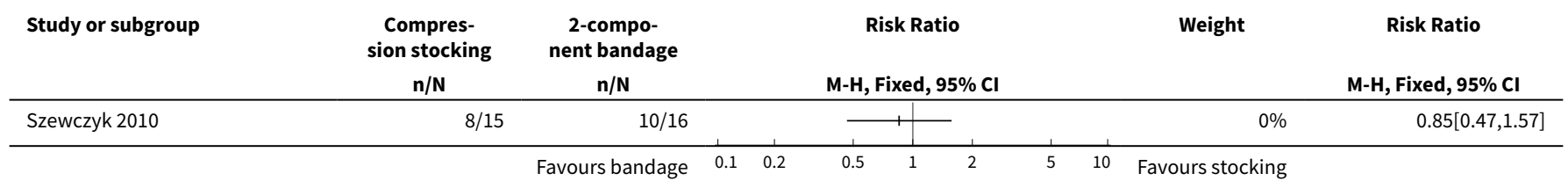


Comparison 19. Compression stocking vs 4LB

\begin{tabular}{llll}
\hline Outcome or subgroup title & $\begin{array}{l}\text { No. of } \\
\text { studies }\end{array}$ & $\begin{array}{l}\text { No. of par- } \\
\text { ticipants }\end{array}$ & Statistical method \\
\hline 1 Patients with complete healing at 3 months & 1 & Risk Ratio (M-H, Fixed, 95\% Cl) & Subtotals only \\
\hline
\end{tabular}

Analysis 19.1. Comparison 19 Compression stocking vs 4LB, Outcome 1 Patients with complete healing at 3 months.

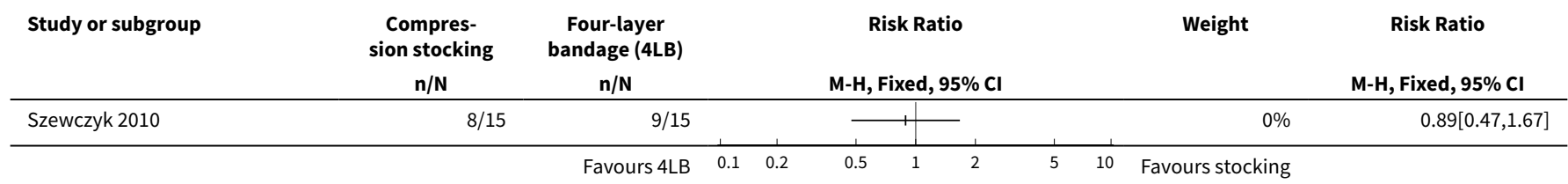

Comparison 20. Tubular compression vs short-stretch bandage (SSB)

\begin{tabular}{lllll}
\hline Outcome or subgroup title & $\begin{array}{l}\text { No. of } \\
\text { studies }\end{array}$ & $\begin{array}{l}\text { No. of par- } \\
\text { ticipants }\end{array}$ & Statistical method & Effect size \\
\hline 1 Patients with complete healing at 3 months & 1 & 178 & Risk Ratio (M-H, Fixed, 95\% Cl) & $0.98[0.76,1.26]$ \\
\hline
\end{tabular}

Analysis 20.1. Comparison 20 Tubular compression vs short-stretch bandage (SSB), Outcome 1 Patients with complete healing at 3 months.

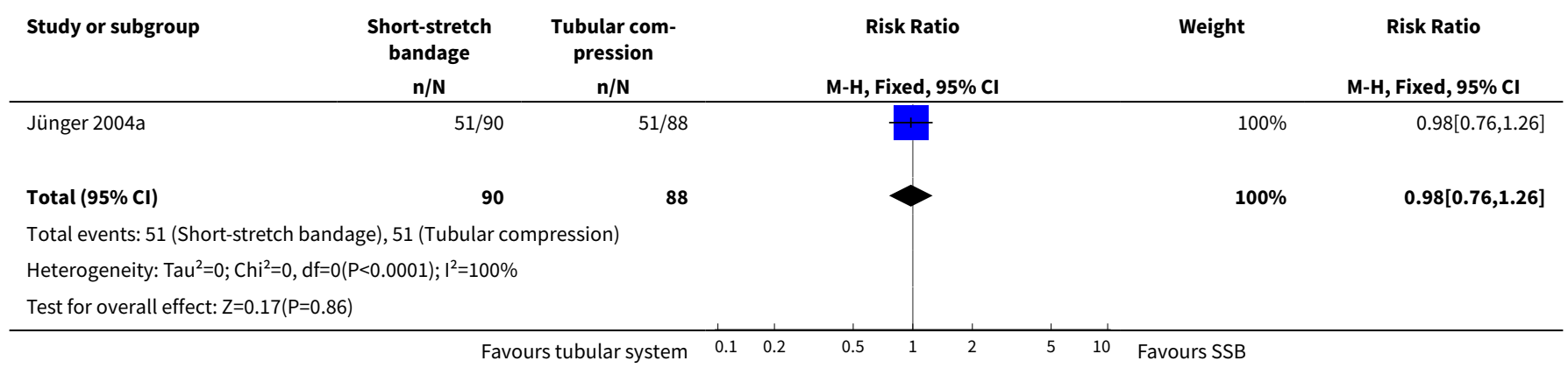

Comparison 21. Tubular compression vs tubular plus 1 elastic bandage

\begin{tabular}{llll}
\hline Outcome or subgroup title & $\begin{array}{l}\text { No. of } \\
\text { studies }\end{array}$ & $\begin{array}{l}\text { No. of par- } \\
\text { ticipants }\end{array}$ & Statistical method \\
\hline 1 Patients with complete healing at six months & 1 & 88 & Risk Ratio (M-H, Fixed, 95\% Cl) \\
\hline
\end{tabular}


Analysis 21.1. Comparison 21 Tubular compression vs tubular plus 1 elastic bandage, Outcome 1 Patients with complete healing at six months.

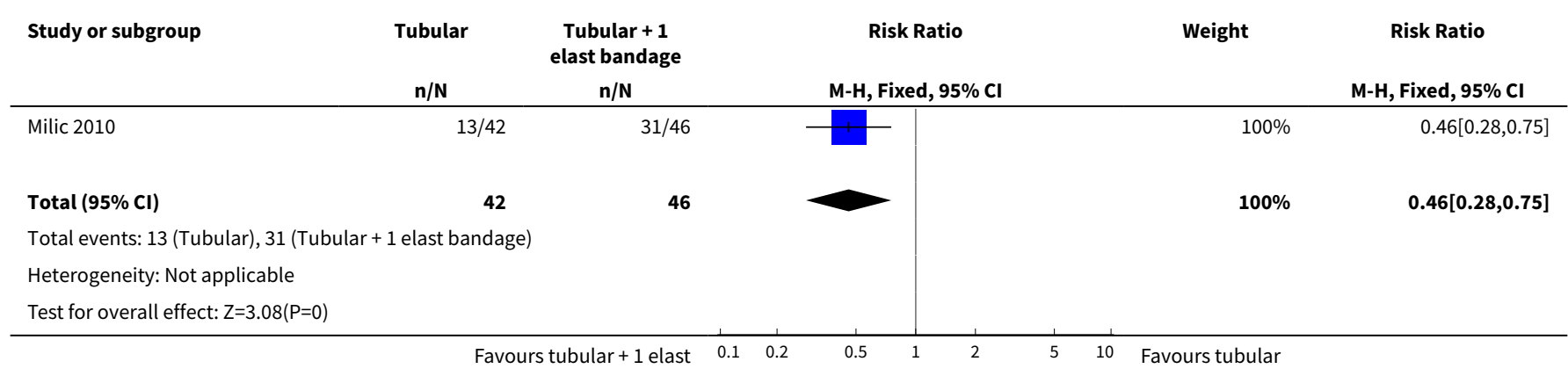

Comparison 22. Tubular compression vs tubular plus 2 elastic bandages

\begin{tabular}{llllll}
\hline Outcome or subgroup title & $\begin{array}{l}\text { No. of } \\
\text { studies }\end{array}$ & $\begin{array}{l}\text { No. of par- } \\
\text { ticipants }\end{array}$ & Statistical method & Effect size \\
\hline 1 Patients with complete healing at six months & 1 & 85 & Risk Ratio (M-H, Fixed, 95\% Cl) & $0.42[0.26,0.68]$ \\
\hline
\end{tabular}

Analysis 22.1. Comparison 22 Tubular compression vs tubular plus 2 elastic bandages, Outcome 1 Patients with complete healing at six months.

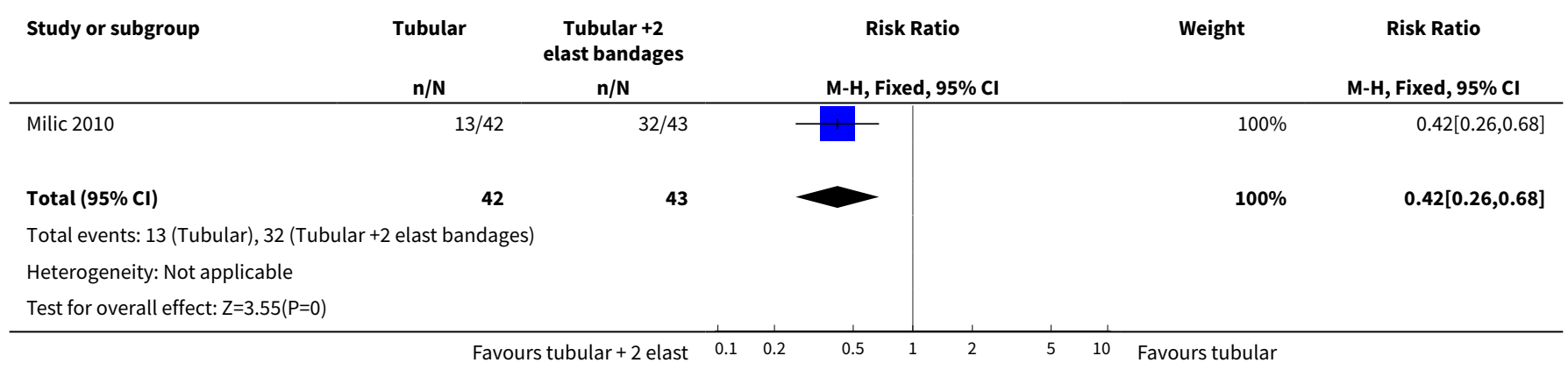

\section{Comparison 23. Tubular plus 1 elastic bandage vs tubular plus 2 elastic bandages}

\begin{tabular}{llll}
\hline Outcome or subgroup title & $\begin{array}{l}\text { No. of } \\
\text { studies }\end{array}$ & $\begin{array}{l}\text { No. of par- } \\
\text { ticipants }\end{array}$ & Statistical method \\
\hline 1 Patients with complete healing at six months & 1 & 89 & Risk Ratio (M-H, Fixed, 95\% Cl) \\
\hline
\end{tabular}


Analysis 23.1. Comparison 23 Tubular plus 1 elastic bandage vs tubular plus 2 elastic bandages, Outcome 1 Patients with complete healing at six months.

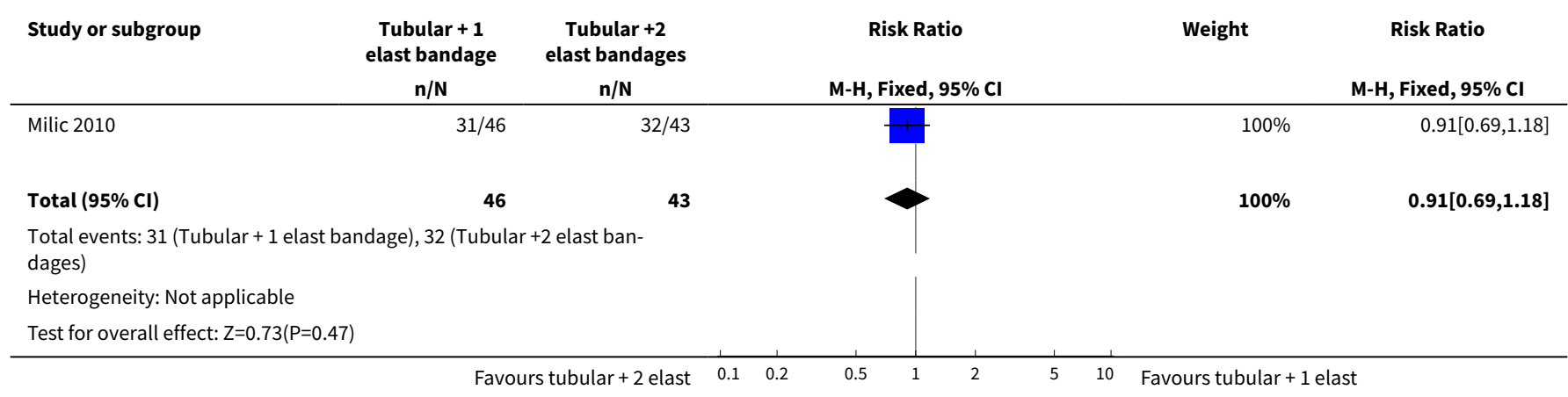

\section{ADDITIONAL TABLES}

Table 1. Summary of eligible trials for comparison of 4LB and SSB

\begin{tabular}{|c|c|c|c|c|c|c|}
\hline $\begin{array}{l}\text { Study (year of } \\
\text { main publica- } \\
\text { tion) } \\
\text { Country }\end{array}$ & $\begin{array}{l}\text { No. }(\%) \\
\text { of known } \\
\text { ran- } \\
\text { domised } \\
\text { patients }\end{array}$ & $\begin{array}{l}\text { No. of } \\
\text { study } \\
\text { centres }\end{array}$ & $\begin{array}{l}\text { Patient selection crite- } \\
\text { ria }\end{array}$ & $\begin{array}{l}\text { Median } \\
\text { follow-up } \\
\text { (weeks) for } \\
\text { non-healed } \\
\text { patients, de- } \\
\text { rived from } \\
\text { IPD }\end{array}$ & $\begin{array}{l}\text { No. patients ex- } \\
\text { cluded from trial- } \\
\text { ists' analyses vs } \\
\text { no. reinstated for } \\
\text { meta-analysis }\end{array}$ & $\begin{array}{l}\text { Reasons for } \\
\text { exclusion } \\
\text { from trialists' } \\
\text { analyses }\end{array}$ \\
\hline
\end{tabular}

\begin{tabular}{lllll}
\hline Unpublished trial $40(4.5 \%)$ & Unknown Unknown & NA & \\
UK
\end{tabular}

\begin{tabular}{lllll}
\hline Duby et al (1993) $43(4.8 \%)$ & 1 & $\begin{array}{l}\text { Venous leg ulcer; ABPI } \geq \quad N A \\
0.9 .\end{array}$
\end{tabular}

UK

\begin{tabular}{llllll}
\hline $\begin{array}{l}\text { Scriven et al } \\
(1998)\end{array}$ & $53(6.0 \%)$ & 1 & $\begin{array}{l}\text { Venous leg ulceration } \\
\text { confirmed with colour } \\
\text { duplex scanning and }\end{array}$ & 4LB 13.0 & 4LB: 1 vs 0 \\
UK & & SBPI $\geq 0.8$. & SSB: 2 vs 0
\end{tabular}

\begin{aligned} & low-up data, 1 died early in \\ & trial. \\ & toll \\ & \hline\end{aligned}

\begin{tabular}{|c|c|c|c|c|c|c|}
\hline $\begin{array}{l}\text { Partsch et al } \\
\text { (2001) } \\
\text { Austria/Nether- } \\
\text { lands }\end{array}$ & $\begin{array}{l}116 \\
(13.1 \%)\end{array}$ & 7 & $\begin{array}{l}\text { New episode of venous } \\
\text { leg ulceration; ulcer ae- } \\
\text { tiology confirmed by } \\
\text { Doppler or clinical histo- } \\
\text { ry; ABPI } \geq 0.8 \text {. }\end{array}$ & $\begin{array}{l}\text { 4LB } 7.0 \\
\text { SSB } 10.3\end{array}$ & Overall 4 vs $0^{\star}$ & $\begin{array}{l}3 \text { had no fol- } \\
\text { low-up data. } \\
1 \text { ineligible. }\end{array}$ \\
\hline $\begin{array}{l}\text { Ukat et al (2003) } \\
\text { Germany }\end{array}$ & $89(10.0 \%)$ & 2 & $\begin{array}{l}\text { Venous leg ulceration; } \\
A B P I \geq 0.8 \text {. }\end{array}$ & $\begin{array}{l}\text { 4LB } 11.9 \\
\text { SSB } 12.0\end{array}$ & 4LB: 0 vs 0 & \\
\hline
\end{tabular}


Table 1. Summary of eligible trials for comparison of 4LB and SSB (Continued)

\begin{tabular}{llllll} 
Franks et al (2004) & $\begin{array}{l}159 \\
(18.0 \%)\end{array}$ & 12 & $\begin{array}{l}\text { Venous leg ulceration; ul- } \\
\text { cer aetiology confirmed } \\
\text { by clinical history; ABPI } \geq \\
0.8 .\end{array}$ & SLB 23.7 & SLB: 1 vs 1 \\
UK & & & SSB: 2 vs 2 \\
\hline $\begin{array}{l}\text { Iglesias et al } \\
(2004)\end{array}$ & $\begin{array}{l}387 \\
(43.6 \%)\end{array}$ & 9 & $\begin{array}{l}\text { Venous leg ulcer } \geq 1 \mathrm{~cm} \\
\text { diameter; ABPI } \geq 0.8 .\end{array}$ & 4LB 55.0 & 4LB: 0 vs 0 \\
UK & & & SSB 54.0 & SSB: 0 vs 0
\end{tabular}

UK

$\begin{array}{llll}887 & 32 & \text { 4LB } 13.0 & \text { Overall } 10 \text { vs } 3 \\ (100 \%) & & \text { SSB } 12.3 & \end{array}$

\section{Abbreviations}

$4 \mathrm{LB}=$ four-layer bandage

$A B P I=$ ankle brachial pressure index

$I P D=$ individual patient data

$N A=$ not applicable as unable to retrieve individual patient data

SSB = short-stretch bandage - SSB

Note

* breakdown per group not provided.

Table 2. Characteristics of patients from trials with available IPD

\begin{tabular}{llcc}
\hline Variable & $\begin{array}{l}\text { Four-layer bandage }(\mathbf{n}= \\
\mathbf{3 9 4})\end{array}$ & $\begin{array}{l}\text { Short-stretch bandage }(\mathbf{n} \\
=\mathbf{4 0 3})\end{array}$ & Total (N=797) \\
\hline Sex & & & $317(39.8 \%)$ \\
Male & $151(38.3 \%)$ & $166(41.2 \%)$ & $479(60.1 \%)$ \\
Female & $242(61.4 \%)$ & $237(58.8 \%)$ & $1(0.1 \%)$ \\
Not recorded & $1(0.3 \%)$ & $0(0.0 \%)$ & \\
\hline
\end{tabular}

\section{Patient age (years)}

$\begin{array}{llll}\text { Mean (SD) } & 69.5(13.1) & 70.3(13.8) & 69.9(13.5) \\ \text { Median (min, max) } & 71.8(19,99) & 73.0(23,100) & 73.0(19,100) \\ \text { Not recorded } & 0(0.0 \%) & 1(0.3 \%) & 1(0.1 \%)\end{array}$

\section{Ulcer status}

$\begin{array}{lccc}\text { First } & 75(19.0 \%) & 86(21.3 \%) & 161(20.2 \%) \\ \text { Recurrent } & 287(72.8 \%) & 286(71.0 \%) & 573(71.9 \%) \\ \text { Not recorded } & 32(8.1 \%) & 31(7.7 \%) & 63(7.9 \%)\end{array}$

\section{Ulcer duration}

Up to 1.00 month

$120(30.5 \%)$

$122(30.3 \%)$

$242(30.4 \%)$

$1.01-6.00$ months

$157(39.8 \%)$

$173(42.9 \%)$

$330(41.4 \%)$ 
Table 2. Characteristics of patients from trials with available IPD

\begin{tabular}{|c|c|c|c|}
\hline $6.01-12.00$ months & $41(10.4 \%)$ & $40(9.9 \%)$ & $81(10.2 \%)$ \\
\hline Longer than 12 months & $73(18.5 \%)$ & $60(14.9 \%)$ & $133(16.7 \%)$ \\
\hline Not recorded & $3(0.8 \%)$ & $8(2.0 \%)$ & $11(1.4 \%)$ \\
\hline
\end{tabular}

\begin{tabular}{ll} 
Ulcer area $\left(\mathbf{c m}^{\mathbf{2}}\right)$ & \\
Mean (SD) & $13.7(36.7)$ \\
Median (min, max) & $4.3(0.2,378.3)$ \\
Not recorded & $21(5.3 \%)$ \\
\hline
\end{tabular}

\section{Presence of slough}

\begin{tabular}{|c|c|c|c|}
\hline Non-sloughy & $110(27.9 \%)$ & $128(31.8 \%)$ & $238(29.9 \%)$ \\
\hline Sloughy & 199 (50.5\%) & 177 (43.9\%) & $376(47.2 \%)$ \\
\hline Not recorded & 85 (21.6\%) & 98 (24.3\%) & $183(23.0 \%)$ \\
\hline
\end{tabular}

\section{Presence of granulation}

\begin{tabular}{|c|c|c|c|}
\hline Non-granulating & $122(31.0 \%)$ & $126(31.3 \%)$ & $248(31.1 \%)$ \\
\hline Granulating & $187(47.4 \%)$ & $184(45.7 \%)$ & $371(46.6 \%)$ \\
\hline Not recorded & 85 (21.6\%) & $93(23.1 \%)$ & $178(22.3 \%)$ \\
\hline
\end{tabular}

\section{Presence of epithelialising tissue}

$\begin{array}{lccc}\text { Non-epithelialising } & 226(57.4 \%) & 221(54.8 \%) & 447(56.1 \%) \\ \text { Epithelialising } & 29(7.4 \%) & 32(7.9 \%) & 61(7.7 \%) \\ \text { Not recorded } & 139(35.3 \%) & 150(37.2 \%) & 289(36.3 \%)\end{array}$

\section{Ankle-brachial pressure index}

$\begin{array}{llll}\text { Mean (SD) } & 1.09(0.18) & 1.08(0.15) & 1.08(0.16) \\ \text { Median (min, max) } & 1.05(0.76,2.00) & 1.06(0.75,1.70) & 1.06(0.75,2.00) \\ \text { Not recorded } & 19(4.8 \%) & 11(2.7 \%) & 30(3.8 \%)\end{array}$

\section{Ankle circumference}

\begin{tabular}{llll} 
Mean (SD) & $23.9(2.8)$ & $23.9(2.8)$ & $23.9(2.8)$ \\
Median (min, max) & $24.0(16.2,34.0)$ & $24.0(16.0,33.0)$ & $24.0(16.0,34.0)$ \\
Not recorded & $3(0.8 \%)$ & $6(1.5 \%)$ & $9(1.1 \%)$ \\
\hline Ankle mobility & & $294(73.0 \%)$ & $583(73.1 \%)$ \\
Fully mobile & $289(73.4 \%)$ & $104(25.8 \%)$ & $207(26.0 \%)$ \\
Impaired & $103(26.1 \%)$ & $5(1.2 \%)$ & $7(0.9 \%)$ \\
Not recorded & $2(0.5 \%)$ & &
\end{tabular}

\section{Patient mobility}


Table 2. Characteristics of patients from trials with available IPD (Continued)

\begin{tabular}{|c|c|c|c|}
\hline Impaired & $103(26.1 \%)$ & $111(27.6 \%)$ & 214 (26.9\%) \\
\hline Not recorded & 27 (6.9\%) & $27 \quad(6.7 \%)$ & $54(6.8 \%)$ \\
\hline \multicolumn{4}{|c|}{ History of DVT } \\
\hline No DVT & $147(37.3 \%)$ & $165(40.9 \%)$ & $312(39.1 \%)$ \\
\hline DVT & $52(13.2 \%)$ & $46(11.4 \%)$ & $98(12.3 \%)$ \\
\hline Not recorded & $195(49.5 \%)$ & $192(47.6 \%)$ & 387 (48.6\%) \\
\hline
\end{tabular}

\section{Abbreviations}

DVT = deep vein thrombosis

$\max =$ maximum value in range

$\min =$ minimum value in range

$\mathrm{SD}=$ standard deviation.

\section{Note}

Figures are numbers (percentages) of patients unless stated otherwise.

Table 3. Final model based on five trials

\begin{tabular}{|c|c|c|c|c|c|}
\hline Variable & $\boldsymbol{\beta}$ & SE $(\beta)$ & $H R$ & 95\% Cl for $H R$ & $P$ value \\
\hline Bandage & 0.27 & 0.10 & 1.31 & 1.09 to 1.58 & $=0.005$ \\
\hline Duration overall & & & & & $<0.001$ \\
\hline $\begin{array}{l}\text { Duration category } 1.01-6.0 \text { months versus } 0-1 \\
\text { month }\end{array}$ & -0.12 & 0.11 & 0.89 & 0.71 to 1.11 & $=0.293$ \\
\hline $\begin{array}{l}\text { Duration category } 6.01-12.0 \text { months versus } 0-1 \\
\text { month }\end{array}$ & -0.53 & 0.19 & 0.59 & 0.40 to 0.85 & $=0.005$ \\
\hline Duration category $>12$ months versus $0-1$ month & -1.07 & 0.19 & 0.35 & 0.24 to 0.50 & $<0.001$ \\
\hline $\log _{e}$ ulcer area & -0.36 & 0.05 & 0.70 & 0.64 to 0.77 & $<0.001$ \\
\hline
\end{tabular}

\section{Abbreviations}

$\beta=$ regression coefficient

$\mathrm{Cl}=$ confidence interval

$\mathrm{HR}=$ hazard ratio

$\log _{\mathrm{e}}=$ natural logarithm

$\mathrm{SE}=$ standard error of regression coefficient

Table 4. Final model based on four trials

\begin{tabular}{llllll}
\hline Variable & $\boldsymbol{\beta}$ & $\boldsymbol{S E}(\boldsymbol{\beta})$ & $\boldsymbol{H R}$ & 95\% CI for HR & $\boldsymbol{P}$ value \\
\hline Bandage & 0.25 & 0.10 & 1.29 & 1.06 to 1.57 & $=0.011$ \\
\hline $\begin{array}{l}\text { Duration overall } \\
\begin{array}{l}\text { Duration category 1.01-6.0 months versus 0-1 } \\
\text { month }\end{array}\end{array}$ & -0.12 & 0.12 & 0.88 & 0.71 to 1.11 & $<0.001$ \\
\hline
\end{tabular}


Table 4. Final model based on four trials (Continued)

\begin{tabular}{llllll}
$\begin{array}{l}\text { Duration category 6.01-12.0 months versus 0-1 } \\
\text { month }\end{array}$ & -0.51 & 0.20 & 0.60 & 0.41 to 0.90 & $=0.013$ \\
\hline Duration category > 12 months versus 0-1 month & -1.17 & 0.21 & 0.31 & 0.21 to 0.47 & $<0.001$ \\
\hline $\begin{array}{l}\text { Loge ulcer area } \\
\text { Recurrent ulceration }\end{array}$ & -0.35 & 0.05 & 0.70 & 0.64 to 0.78 & $<0.001$ \\
\hline
\end{tabular}

\author{
Abbreviations \\ $\beta=$ regression coefficient \\ $\mathrm{Cl}=$ confidence interval \\ $\mathrm{HR}=$ hazard ratio \\ $\log _{e}=$ natural logarithm \\ $\mathrm{SE}=$ standard error of regression coefficient
}

\title{
AP PEN DICES
}

\section{Appendix 1. Search strategy - Original version}

The search strategy of the Cochrane Wounds Group was used to identify RCTs and CCTs of bandages or stockings in the treatment of venous leg ulcer trials (see Scope of Wounds Group). This includes electronic searches of MEDLINE, CINAHL, EMBASE, The Cochrane Controlled Trials Register, as well as hand searches of conference proceedings and wound care journals.

Experts in wound care and pharmaceutical companies were contacted to enquire about unpublished, ongoing and recently published trials.

Citations within obtained reviews and papers were scrutinised to identify additional studies.An Advisory Panel was established at the outset of a series of reviews of which this is one. They assisted by checking our lists of trials for any omissions, and to inform us of any unpublished, ongoing or recently completed trials.

\section{Appendix 2. Ovid MEDLINE search strategy}

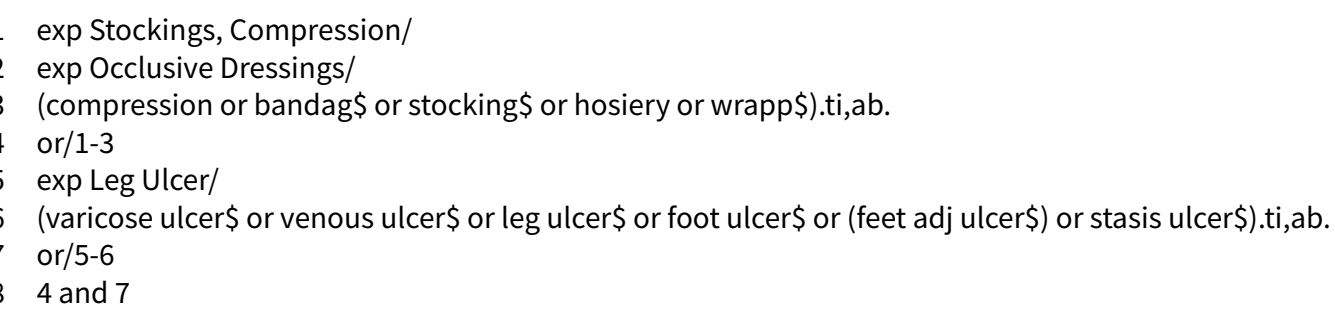

\section{Appendix 3. Ovid EMBASE search strategy}

1 exp Compression Therapy/

(compression or bandag $\$$ or stocking $\$$ or hosiery or wrapp $\$$ ).ti,ab.

or/ $1-2$

exp Leg Ulcer/

(varicose ulcer\$ or venous ulcer\$ or leg ulcer\$ or foot ulcer\$ or (feet adj ulcer\$) or stasis ulcer\$).ti,ab.

or/4-5

73 and 6

\section{Appendix 4. Ovid CINAHL search strategy}

1 exp Bandaging Techniques/

2 exp Compression Therapy/

3 (compression or bandag\$ or stocking $\$$ or hosiery or wrapp\$).ti,ab.

4 or/1-3

5 exp Leg Ulcer/

6 (varicose ulcer\$ or venous ulcer\$ or leg ulcer\$ or foot ulcer\$ or (feet adj ulcer\$) or stasis ulcer\$).ti,ab.

7 or/5-6 
84 and 7

WHAT'S NEW

\begin{tabular}{lll}
\hline Date & Event & Description \\
\hline 20 February 2013 & Amended & Background text updated \\
\hline
\end{tabular}

\section{HIST ORY}

Protocol first published: Issue 1, 1998

Review first published: Issue 2, 1998

\begin{tabular}{lll}
\hline Date & Event & Description \\
\hline 23 October 2012 & Amended & Amendment to NIHR acknowledgement statement \\
\hline 20 September 2012 & New search has been performed & Second update, new search. \\
\hline 20 September 2012 & $\begin{array}{l}\text { New citation required and conclusions } \\
\text { have changed }\end{array}$ & $\begin{array}{l}\text { Nine new trials included, IPD meta analysis, conclusions updat- } \\
\text { ed. }\end{array}$ \\
\hline 14 October 2008 & $\begin{array}{l}\text { New citation required and conclusions } \\
\text { have changed }\end{array}$ & First update, new trials added, conclusions changed. \\
\hline 25 April 2008 & Amended & Converted to new review format. \\
\hline 25 February 2004 & Amended & minor update \\
\hline 5 February 2001 & $\begin{array}{l}\text { New citation required and conclusions } \\
\text { have changed }\end{array}$ & Substantive amendment \\
\hline
\end{tabular}

\section{CONTRIBUTIONS OF AUTHORS}

Susan O'Meara: co-ordinated both review updates, undertook study selection, extracted data and undertook quality assessment. Wrote to study authors, experts, and companies. Analysed and interpreted data, performed statistical analysis and completed the first draft of both review updates. Approved both final review updates prior to submission. Made an intellectual contribution to the review updates and is guarantor of the review.

Nicky Cullum: undertook study selection, checked quality of data extraction and undertook quality assessment. Analysed or interpreted data and checked quality assessment and statistical analysis. Interpreted data and performed part of writing or editing of the original review and the updated review. Made an intellectual contribution to the review and the update. Advised on the review and the update and approved both the review and the review update prior to submission. Secured funding for updating the review and performed previous work that was the foundation of the current review.

Andrea Nelson: for the original review: extracted data, undertook quality assessment and checked quality of data extraction and quality assessment. Analysed or interpreted data and provided data. Performed part of writing or editing the review, made an intellectual contribution to the review and approved the original review prior to submission. For the first update of this review: provided data and performed previous work that was the foundation of the current review update. Performed part of writing or editing of the review update, made an intellectual contribution to the review update and approved the final review update prior to submission. For the second update of the review, commented on the review draft and approved the final review update prior to submission

Jo Dumville: undertook study selection, checked quality of data extraction and quality assessment, provided comments on the draft. 


\section{Contributions of editorial base:}

Sally Bell-Syer: co-ordinated the editorial process; advised on interpretation and content; edited the updated review. Ruth Foxlee: designed the search strategy, edited the search methods section for the update and ran the searches.

\section{DECLARATIONS OF INTEREST}

Nicky Cullum, Susan O'Meara and Jo Dumville receive funding from the National Institute for Health Research (NIHR) under its Programme Grants for Applied Research funding scheme. This study presents independent research funded by the National Institute for Health Research (NIHR) under its Programme Grants for Applied Research funding scheme (RP-PG-0407-10428). The views expressed in this review are those of the author(s) and not necessarily those of the NHS, the NIHR or the Department of Health.

Nicky Cullum is an NIHR Senior Investigator.

Nicky Cullum was Principal Investigator for one of the trials included in this review (Iglesias 2004); this trial was commissioned after the first version of this review was completed. Nicky Cullum is currently Co-Investigator on the UK NIHR HTA Programme VenUS IV trial which will be eligible for a future update of this review.

Andrea Nelson was co-applicant and trial co-ordinator for one of the trials included in this review (Iglesias 2004); this trial was commissioned after the first version of this review was completed. Andrea Nelson was trial co-ordinator and author of the main publication for one of the trials included in this review (Nelson 2007a).

\section{SOURCES OF SUPPORT}

\section{Internal sources}

- Department of Health Sciences, University of York, UK.

- School of Healthcare, University of Leeds, UK.

Time and IT resources for EA Nelson to contribute to update of review

\section{External sources}

- NIHR Programme Grants for Applied Research, UK.

- NIHR Fellowship Scheme, UK.

A Researcher Development Award in Evidence Synthesis to Susan O'Meara

- NIHR/Department of Health (England), Cochrane Wounds Group, UK.

\section{DIFFERENCES BETWEEN PROTOCOL AND REVIEW}

\section{Note: differences between this review and the previous versions:}

1. Controlled clinical trials (CCTs) were eligible for inclusion in the original version of the review. Updated versions of the review have restricted inclusion to studies describing treatment allocation as random. In consequence, two studies have been excluded from this review that were previously included. The comparisons involved were: compression (Unna's Boot) versus no compression (dressing alone) (Sikes 1985), and compression stockings versus SSB (Horakova 1994).

2. A third trial that evaluated two different three-component systems was excluded from updated versions of the review because of confounding of the treatment effect by administration of steroids in one treatment arm (Northeast 1990).

3. Previously, the trial by Eriksson 1986 was entered as a secondary reference for Eriksson 1984. Further scrutiny revealed that these are two separate evaluations. In the current review, the two trials have been included and reported separately.

4. Previously the trial by Rubin 1990 was described as comparing compression with primary dressing alone. Further study of the report suggested that the comparison group received a primary dressing plus elastic bandage used as a retaining wrap. Comments in the trial authors' discussion section suggest that these bandages did not provide compression. Therefore, this trial has now been reviewed in a section comparing compression with non-compressive bandages

\section{N DEX TERMS}

\section{Medical Subject Headings (MeSH)}

${ }^{\star}$ Bandages [economics]; *Stockings, Compression [economics]; Cost-Benefit Analysis; Randomized Controlled Trials as Topic; Varicose Ulcer [*therapy]; Wound Healing

\section{MeSH check words}

Humans 